\title{
Experimental Investigation of Coherent Structures Generated by Active and Passive Separation Control in Turbulent Backward-Facing Step Flow
}

\author{
Dissertation \\ for the award of the degree \\ "Doctor rerum naturalium" \\ of the Georg-August-Universität Göttingen \\ within the doctoral program PROPHYS \\ of the Georg-August University School of Science (GAUSS) \\ submitted by \\ Xingyu Ma \\ from Hebei, China
}

Göttingen, 2015 


\section{$\underline{\text { Thesis Committee }}$}

\section{Prof. Dr. Andreas Dillmann}

Institute of Aerodynamics and Flow Technology, German Aerospace Center

\section{Prof. Dr. Martin Rein}

Institute of Aerodynamics and Flow Technology, German Aerospace Center

\section{Dr. Andreas Schröder}

Institute of Aerodynamics and Flow Technology, German Aerospace Center

\section{Members of the Examination Board}

\section{Reviewer: Prof. Dr. Andreas Dillmann}

Institute of Aerodynamics and Flow Technology, German Aerospace Center

Second Reviewer: Prof. Dr. Martin Rein

Institute of Aerodynamics and Flow Technology, German Aerospace Center

\section{Further members of the Examination Board}

\section{Prof. Dr. Eberhard Bodenschatz}

Department of Fluid Dynamics, Pattern Formation and Nanobiocomplexity, Max Planck Institute for Dynamics and Self-Organization

Prof. Dr. Andreas Tilgner

Institute of Geophysics, Georg-August-University of Göttingen

\section{Prof. Dr. Wolfgang Glatzel}

Institute of Astrophysics, Georg-August-University of Göttingen

\section{Prof. Dr. Markus Raffel}

Institute of Turbomachinary and Fluid Dynamics, Leibniz-University of Hannover

Date of the oral examination: 21. 07. 2015 


\section{Abstract}

This dissertation presents the experimental investigation of coherent structures which were generated by active and passive separation control devices in a turbulent backward-facing step (BFS) flow. The Reynolds number was $\operatorname{Re}_{\mathrm{h}}=2.0 \times 10^{4}$, based on the free-stream velocity and the backward-facing step height. Three types of flow control devices, which are referred to as acoustic tube (AT), oscillating flap (OF) and vortex generators (VGs), were implemented independently on the backward-facing step in order to control the turbulent flow separation downstream of the step. For each implementation of the devices, 2D-2C planar particle image velocimetry was used to measure the separated shear layer, recirculation region and reattachment area downstream. Moreover, 3D-3C(t) time-resolved tomographic particle image velocimetry was employed, within a three-dimensional volume at a high sampling frequency, to measure the three-dimensional fluid motions in the separated shear layer downstream of the vortex generators.

The first flow control device under investigation in the present work was the acoustic tube. As an active flow control device, the acoustic tube generated periodic pressure perturbations influencing the flow through a spanwise thin slot close to the separation edge at an actuation frequency of $f_{a}=100 \mathrm{~Hz}$, which was close to the most-amplified frequency of the shedding instability of the turbulent shear layer. The flow control results show that the acoustic tube can suppress the recirculation region and reduce the reattachment length by $43.7 \%$. The rolling-up and pairing processes of spanwise vortices lead to an increase of the Reynolds shear stress. The coherent structures are extracted by proper orthogonal decomposition (POD) and are represented by two pairs of the spatial modes. Both the primary and secondary series of vortices 
are reconstructed as travelling waves corresponding to the actuation frequency $f_{a}$ and the overtone frequency $2 \cdot f_{a}$, respectively.

The second flow control device under investigation was the oscillating flap. The oscillating flap was driven by periodic Ampere force and thereby generated small perturbations over the separation edge at a perturbation frequency of $\mathrm{f}_{\mathrm{p}}=55 \mathrm{~Hz}$, which was close to the half of the most-amplified frequency of the shedding instability of the turbulent shear layer. The time-averaged velocity fields show that the small perturbations can reduce the recirculation region behind the step and reduce the reattachment length by $31.0 \%$. The phase-averaged results indicate a small streamwise ejection with a velocity of $0.7 \cdot \mathrm{U}_{0}$ when the oscillating flap moves downwards. The rolling-up and breakdown processes of the spanwise vortices result in considerable increases of coherent and incoherent parts of the Reynolds shear stress. The coherent structures in the turbulent shear layer are further analyzed by snapshot proper orthogonal decomposition (POD) and spatial dynamic mode decomposition (DMD) in energy and dynamic approaches complementarily. It is shown that the periodic small perturbations enable to manipulate the large-scale separated shear layer in a flapping motion and therefore effectively reduce the reattachment length downstream of the step.

The third flow control device was an array of passive vortex generators. Low-profile wedge-type vortex generators (VGs) were fixed on the surface upstream of the step in the spanwise direction, which were totally submerged within the turbulent boundary layer. The time-averaged reattachment length is reduced by $29.1 \%$ due to the VGs. Two-point cross-correlation functions were used in order to analyze the spatial and temporal evolution of the VG-induced vortices. The Reynolds shear stress is considerably increased, which is, moreover, highly phase-correlated with the VG-induced coherent structures. POD and DMD are applied to analyze the complex vortex structures in the spatial and temporal approaches, respectively. First, two series of quasi-periodic oblique vortices in the streamwise-spanwise orientation are extracted by the POD method, which contain a highly coherent bandwidth of 
Strouhal number $0.3<\mathrm{St}_{\mathrm{h}}<0.6$. Second, by applying the DMD to the POD reconstructed flow field, a single-frequency dynamic mode at $\mathrm{St}_{\mathrm{h}}=0.37$ is extracted and $\Lambda$-shaped vortex structures are reconstructed in the recovery of the predominant DMD mode.

Experimental results show that the three types of flow control devices are able to reduce the reattachment length by generating quasi-periodic coherent motions in the separated shear layer. These coherent structures lead to an increase of Reynolds shear stress and play an important role in the momentum transfer in the turbulent shear flow. 


\section{Contents}

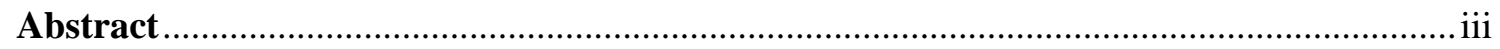

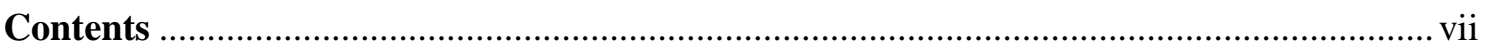

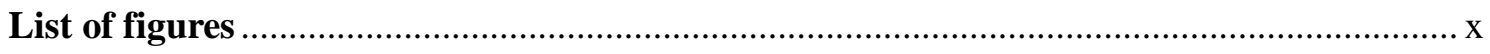



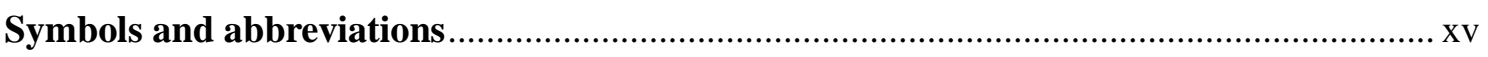

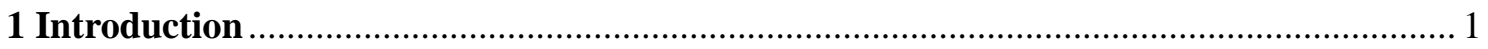

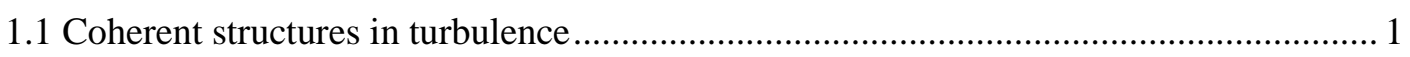

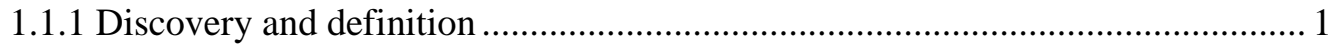

1.1.2 Approaches to coherent structures................................................................. 2

1.1.3 Coherent structures in turbulent shear flows ................................................... 4

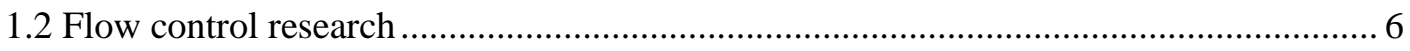



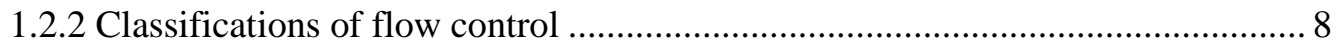

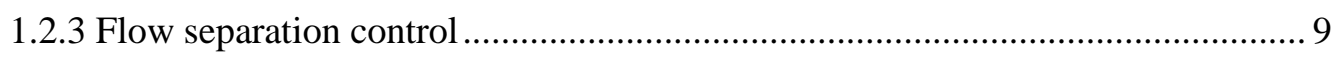

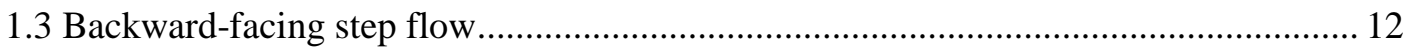

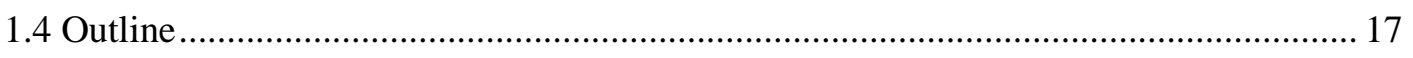





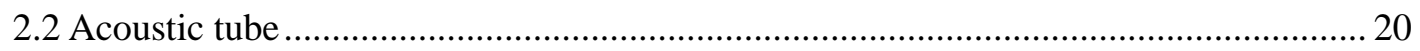

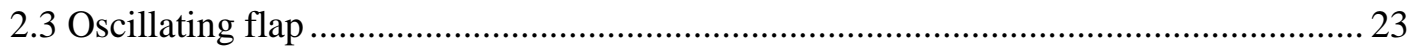

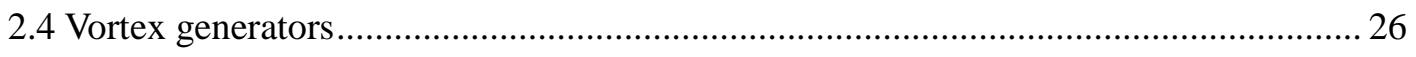



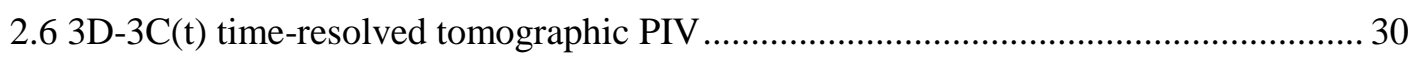




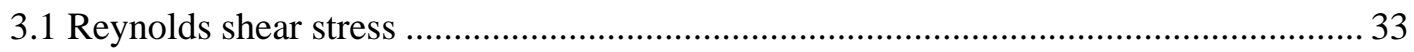



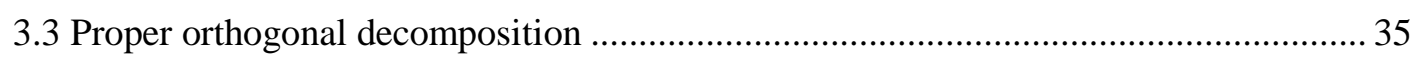

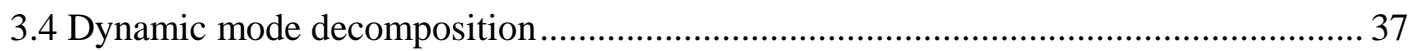

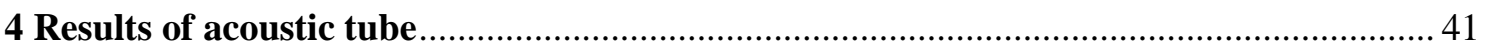

4.1 Convergence of mean and root-mean-square velocities .......................................... 42

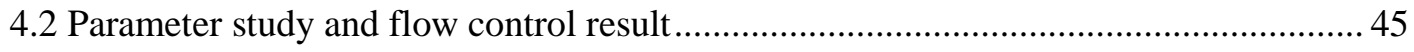

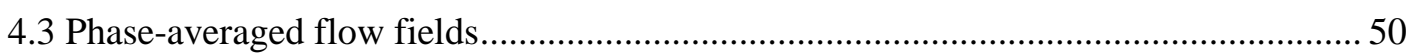

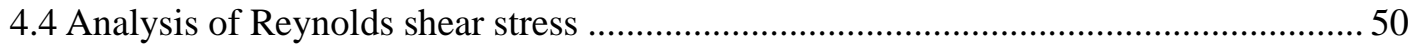

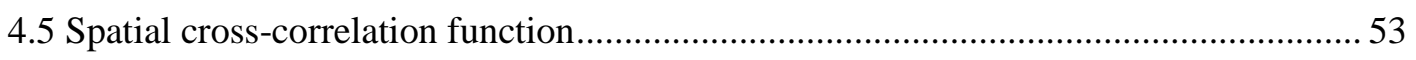





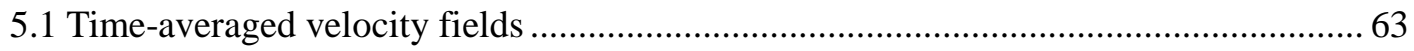



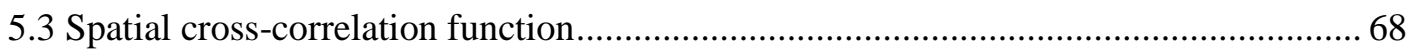

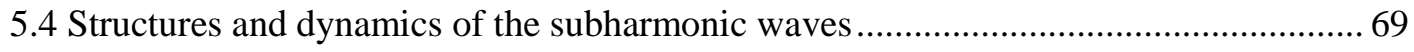

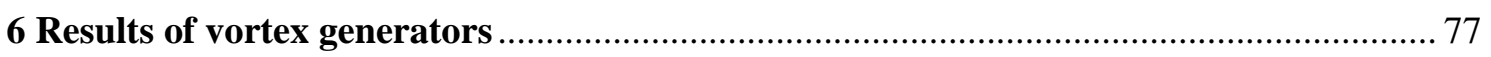

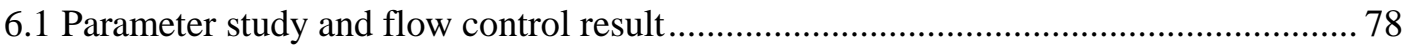

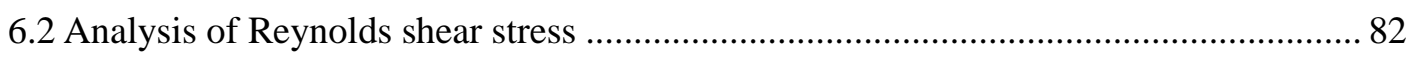

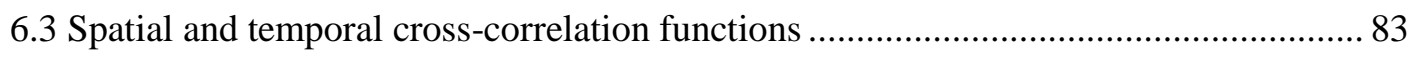



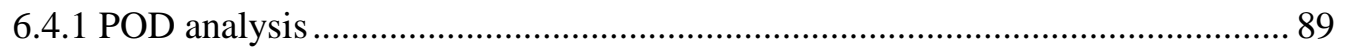

6.4.2 DMD analysis based on POD reconstruction ................................................... 96

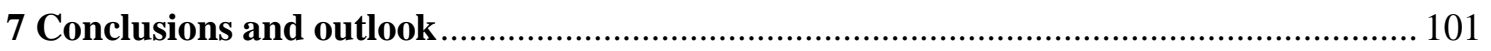

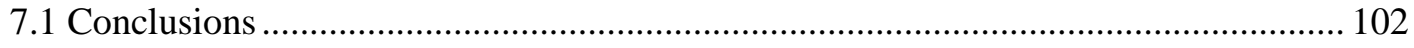

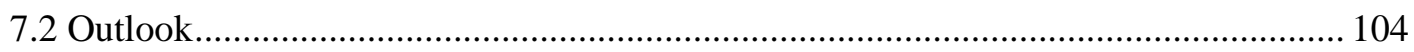

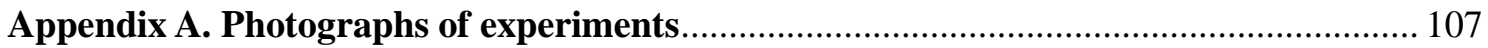


Appendix B. Publications



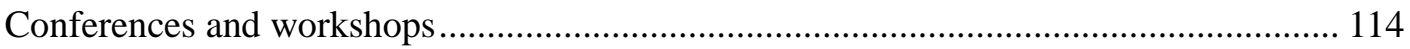

Lectures and teaching assistance in the University of Göttingen ..................................... 115

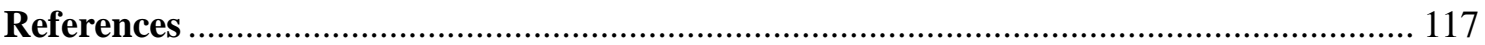

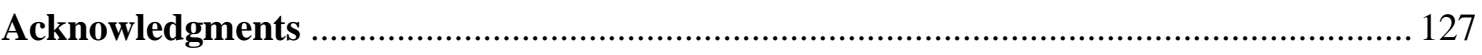

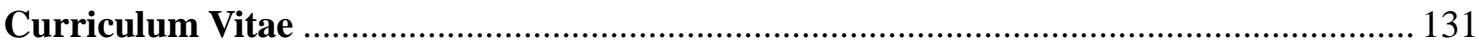




\section{List of figures}

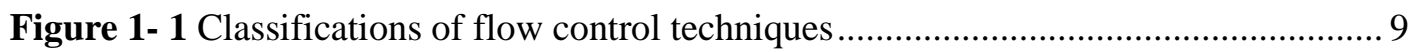

Figure 1- 2 Aerodynamic characteristics of a two-dimensional NACA 0012 airfoil. ........ 10

Figure 1- 3 Schematic diagram of turbulent backward-facing step flow .......................... 13

Figure 2- 1 1m-low-speed wind tunnel at the German Aerospace Center in Göttingen..... 20

Figure 2- 2 Photograph of the acoustic tube implemented with the backward-facing step 21

Figure 2- 3 Schematic diagram of acoustic tube integrated with backward-facing step .... 21

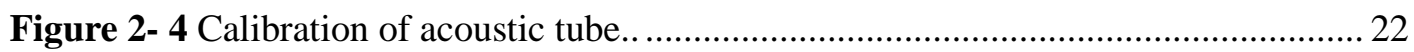

Figure 2- 5 Frequency spectrum of acoustic actuation at $\mathrm{f}_{\mathrm{a}}=100 \mathrm{~Hz}$ and $\mathrm{SPL}=110 \mathrm{~dB} .23$

Figure 2- 6 Photograph of the oscillating flap on the backward-facing step..................... 24

Figure 2- 7 Schematic diagram of oscillating flap in backward-facing step flow .............. 25

Figure 2- 8 Frequency spectrum of the oscillating flap at $\mathrm{f}_{\mathrm{p}}=55 \mathrm{~Hz}$............................ 26

Figure 2- 9 Photograph of the "VG-H10b" configuration on the BFS .............................. 27

Figure 2- 10 Schematic diagram of the BFS geometry and "VG-H10b" configuration..... 27

Figure 2- 11 Schematic diagram of 2D-2C PIV system and the acoustic tube................... 29

Figure 2- 12 Schematic diagram of 2D-2C PIV system and the oscillating flap................ 29

Figure 2- 13 Schematic diagram of 2D-2C PIV system and the vortex generators............ 30

Figure 2- 14 Schematic diagram of tomographic PIV system and the vortex generators .. 31

Figure 4- 1 Instantaneous velocity vector fields of the backward-facing step flow.. ......... 42

Figure 4- 2 Velocity contours of the clean case of the BFS flow ....................................... 44

Figure 4- 3 Sketch of the five discrete points and field of view ....................................... 44

Figure 4- 4 Convergence of mean and r.m.s. velocities at five discrete points .................. 45

Figure 4- 5 Convergence history of the mean and r.m.s. velocities in the field of view .... 45 
Figure 4- 6 Phase-averaged streamlines at the same $\mathrm{SPL}=110 \mathrm{~dB}$

Figure 4- 7 Phase-averaged streamlines at the same frequency $\mathrm{St}_{\mathrm{h}}=0.3 \ldots \ldots \ldots \ldots \ldots \ldots \ldots \ldots . . .47$

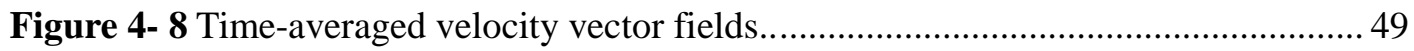

Figure 4- 9 Time-averaged velocity vector fields of the clean and the controlled cases .... 49

Figure 4- 10 Phase-averaged flow vector fields of the controlled case..............................50

Figure 4- 11 Total Reynolds shear stress (a) clean case; (b) controlled case ..................... 51

Figure 4- 12 Decomposition of Reynolds shear stress of the controlled case .................... 52

Figure 4- 13 Phase-averaged incoherent Reynolds shear stress of the controlled case ...... 53

Figure 4- 14 Contours of coefficient of spatial cross-correlation.....................................54

Figure 4- 15 Sketch of the rectangular region of $1.7 \mathrm{~h} \times 1 \mathrm{~h}$ in which POD is applied ......... 55

Figure 4- 16 POD eigenvalue distributions of the first 100 modes .................................... 56

Figure 4- 17 Scatter distributions of POD coefficients. .................................................. 57

Figure 4- 18 Reconstructed by $\mathrm{POD}_{1}+\mathrm{i} \cdot \mathrm{POD}_{2}$ of the controlled case............................... 59

Figure 4- 19 Reconstructed by $\mathrm{POD}_{5}+\mathrm{i} \cdot \mathrm{POD}_{6}$ of the controlled case............................... 60

Figure 4- 20 Phase angle relation between primary and secondary vortices...................... 61

Figure 5- 1 Time-averaged velocity vector and contour fields..........................................6 63

Figure 5- 2 Total Reynolds shear stress (a) clean case; (b) controlled case ....................... 64

Figure 5- 3 Decomposition of Reynolds shear stress of the controlled case...................... 65

Figure 5- 4 Phase-averaged incoherent Reynolds shear stress of the controlled case........ 66

Figure 5- 5 Phase-averaged contours of streamwise velocity $\quad$ (a) $\alpha=60^{\circ}$; (b) $\alpha=240^{\circ} .66$

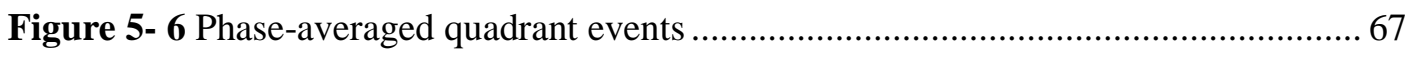

Figure 5- 7 Contours of coefficient of spatial cross-correlation........................................ 69

Figure 5- 8 Sketch of the rectangular region of $3.3 \mathrm{~h} \times 1.3 \mathrm{~h}$ in which POD is applied ........ 70

Figure 5- 9 (a) POD eigenvalues; (b) Scatter distributions of POD coefficients ................ 71

Figure 5- 10 POD Reconstruction by $\mathrm{POD}_{1}$ and $\mathrm{POD}_{2}$ of the controlled case.................. 71

Figure 5- 11 (a) Spatial DMD amplitude; (b) DMD growth rate in frequency doman...... 72




Figure 5- 13 Spatial developments of vertical velocity components $v$ at $\mathrm{Y} / \mathrm{h}=1$

Figure 6- 1 Streamwise mean velocity profiles in the turbulent shear layer ..................... 78

Figure 6- 2 Time-averaged velocity profile and fluctuations of boundary layer................ 79

Figure 6- 3 Time-averaged velocity vector fields of the four VG configurations. .............. 80

Figure 6- 4 Comparison of time-averaged velocity vector fields...................................... 80

Figure 6- 5 time-averaged velocity vector field of the clean case.................................... 81

Figure 6- 6 Comparison of Reynolds shear stress. (a) clean case; (b) controlled case.. .... 82

Figure 6- 7 Reynolds shear stress of the four VG configurations. ................................... 83

Figure 6- 8 Iso-surfaces of coefficient of spatial cross-correlation.................................... 85

Figure 6- 9 Iso-surfaces of coefficient of temporal cross-correlation. ............................... 86

Figure 6- 10 Iso-surfaces of coefficient of temporal cross-correlation ............................. 87

Figure 6- 11 Iso-surfaces of coefficient of temporal cross-correlation............................... 88

Figure 6- 12 POD eigenvalue distributions of the first 100 modes .................................. 89

Figure 6- 13 Temporal evolutions of the coefficients of $\mathrm{POD}_{1}$ and $\mathrm{POD}_{2} \ldots \ldots \ldots \ldots \ldots \ldots \ldots . . . . . . . . . . .91$

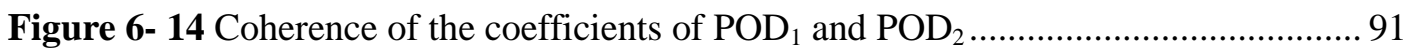

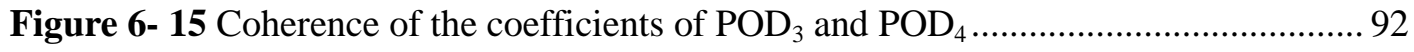

Figure 6- 16 Temporal evolutions of reconstruction by $\mathrm{POD}_{1}$ and $\mathrm{POD}_{2} \ldots \ldots \ldots \ldots \ldots \ldots \ldots \ldots . . . . . . . . . . . .94$

Figure 6- 17 Temporal evolutions of reconstruction by $\mathrm{POD}_{3}$ and $\mathrm{POD}_{4 . \ldots \ldots \ldots \ldots \ldots \ldots \ldots \ldots . . . . . . . . . . . . .95}$

Figure 6- 18 Phase-averaged Reynolds shear stress and POD reconstructed vortices ....... 96

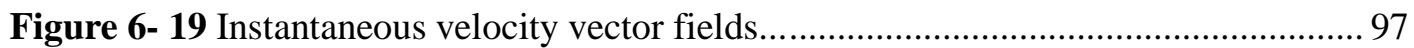

Figure 6- 20 DMD amplitude distribution in the frequency domain................................ 98

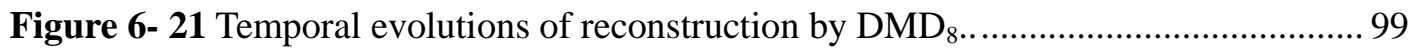

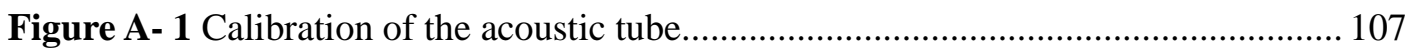

Figure A- 2 Preliminary test of the oscillating flap before wind tunnel test..................... 108

Figure A- 3 Acoustic measurement of the sound pressure level near the oscillating flap 108

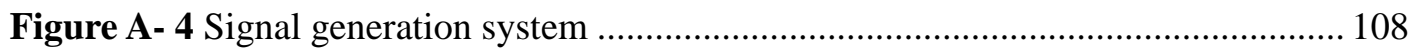


Figure A- 5 Vortex generators $(\mathrm{H}=10 \mathrm{~mm}$, forward-orientated $)$ 109



Figure A- 7 Zigzag bands near the leading edge of the flat plate ................................... 109

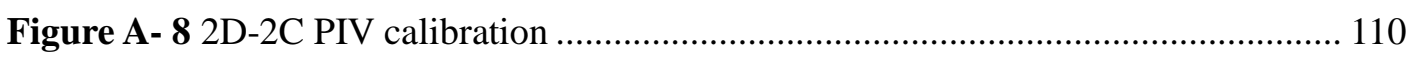

Figure A- 9 PCO.4000 high-resolution camera with the Nikon lens ............................... 110

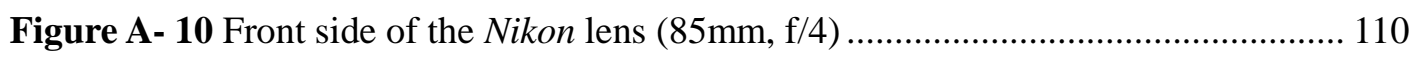

Figure A- 11 Ultra CFR Nd:YAG laser system from Big Sky inc....................................111

Figure A- 12 Plane mirror mounted downstream of the BFS .........................................111

Figure A- 13 Laser light sheet in the 2D-2C PIV measurement of the acoustic tube........111

Figure A- 14 Laser light sheet in the 2D-2C PIV measurement of the oscillating flap.... 112

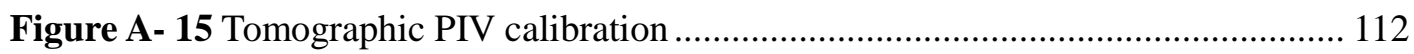

Figure A- 16 Four Photron APX-RS high-speed cameras with Nikon lenses .................. 112

Figure A- 17 High-repetition-rate laser generator from LEE inc. .................................. 113 


\section{List of tables}

Tabel 1- 1 Comparison of the present BFS flow and the literature …............................... 16

Tabel 1- 2 Flow control results of present study and the literature .................................... 16

Tabel 1-3 Hierarchy of spatial scales of the present BFS flow ........................................ 16

Table 2- 1 Parameters of 2D-2C and tomographic PIV systems ....................................... 32

Table 4- 1 Reduction of reattachment length of different parameter sets .......................... 47

Table 4- 2 Reduction of reattachment length in the present experiment and the literature 48

Table 6- 1 Reduction of reattachment length of different VG configurations .79 


\section{Symbols and abbreviations}

A

$A, A^{-1}$

$a_{i}$

$\mathrm{amp}_{\mathrm{i}}$

c

$\mathrm{C}_{\mathrm{ij}}$

$\mathrm{dt}$

$\mathrm{DMD}_{\mathrm{j}}, \boldsymbol{\psi}_{\mathbf{j}}$

e

e

$\mathbf{F}$

$\mathrm{f}_{0}$

$f_{a}$

$\mathrm{f}_{\mathrm{i}}$

$f_{p}$

$\mathrm{h}$

$\mathrm{H}$

1

$\mathrm{L}$

n

$\mathbf{P}$

$P_{i j}$

$\mathrm{POD}_{\mathrm{i}}, \boldsymbol{\phi}_{\mathrm{i}}$

$\mathrm{Q}_{1}, \mathrm{Q}_{2}, \mathrm{Q}_{3}, \mathrm{Q}_{4}$ peak amplitude of the oscillating flap [mm]

DMD amplitude matrix and its inverse matrix

POD coefficient

DMD amplitude of the i-th mode

speed of sound, $343[\mathrm{~m} / \mathrm{s}]$

coherence between two POD coefficient $\mathrm{a}_{\mathrm{i}}$ and $\mathrm{a}_{\mathrm{j}}$

time step of temporal cross-correlation function [ms]

the $\mathrm{j}$-th DMD mode

length of the vortex generator [mm]

one-column matrix representing the velocities in the field of view

DMD temporal or spatial evolving matrix

the most-amplified frequency of the turbulent shear layer [Hz]

actuation frequency of the acoustic tube $[\mathrm{Hz}]$

DMD complex frequency

perturbation frequency of the oscillating flap [Hz]

step height of the backward-facing step [mm]

height of the vortex generator [mm]

length of the acoustic tube [mm]

reattachment length behind the backward-facing step

number of anti-node of standing wave in the acoustic tube

DMD propagation matrix

cross-spectral density function of two POD coefficient $a_{i}$ and $a_{j}$

the i-th POD mode

four quadrant events of Reynolds shear stress 


\begin{tabular}{|c|c|}
\hline $\operatorname{Re}_{\mathrm{h}}$ & Reynolds number based on the step height \\
\hline$r_{i, j}$ & radius of two POD coefficient scatter \\
\hline $\mathrm{R}_{\mathrm{ij}}$ & coefficient of two-point cross-correlation function \\
\hline $\mathrm{R}_{\mathrm{L}}$ & reduction rate of reattachment length \\
\hline $\mathrm{r}_{\text {mean }}, \mathrm{r}_{\mathrm{r} . \mathrm{m} . \mathrm{s}}$ & residual of convergence in the field of view \\
\hline $\mathbf{S}$ & similar matrix of the propagation matrix $\mathbf{P}$ \\
\hline SPL & sound pressure level $[\mathrm{dB}]$ \\
\hline $\mathrm{St}_{\mathrm{h}}$ & Strouhal number based on the step height \\
\hline $\mathbf{U}$ & matrix of fluctuating velocity components in POD analysis \\
\hline $\mathrm{u}, \mathrm{v}, \mathrm{w}$ & velocity components in the $\mathrm{X}$-, $\mathrm{Y}$ - and Z-direction \\
\hline $\mathrm{U}_{0}$ & free-stream velocity $[\mathrm{m} / \mathrm{s}]$ \\
\hline $\mathbf{u}_{\mathrm{c}}, \mathbf{U}_{\mathrm{c}}$ & local convection velocity in turbulent shear layer \\
\hline $\mathbf{u}_{\mathbf{i}}$ & column matrix in POD analysis \\
\hline $\mathrm{u}_{\text {r.m.s. }}, \mathrm{v}_{\mathrm{r} \text {.m.s. }}$ & root-mean-square velocities \\
\hline $\mathbf{U}_{\text {svd }}, \Sigma_{\text {svd }}, \mathbf{V}_{\text {svd }}$ & matrices of singular value decomposition \\
\hline $\mathrm{V}_{\text {flap }}$ & velocity of the center portion of the oscillating flap $[\mathrm{m} / \mathrm{s}]$ \\
\hline $\mathbf{v}_{\mathbf{i}}$ & column matrix in DMD analysis \\
\hline $\mathrm{X}, \mathrm{Y}, \mathrm{Z}$ & three-dimensional coordinate system $[\mathrm{mm}]$ \\
\hline$Y_{n}$ & height of the neutral position of oscillating flap [mm] \\
\hline $\mathrm{Y}_{\text {flap }}, \mathrm{V}_{\text {flap }}$ & position and velocity of the oscillating flap $[\mathrm{mm}, \mathrm{m} / \mathrm{s}]$ \\
\hline$\alpha$ & phase angle of the acoustic tube and oscillating flap $\left[{ }^{\circ}\right]$ \\
\hline$\beta$ & angle of incidence of the vortex generator $\left[{ }^{\circ}\right]$ \\
\hline$\delta, \delta^{*}, \theta$ & $99 \%$, displacement and momentum thickness of boundary layer [mm] \\
\hline$v$ & kinematic viscosity of air at $20^{\circ} \mathrm{C}, 1.48 \times 10^{-5}\left[\mathrm{~m}^{2} / \mathrm{s}\right]$ \\
\hline$\rho$ & density of air at $20^{\circ} \mathrm{C}$ and $0.1 \mathrm{MPa}, 1.21\left[\mathrm{~kg} / \mathrm{m}^{3}\right]$ \\
\hline$\sigma$ & standard deviation \\
\hline$\varphi_{\mathrm{i}, \mathrm{j}}$ & phase angle of two POD coefficient scatter $\left[{ }^{\circ}\right]$ \\
\hline$\omega$ & vorticity $[1 / \mathrm{s}]$ \\
\hline
\end{tabular}




$\begin{array}{ll}\omega^{*} & \text { normalized vorticity } \omega^{*}=\omega \cdot h / \mathrm{U}_{0} \\ \mathbf{u}, \overline{\mathbf{u}}, \widetilde{\mathbf{u}}, \mathbf{u}^{\prime} & \text { instantaneous, mean, periodic and random fluctuating velocity vectors } \\ \overline{\mathbf{u}}, \overline{\mathrm{v}}, \overline{\mathrm{w}} & \text { time-averaged velocity components } \\ \lambda_{\mathrm{i}}, \mathbf{A}_{\mathbf{i}} & \text { eigenvalue and eigenvector in POD analysis } \\ \lambda_{\mathrm{i}}, \mathbf{E}_{\mathbf{i}} & \text { eigenvalue and eigenvector in DMD analysis } \\ \tau_{\mathrm{xy}}^{\prime} & \text { Reynolds shear stress } \\ \tilde{\mathbf{C}} & \text { covariance matrix in POD analysis } \\ \overline{\mathbf{U}} & \text { time-averaged flow, mean velocity } \\ \mathbf{V}_{\mathbf{x}}, \mathbf{V}_{\mathbf{x}+\Delta \mathbf{x}} & \text { matrix of fluctuating velocity components in DMD analysis } \\ \langle\mathbf{u}\rangle & \text { phase-averaged velocity vectors }\end{array}$

2D-2C two-dimensional two-component

3D-3C $(\mathrm{t}) \quad$ time-resolved three-dimensional three-component

AT acoustic tube

BFS backward-facing step

B.L. boundary layer

CCD charge-coupled device

CMOS complementary metal-oxide-semiconductor

DEHS di-ethyl-hexyl-sebacate, a liquid for droplet seeding

DLR Deutsches Zentrum für Luft- und Raumfahrt

German Aerospace Center

DMD dynamic mode decomposition

mean average value

MLOS-SMART multiplicative line-of-sight simultaneous multiplicative algebraic reconstruction technique

NACA National Advisory Committee for Aeronautics (1915-1958) 


$\begin{array}{ll}\text { Nd:YAG } & \text { neodym yttrium-aluminum-garnet } \\ \text { OF } & \text { oscillating flap } \\ \text { PIV } & \text { particle image velocimetry } \\ \text { POD } & \text { proper orthogonal decomposition } \\ \text { PTV } & \text { particle tracking velocimetry } \\ \text { r.m.s. } & \text { root-mean-square value } \\ \text { RSD } & \text { relative standard deviation } \\ \text { TBL } & \text { turbulent boundary layer } \\ \text { VG } & \text { vortex generator }\end{array}$




\section{Introduction}

\subsection{Coherent structures in turbulence}

\subsubsection{Discovery and definition}

Investigation of coherent structures in turbulent shear flows has been an attractive subject in fluid mechanics since the 1960s. The early discoveries of these phenomena came from flow visualization experiments. Low-speed streaks were first found in the near-wall regions of a turbulent boundary layer by Kline et al. (1967) by means of the hydrogen bubbles method. The existence of large-scale coherent structures in turbulent mixing layers was investigated by Brown and Roshko (1974). As further studies were carried out on turbulent boundary layers (Corino and Brodkey 1969; Hussain and Reynolds 1970; Kim et al. 1971; Blackwelder and Kovasznay 1972; Falco 1977), free shear layers (Winant and Browand 1974; Brown and Roshko 1974; Hussain and Zaman 1985; Husain and Hussain 1995) and turbulent jets (Crow and Champagne 1971; Zaman and Hussain 1980; Hussain and Zaman 1981), the understanding of turbulence has gradually been changed. Being different from the early understanding that turbulence is essentially a stochastic phenomenon having random fluctuating motions superimposed on a well-defined mean flow (Taylor 1935), it has been gradually realized that large-scale, organized fluid motions play an important role in the momentum and energy transfer processes in turbulent flows (Cantwell 1981). In the early 1980s, applications of 
laser-based optical measurement technology and computational fluid dynamics in fluid mechanics research have just risen. The understanding of the coherent structures in turbulence had increased significantly ever since.

A coherent structure was defined as a connected turbulent fluid mass with instantaneously phase-correlated vorticity over its spatial extent (Hussain 1986). Later, the definition was expanded to other fundamental flow variables, such as velocity component, density and temperature that exhibited positive auto- or cross-correlation values over a range of space or time (Robinson 1991). Such coherent motions play a predominant role in turbulent shear flows such as turbulent boundary layers and free shear layers. However, the forms, scales and organizations of coherent structures vary from flow to flow. For instance, the hairpin-shaped vortices in a turbulent boundary layer and the rolled-up vortices in a plane shear layer exhibit distinctive differences in spatial structures and dynamic features. Despite of the limitations of covering all types of coherent structures in various flow phenomena, these definitions are suitable for characterizing the self-sustained and artificially excited quasi-periodic coherent structures in turbulent shear layers.

\subsubsection{Approaches to coherent structures}

Based on the research progress of turbulent boundary layer, Robinson (1991) summarized four eras of investigating coherent structures as:

(a) the discovery era (1930s-1950s);

(b) the flow visualization era (1960s-early 1970s);

(c) the conditional sampling era (1970s);

(d) the computer-simulation era (1980s-present).

Generally, there have been two basic lines of approach to extract coherent structures out of turbulent flows in the early research. The first method to reveal this phenomenon is flow visualization. Kline et al. (1967) discovered the formation and break-up processes of low-speed streaks in the near-wall region of a turbulent boundary layer and suggested that these processes 
play an important role in production and transport of new turbulence in boundary layers. Corino and Brodkey (1969) observed similar organized motions, as studied by Kline et al. (1967), in the near-wall region of a turbulent pipe flow in details and described the behavior of coherent structures as a sequence of local events, including deceleration, acceleration, shear layer, ejection and sweep. Falco (1977) observed hairpin vortices in the outer region of a turbulent boundary layer and measured the coherent features of the "typical eddies" by hot-wire probes. Besides in boundary layer flows, coherent structures in free shear layers have attracted much attention as well. Although it has been generally accepted that highly regular vortex structures exist in a laminar free shear layer (Freymuth 1966), large-scale coherent structures were also found in a turbulent mixing layer at high Reynolds numbers by Brown and Roshko (1974). These coherent motions evolve in a regular pattern with a growing scale, though small-scale turbulence is superimposed. The flow visualization, in the Lagrangian point of view, forms an ensemble picture of a flow pattern and enables direct experimental observations of complex flow structures and their behavior, which inspires much of the following work on coherent structures. The other method of approach to coherent structures is the calculation of spatial and temporal correlation functions based on hot-wire measurements. Taylor (1936) introduced correlation functions to characterize flow structures in turbulent flows quantitatively. Favre et al. (1957) estimated the convection velocities of turbulent eddies in a turbulent boundary layer based on the temporal displacements of the space-time cross-correlation peaks. Further measurements have also been applied to turbulent shear layers (Wills 1964), pipe flows (McConachie 1981) and cavity flows (Bian et al. 2011). Townsend (1970) suggested "a double-roller structure" in turbulent shear flows as an interpretation of a spatial correlation function. The correlation method, on the other hand, enables to represent coherent structures by a well-defined mathematical description in an Eulerian point of view. However, flow visualization methods as well as temporal and spatial cross-correlation functions are essentially limited by the lack of sufficient quantitative data and therefore can hardly be used to draw a whole picture of coherent structures. 
Since the 1980s, many improvements from qualitative visualizations and descriptions to quantitative measurements of ensembles of velocity vector fields have been made, which is mainly based on the applications of laser techniques and digital computers (Adrain 2005). Particle image velocimety (PIV) techniques have been widely used in the measurements of fundamental research and engineering applications. As an instantaneous, quantitative and non-intrusive measurement method, PIV can obtain the velocity information of a whole flow field and therefore enables researchers to analyze complex structures of coherent motions as well as their interactions. Westerweel et al. (2013) reviewed planar, stereoscopic and tomographic PIV techniques as important tools for investigating complex and turbulent flows. He summarized the new directions of PIV techniques for both the underway and future development, which include:

(a) the measurement of three-dimensional three-component velocity vector fields, referred to as tomographic PIV;

(b) the use of high-repetition-rate laser pulses and high-speed cameras, referred to as time-resolved PIV;

(c) the single-pixel resolution of average and root-mean-square velocities, referred to as statistical PIV;

(d) the measurement of fluid acceleration by using multiple laser pulses and particle image recordings, referred to as multi-pulse PIV.

Recently, the four-dimensional particle tracking velocimetry method "Shake-The-Box" has been developed (Schanz et al. 2013; Schanz et al. 2014) which combines the advantages of Lagrangian particle track reconstruction delivering velocity and acceleration measures at high positional accuracies with those of the high spatial resolution of a tomographic PIV system delivering the complete time-resolved velocity gradient tensor. Turbulent flow research can greatly benefit from recent developments in the field of particle based measurement techniques (Schröder et al. 2015).

\subsubsection{Coherent structures in turbulent shear flows}

In the 1960s-1970s, flow visualization played an important role in the early investigations on 
coherent structures. Freymuth (1966) found highly regular vortices in the transitional process of a separated laminar boundary layer by flow visualization. The pairing between two spanwise vortices is closely associated with the growth of such shear layer (Winant and Browand 1974). Brown and Roshko (1974) discovered large-scale vortical structures superimposed on fine-scale turbulence background in a turbulent mixing layer. With an increasing Reynolds number, small and smaller scales of motion appeared while the coherent structures remained as a permanent feature. These coherent structures exhibit in a regular pattern with an increasing wavelength and amplitude through the turbulent mixing layer and eventually give way to random turbulent fluctuations further downstream. More and more research focused on this "quasi-order structure", which was described as "the new trend" by Laufer (1975), as well as its interaction with random turbulent motions. Hussain and Zaman (1981) found a "preferred mode" of an axisymmetric turbulent jet, which was generated by artificial perturbations at a selected frequency. The background turbulence was highly organized: the saddle point region between two vortices exhibits higher incoherent Reynolds shear stress but lower vorticity and lower turbulent kinetic energy, which is totally opposite to the vortex center. Cantwell (1981) also found this phenomenon by examining the vorticity and cross-correlation coefficient derived from the fluctuating velocity components and concluded that "the background turbulence is neither small nor random" in his review article. Hussain (1986) drew a clear cross-sectional schematic diagram of the essential contours of a coherent structure in his summary of studies of free shear layers. A coherent structure contains a vortex center, two saddles and essential contours of coherent intermittency, incoherent turbulence intensities, incoherent Reynolds stress, coherent strain rate and coherent production of turbulence. Although models of mechanisms of coherent structures remain controversial and descriptions of these phenomena vary, it has been widely accepted that "the understanding of coherent structures clearly holds the key to understanding turbulence management and control" (Hussain 1986).

With the development of PIV and other optical measurement techniques, a large number of experimental investigations have been carried out aiming at a whole view of spatial features and 
temporal behaviors of coherent structures in turbulent boundary layers (Schröder et al. 2008; Schröder et al. 2011; LeHew et al. 2013; Gao et al. 2013) and turbulent shear flows (Kit et al. 2005; Brun et al. 2008; Bade et al. 2010; Violato et al. 2011; Schröder et al. 2013). Kit et al. (2005) reconstructed clear patterns of spanwise Kelvin-Helmholtz waves and streamwise vortices in a perturbed mixing layer based on phase-locked stereoscopic PIV data. These two types of coherent structures were emanating from primary and secondary instabilities of the shear layer. Brun et al. (2008) identified the frequency signatures of von Kármán and Kelvin-Helmholtz vortices in a separated shear layer downstream of a square cylinder by laser doppler velocimetry. Bade et al. (2010) studied the contribution of large-scale coherent structures in a free shear layer by planar PIV. The characterizations of the coherent structures, such as spacing, size and strength, are discussed. In the past decade, tomographic particle image velocimetry has been noticeably developed to provide volumetric velocity measurements of fluid motions (Elsinga et al. 2006). This measurement technique is able to obtain a sequence of instantaneous three-component velocity vector fields within a three-dimensional volume, which is thereby suitable for analysis of complex vortex structures in turbulent shear flows. Violato et al. (2011) studied three-dimensional circular vortices in a transitional jet flow by time-resolved tomographic PIV. The shedding and pairing of vortices were all clearly shown. Schröder et al. (2013) visualized hairpin-like vortices in transitional shear layer downstream of a backward-facing step by high-resolution tomographic PIV. The complex topologies of the streamwise hairpin-like vortices and classical spanwise vortices are revealed by instantaneous velocity vector fields. Although the understanding of coherent structures has been improved with the help of the advanced techniques, however, raised questions are still more than answers.

\subsection{Flow control research}

Flow control techniques are to change the state of fluid motions by applying force, fluid mass, heat and other methods to the flow. It is a branch of fluid mechanics and a multidisciplinary field of mechanics, acoustics, electromagnetics, control theory, etc. Flatt (1961) gave a definition of the flow control of wall-bounded flows: "Boundary layer control includes any 
mechanism or process through which the boundary layer of a fluid flow is caused to behave differently than it normally would". This definition can also be extended to free-shear flows. If an unknown fluid phenomenon is viewed as a "black-box" problem, then the contents of the "black-box" may be studied by changing various "inputs" and studying the resulting "outputs" (Clauser 1956). Flow control provides a key to revealing complex flow system and is capable of improving the understanding of the underlying physical mechanism.

\subsubsection{Origin and development}

The history of flow control research dates back to the early 20th century. When Prof. Ludwig Prandtl (1875-1953) and other scientists and designers in the field of fluid mechanics, later referred to as "the Göttingen School", laid the foundation of the boundary layer theory, they also applied active flow control techniques to boundary layer flows in order to avoid separation (Schlichting 1979). In the early research, the flow control methods mainly include:

(a) Motion of solid walls (Ackeret 1925; Prandtl 1925);

(b) Blowing and suction of boundary layer (Prandtl 1904; Schrenk 1935);

(c) Injection of different gas (Gross et al. 1961; Faulders 1961);

(d) Laminar airfoil to delay transition to turbulence (Doetsch 1940; Wortmann 1969);

(e) Cooling of wall (Linke 1942; Liepmann and Fila 1947).

These methods had been widely applied to the flows on a flat plate, around a circular cylinder and an airfoil. Some books (Lachmann 1961; Schlichting 1979; Gad-el-Hak et al. 1998) have summarized the scientific research and engineering applications of those fundamental flow control approaches.

The flight of birds offers a rich source of inspiration and ideas for the development of flow control technology. Due to the biological evolution for at least one hundred million years, the birds have adopted sophisticated wings and amazing skills of manipulating complex flows in the air. For instance, eagles use "alula", which are small feathers at the leading-edge of the wings and can be moved upwards and forwards, to increase the lift force and prevent stall when flying at a low speed such as landing (Försching and Hennings 2012). Also, eagles use "wing tip sails", 
which is consisted of a few discrete feathers, to reduce the strength of wing tip vortices and increase flying efficiency (Spillman 1978). Owls have fine-scale feathers as serrations on wing surfaces to reduce noise and fly silently (Bushnell 1991).

Nowadays, various types of flow control techniques have been implemented on aircraft wings and are playing an essential role in aircraft performance, in particular, in the processes of taking-off and landing. For instance, leading-edge slats and trailing-edge flaps can increase the lift coefficient of a wing and therefore provide additional lift force during taking-off and landing. A winglet on the tip of a wing can attenuate the strength of wingtip vortices and increase flow efficiency. Vortex generators on the upper side of a wing or strakes at the nacelle can delay or eliminate flow separation by producing longitudinal vortices in order to increase the momentum transfer between an outer flow and the wing boundary layer. With increasing demands of more efficient air transportation and less carbon emission since the 1970s, the flow control technology is capable of providing additional approaches to further improve aircraft performance.

\subsubsection{Classifications of flow control}

Flow control techniques can be mainly classified into two groups, active and passive flow control, based on a criterion of the energy source (Figure 1- 1). Active flow control uses external energy and usually requires actuators which convert the external energy into the controlled flow. Actuators have various types including fluidic jets, moving objects, acoustic devices, plasma generators and other electromagnetic devices (Cattafesta and Sheplak 2011). On the other hand, passive flow control does not use external energy sources. Some passive control devices, for instance, vortex generators, wing fence and winglets, are fixed on the wall. Other passive control methods, for instance, roughness, longitudinal grooves and flexible wall, use modifications of surfaces to change the flow. An extensive review of active and passive flow control was given by Choi et al. (2008).

Another classification is based on the flow control purpose. The main groups include flow separation control, drag reduction, lift enhancement, control of pressure fluctuations (or referred 
to as noise reduction), maximization or minimization of heat and mass transfer and so on (Bushnell and McGinley 1989). From the point of view of classical control theory, flow control can be classified into open-loop and close-loop types, which depends on whether feed-forward or feed-back techniques are used or not (Moin et al. 1994).

Energy
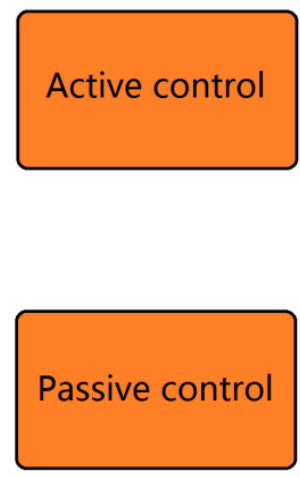

Purpose

Separation control

Drag reduction

Lift enhancement

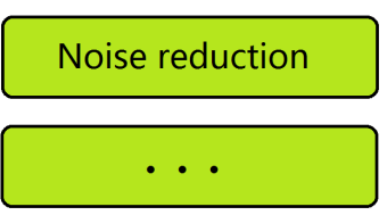

Classical

Control Theory
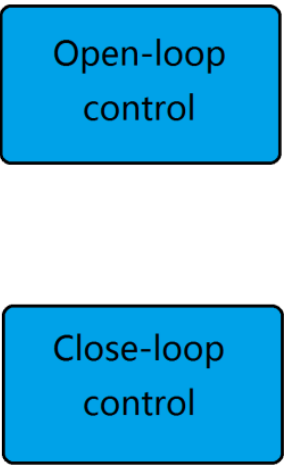

Figure 1- 1 Classifications of flow control techniques

\subsubsection{Flow separation control}

The total drag (also referred to as profile drag) on a solid body in a fluid flow consists of skin friction and pressure drag (also referred to as form drag) (Schlichting 1979). As a major part of the total drag, the pressure drag is caused by the presence of pressure differences around a solid body and is therefore equal to the integral of normal forces. Particularly, when flow separation occurs, the pressure drag increases significantly. For instance, the lift and drag coefficients of a two-dimensional NACA 0012 airfoil (Jacobs et al. 1937; Goett et al. 1939) are plotted in Figure 1- 2. If the angle of attack increases over the critical values, which are equal to $\alpha_{\text {crit }}=10.5^{\circ}$ at $\operatorname{Re}$ $=1.0 \times 10^{5}$ and $\alpha_{\text {crit }}=15.5^{\circ}$ at $\operatorname{Re}=1.0 \times 10^{6}$, the lift coefficients drop down from the maximum values and, at the same time, the drag coefficients increase tremendously. This phenomenon is referred to as stall. Therefore, flow separation control plays an important role in pressure drag reduction. 

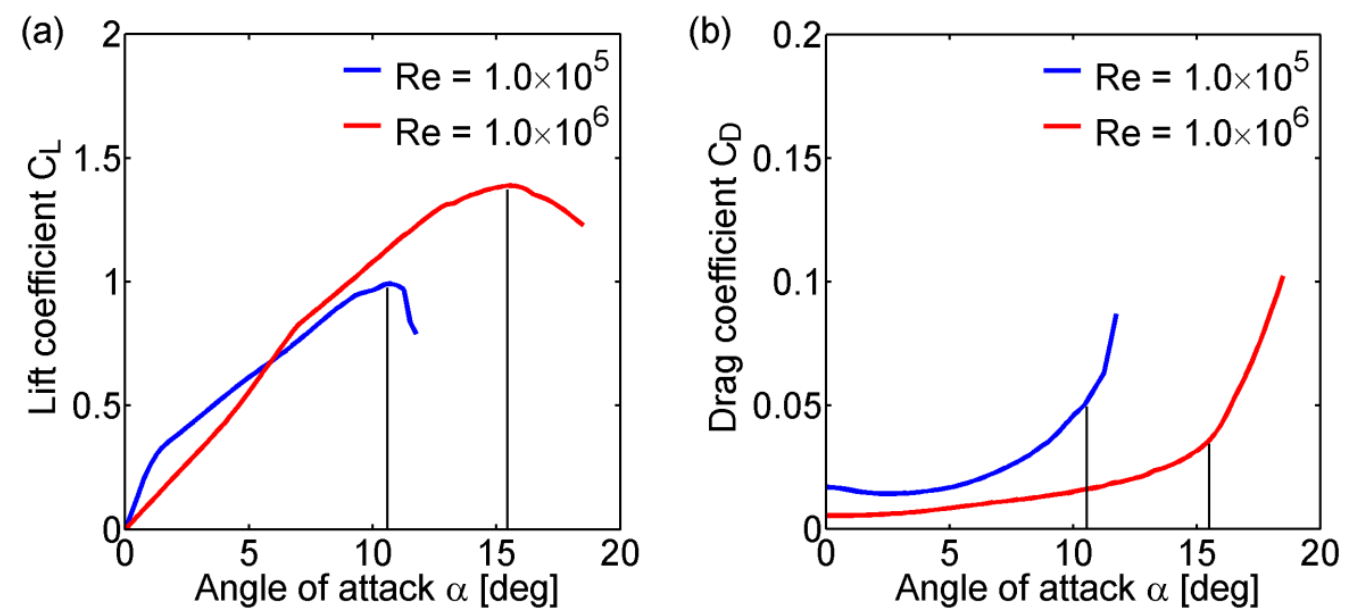

Figure 1- 2 Aerodynamic characteristics of a two-dimensional NACA 0012 airfoil (Jacobs et al. 1937; Goett et al. 1939). (a) lift coefficient; (b) drag coefficient. The critical angles of attack are marked by the black lines.

Among various types of flow control techniques, vortex generators (VGs) have been widely used in flow separation control in scientific research and engineering applications since the 1940s (Taylor 1947). As economical and reliable passive devices, VGs are designed in various types, such as vane, ramp, doublet, wishbone and wedge (Lin 2002), and are used to generate small vortices in order to increase the momentum transfer between outer flows and boundary layers. Conventional VGs have a device height $\mathrm{H}$ in the order of the boundary layer thickness $\delta$ as $\mathrm{H} \approx \delta$ (Schubauer et al. 1960). Since the 1970s, low-profile VGs have been extensively investigated due to the ability of high flow control effectiveness and less drag induction (Kuethe 1972). Low-profile VGs have a device height $\mathrm{H}$ of only a fraction of the boundary layer thickness $\delta$ as $\mathrm{H}<\delta$ and are thus submerged within turbulent boundary layers. They are also referred to as "micro VGs", "sub-boundary-layer VGs" or "submerged VGs" in the early literature. Among various designs, wedge-shaped VGs are devices of triangular planform with the top surface facing upstream (forward orientation) or downstream (backward orientation), which generate counter-rotating streamwise vortices from the two swept side edges (Rao et al. 1988). Betterton et al. (2000) found that the streamwise decay of vortex strength downstream of wedge-type VGs is much higher than those of other types, such as single vane and joined vanes. 
He concluded that this phenomenon is caused by the close proximity and interaction of the counter-rotating vortices. Ashill et al. (2001) applied various types of VGs on a zero-pressure-gradient flat plate and found that backward wedges had the highest vortex decay rate in the downstream distance of $15 \cdot \mathrm{H}$ among various types of VGs, for instance, joined and spaced counter-rotating vanes, forward- and backward-wedges. Moreover, he found that the streamwise vortices produced by the backward wedges were closer to the wall than for the other devices. Therefore, the faster vortex decay downstream of the backward wedge could be explained by the stronger influence of wall shear resulting in further attenuation of the vortex strength. The wedge-type VGs also achieved a higher lift-to-drag ratio than other types in transonic airfoil flow separation control. Lin (2002) provided an in-depth review of the experimental investigations and summarized that low-profile VGs are capable of providing sufficient vertical momentum transfer over a region several times higher than their own height, and moreover, causing less device drag. Due to the low-profile and symmetric designs, the counter-rotating streamwise vortices are generated from the two side edges of each VG and embedded closely to the wall. The vortex characteristics of the backward wedge-type VGs, which include the counter-rotating mutual interaction, submergence within boundary layers and high vortex decay rate, improve the momentum transfer between the outer flow and the boundary layer advantageously.

Although the backward wedge-type VGs on flat plates and airfoils have been extensively studied (Lin 2002), the structure and coherent features of VG-induced streamwise vortices in a turbulent separated shear layer over a backward-facing step has not been fully revealed. Previous experimental investigation has shown that low-profile wedge-type VGs are able to increase the Reynolds stress and turbulent kinetic energy in separated shear layers and then result in $29.1 \%$ reduction of the separation length downstream of the BFS (Ma et al. 2014b). However, the counter-rotating streamwise vortices superimposed on the classical spanwise oriented two-dimensional waves (Schröder et al. 2013) lead to an even more complex flow field. The Chapter 6 focuses on uncovering the complex vortex structures and their coherent features. 


\subsection{Backward-facing step flow}

A turbulent backward-facing step (BFS) flow is a multi-scale flow phenomenon, which contains a separated turbulent shear layer, recirculation region and a reattachment area. The sketch of the BFS flow is shown in Figure 1- 3. Generally, a backward-facing step flow has some similar characteristics to a plane-mixing layer (Brown and Roshko 1974; Chandrsuda et al. 1978). For instance, after separating from a fixed edge, both of the shear layers grow gradually and contain two-dimensional vortices due to the Kelvin-Helmholtz instability. Being different from a plane-mixing layer, however, a backward-facing step flow has a curved dividing streamline (indicated by the dashed line) apparently toward the wall, and the low-speed flow under the shear layer forms the recirculation region due to the presence of the walls (Eaton and Johnston 1981).

A two-dimensional BFS provides a typical flow separation case with a fixed separation edge, so it has been widely used as a fundamental flow case by many researchers for flow separation control studies. A primary goal of most flow separation control strategies is to reduce the reattachment length $\mathrm{L}$, which is defined as the distance from the step to where the mean flow reattaches on the plate surface. A reduction rate is defined as:

$$
\mathrm{R}_{\mathrm{L}}=\frac{\mathrm{L}_{0}-\mathrm{L}_{\mathrm{c}}}{\mathrm{L}_{0}} \times 100 \%
$$

which is frequently used to evaluate the flow control effectiveness. Although the BFS has a simple geometry, the separated and reattached flow is still complex. The reattachment length is highly dependent on initial and boundary conditions, such as incoming boundary layer characteristics and step height. The separated shear layer exhibit nonlinear growth at preferred frequencies and interact with the recirculation region. If the incoming boundary layer is laminar, laminar-turbulent transition occurs soon after the separation, unless the Reynolds number is very low. If the incoming flow is fully turbulent, the flow separation is independent of the Reynolds number (Eaton and Johnston 1981). As the Reynolds number increases, the reattachment length converges to approximately seven to eight times of the step height while 
small- and smaller-scale vortices evolve in addition to large-scale flow separation, which makes the flow field even more complex.

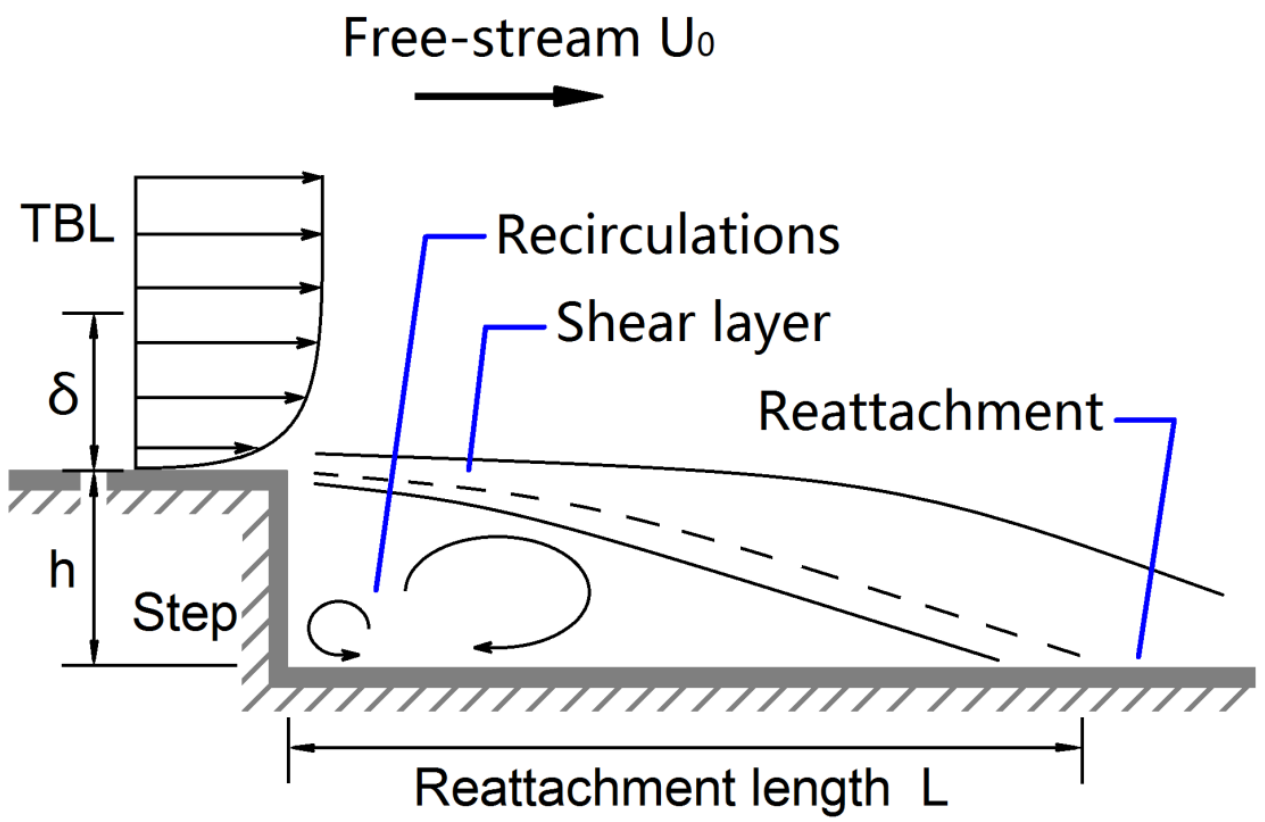

Figure 1- 3 Schematic diagram of turbulent backward-facing step flow

A large number of investigations have been carried out on turbulent BFS flows. Eaton and Johnston (1981) provided an extensive review of experimental studies on BFS flows and discussed the influence of various parameters on flow separation and reattachment, such as initial boundary layer state (laminar or turbulent), ratio of boundary layer thickness to step height, aspect ratio of step width to height and other parameters. Armaly et al. (1983) investigated laminar, transitional and turbulent BFS flows in a two-dimensional channel at $70<$ $\operatorname{Re}<8000$ by experiments. It was concluded that, if the boundary layer was fully turbulent, the reattachment length lied around $7<\mathrm{L} / \mathrm{h}<8$ and became independent of Reynolds number. Active control devices were applied to reduce reattachment lengths by many researchers. The Strouhal number based on the step height is defined as:

$$
\mathrm{St}_{\mathrm{h}}=\frac{\mathrm{f} \cdot \mathrm{h}}{\mathrm{U}_{0}}
$$

which is frequently used as a non-dimensional perturbation frequency. Bhattacharjee et al. (1986) used a loudspeaker above the BFS to influence the flow and found that the effective forcing was 
in a range of $\mathrm{St}_{\mathrm{h}}=0.2-0.4$. Chun et al. (1996) applied a sinusoidal oscillating jet at the separation edge in a BFS flow at $\mathrm{Re}=23,000$. It was found that an effective perturbation at $\mathrm{St}_{\mathrm{h}}$ $=0.29$ reduced the reattachment length by $35 \%$. The same effective non-dimensional perturbation frequency $\mathrm{St}_{\mathrm{h}}=0.29$ was also proposed by Roos et al. (1986) by using an oscillating flap at the step edge. Besides, another important parameter is the Strouhal number based on the momentum thickness of the boundary layer, which is defined as:

$$
\mathrm{St}_{\theta}=\frac{\mathrm{f} \cdot \theta}{\mathrm{U}_{0}}
$$

Hasan (1992) found two distinct modes of instability in the shear layer downstream of a BFS. The one is called the "shear layer mode", which is governed by the Kelvin-Helmholtz instability. The other one is named the "step mode", which consists of an interaction between the shear layer and the recirculation region. Sigurdson (1995) described these two different instabilities from the point of view of vortex interactions. The Kelvin-Helmholtz instability consists of an interaction of the shear layer vorticity with itself; the vortex shedding instability, on the other hand, consists of an interaction of the shear layer with the wall. Comparison of the present BFS flow configuration with the literature is listed in Tabel 1- 1. Because of the complexity of the flow separation and vortex interaction, a complete understanding of the physical mechanism of turbulent separation and reattachment has still not been obtained.

In active flow control research, the perturbation frequency is an essential parameter of a flow control actuator. In order to determine the optimal frequency, Sigurdson (1995) classified the perturbation frequencies $f_{p}$ into four regimes, which were based on the initial Kelvin-Helmholtz frequency $\mathrm{f}_{\mathrm{KH}}$ and the fundamental frequencies $\mathrm{f}_{0}$ (also referred to as the most-amplified frequency) of the separated shear layer:
(a) $\mathrm{f}_{\mathrm{KH}} \ll \mathrm{f}_{\mathrm{p}}$
(b) $\mathrm{f}_{0} \ll \mathrm{f}_{\mathrm{p}} \leq \mathrm{f}_{\mathrm{KH}}$
(c) $\mathrm{f}_{\mathrm{p}} \approx \mathrm{f}_{0}$
(d) $\mathrm{f}_{\mathrm{p}} \ll \mathrm{f}_{0}$ 
In general, perturbations in the regime (a) and (d) have little effect and perturbations in the regime (b) can be amplified by the shear layer. The regime (c) indicates a perturbation frequency in the order of the fundamental frequency of the shear layer, in which the shedding instability is primarily driven and the maximum effect in terms of an increase of Reynolds stresses can be achieved. Periodic perturbations which are equal or close to the fundamental frequencies $\mathrm{f} \approx \mathrm{f}_{0}$ have been extensively investigated by many researchers in order to achieve the maximum effect in BFS flows (Roos et al. 1986; Bhattacharjee et al. 1986; Hasan 1992; Chun et al. 1996; Ma et al. 2015), as listed in Tabel 1- 2. Besides, subharmonic perturbations as $\mathrm{f} \approx \mathrm{f}_{0} / 2$ can also influence the shear layer considerably. Ho and Huang (1982) discovered that a planar mixing layer was greatly manipulated by subharmonic forcing at $f \approx f_{0} / 2$. They also found that if only subharmonic waves act, large vortices are formed and no vortex merging occurs. Chao et al. (1990) investigated jet flows with excitations at $\mathrm{f}_{0} / 2$ and $\mathrm{f}_{0}$ independently. Flow visualization showed the fundamental vortices persisted further downstream in the jet direction while the subharmonic vortices grow wider perpendicular to the jet direction. Husain and Hussain (1995) investigated the subharmonic resonance phenomenon in a laminar planar shear layer. They used controlled forcing at the fundamental frequency $\mathrm{f}_{0}$, its subharmonic $\mathrm{f}_{0} / 2$ and the combination of both $\mathrm{f}_{0}+\mathrm{f}_{0} / 2$, respectively, and found that subharmonic waves were closely related to vortex pairing. Although great efforts have been made on this flow phenomenon, a complete understanding of the physical mechanism of subharmonic vortices and their influence on the development of turbulent shear layer has still not been obtained. Previous experimental results have shown that acoustic perturbations at the fundamental frequency in turbulent BFS flow can be amplified in the shear layer and achieve an apparent reduction of the reattachment length. Therefore, in the present work the investigation of the oscillating flap focuses on the influence of small subharmonic perturbations in the large-scale separated shear layer downstream of a BFS. A hierarchy of the spatial scales in the present study is listed in Tabel 1- 3 and the detailed parameters are given in the following chapters. 
Tabel 1- 1 Comparison of the present BFS flow and the literature

\begin{tabular}{lllllllll}
\hline \hline Author(s) & $\mathrm{h}[\mathrm{mm}]$ & $\delta / \mathrm{h}$ & $\theta / \mathrm{h}$ & Aspect ratio & $\mathrm{Re}_{\mathrm{h}}$ & $\mathrm{L}_{0} / \mathrm{h}$ & B.L. \\
\hline Roos et al. (1986) & 89 & 0.38 & $/$ & 10 & 20,000 & 5.5 & turb. \\
Bhattacharjee et al. (1986) & 56 & $/$ & 0.018 & 16.25 & 45,000 & 6.0 & turb. \\
Hasan (1992) & 30 & 0.15 & 0.023 & 20 & 11,000 & $/$ & lam. \\
Chun et al. (1996) & 50 & 0.38 & 0.036 & 12.5 & 23,000 & 7.20 & turb. \\
Ma et al. (2015) & 30 & 0.53 & 0.066 & 43.3 & 20,000 & 7.1 & turb. \\
Present & 30 & 0.53 & 0.066 & 43.3 & 20,000 & $\mathbf{7 . 1}$ & turb. \\
\hline \hline
\end{tabular}

Tabel 1- 2 Flow control results of present study and the literature

\begin{tabular}{llllllll}
\hline \hline Author(s) & $\mathrm{h}[\mathrm{mm}]$ & $\mathrm{Re}_{\mathrm{h}}$ & $\mathrm{St}_{\mathrm{h}}$ & $\mathrm{L}_{0} / \mathrm{h}$ & $\mathrm{L}_{\mathrm{c}} / \mathrm{h}$ & $\mathrm{R}_{\mathrm{L}}$ & perturbation \\
\hline Roos et al. (1986) & 89 & 20,000 & 0.29 & 5.5 & 4.5 & $18.2 \%$ & mechanical \\
Bhattacharjee et al. & 56 & 45,000 & 0.28 & 6.0 & 5.0 & $16.7 \%$ & acoustic \\
$\begin{array}{l}\text { (1986) } \\
\text { Hasan (1992) }\end{array}$ & 30 & 11,000 & $0.14-0.85$ & $/$ & $/$ & $/$ & acoustic \\
Chun et al. (1996) & 50 & 23,000 & 0.29 & 7.20 & 4.68 & $35.0 \%$ & acoustic \\
Ma et al. (2015) & 30 & 20,000 & 0.3 & 7.1 & 4.0 & $43.7 \%$ & acoustic \\
$\quad$ AT & 30 & 20,000 & 0.3 & 7.1 & 4.0 & $\mathbf{4 3 . 7 \%}$ & acoustic \\
Present OF & 30 & 20,000 & 0.165 & 7.1 & 4.9 & $\mathbf{3 1 . 0 \%}$ & mechanical \\
$\quad$ VGs & 30 & 20,000 & $/$ & 7.1 & 5.03 & $\mathbf{2 9 . 1 \%}$ & passive \\
\hline \hline
\end{tabular}

Tabel 1- 3 Hierarchy of spatial scales of the present BFS flow

\begin{tabular}{ll}
\hline \hline Spatial scale & Length $[\mathrm{mm}]$ \\
\hline Reattachment length of the BFS flow & 211.5 \\
Backward-facing step height & 30 \\
Thickness of the turbulent boundary layer before separation & 16 \\
Height of the vortex generators & 10 \\
Amplitude of the perturbations of oscillating flap & 5 \\
Width of the outlet of the acoustic tube & 2 \\
Vector spacing of high-resolution 2D-2C PIV & 0.3 \\
\hline \hline
\end{tabular}




\subsection{Outline}

This dissertation presents the investigation of coherent structures generated by the interaction of active and passive flow control devices with turbulent flow separation downstream of a backward-facing step. The following questions are to be answered:

(a) How are the quasi-periodic coherent structures generated by the artificial perturbations?

(b) How do these coherent structures change the turbulent shear layer?

(c) What are the flow separation control effects of these coherent structures?

This dissertation is organized as follows.

In Chapter 2, the experimental apparatus and procedure is presented. The designs of the flow control devices together with the planar and tomographic PIV measurements are described.

In the following Chapter 3, the post-processing methods are discussed. Velocity triple decomposition, quadrant analysis, two-point cross-correlation functions, proper orthogonal decomposition (POD) and dynamic mode decomposition (DMD) are used to extract underlying coherent structures out of the turbulent shear flow from spatial and temporal points of view.

In Chapter 4-6, the flow control results of the acoustic tube, oscillating flap and vortex generators are shown and discussed, respectively. The attention is focused on device-generated coherent structures and the resulting increased Reynolds shear stresses.

In Chapter 7, the conclusions of this dissertation are given and a brief outlook of further research is discussed.

"Turbulence is the most important unsolved problem of classical physics."

Richard P. Feynman (1918-1988) 


\section{Experimental apparatus and procedure}

\subsection{Flow facility}

The experiments were carried out in the 1m-low-speed wind tunnel at the German Aerospace Center (DLR) in Göttingen, Germany (Figure 2- 1). The open test section was 1,400 mm long and had a rectangular cross-section of $1,050 \times 700 \mathrm{~mm}^{2}$. The free-stream velocity was $\mathrm{U}_{0}=10$ $\mathrm{m} / \mathrm{s}$ with a turbulence level of $\sim 0.15 \%$. A backward-facing step (BFS) model was mounted horizontally on a flat plate with an elliptical leading edge. The model was placed in the open test section without side or top plates. The BFS model was $900 \mathrm{~mm}$ long, including a ramp of $4.3^{\circ}$ inclination and $400 \mathrm{~mm}$ length in the flow direction, and $1300 \mathrm{~mm}$ wide in the spanwise direction with a step height of $\mathrm{h}=30 \mathrm{~mm}$. The aspect ratio of width to height was 43.3 , which is larger than the two-dimensionality criterion of 10 (de Brederode and Bradshaw 1978) for assuming a two-dimensional mean flow in the center portion of the step. The incoming boundary layer was tripped at the leading edge by spanwise zigzag bands with a thickness of 0.4 $\mathrm{mm}$ in order to generate a turbulent boundary layer, which had a thickness of $\delta \approx 16.0 \mathrm{~mm}(\delta / \mathrm{h}$ $\approx 0.53$ ), a displacement thickness of $\delta^{*} \approx 2.8 \mathrm{~mm}$ and a momentum thickness of $\theta \approx 2.0 \mathrm{~mm}$ (shape factor of $\delta^{*} / \theta \approx 1.4$ ) at the BFS (Ma et al. 2015). The Reynolds number, based on the free-stream velocity and the step height, was:

$$
\operatorname{Re}_{\mathrm{h}}=\frac{\mathrm{U}_{0} \cdot \mathrm{h}}{v}=2.0 \times 10^{4}
$$


In the present paper, the flow case with a flow control device is denoted as a "controlled case" while the one without any device is denoted as a "clean case".



Figure 2- 1 1m-low-speed wind tunnel at the German Aerospace Center in Göttingen, Germany

\subsection{Acoustic tube}

An acoustic tube was designed and integrated at the BFS model (Figure 2- 2). The acoustic tube consisted of a spanwise tube of $1=1,700 \mathrm{~mm}$ length with a square cross-section of $30 \times 30 \mathrm{~mm}^{2}$. A thin slot of $150 \times 2 \mathrm{~mm}^{2}$ was placed parallel and near the separation edge with its exit in the flow direction (Figure 2- 3). The acoustic tube was equipped with a loudspeaker (max. 100 Watt, $8 \Omega$ ) installed on one side while the other side was sealed by a hard surface in order to avoid energy leakage. When single-frequency sound waves propagate along the tube, interference occurs between the incident and reflected sound waves. If the half-wavelength fits the length of the tube, standing waves are created. The air column inside the tube is compressed on both sides like an air spring, resulting in periodic blowing and suction through the slot at the same frequency of the actuation. The zero-mass blowing and suction perturbs the shear layer directly. Although the standing wave has a spanwise distribution, the length of the slot was only $8.8 \%$ of the half wavelength. So the actuation at the slot can be considered to be approximately 
uniform along the slot. Because of complexity of the pressure field inside the tube and unknown transfer function of the slot, the acoustic tube calibration provides the relation between the input signal into the loudspeaker and the output SPL at the exit of the slot (Figure 2- 4). It is shown that all curves exhibit linearity at SPL $=110 \mathrm{~dB}$ and show saturations at the highest SPL.

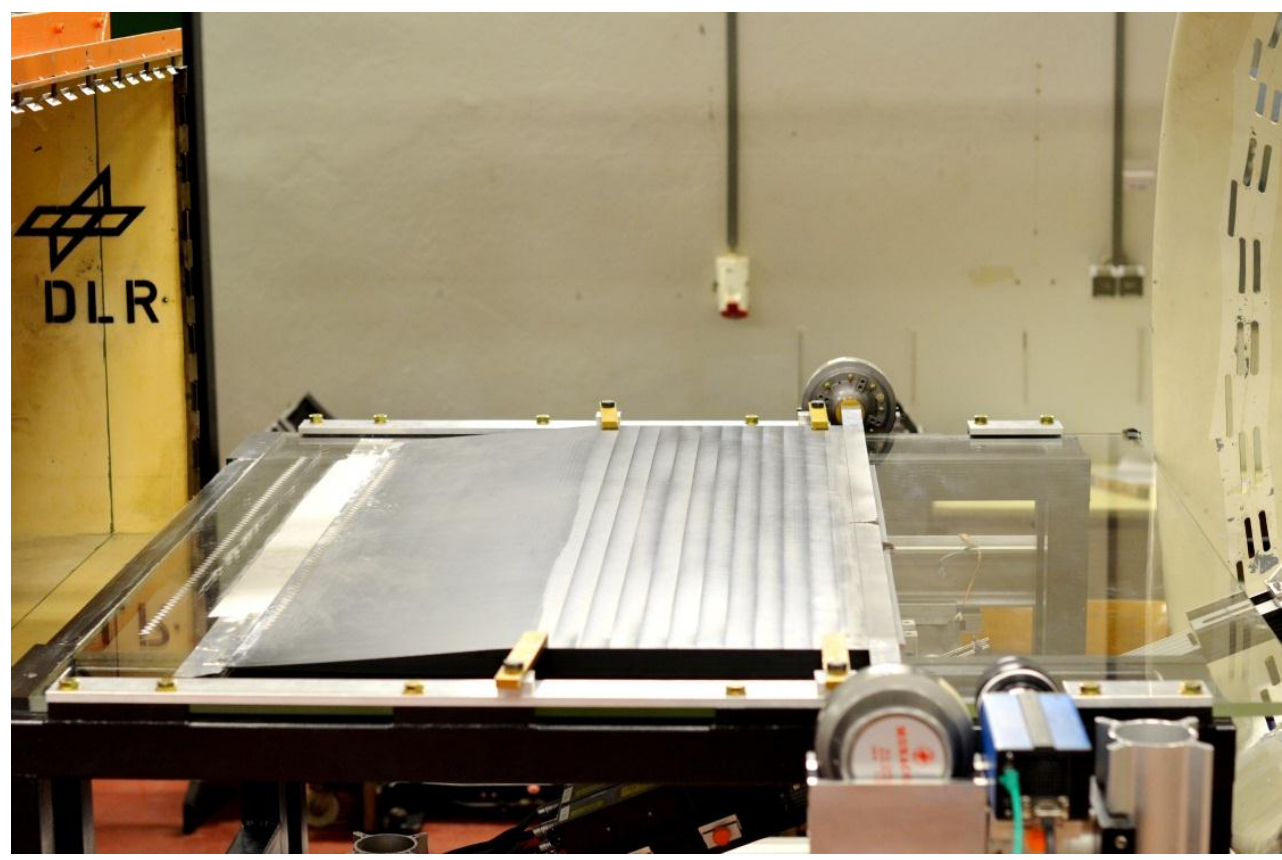

Figure 2- 2 Photograph of the acoustic tube implemented with the backward-facing step

Front view :
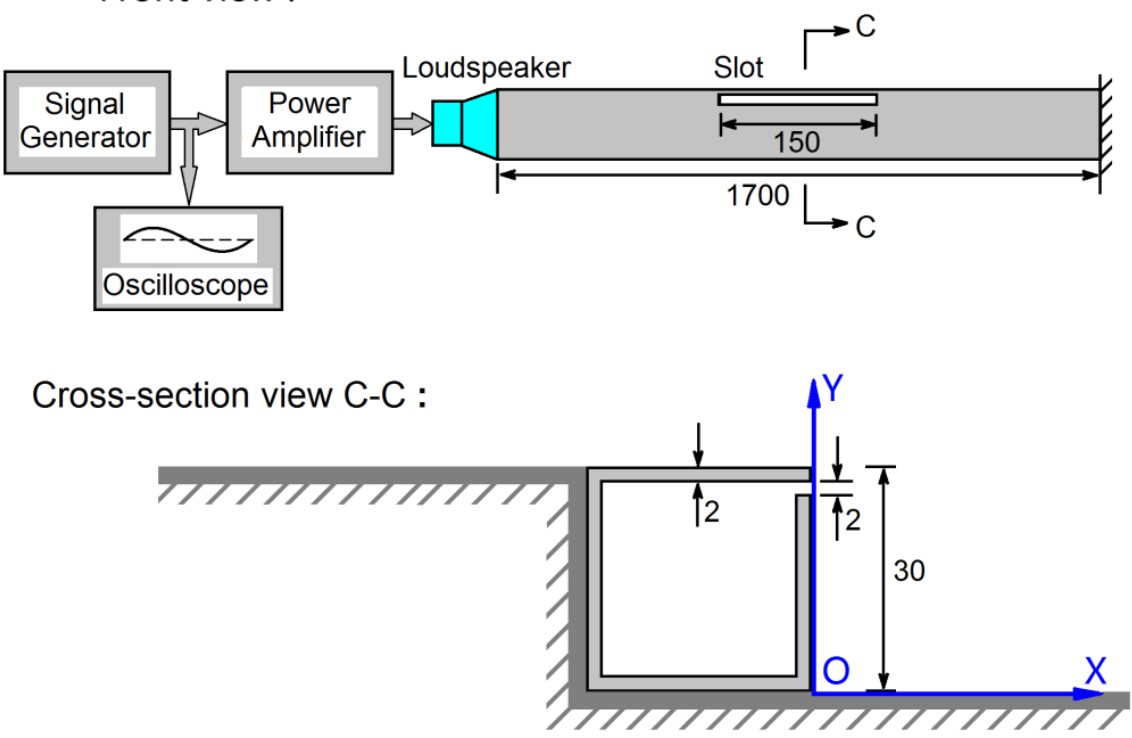

Figure 2- 3 Schematic diagram of acoustic tube integrated with backward-facing step 


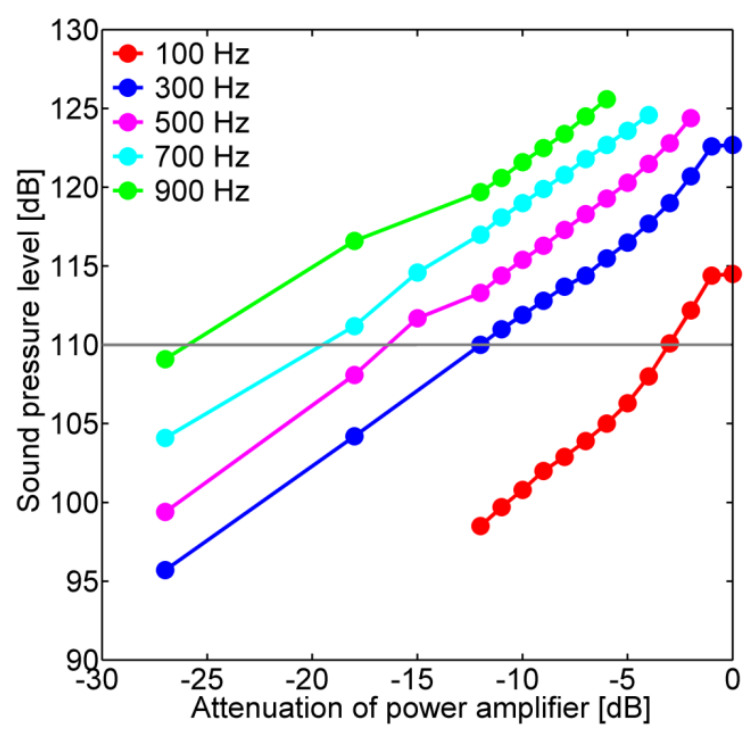

Figure 2- 4 Calibration of acoustic tube. The input is the amplified signal to the loudspeaker. The output is the sound pressure level at the exit of the acoustic tube.

The actuation frequencies $\mathrm{f}_{\mathrm{a}}$ was chosen as:

$$
\mathrm{f}_{\mathrm{a}}=\mathrm{n} \times \frac{\mathrm{c}}{2 \times \mathrm{l}}, \mathrm{n}=1,3,5,7,9
$$

$\mathrm{c}=343 \mathrm{~m} / \mathrm{s}$ is the speed of sound in air at the temperature of $20^{\circ} \mathrm{C}$. The control signals of sine waves were generated by an Agilent waveform generator and then amplified by a Dynacord CL1600 power amplifier. When the actuation frequency was $\mathrm{f}_{\mathrm{a}}=100 \mathrm{~Hz}$, outward acoustic actuations at the exit of the slot were measured by a mobile NTI microphone for acoustic calibration. The frequency spectrum indicates that the output actuations contain the fundamental frequency $f_{a}$ as well as the overtones at higher frequencies (Figure 2- 5). In the parameter study, five actuation frequencies $\mathrm{f}_{\mathrm{a}}=100,300,500,700,900 \mathrm{~Hz}$ and three sound pressure levels SPL $=105,110,115 \mathrm{~dB}$ at the exit of the slot were tested in order to find the parameter influences on the reattachment length. The parameter sets are listed in Table 4- 1. 


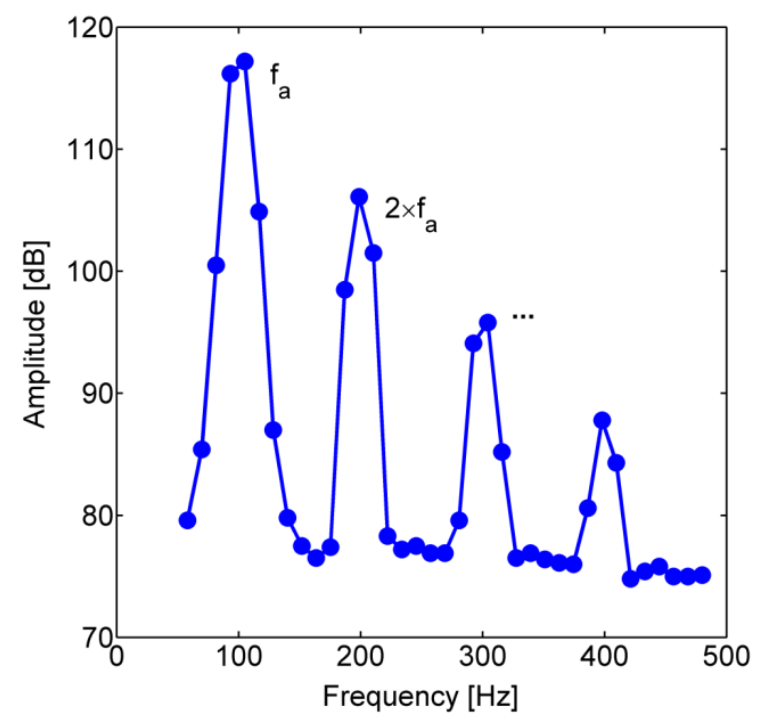

Figure 2- 5 Frequency spectrum of acoustic actuation at $\mathrm{f}_{\mathrm{a}}=100 \mathrm{~Hz}$ and $\mathrm{SPL}=110 \mathrm{~dB}$

\subsection{Oscillating flap}

An oscillating flap was designed and implemented at the BFS to generate periodic small perturbations into the turbulent shear layer (Figure 2- 6). The flap was $370 \mathrm{~mm}$ long, $5 \mathrm{~mm}$ wide and $0.2 \mathrm{~mm}$ thick and was mounted like a ribbon at a height of $Y_{n}=5 \mathrm{~mm}$ over the step and parallel with the separation edge, as shown in Figure 2- 7. It was made of brass which is electro-conductive and diamagnetic. A spanwise row of Neodymium magnets were fixed under the surface of the step in the same orientation in order to produce an approximate parallel magnetic field around the oscillating flap. The oscillating flap was connected with an Agilent waveform generator, a Dynacord CL1600 power amplifier and a resistance of $5 \Omega$ in a series circuit. The alternating current and the perpendicular magnetic field generated vertical periodic Ampere force on the flap. If the oscillation amplitude is small, the fundamental frequency of standing waves in the flap depends on its length, linear density and tension. In the present case, the perturbation frequency was equal to the fundamental frequency of the standing wave, resulting in periodic small perturbations in the vertical direction. So the displacement of the center part of the flap can be described as: 


$$
Y_{\text {flap }}=A \cdot \cos \left(2 \pi \cdot f_{p} \cdot t\right)+Y_{n}
$$

The neutral position was $Y_{n}=5 \mathrm{~mm}$ over the step. The peak amplitude was $\mathrm{A}=2.5 \mathrm{~mm}$ as the maximum displacement from the neutral position. Then, the oscillating velocity of the center part of the flap was calculated by the time derivative of the displacement

$$
\mathrm{V}_{\text {flap }}=\mathrm{dY}_{\text {flap }} / \mathrm{dt}
$$

which enables to estimate the oscillating velocity to be in the range of $\max \left(\left|\mathrm{V}_{\text {flap }}\right|\right) \approx 0.86 \mathrm{~m} / \mathrm{s}$. The ratio of the oscillating displacement to the step height was $|\mathrm{A} / \mathrm{h}|<0.09$ and the ratio of the oscillating velocity to the free-stream velocity was $\left|V_{\text {flap }} / U_{0}\right|<0.09$, which therefore ensured the perturbations were sufficiently small compared with the BFS geometry and free-stream flow velocity.

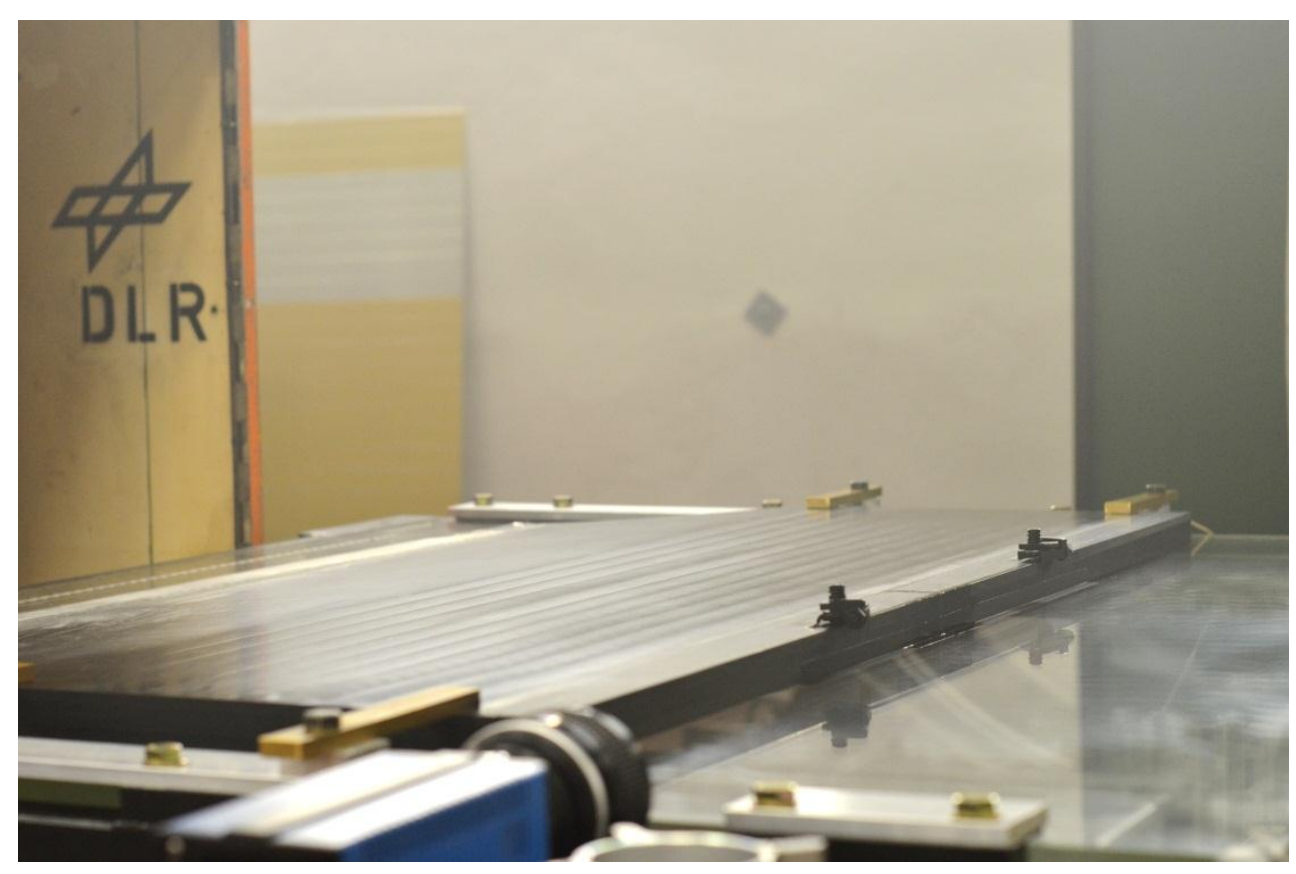

Figure 2- 6 Photograph of the oscillating flap on the backward-facing step 


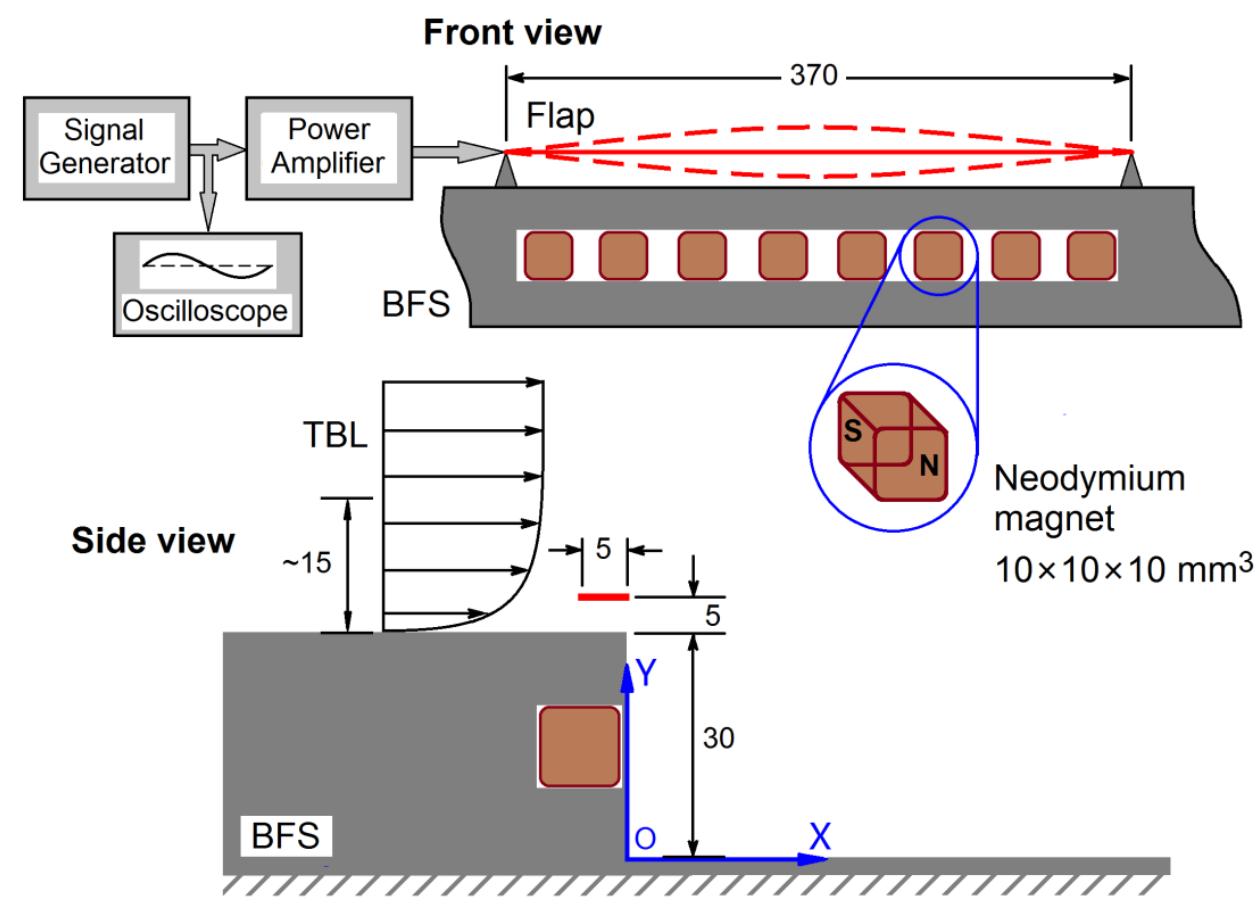

Figure 2- 7 Schematic diagram of oscillating flap in backward-facing step flow

The phase angle in one period was defined by the position of the oscillating flap between $\alpha=0^{\circ}$ and $360^{\circ}$. Thereby, phase angles $\alpha=0^{\circ}$ and $\alpha=180^{\circ}$ indicate the highest and lowest positions, respectively. The perturbation frequency was fixed at $f_{p}=55 \mathrm{~Hz}\left(\mathrm{St}_{\mathrm{h}}=0.165\right)$. The frequency spectrum near the center portion of the oscillating flap indicates that the perturbations contain the fundamental frequency $f_{p}$ as well as the overtones with lower amplitudes (Figure 2- 8). Previous experimental results show that the most-amplified frequency of the turbulent shear layer was at $\mathrm{f}_{0} \approx 100-120 \mathrm{~Hz}$ (Ma et al. 2014; Ma et al. 2015). Therefore, in the present study, the perturbation frequency was chosen to be only half of the fundamental frequency $f_{p} \approx f_{0} / 2$, which generated subharmonic waves into the turbulent shear layer downstream of the BFS. 


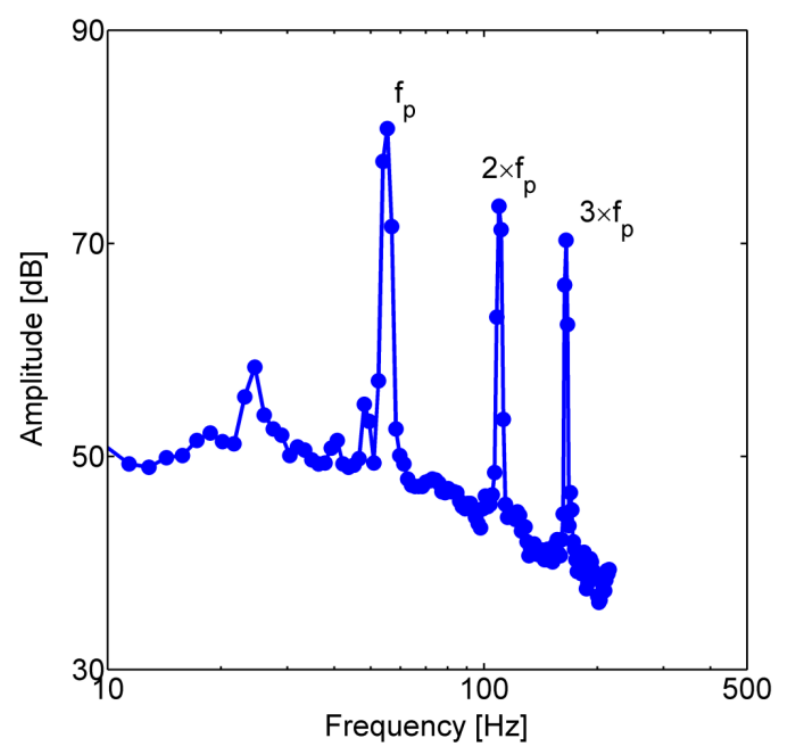

Figure 2- 8 Frequency spectrum of the oscillating flap at $\mathrm{f}_{\mathrm{p}}=55 \mathrm{~Hz}$

\subsection{Vortex generators}

In the present research, a spanwise-distributed array of low-profile wedge-type vortex generators were fixed on the BFS step (Figure 2- 9), each of which had a set of parameters including a non-dimensional height $\mathrm{H} / \delta$, a non-dimensional length $\mathrm{e} / \mathrm{H}$, an angle of incidence $\beta$, a non-dimensional spanwise spacing $\Delta \mathrm{Y} / \mathrm{H}$, a non-dimensional streamwise distance between trailing edges and the step $\Delta \mathrm{X} / \mathrm{H}$, and an orientation (Ashill et al. 2002). The first three parameters describe the device geometry, and the following three present the location. Among all the parameters, two parameters were tested: the VG heights of $\mathrm{H}=5$ and $10 \mathrm{~mm}$, and the orientations of backward and forward types, while the other parameters were kept constant as $\mathrm{e} / \mathrm{H}=10, \beta=14^{\circ}, \Delta \mathrm{Y} / \mathrm{H}=6$ and $\Delta \mathrm{X} / \mathrm{H}=10$. Combinations of the parameter sets resulted in four different VG configurations: "VG-H10b", "VG-H05b", "VG-H10f" and "VG-H05f". Each VG configuration was tested independently and the resulting flow field was measured by high-resolution 2D-2C PIV and time-resolved tomographic PIV. The clean BFS without VG was also tested for comparison. The detailed geometry of "VG-H10b" is given in Figure 2- 10. 


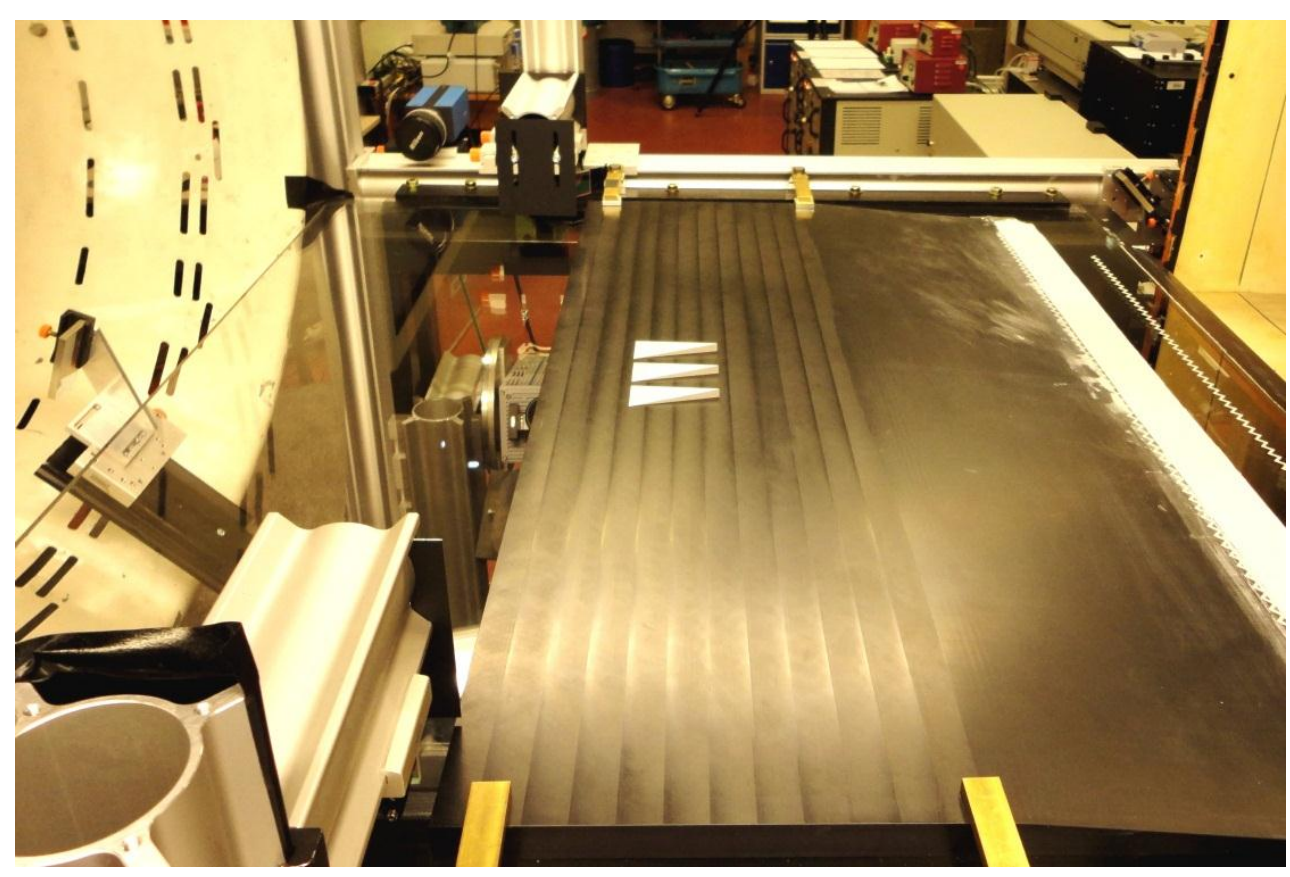

Figure 2- 9 Photograph of the "VG-H10b" configuration on the BFS
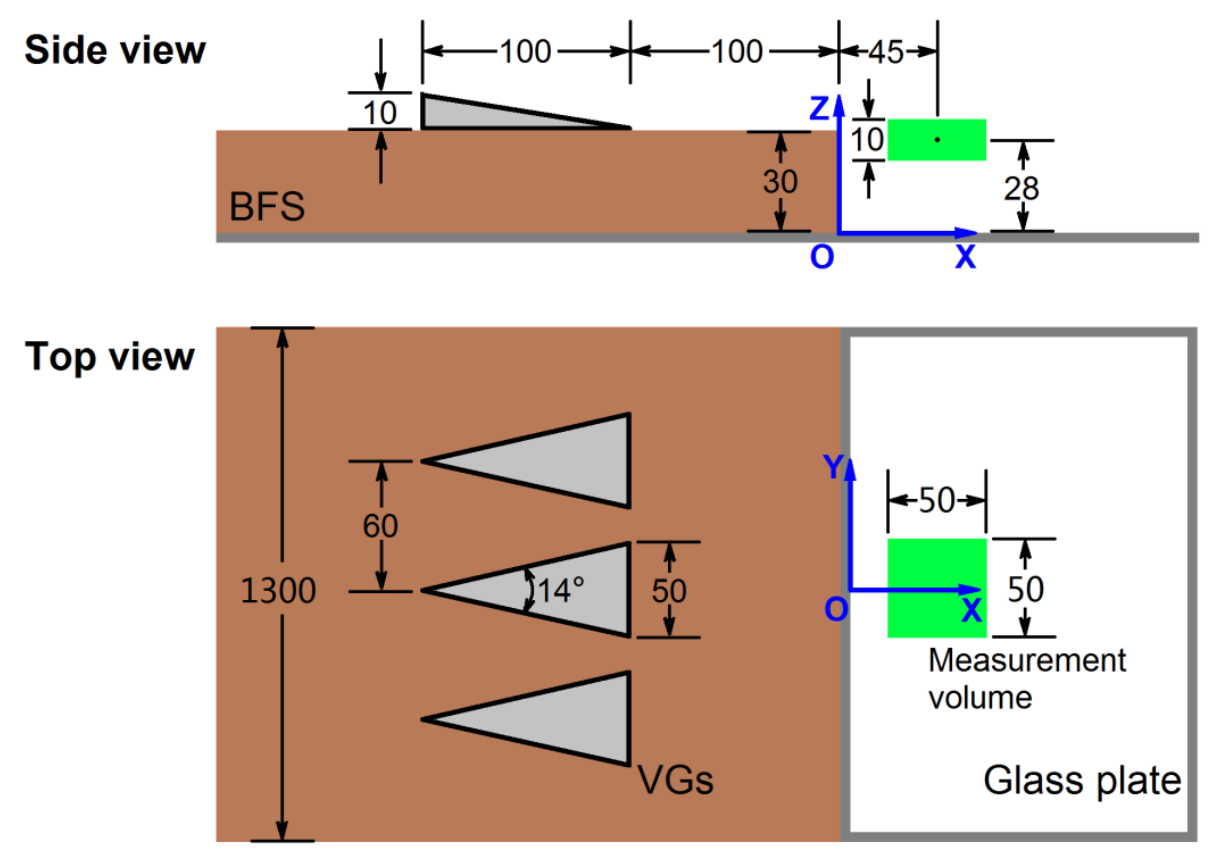

Figure 2- 10 Schematic diagram of the BFS geometry and "VG-H10b" configuration

\subsection{D-2C planar PIV}

Digital PIV systems have been widely used in fluid measurements for about two decades 
(Willert et al. 1991; Adrian 2005; Raffel et al. 2007). With the development of high-resolution cameras, 2D-2C planar PIV technique has been able to capture a large range of scales in fluid motions. In the present study, the turbulent BFS flow contains multi-scale fluid motions including not only the large-scale flow separation and reattachment but also the small-scale turbulent random fluctuations. A high-resolution 2D-2C PIV system has been used to measure the turbulent boundary layer, separated shear layer and reattachment area in a single field of view. The incoming flow was homogeneously seeded by DEHS droplets with a mean diameter of $1 \mu \mathrm{m}$ (Kähler et al. 2002). A single field of view of $310 \times 70 \mathrm{~mm}^{2}$ was illuminated by a laser light sheet in the streamwise-vertical direction with an approximate thickness of $1 \mathrm{~mm}$ from downstream orientation. The two laser pulses, generated by a Big Sky Ultra CFR Nd:YAG laser system, delivered an energy of $30 \mathrm{~mJ}$ per pulse with $150 \mu$ s time delay. Particle images were recorded by a high-resolution CCD camera PCO.4000 equipped with a Nikon lens (85mm, f/4) from a side view outside of the flow. The optical axis of the camera was perpendicular to the laser light sheet. Each image consisted of 3,900×910 pixels in X- and Y- directions, respectively. A constant magnification factor of $13.04 \mathrm{pixel} / \mathrm{mm}$ between the image and physical coordinates was obtained by a PIV calibration process. Synchronization of the image recording and illumination was accomplished by TTL signals from a programmable sequencer. Two steps were carried out in the recording procedure. First, 4,000 non-phase-locked double-frame images were recorded for the flows with and without flow control devices in order to calculate time-averages and corresponding root-mean-square values of the velocity vector fields. Second, 1,000 phase-locked double-frame images at 12 phases in one period of the perturbations introduced by the flow control devices were recorded in order to capture periodic motions in the turbulent shear layer. Image evaluation was performed by using PIVview software from PIVTEC. As a pre-process procedure, a minimum background image was subtracted from the particle images in order to reduce noise and eliminate local light inhomogeneity. Then, the double-frame images were evaluated by an iterative multi-grid cross-correlation method (Willert et al. 1991) with image deformation and a final interrogation window size of $16 \times 16$ pixel at $75 \%$ overlap, resulting in a vector spacing of $0.3 \mathrm{~mm}(=0.01 \cdot \mathrm{h})$ in the physical coordinate. The $2 \mathrm{D}-2 \mathrm{C}$ PIV 
systems for the measurements of the acoustic tube, oscillating flap and vortex generators are shown in Figure 2- 11, Figure 2- 12 and Figure 2- 13, which have the same configuration and field of view. A two-dimensional coordinate system has its origin point at the corner on the wall. The $\mathrm{X}$ - and Y-axis correspond to the streamwise and vertical directions, respectively. The corresponding velocity components are denoted as $\mathrm{u}$ and $\mathrm{v}$.

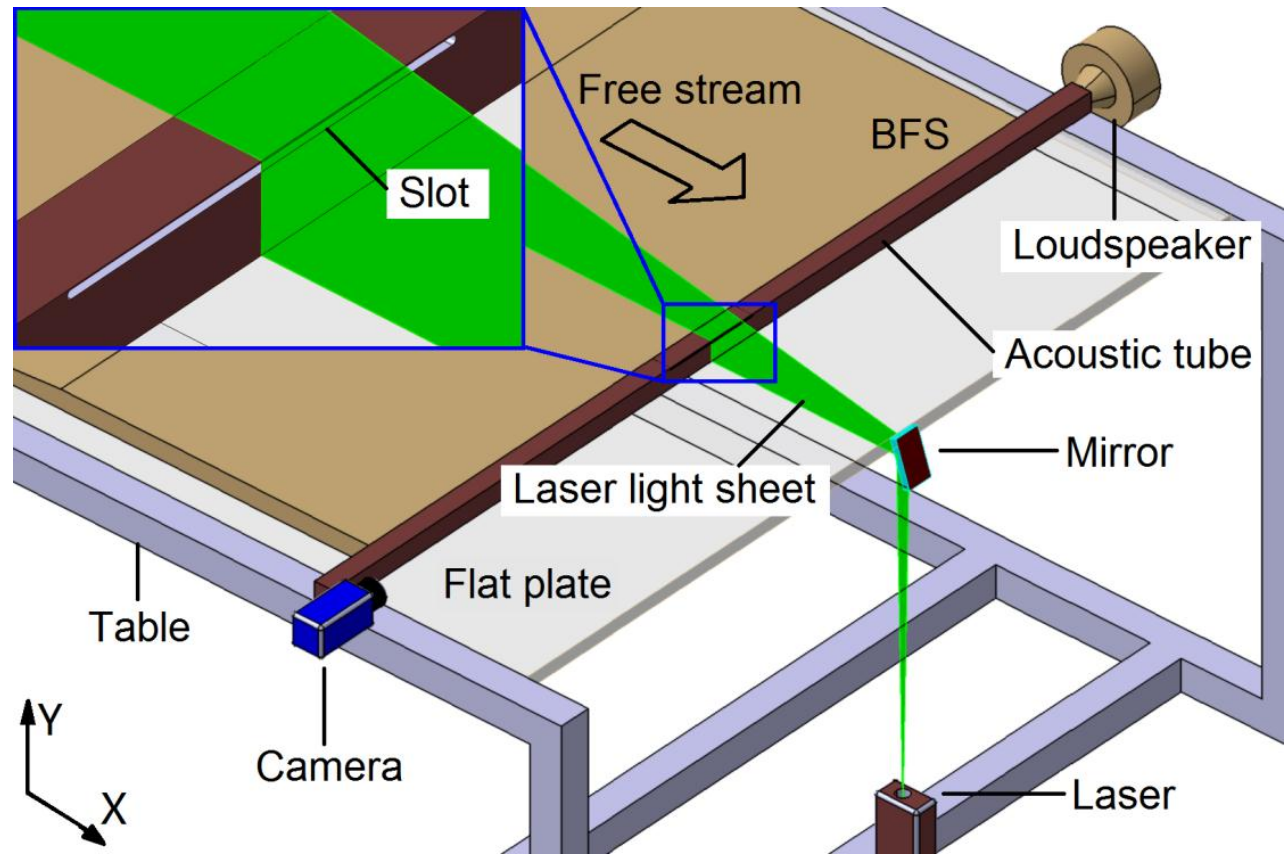

Figure 2- 11 Schematic diagram of 2D-2C PIV system and close-up view of the acoustic tube

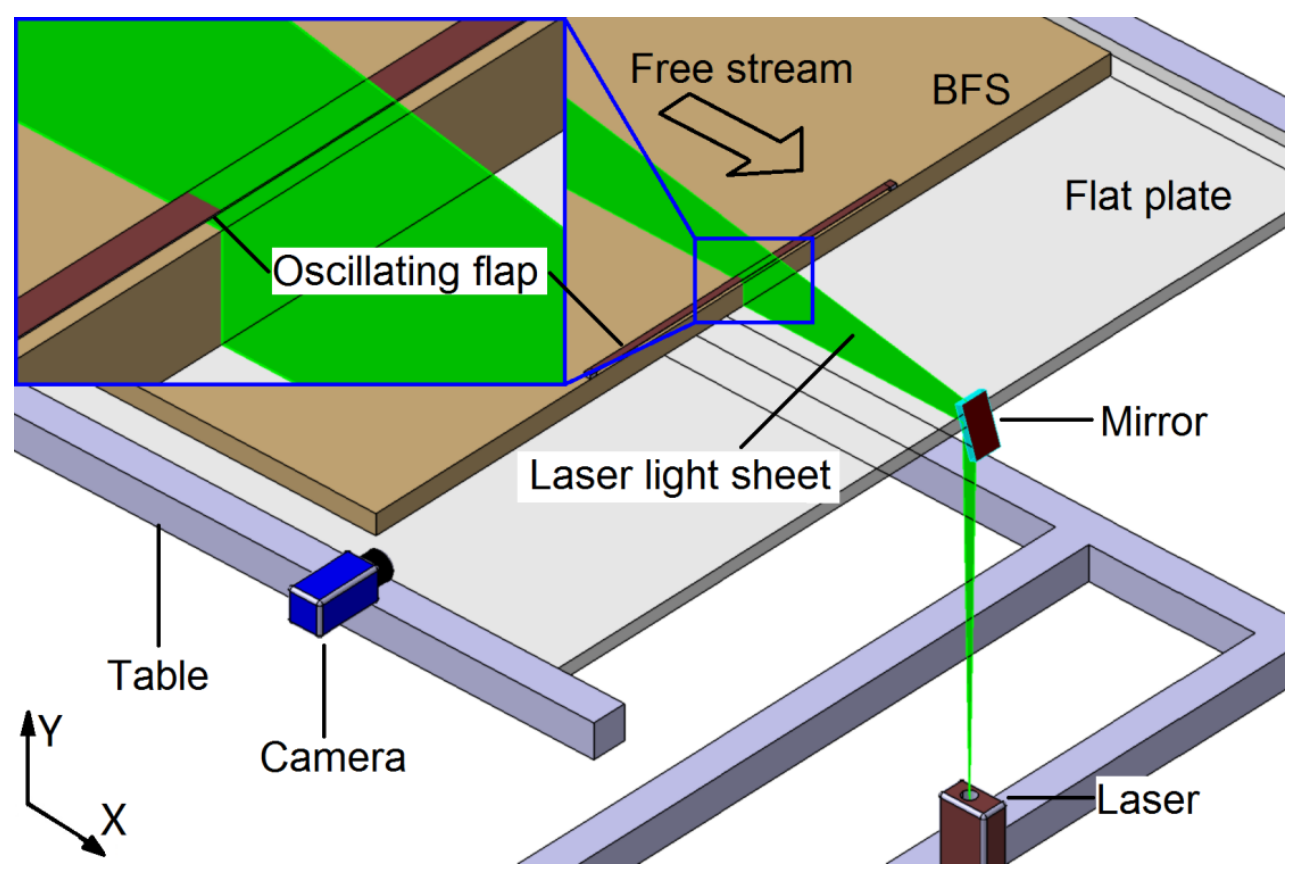

Figure 2- 12 Schematic diagram of 2D-2C PIV system and close-up view of the oscillating flap 


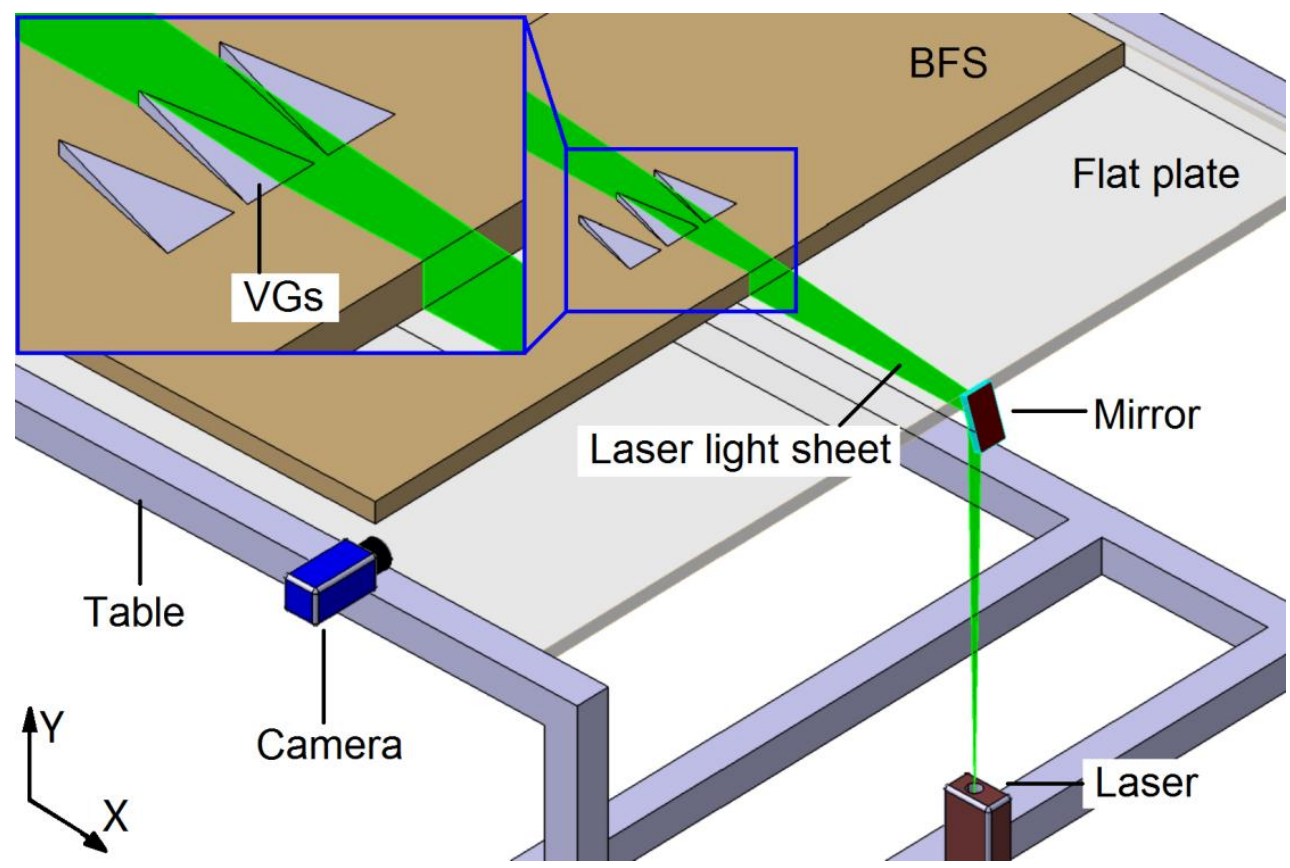

Figure 2- 13 Schematic diagram of 2D-2C PIV system and close-up view of the vortex generators

\subsection{D-3C(t) time-resolved tomographic PIV}

Time-resolved tomographic PIV was used to measure the turbulent shear layer downstream of the BFS (Figure 2- 14). Four Photron APX-RS high-speed CMOS cameras $(1024 \times 1024$ pixels, 10 bits) equipped with Nikon lenses $(105 \mathrm{~mm}, \mathrm{f} / 8)$ were mounted in a pyramidal configuration under the glass plate, all of which had the same field of view. The three-dimensional measurement volume had a size of $50 \times 50 \times 10 \mathrm{~mm}^{3}$ in $\mathrm{X}-$, Y- and Z-direction, respectively, which covered a streamwise area from $12 \cdot \mathrm{H}$ to $17 \cdot \mathrm{H}$ downstream of the VGs. The center of the volume was located $45 \mathrm{~mm}$ behind the step and $28 \mathrm{~mm}$ over the plate. A laser light beam was generated by a high-resolution Nd:YAG laser system from LEE inc. and was aligned parallel with the step in the spanwise direction, and then was reflected back between two mirrors on both sides in order to increase the energy within the volume and generate homogenous light scattering of the particles for all camera viewings. The double laser pulses delivered energy of $17 \mathrm{~mJ}$ per pulse with a time delay of $60 \mu \mathrm{s}$. A sequence of 3,000 double-frame particle images was recorded at a sampling rate of $1 \mathrm{kHz}$ in frame-straddling mode for both the clean and controlled cases. 


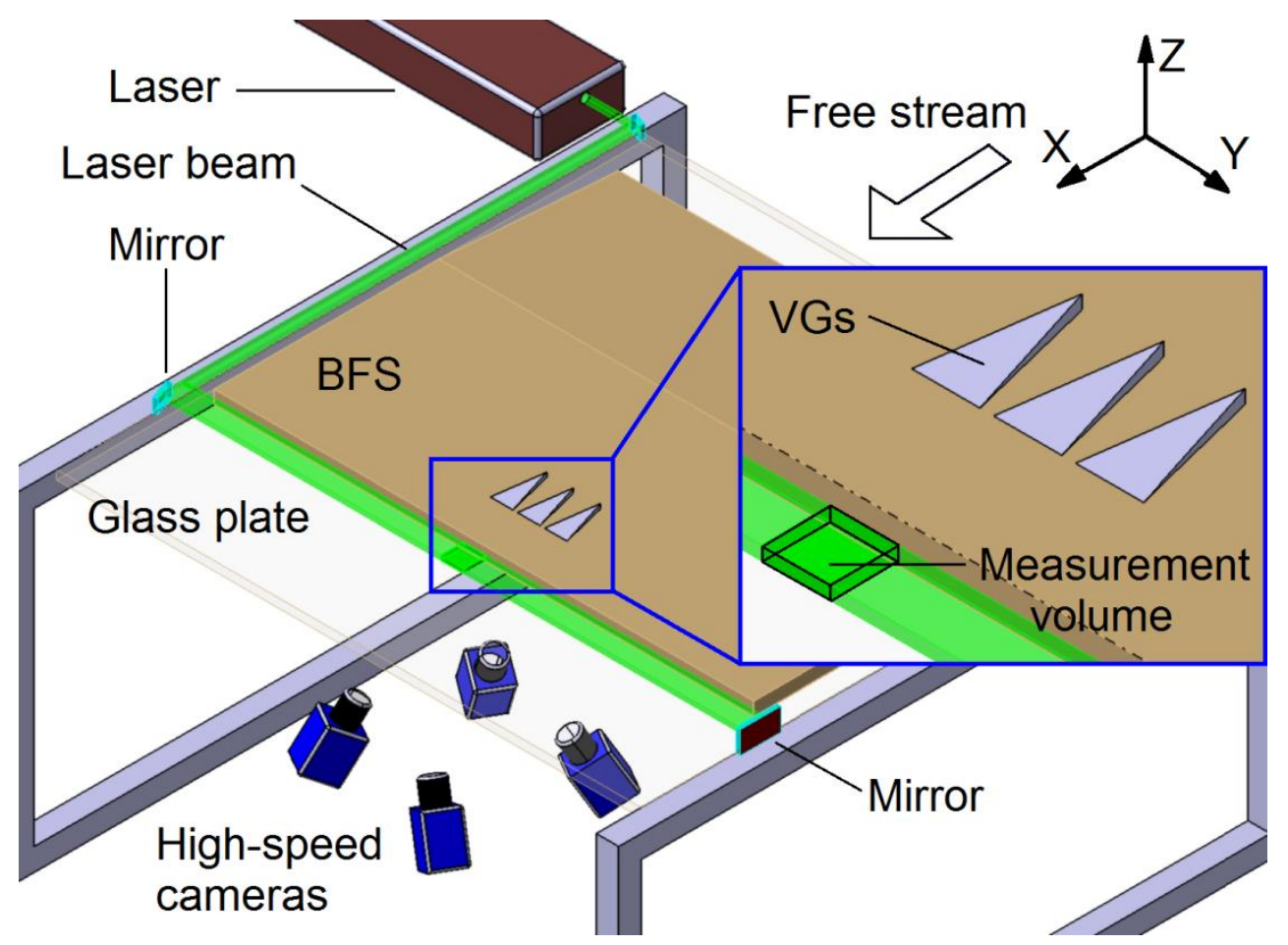

Figure 2- 14 Schematic diagram of tomographic PIV system and close-up view on the VGs and measurement volume

Particle image acquisition has been realized by Photron camera software and particle image evaluation was performed by a DLR own SMART algorithm for tomographic volume reconstruction and a 3D cross-correlation scheme of DaVis7.3. First, a mapping function between the image planes and physical space was obtained by a 3D calibration procedure using a precision-machined dual level calibration target. Second, sparse particle image distributions of the four simultaneous camera images were used for volume-self-calibration in order to enhance the accuracy of the reconstruction step (Wieneke 2008) and then an array of $865 \times 996 \times 228$ voxels were reconstructed by means of SMART including the determination and application of the optical transfer function as weights (Schanz et al. 2013). Third, the particle image volume was analyzed by direct three-dimensional direct cross-correlation with an iterative multi-grid volume deformation scheme. Finally, with a final $48^{3}$ voxels interrogation box size at $75 \%$ overlap an instantaneous three-dimensional velocity vector field was achieved which consisted of $60 \times 60 \times 14$ measurement points with a vector spacing of $0.752 \mathrm{~mm}(\approx 0.025 \cdot \mathrm{h})$. A 
three-dimensional coordinate system has its origin point at the corner behind of the step. The X-, Y- and Z-axis correspond to the streamwise, spanwise and vertical directions, respectively. The velocity components are denoted as $\mathrm{u}, \mathrm{v}$ and $\mathrm{w}$. (The vertical direction of the $3 \mathrm{D}$ measurement is different from the 2D measurement.) The measurement parameters are listed in Table 2- 1.

Table 2- 1 Parameters of 2D-2C and tomographic PIV systems

\begin{tabular}{lll}
\hline \hline & High-resolution 2D-2C PIV & Time-resolved tomographic PIV \\
\hline Recording medium & PCO.4000 camera & Photron APX-RS camera \\
Recording method & double frame / single exposure & double frame / single exposure \\
Resolution & $4,000 \times 1,000$ pixels & $1,024 \times 1,024$ pixels \\
Dynamic range & 14 bits & 10 bits \\
Lens & Nikon $\mathrm{f}=85, \mathrm{f} / 4$ & Nikon micro $\mathrm{f}=105 \mathrm{~mm}, \mathrm{f} / 8$ \\
Observation distance & approx. $800 \mathrm{~mm}$ & approx. $400 \mathrm{~mm}$ \\
Field of view & $310 \times 70 \mathrm{~mm}{ }^{2}$ & $50 \times 60 \times 10 \mathrm{~mm}{ }^{3}$ \\
Sampling frequency & $1.4 \mathrm{~Hz}$ & $1 \mathrm{kHz}$ \\
Laser & Nd:YAG laser with $30 \mathrm{~mJ} / \mathrm{pulse}$ & Nd:YAG laser with $17 \mathrm{~mJ} / \mathrm{pulse}$ \\
Pulse delay & $150 \mu \mathrm{s}$ & $60 \mu \mathrm{s}$ \\
Seeding material & DEHS particles of $\mathrm{d}_{\mathrm{p}} \approx 1.0 \mu \mathrm{m}$ & DEHS particles of $\mathrm{d}_{\mathrm{p}} \approx 1.0 \mu \mathrm{m}$ \\
& & MLOS-SMART reconstruction \\
Final interrogation size & $16 \times 16$ pixels & algorithm and multi-grid \\
Overlap & $75 \%$ & interrogation method \\
Vector spacing & $0.25-0.3 \mathrm{~mm}$ & $128 \times 128 \times 128$ voxels \\
\hline \hline
\end{tabular}

"Vortices are the sinews and muscles of fluid motions."

Dietrich Küchemann (1918-1988) 


\section{3 Post-processing methods}

\subsection{Reynolds shear stress}

The phase-locked measurements provide insight into the development of quasi-periodic spanwise vortices in the turbulent shear layer. By applying the triple decomposition (Hussain and Reynolds 1970), an instantaneous velocity vector can be decomposed as:

$$
\mathbf{u}=\overline{\mathbf{u}}+\widetilde{\mathbf{u}}+\mathbf{u}^{\prime}
$$

The term on the left-hand side is an instantaneous velocity vector. The terms on the right-hand side are mean, periodic and random fluctuating parts, which correspond to the time-averaged flow, periodic coherent structures and incoherent turbulence, respectively. The periodic part can be obtained by subtracting the mean flow from the phase-averaged flows.

$$
\widetilde{\mathbf{u}}=\langle\mathbf{u}\rangle-\overline{\mathbf{u}}
$$

As an essential quantity in turbulent flows, Reynolds shear stress represents the quantity of the fluid particles fluctuating upwards and downwards between the shear layers (Schlichting 1979). Additionally, the periodic components are uncorrelated with the random fluctuations:

$$
\overline{\tilde{\mathrm{u}} \cdot \mathrm{v}^{\prime}}=0, \quad \overline{\mathrm{u}^{\prime} \cdot \overline{\mathrm{v}}}=0
$$

Therefore, the Reynolds shear stress can be decomposed as: 


$$
\tau_{\mathrm{xy}}^{\prime}=-\rho \cdot \overline{\left(\tilde{\mathrm{u}}+\mathrm{u}^{\prime}\right) \cdot\left(\tilde{\mathrm{v}}+\mathrm{v}^{\prime}\right)}=-\rho \cdot\left(\overline{\tilde{\mathrm{u}} \cdot \tilde{\mathrm{v}}}+\overline{\mathrm{u}^{\prime} \cdot \mathrm{v}^{\prime}}\right)
$$

The terms $\overline{\tilde{u} \cdot \tilde{v}}$ and $\overline{u^{\prime} \cdot v^{\prime}}$ indicate to the contributions of the coherent motions and incoherent turbulence, respectively. Furthermore, the incoherent Reynolds shear stress $\left\langle u^{\prime} \cdot v^{\prime}\right\rangle$ at different phases are analyzed by the quadrant analysis. Four quadrant events of the random fluctuating velocities $u^{\prime}$ and $v^{\prime}$ are defined as (Wallace et al. 1972; Willmarth and Lu 1972):

$$
\left\langle u^{\prime} \cdot v^{\prime}\right\rangle=\sum_{i=1}^{4}\left\langle Q_{i}\right\rangle, \quad i= \begin{cases}1, & u^{\prime}>0, v^{\prime}>0 \\ 2, & u^{\prime}<0, v^{\prime}>0 \\ 3, & u^{\prime}<0, v^{\prime}<0 \\ 4, & u^{\prime}>0, v^{\prime}<0\end{cases}
$$

The quadrant analysis reveals the influences of the small perturbations on the events of Reynolds shear stress, while the quadrants $\mathrm{Q}_{2}$ and $\mathrm{Q}_{4}$ are events of turbulence production.

\subsection{Two-point cross-correlation function}

The spatial and temporal cross-correlation functions, first introduced by Taylor (1935, 1936), have been frequently used to quantitatively depict characteristic structures and estimate convection velocities in a turbulent boundary layer (Favre et al. 1957), turbulent shear layer (Wills 1964), pipe flow (McConachie 1981) and cavity flow (Bian et al. 2011). A coefficient for two-point spatial cross-correlation with a reference point $\overrightarrow{\mathrm{r}_{0}}=\left(\mathrm{X}_{0}, \mathrm{Y}_{0}, \mathrm{Z}_{0}\right)$ in the same time duration is defined as:

$$
\mathrm{R}_{\mathrm{u}_{\mathrm{i}}^{\prime} \mathrm{u}_{\mathrm{j}}^{\prime}}(\Delta \overrightarrow{\mathrm{r}})=\frac{\overline{\mathrm{u}_{1}{ }^{\prime}\left(\overrightarrow{\mathrm{r}_{0}}, \mathrm{t}\right) \cdot \mathrm{u}_{\mathrm{j}}{ }^{\prime}\left(\overrightarrow{\mathrm{r}_{0}}+\Delta \overrightarrow{\mathrm{r}}, \mathrm{t}\right)}}{\sqrt{\overline{\mathrm{u}_{1}{ }^{\prime 2}\left(\overrightarrow{\mathrm{r}_{0}}, \mathrm{t}\right)}} \cdot \sqrt{\overline{\mathrm{u}_{\mathrm{j}}{ }^{\prime 2}\left(\overrightarrow{\mathrm{r}_{0}}+\Delta \overrightarrow{\mathrm{r}}, \mathrm{t}\right)}}}
$$

here $\Delta \vec{r}=(\Delta x, \Delta y, \Delta z)$ is the displacement vector from the reference point and $\mathrm{u}_{\mathrm{i}}^{\prime}, \mathrm{u}_{\mathrm{j}}^{\prime}$ are the fluctuating velocity components.

Moreover, the coefficient for two-point temporal cross-correlation with a reference point $\overrightarrow{\mathrm{r}_{0}}=\left(\mathrm{X}_{0}, \mathrm{Y}_{0}, \mathrm{Z}_{0}\right)$ and a time step $\mathrm{dt}$ is defined as: 


$$
\mathrm{R}_{\mathrm{u}_{\mathrm{i}}^{\prime} \mathrm{u}_{\mathrm{j}}^{\prime}}(\Delta \overrightarrow{\mathrm{r}}, \mathrm{dt})=\frac{\overline{\mathrm{u}_{1}^{\prime}\left(\overrightarrow{\mathrm{r}_{0}}, \mathrm{t}\right)} \cdot \mathrm{u}_{\mathrm{j}}{ }^{\prime}\left(\overrightarrow{\mathrm{r}_{0}}+\Delta \overrightarrow{\mathrm{r}}, \mathrm{t}+\mathrm{dt}\right)}{\sqrt{\overline{\mathrm{u}_{1}^{\prime}{ }^{\prime 2}\left(\overrightarrow{\mathrm{r}_{0}}, \mathrm{t}\right)}} \cdot \sqrt{\overline{\mathrm{u}_{\mathrm{j}}{ }^{\prime 2}\left(\overrightarrow{\mathrm{r}_{0}}+\Delta \overrightarrow{\mathrm{r}}, \mathrm{t}+\mathrm{dt}\right)}}}
$$

where $\mathrm{dt}$ is the time step between two snapshots in the present investigation using time-resolved tomographic PIV.

\subsection{Proper orthogonal decomposition}

Although coherent structures play a predominant role in turbulent shear flows, they are easy to recognize by flow visualization but difficult to understand completely because a wide scale range of vortices are superimposed on random fluctuations, especially at high Reynolds numbers. Nowadays, $2 \mathrm{D}-2 \mathrm{C}$ particle image velocimetry is able to capture a large range of scales in fluid motions due to the use of high-resolution cameras. However, an increasing amount of experimental data requires more ensemble and efficient methods to analyze essential flow structures and corresponding dynamic features, and then eventually lead to a better understanding of underlying physical mechanisms. Proper orthogonal decomposition (POD), firstly introduced by Lumley (1967), enables to linearly decompose ensembles of data to a series of spatially orthogonal modes, each of which is hierarchically ranked by turbulent kinetic energy (Berkooz et al. 1993). As a special case of the well-known singular value decomposition (SVD), snapshot POD (Sirovich 1987) is a popular method to characterize coherent structures in turbulence, which has been applied to various transitional and turbulent flows including wall-bounded flows (Pedersen et al. 2002; Gurka et al. 2006; Ma et al. 2015), turbulent wakes (Bonnet et al. 1994; Santa Cruz et al. 2005), and turbulent jets (Bernero et al. 2000; Perrin et al. 2007; Meyer et al. 2007).

In order to reveal the spatial structures of the vortices, the snapshot POD method is applied in a rectangular region, which covers the shear layer downstream of the step. The mathematical background is explicitly presented in Meyer et al. (2007). First, all the snapshots containing the fluctuating velocity components are arranged in a matrix $U$ as: 


$$
\mathbf{U}=\left[\begin{array}{llll}
\mathbf{u}_{1} & \mathbf{u}_{2} & \cdots & \mathbf{u}_{\mathrm{N}}
\end{array}\right]
$$

Each column corresponds to one PIV snapshot. Second, the eigenvalues of the auto-covariance matrix are calculated as:

$$
\tilde{\mathbf{C}} \cdot \mathbf{A}_{\mathbf{i}}=\lambda_{\mathbf{i}} \cdot \mathbf{A}_{\mathbf{i}} \text {, with } \tilde{\mathbf{C}}=\mathbf{U}^{\mathbf{T}} \cdot \mathbf{U}
$$

and then are ranked in a descending order as:

$$
\lambda_{1}>\lambda_{2}>\cdots>\lambda_{N}=0
$$

Third, each mode is obtained by projecting matrix $U$ onto each eigenvector and is then normalized by its Euclidean norm:

$$
\operatorname{POD}_{i} \equiv \phi_{i}=\frac{\sum_{n=1}^{N}\left(\mathbf{A}_{\mathbf{i}}^{\mathbf{n}} \cdot \mathbf{u}_{\mathbf{n}}\right)}{\left\|\sum_{n=1}^{\mathrm{N}}\left(\mathbf{A}_{\mathbf{i}}^{\mathbf{n}} \cdot \mathbf{u}_{\mathbf{n}}\right)\right\|}
$$

so

$$
\Phi=\left[\begin{array}{llll}
\phi_{1} & \phi_{2} & \cdots & \phi_{\mathrm{N}}
\end{array}\right]
$$

Coefficients of each POD mode can be obtained by projecting the original fluctuating velocity field onto each POD mode:

$$
\mathbf{a}_{\mathbf{n}}=\boldsymbol{\Phi}^{\mathrm{T}} \cdot \mathbf{u}_{\mathbf{n}}
$$

Therefore, POD decomposes the original velocity data sequence into the mean flow and the linear combination of spatially orthogonal modes as:

$$
\mathbf{U}=\overline{\mathbf{U}}+\sum_{\mathrm{i}=1}^{\mathrm{N}} \mathbf{a}_{\mathbf{i}} \cdot \boldsymbol{\phi}_{\mathbf{i}}
$$

$\Phi_{\mathrm{i}}$ is the i-th mode and ai is the corresponding coefficient. All the modes are ranked based on the descending order of the eigenvalues, which represent the turbulent kinetic energy (Meyer et al. 2007). This energy-based hierarchy ensures that the predominant modes containing higher energy are represented in the first few modes, which may correspond to the large-scale coherent structures, whereas all further modes containing lower energy are small-scale or random 
fluctuations of less importance.

\subsection{Dynamic mode decomposition}

Dynamic mode decomposition (DMD) is able to extract dynamic features by linearly decomposing flow fields into a series of single-frequency/wavenumber dynamic modes (Schmid 2010). Being different from the POD method, DMD results in a series of temporally orthogonal modes, each of which has a single frequency and a temporal decay. As a recent post-processing method, DMD has gained considerable popularity and has been applied to identify coherent motions and corresponding dynamic features such as the wake of a Gurney flap (Pan et al. 2011), vortex rings of a transitional jet (Schmid et al. 2012) and hairpin vortices in a turbulent boundary layer (Tang et al. 2012).

Dynamic mode decomposition (DMD) is another matrix-based method which enables to extract single-frequency/single-wavenumber dynamic modes by applying the constraint condition of temporal orthogonality to the matrix decomposition (Schmid 2010). In order to focus on large-scale flow structures and avoid the influence of incoherent turbulence, in the present study, the DMD method is applied to the POD reconstructed data which only contain predominant POD modes and have filtered out higher modes. The "evolving direction" can be a temporal sequence or a spatial coordinate, which is then denoted as the "spatial DMD method". The mathematical algorithm is briefly presented. First, the velocity components are arranged in two matrices $\mathbf{V}_{\mathbf{x}}$ and $\mathbf{V}_{\mathbf{x}+\Delta \mathbf{x}}$ :

$$
\begin{aligned}
& \mathbf{V}_{\mathbf{x}}=\left[\begin{array}{llll}
\mathbf{v}_{1} & \mathbf{v}_{2} & \cdots & \mathbf{v}_{\mathbf{X}-\mathbf{1}}
\end{array}\right] \\
& \mathbf{V}_{\mathbf{x}+\Delta \mathbf{x}}=\left[\begin{array}{llll}
\mathbf{v}_{\mathbf{2}} & \mathbf{v}_{\mathbf{3}} & \cdots & \mathbf{v}_{\mathbf{X}}
\end{array}\right]
\end{aligned}
$$

Both of $\mathbf{V}_{\mathbf{x}}$ and $\mathbf{V}_{\mathbf{x}+\Delta \mathbf{x}}$ consist of X-1 columns of velocity data in the streamwise direction with the constant distance of $\Delta \mathrm{x}$. Then it is assumed that a linear mapping matrix $\mathbf{P}$ propagates each snapshot $\mathrm{v}_{\mathrm{x}}$ to $\mathrm{v}_{\mathrm{x}+1}$ over the distance of $\Delta \mathrm{x}$. 


$$
\mathbf{P} \cdot \mathbf{V}_{\mathbf{x}}=\mathbf{V}_{\mathbf{x}+\Delta \mathbf{x}}
$$

Theoretically, this propagation matrix $\mathbf{P}$ contains all the information of the turbulent flow. However, the matrix $\mathbf{P}$ is of too high dimensionality to be solved directly. So a similar but less dimensional matrix $\mathbf{S}$, which approximates the underlying propagation, is introduced to replace the unknown matrix $\mathbf{P}$. By SVD, the original data sequence can be decomposed into a basis $\mathbf{U}_{\text {svd }}$, a diagonal matrix $\boldsymbol{\Sigma}_{\text {svd }}$ and another basis $\mathbf{V}_{\text {svd }}$ :

$$
\mathbf{V}_{\mathbf{x}}=\mathbf{U}_{\mathrm{svd}} \cdot \mathbf{\Sigma}_{\mathrm{svd}} \cdot \mathbf{V}_{\mathrm{svd}}^{\mathrm{H}}
$$

The resulting X-1 singular values are contained in a diagonal matrix $\boldsymbol{\Sigma}_{\text {svd: }}$ :

$$
\boldsymbol{\Sigma}_{\text {svd }}=\left[\begin{array}{lll}
\mathrm{s}_{1} & & \\
& \ddots & \\
& & \mathrm{s}_{\mathrm{X}-1}
\end{array}\right]
$$

Therefore, the unknown matrix $\mathbf{P}$ is projected onto the orthogonal basis $\mathbf{U}_{\text {svd }}$. Matrix $\mathbf{S}$ is then derived as:

$$
\mathbf{S} \equiv \mathbf{U}^{\mathbf{H}} \cdot \mathbf{P} \cdot \mathbf{U}=\mathbf{U}^{\mathbf{H}} \cdot \mathbf{V}_{\mathbf{x}+\Delta \mathbf{x}} \cdot \mathbf{V} \cdot \mathbf{\Sigma}^{-\mathbf{1}}
$$

The resulting matrix $\mathbf{S}$ approximates the propagation matrix $\mathbf{P}$, but it has a much smaller size. In the next step, the eigenvalues and eigenvector of $\mathbf{S}$ are solved as:

$$
\mathbf{S} \cdot \mathbf{E}_{\mathbf{i}}=\lambda_{\mathrm{i}} \cdot \mathbf{E}_{\mathbf{i}}
$$

Because each snapshot in the sequence is assumed to spatially evolve by $e^{2 \pi \cdot f_{i} \cdot \Delta x}$, complex frequencies can be derived as:

$$
\mathrm{f}_{\mathrm{i}}=\frac{\ln \left(\lambda_{\mathrm{i}}\right)}{2 \pi \cdot \Delta \mathrm{x}}
$$

The real part of the complex frequency indicates the spatial growth rate while the imaginary part indicates the wavenumber. Third, each mode, denoted by $\operatorname{DMD}_{\mathrm{i}}(\mathrm{i}=1,2, \ldots, \mathrm{X}-1)$, is calculated by projecting the basis $\mathbf{U}_{\text {svd }}$ onto each eigenvector $\mathbf{E}_{\mathbf{i}}$ :

$$
\mathrm{DMD}_{\mathrm{i}} \equiv \boldsymbol{\Psi}_{\mathbf{i}}=\mathbf{U}_{\mathbf{s v d}} \cdot \mathbf{E}_{\mathbf{i}}
$$


and then arranged as

$$
\Psi=\left[\begin{array}{llll}
\boldsymbol{\Psi}_{1} & \boldsymbol{\Psi}_{2} & \cdots & \boldsymbol{\Psi}_{\mathrm{X}-1}
\end{array}\right]
$$

Here a spatial-evolving matrix $\mathbf{F}$ is formed as:

$$
\mathbf{F}=\left[\begin{array}{cccc}
1 & \left(\lambda_{1}\right)^{1} & \cdots & \left(\lambda_{1}\right)^{\mathrm{X}-2} \\
\vdots & \vdots & \ddots & \vdots \\
1 & \left(\lambda_{\mathrm{N}-1}\right)^{1} & \cdots & \left(\lambda_{\mathrm{N}-1}\right)^{\mathrm{X}-2}
\end{array}\right]
$$

To recover the amplitudes of each mode, the inverse matrix $\mathbf{A}^{-1}$ is calculated as:

$$
\mathbf{A}^{-\mathbf{1}}=\mathbf{F} \cdot \mathbf{V} \cdot \boldsymbol{\Sigma}^{\mathbf{- 1}} \cdot \mathbf{E}=\left[\begin{array}{lll}
\frac{1}{\mathrm{amp}_{1}} & & \\
& \ddots & \\
& & \frac{1}{\mathrm{amp}_{\mathrm{X}-1}}
\end{array}\right]
$$

Finally, the flow field can be reconstructed by:

$$
\mathbf{V}_{\mathbf{x}}=\boldsymbol{\Psi} \cdot \mathbf{A} \cdot \mathbf{F}
$$

The spatial-evolving flow field can be reconstructed by multiplying the DMD mode with the corresponding amplitude and evolution. The absolute values of the amplitude $\left|\mathrm{amp}_{\mathrm{i}}\right|$ measure the importance of the DMD modes. High amplitude indicates great contribution to the flow field while lower one indicates less. The distribution of the amplitude versus the wavenumber shows the spectrum of the flow field.

"Big whirls have little whirls that feed on their velocity; and little whirls have lesser whirls and so on to viscosity."

Lewis Fry Richardson (1881-1953) 


\section{Results of acoustic tube}

The instantaneous velocity vector field provides a direct visualization of the turbulent backward-facing step flow. In a stationary reference frame, the boundary layer separates from the step at the point $(\mathrm{X} / \mathrm{h}=0.0, \mathrm{Y} / \mathrm{h}=1.0)$ and forms a turbulent shear layer behind the backward-facing step, as shown in Figure 4- 1(a). On the other hand, the instantaneous velocity vector can be represented by the Galilean decomposition as (Adrian et al. 2000):

$$
\mathbf{u}=\mathbf{u}_{\mathbf{c}}+\mathbf{U}_{\mathbf{c}}
$$

Therefore in a moving reference frame at a convection velocity of $\mathrm{U}_{\mathrm{c}}=0.38 \cdot \mathrm{U}_{0}(\mathrm{Ma}$ et al. 2014b), the vortices are identified from the turbulent shear layer, although they are neither clear nor regular due to the strong turbulent mixing motions in Figure 4- 1(b). The convection velocity of the vortices in the shear layer is obtained by two-point cross-correlations based on time-resolved tomographic PIV data. It is discussed in detailed in Chapter 6. 

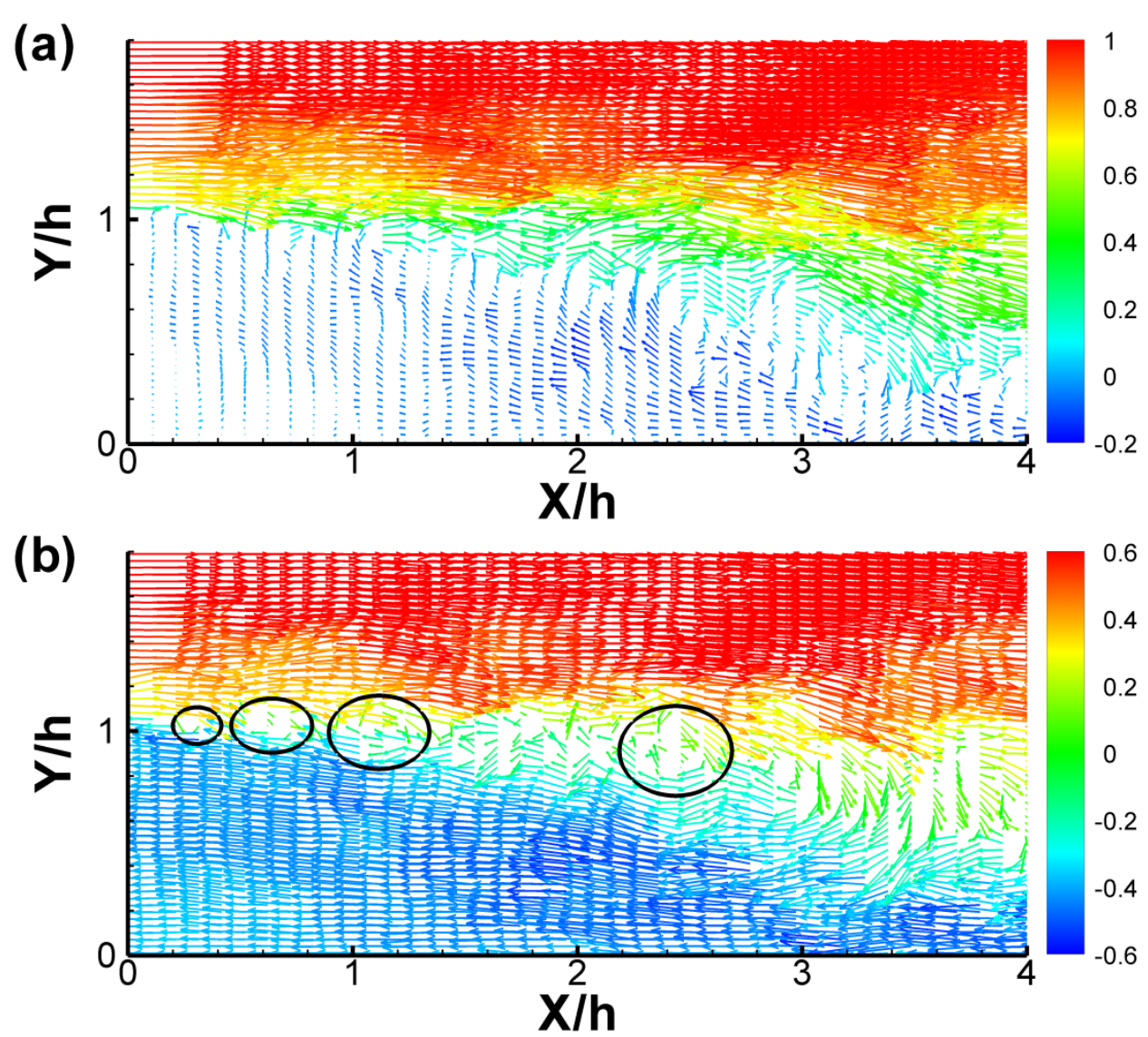

Figure 4- 1 Instantaneous velocity vector fields of the clean case of the backward-facing step flow. (a) in the stationary reference frame; (b) in the moving reference frame of $U_{c}=0.38 \cdot U_{0}$. The color indicates streamwise velocity component $\mathrm{u}_{\mathrm{c}} / \mathrm{U}_{0}$. Every 10 th vector in the streamwise direction and every 3rd vector in the vertical direction is shown for visual clarity.

\subsection{Convergence of mean and root-mean-square velocities}

In the 2D-2C PIV measurement, 4,000 statistically independent snapshots are obtained at a sampling frequency of $1.4 \mathrm{~Hz}$ in each case. The time-averaged and root-mean-square velocities of the clean case are shown in Figure 4- 2. In order to examine statistical convergence of the samples, five discrete points as well as a full rectangular field of view downstream of the backward-facing step are chosen as sample points. As shown in Figure 4- 3, the point No. 1 locates in the outer flow; the point No. 2 locates in the initial part of the shear layer; the point No. 3 locates in the recirculation region; the points No. 4 and No. 5 locate further downstream in the shear layer as well; the field of view has a size of $3.3 \mathrm{~h} \times 2.0 \mathrm{~h}$ and covers the rectangular 
region behind the step. The mean and r.m.s. velocities are calculated by:

$$
\begin{gathered}
\bar{u}=\frac{1}{N} \sum_{i=1}^{N} u_{i}, \bar{v}=\frac{1}{N} \sum_{i=1}^{N} v_{i} \\
u_{\text {r.m.s. }}=\sqrt{\frac{1}{N-1} \sum_{i=1}^{N}\left(u_{i}-\bar{u}\right)^{2}, v_{\text {r.m.s. }}=\sqrt{\frac{1}{N-1} \sum_{i=1}^{N}\left(v_{i}-\bar{v}\right)^{2}}}
\end{gathered}
$$

In the field of view, one snapshot is considered as a high-dimensional vector and then arranged into a one-column matrix e. So the residual of the convergence within the field of view is obtained by the difference of the Euclidean norm:

$$
\begin{gathered}
r_{\text {mean }}=\left\|\frac{1}{N} \sum_{i=1}^{N} \mathbf{e}_{i}\right\|_{2}-\left\|\frac{1}{N-100} \sum_{i=1}^{N-100} \mathbf{e}_{\mathbf{i}}\right\|_{2} \\
r_{\text {r.m.s. }}=\left\|\sqrt{\frac{1}{N-1} \sum_{i=1}^{N}\left(\mathbf{e}_{i}-\overline{\mathbf{e}}\right)^{2}}\right\|_{2}-\left\|\sqrt{\frac{1}{(N-100)-1} \sum_{i=1}^{N-100}\left(\mathbf{e}_{\mathbf{i}}-\overline{\mathbf{e}}\right)^{2}}\right\|_{2}
\end{gathered}
$$

The convergence history of the mean and r.m.s. velocities are shown in Figure 4- 4. The time-averaged streamwise velocities are converged to constant values very well after exceeding over 1,000 snapshots. The time-averaged vertical velocities are also converged well with limited variations of less than $0.1 \mathrm{~m} / \mathrm{s}$. Meanwhile, the fluctuations in the streamwise and vertical directions are converged to constant values. The points within the shear layer have more turbulent fluctuations than those outside. On the other hand, the convergence of the field of view is shown in Figure 4- 5. It is apparent that, as the number of snapshots increases to 4,000, the time-average and turbulent fluctuations within the shear layer are well converged to limited variations in the range of less than $0.2 \mathrm{~m} / \mathrm{s}$. Thus, it can be concluded that the 4,000 uncorrelated snapshots in each case satisfy the convergence condition for statistical calculations. 



Figure 4- 2 Velocity contours of the clean case of the BFS flow. (a) mean velocity $\overline{\mathrm{u}} / \mathrm{U}_{0}$; (b) r.m.s. velocity $\mathrm{u}_{\text {r.m.s. }} / \mathrm{U}_{0}$

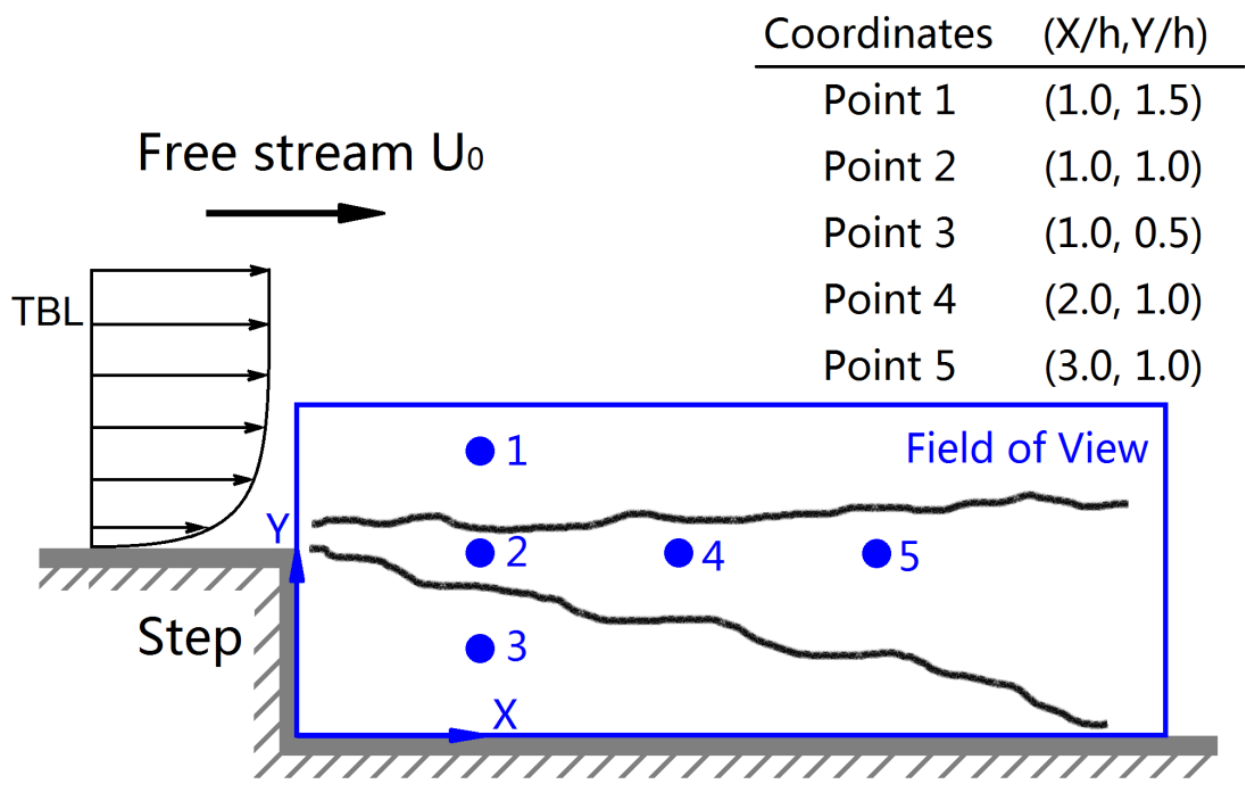

Figure 4- 3 Sketch of the five discrete points and field of view 

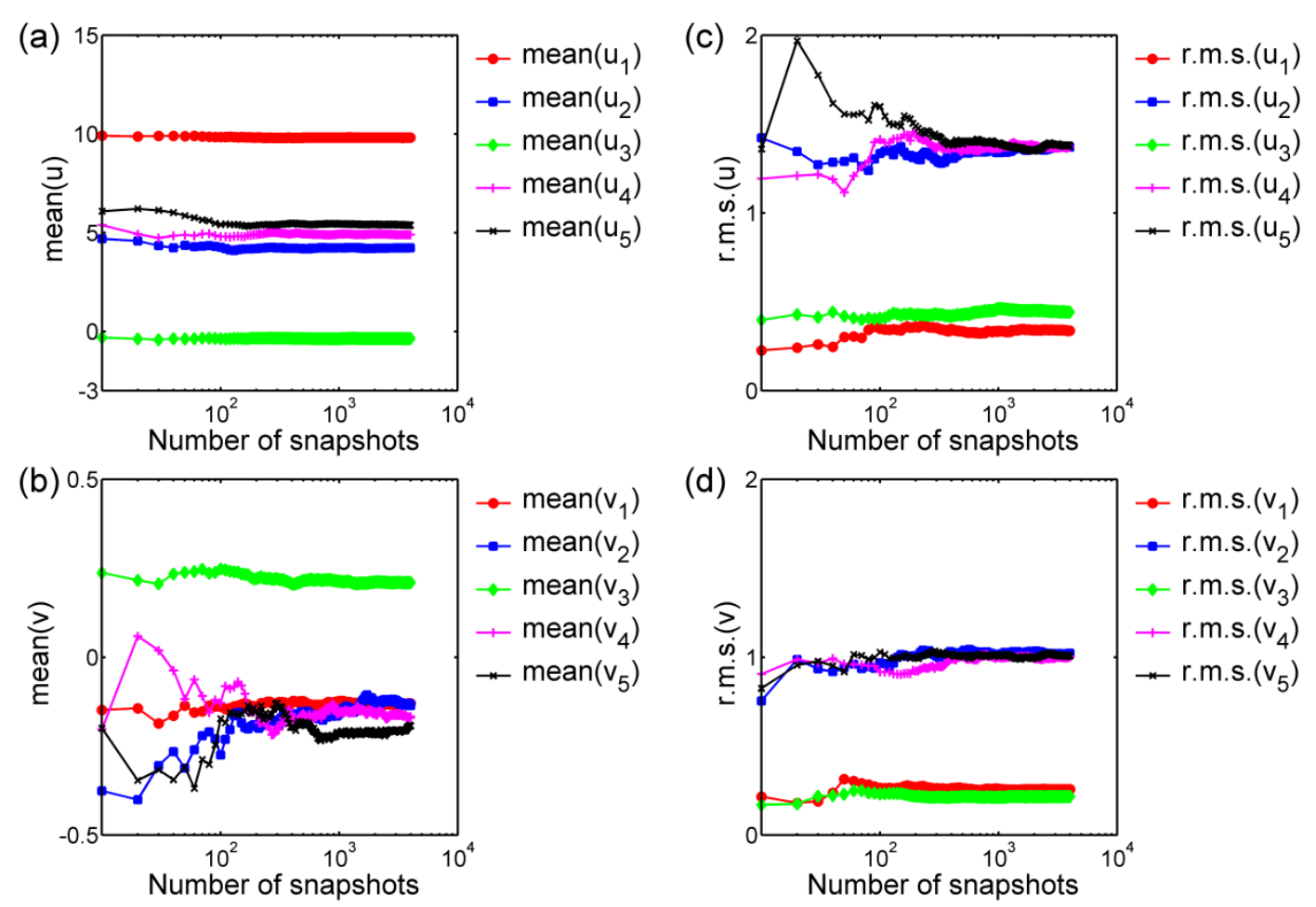

Figure 4- 4 Convergence of mean and r.m.s. velocities at five discrete points

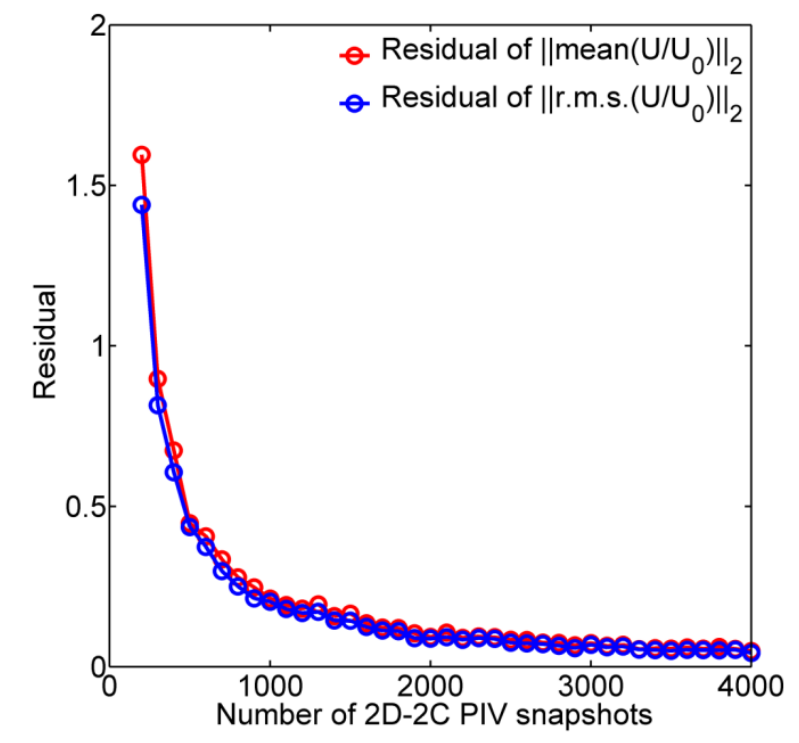

Figure 4- 5 Convergence history of the mean and r.m.s. velocities in the field of view

\subsection{Parameter study and flow control result}

In the parameter study, the main parameters include the non-dimensional actuation frequency $\mathrm{St}_{\mathrm{h}}=\mathrm{f}_{\mathrm{a}} \cdot \mathrm{h} / \mathrm{U}_{0}$ and the sound pressure level SPL which measures velocity amplitude of the 
actuations. The parameter sets as well as corresponding reductions of reattachment length are listed in Table 4- 1 and shown in Figure 4- 9. These two parameters have different influences on the flow control effect. Phase-averaged vortex structures at different frequencies and the same SPL are compared in Figure 4- 6 to show the frequency response of the turbulent shear layer. If the actuation frequency is at $\mathrm{St}_{\mathrm{h}}=0.3\left(\mathrm{f}_{\mathrm{a}}=100 \mathrm{~Hz}\right)$, which is close to the most-amplified frequency of the turbulent shear layer (Ma et al. 2014b), apparent spanwise vortices are generated due to the periodic actuation. The reattachment length is $L / h=4.0$. The corresponding Strouhal number based on the momentum thickness is $\mathrm{St}_{\theta}=0.02$, which is a little higher than the "shear layer mode" at $\mathrm{St}_{\theta} \approx 0.012$ for laminar separations (Hasan 1992). Table 4- 2 shows the comparison of non-dimensional frequencies and reattachment lengths in the present experiment and the literature. If the frequency increases to $\mathrm{St}_{\mathrm{h}}=0.9$, the periodic vortices become much smaller and decay quickly downstream. The reattachment length increases to $\mathrm{L} / \mathrm{h}=6.5$. For even higher frequencies $\mathrm{St}_{\mathrm{h}} \geq 1.5$, the periodic vortices are hardly detected and the reattachment length increases to $\mathrm{L} / \mathrm{h}=7.3$, which is even larger than for the clean case of $\mathrm{L} / \mathrm{h}=7.1$. This agrees well with the experimental results reported by Chun et al. (1996) that the effective perturbation was at $\mathrm{St}_{\mathrm{h}}=0.29$ and higher frequencies above $\mathrm{St}_{\mathrm{h}} \geq 1.0$ may even increase the reattachment length. On the other hand, phase-averaged vortex structures at the same frequency and different SPLs are compared in Figure 4- 7 to show the amplitude response of the turbulent shear layer. The respective reattachment lengths are $\mathrm{L} / \mathrm{h}=4.3,4.0,4.0$. As the SPL increases, the vortex structures have increasing scales and similar formations, but the reattachment length remains almost the same. Therefore, based on the reduction of the reattachment length among the tested parameter sets, the case at $\mathrm{St}_{\mathrm{h}}=0.3$ and $\mathrm{SPL}=110 \mathrm{~dB}$ is selected as a "controlled case" for further analysis. 

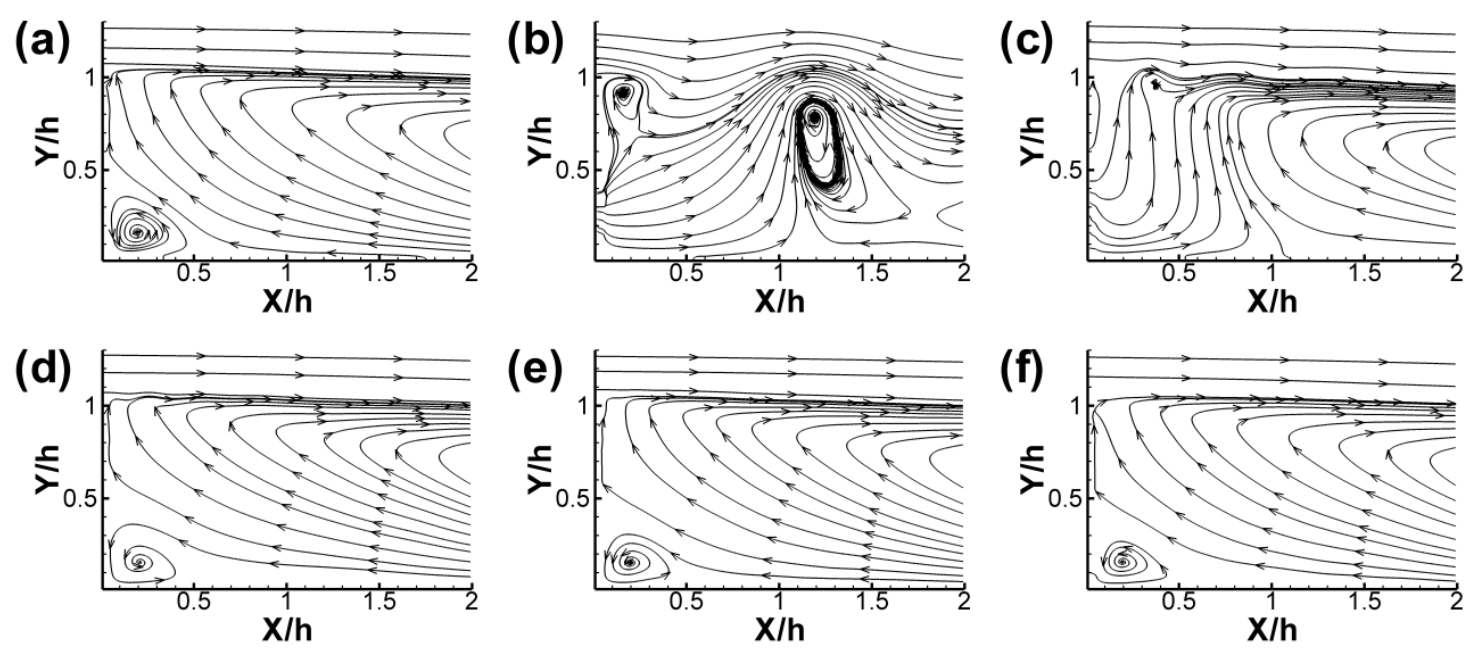

Figure 4- 6 Phase-averaged streamlines at the same $S P L=110 \mathrm{~dB}$ and different frequencies. (a)-(f) $\mathrm{St}_{\mathrm{h}}=0$, $0.3,0.9,1.5,2.1$ and 2.7
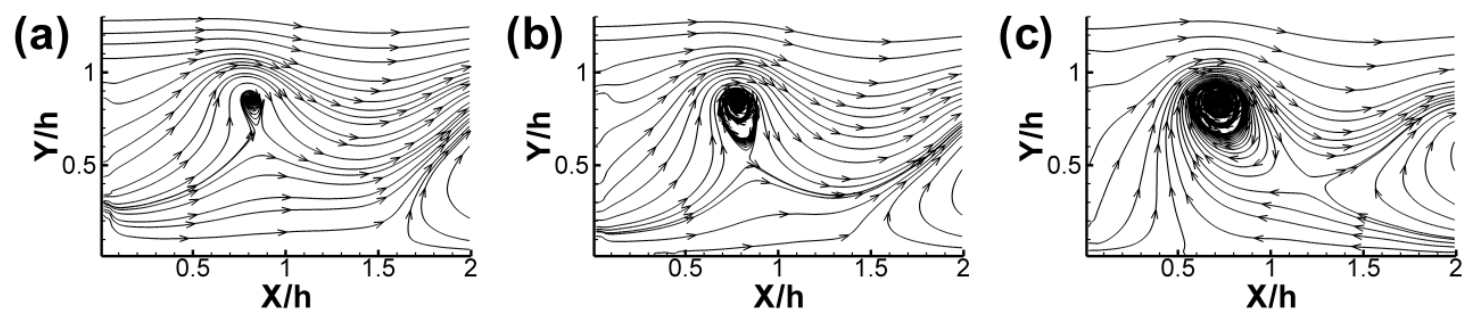

Figure 4- 7 Phase-averaged streamlines at the same frequency $\mathrm{St}_{\mathrm{h}}=0.3$ and different SPLs. (a)-(c) $\mathrm{SPL}=$ 105,110 and $115 \mathrm{~dB}$

Table 4- 1 Reduction of reattachment length of different parameter sets

\begin{tabular}{llllll}
\hline \hline Case & AT & $\mathrm{f}_{\mathrm{a}}[\mathrm{Hz}]$ & $\mathrm{SPL}[\mathrm{dB}]$ & $\mathrm{L} / \mathrm{h}$ & Label \\
\hline clean BFS & off & $/$ & $/$ & 7.1 & clean case \\
controlled BFS 1 & on & 100 & 110 & $\mathbf{4 . 0 2}$ & controlled case \\
controlled BFS 2 & on & 100 & 105 & 4.30 & $/$ \\
controlled BFS 3 & on & 100 & 115 & 4.02 & $/$ \\
controlled BFS 4 & on & 300 & 110 & 6.53 & $/$ \\
controlled BFS 5 & on & 500 & 110 & 7.53 & $/$ \\
controlled BFS 6 & on & 700 & 110 & 7.17 & $/$ \\
controlled BFS 7 & on & 900 & 110 & 7.12 & $/$ \\
\hline \hline
\end{tabular}


Table 4- 2 Reduction of reattachment length in the present experiment and the literature

\begin{tabular}{lllllll}
\hline \hline Author(s) & Control method & $\mathrm{St}_{\mathrm{h}}$ & $\mathrm{St}_{\theta}$ & $\mathrm{L}_{0} / \mathrm{h}$ & $\mathrm{L} / \mathrm{h}$ & $\mathrm{R}_{\mathrm{L}}$ \\
\hline Roos et al. (1986) & mechanical & 0.29 & $/$ & 5.5 & 4.5 & $18.2 \%$ \\
Bhattacharjee et al. & acoustic & 0.28 & 0.005 & 6.0 & 5.0 & $16.7 \%$ \\
$\begin{array}{l}\text { (1986) } \\
\text { Hasan (1992) }\end{array}$ & acoustic & $0.14-0.85$ & $0.003-0.02$ & $/$ & $/$ & $/$ \\
Chun et al. (1996) & acoustic & 0.29 & 0.01 & 7.2 & 4.68 & $35.0 \%$ \\
Present & acoustic & 0.3 & 0.02 & 7.1 & 4.0 & $\mathbf{4 3 . 7 \%}$ \\
\hline \hline
\end{tabular}

Time-averaged velocity vector fields of the clean and controlled cases are compared in Figure 4- 8. In the clean case, the main and the secondary recirculation regions are identified behind the step and the reattachment length is $\mathrm{L}_{0} / \mathrm{h}=7.1$. In the controlled case, the non-dimensional frequency is $\mathrm{St}_{\mathrm{h}}=0.3$ and the ratio of the root-mean-square of the fluctuating velocity magnitude to free-stream velocity is $|\mathrm{U}|_{\mathrm{rms}} / \mathrm{U}_{0}=0.18$. The comparison shows the streamlines are drawn downward closer to the wall. The main recirculation region is clearly suppressed and its center position moves upstream and closer to the wall from the point $(\mathrm{X} / \mathrm{h}=3.74, \mathrm{Y} / \mathrm{h}=0.47)$ to $(\mathrm{X} / \mathrm{h}=2.57, \mathrm{Y} / \mathrm{h}=0.30)$. The reattachment length is reduced to $\mathrm{L} / \mathrm{h}=4.0$, resulting in $\mathrm{a}$ reduction rate of $\mathrm{R}_{\mathrm{L}}=43.7 \%$. It is noted that the secondary recirculation region disappears and the time-averaged streamlines start from the step wall, as shown in Figure 4- 8(b). One reason for the three-dimensional spanwise mean flow is that the spanwise limited acoustic actuations could entrain neighboring fluid of two sides into the low-pressure low-speed recirculation region. As a result the recirculation region is smaller than that of the clean case. The other reason is the asymmetric design of the acoustic tube. It had one loudspeaker as sound source mounted on one side and a sealing by a hard plate on the other side. In that way the acoustic actuation along the slot is slightly asymmetric: it is found that the SPL along the slot shows quit small differences (a few $\mathrm{dB}$ ), compared to the $\mathrm{SPL}=110 \mathrm{~dB}$ at the center of the slot. 

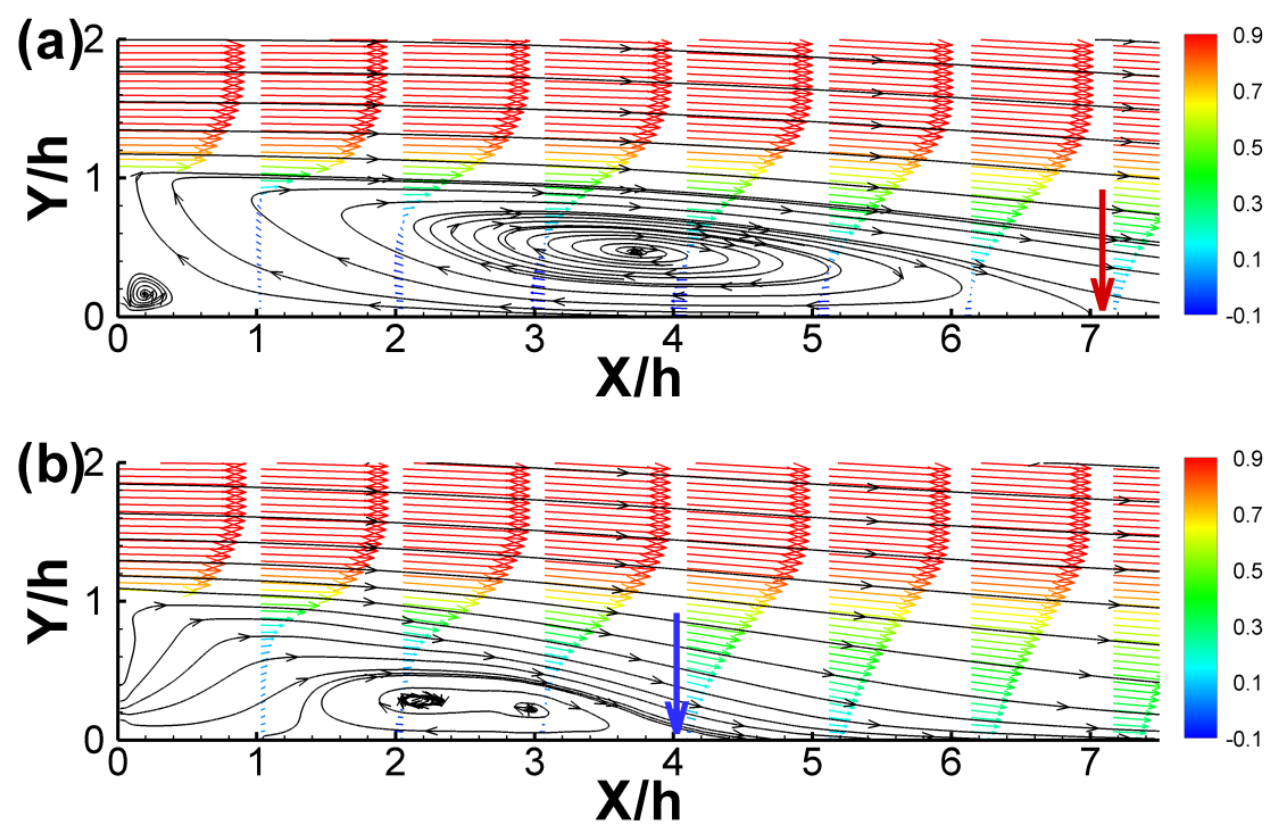

Figure 4- 8 Time-averaged velocity vector fields. (a) clean case; (b) controlled case. The arrows indicate the time-averaged reattachment points. The color indicates streamwise velocity component $\overline{\mathrm{u}} / \mathrm{U}_{0}$.
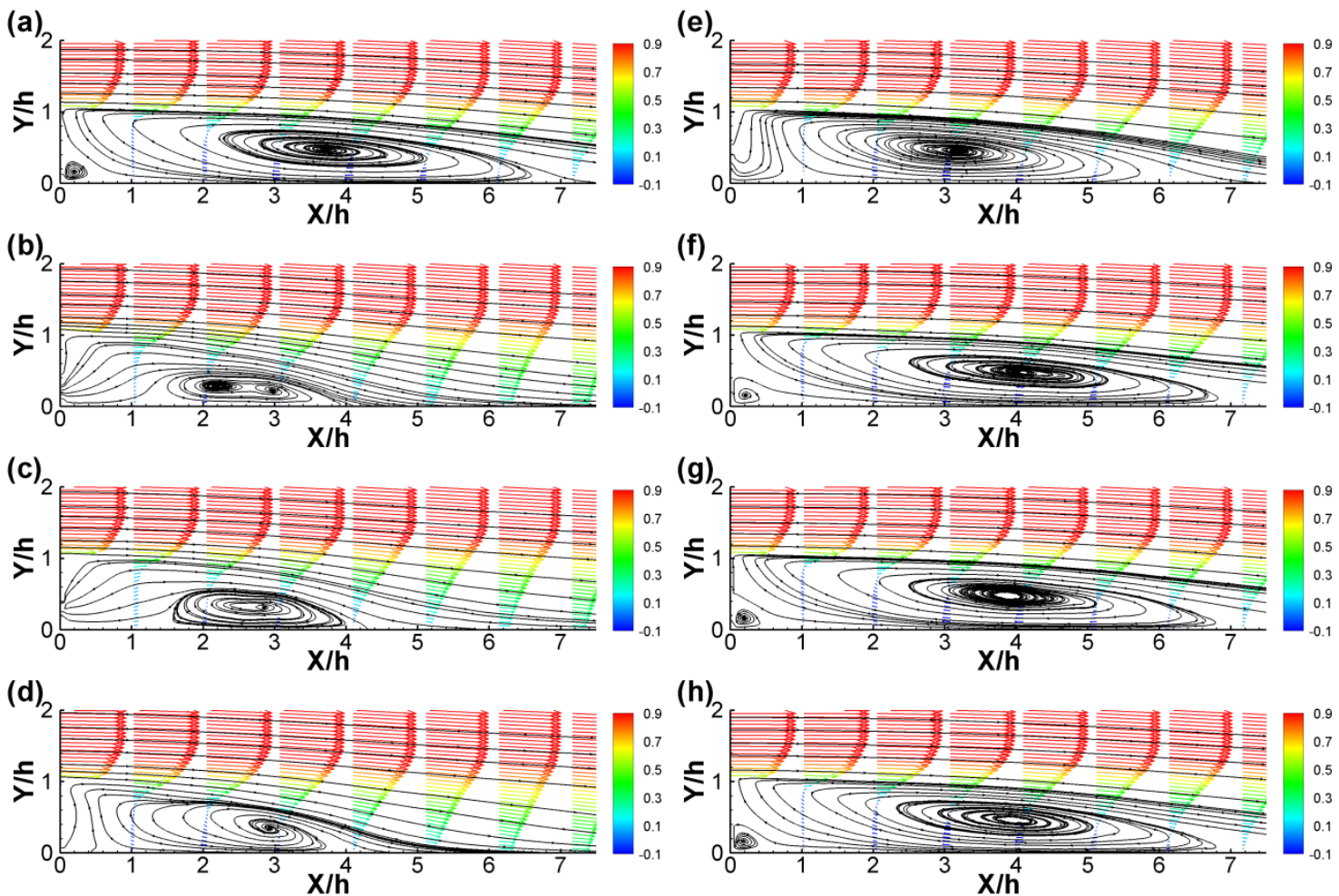

Figure 4- 9 Time-averaged velocity vector fields of the clean and the controlled cases (a) clean case; (b) $100 \mathrm{~Hz}, 110 \mathrm{~dB}$; (c) $100 \mathrm{~Hz}, 105 \mathrm{~dB}$; (d) $100 \mathrm{~Hz}, 115 \mathrm{~dB}$; (e) $300 \mathrm{~Hz}, 110 \mathrm{~dB}$; (f) $500 \mathrm{~Hz}, 110 \mathrm{~dB}$; (g) 700 $\mathrm{Hz}, 110 \mathrm{~dB}$; (h) $900 \mathrm{~Hz}, 110 \mathrm{~dB}$ 


\subsection{Phase-averaged flow fields}

The phase-locked measurements provide insight into the development of the coherent structures related to the periodic actuation in the turbulent shear layer. The phase-averaged velocity vector fields of the controlled case are shown in Figure 4- 10. Spanwise vortices roll up, approximately within $0<\mathrm{X} / \mathrm{h}<1.5$, at the beginning of the shear layer. The distance between the first two vortices is approximately $\Delta \mathrm{X} / \mathrm{h} \approx 1.0$. Further downstream approximately within $1.5<\mathrm{X} / \mathrm{h}<4$, the vortices move downstream and toward the wall with a growing size and vortex pairing occurs near the wall. The distance between two vortices increases to $\Delta \mathrm{X} / \mathrm{h} \approx 1.5$. The phase-averaged streamlines of the outer flow are drawn closer to the wall.
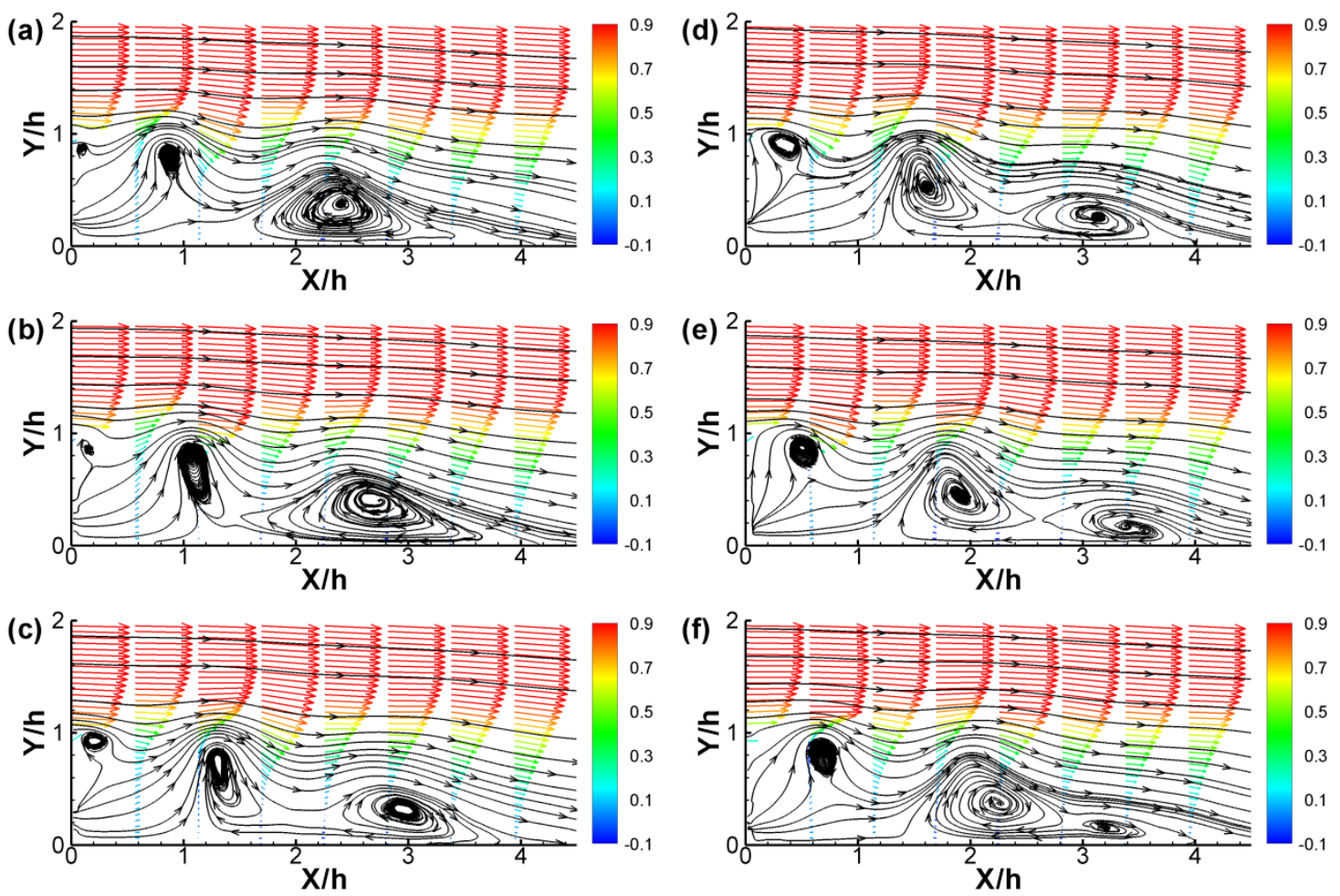

Figure 4- 10 Phase-averaged flow vector fields of the controlled case. (a)-(f) $0^{\circ}, 60^{\circ}, 120^{\circ}, 180^{\circ}, 240^{\circ}$ and $300^{\circ}$. The color indicates streamwise velocity component $\langle\mathrm{u}\rangle / \mathrm{U}_{0}$.

\subsection{Analysis of Reynolds shear stress}

Reynolds shear stress is an important quantity for momentum transfer in turbulent flows. It represents the quantity of the fluid mass fluctuating upward and downward between the shear 
layers (Schlichting 1979). Based on the triple decomposition, the Reynolds shear stress can be decomposed as:

$$
\tau_{\mathrm{xy}}^{\prime}=-\rho \cdot \overline{\left(\widetilde{\mathrm{u}}+\mathrm{u}^{\prime}\right) \cdot\left(\tilde{\mathrm{v}}+\mathrm{v}^{\prime}\right)}
$$

Additionally, the periodic components are uncorrelated with the random fluctuations:

$$
\overline{\tilde{\mathrm{u}} \cdot \mathrm{v}^{\prime}}=0, \overline{\mathrm{u}^{\prime} \cdot \tilde{\mathrm{v}}}=0
$$

So the Reynolds shear stress is decomposed as:

$$
\tau_{x y}^{\prime}=-\rho \cdot\left(\overline{\tilde{u} \cdot \tilde{v}}+\overline{u^{\prime} \cdot v^{\prime}}\right)
$$

On the right-hand side the first part $\overline{\mathrm{u}} \cdot \tilde{\mathrm{v}}$ is the contribution of the coherent structures, and the second part $\overline{\mathrm{u}^{\prime} \cdot \mathrm{v}^{\prime}}$ is the contribution of the incoherent turbulence.
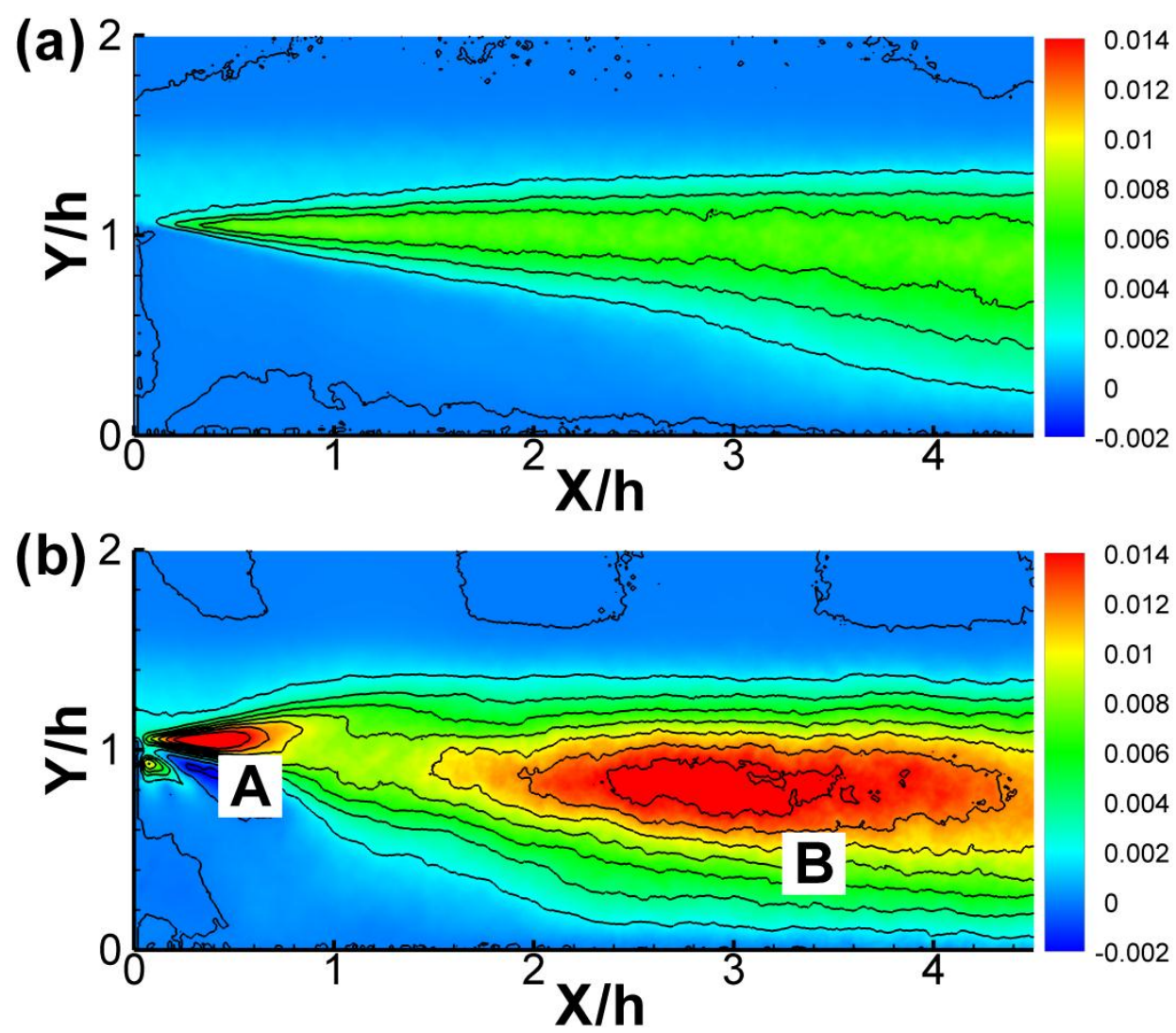

Figure 4- 11 Total Reynolds shear stress $-\left(\overline{\tilde{u} \cdot \tilde{v}}+\overline{u^{\prime} \cdot v^{\prime}}\right) / U_{0}^{2}$. (a) clean case; (b) controlled case

The comparison shows that the total Reynolds shear stress is increased by the periodic 
actuations and there are two distinct high-value regions " $\mathrm{A}$ " and " $\mathrm{B}$ " in the controlled case

(Figure 4- 11). The contributions of the coherent and incoherent motions are compared in Figure 4- 12 (Ma et al. 2015). The spanwise vortices roll up near the acoustic tube at approximately $\mathrm{X} / \mathrm{h} \approx 0.5$ and contribute to the major part of the high Reynolds shear stress in the region "A". On the other hand, incoherent turbulence contributes to Reynolds shear stress in the entire shear layer and the vortex pairing and breakdown result in higher Reynolds shear stress in the region "B". Therefore, the coherent structure play an important role in the transports of mass and momentum in "its own territory" (Hussain 1986) and delivers comparable contributions as the incoherent turbulence.
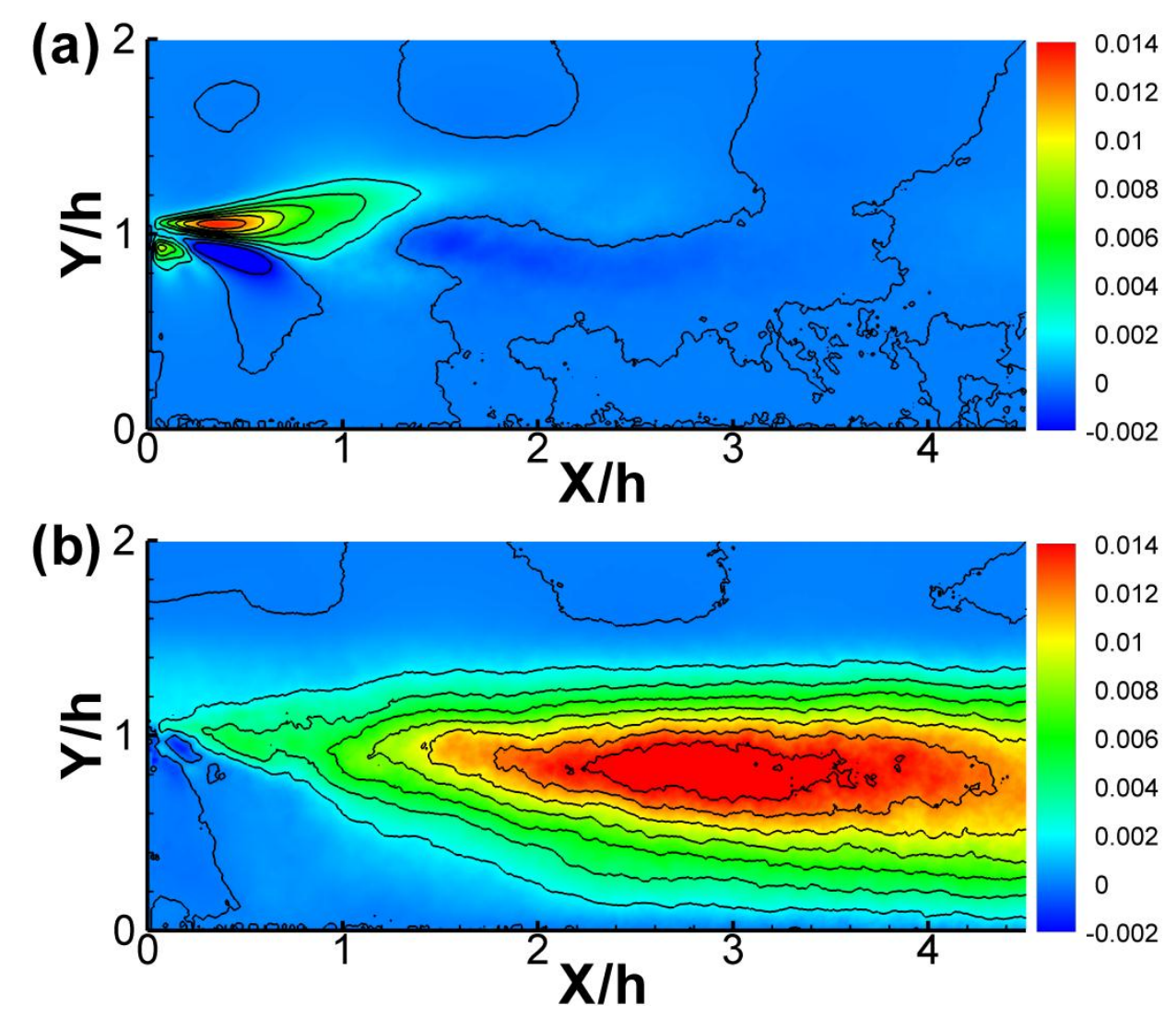

Figure 4- 12 Decomposition of Reynolds shear stress of the controlled case. (a) coherent quasi-periodic part $-\overline{\tilde{\mathrm{u}} \cdot \tilde{\mathrm{v}}} / \mathrm{U}_{0}^{2} ;(\mathbf{b})$ incoherent part $-\overline{\mathrm{u}^{\prime} \cdot \mathrm{v}^{\prime}} / \mathrm{U}_{0}^{2}$

The incoherent Reynolds shear stresses are obtained from the phase-locked flow fields, as shown in Figure 4- 13. The interface between the shear layer and the outer flow is evolving 
with the movements of the spanwise vortices. It is clear that the vortex roll-up and breakdown play predominant roles in the shear layer and both processes are related to the increase of Reynolds shear stress. The high momentum fluid mass is entrained due to the Biot-Savart induction and then is engulfed into the turbulent shear layer (Hussain 1986). This entrainment produces more turbulent motions in the shear layer than turbulent mixing processes of the clean case, which results in a larger spread of the shear layer and a significant reduction of the reattachment length.
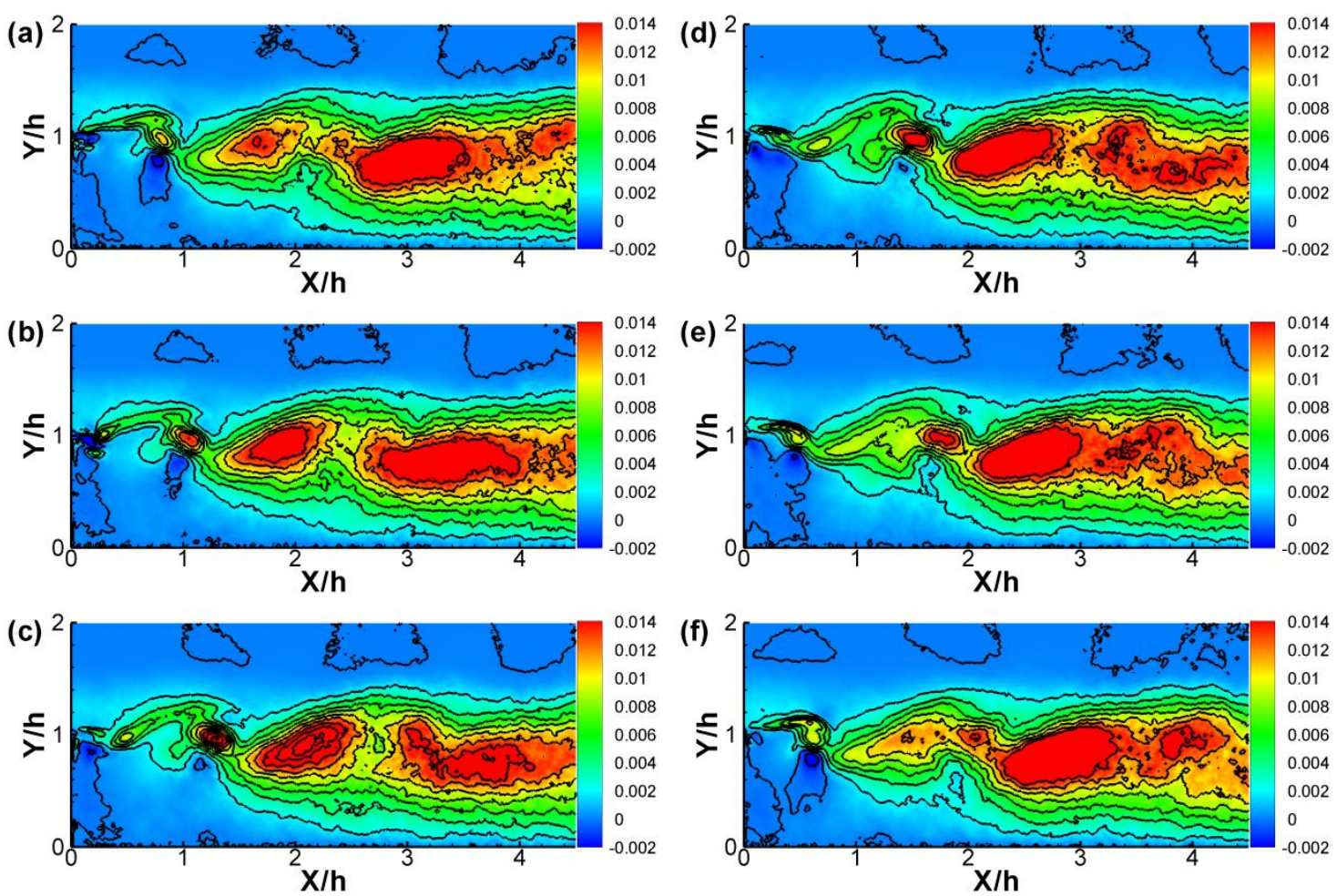

Figure 4- 13 Phase-averaged incoherent Reynolds shear stress $-\left\langle u^{\prime} \cdot v^{\prime}\right\rangle / U_{0}^{2}$ of the controlled case. (a)-(f) $0^{\circ}, 60^{\circ}, 120^{\circ}, 180^{\circ}, 240^{\circ}$ and $300^{\circ}$

\subsection{Spatial cross-correlation function}

Figure 4- 14 presents the spatial two-point cross-correlation functions of the vertical velocity component. The normalized coordinates of the reference points are $X_{0} / h=1.0,2.0,3.0$ and $Y_{0} / h$ $=$ 1.0. In the clean case, as shown in Figure 4- 14(a)-(c), the elliptical regions of positive correlation is at the reference points of $\mathrm{X}_{0} / \mathrm{h}=1.0$ and two adjacent regions of negative 
correlation are locate upstream and downstream, though the negative correlation is weak. The rest part of the flow field shows little correlation features due to the turbulent mixing motions. As the reference point moves downstream to $\mathrm{X}_{0} / \mathrm{h}=2.0$ and 3.0, the distance of the correlated regions increases. This is an indication that the spatial scale of the vortices within the shear layer increases as the vortices move downstream.

In the controlled case, a clear correlation pattern consisting of positive and negative correlation regions is shown in Figure 4- 14(d)-(f). The large-scale coherent structure has an approximate size of two spatial periods in the streamwise direction within $0<\mathrm{X} / \mathrm{h}<2$, which agrees well with the phase-averaged spanwise vortex structures in Figure 4- 10. In Figure 4- 14(f), the vortices are less-correlated as the reference point moves further downstream, which is mainly due to the attenuation of vortex strength, the incoherent turbulent mixing and the out-of-plane motions. Thus, the correlation results characterize the spatial scale and evolution of the coherent structures in the controlled case.


Figure 4- 14 Contours of coefficient of spatial cross-correlation. (a)-(c) $R_{v^{\prime} v^{\prime}}$ of the clean case; (d)-(f) $\mathrm{R}_{\mathrm{v}^{\prime} \mathrm{v}^{\prime}}$ of the controlled case. The reference points are at $\mathrm{Y}_{0} / \mathrm{h}=1.0$ and $\mathrm{X}_{0} / \mathrm{h}=1.0,2.0,3.0$, respectively. 


\subsection{Analysis of coherent structures}

POD is an effective method for identifying coherent structures in the flow by linear decomposition and reconstruction (Lumley 1967). In order to reveal the spatial structures of the vortices, the snapshot POD method (Sirovich 1987) is applied in a rectangular region with a size of $1.7 \mathrm{~h} \times 1 \mathrm{~h}$ (Figure 4- 15), which focuses on the induction of the coherent structures in the initial part of the shear layer. Detailed mathematical algorithms of the snapshot POD method is presented by Meyer et al. (2007). This method decomposes the original velocity data sequence into the mean flow and the linear combination of spatial orthogonal modes. All the modes are ranked decreasingly based on the eigenvalues, which represent the turbulent kinetic energy (Meyer et al. 2007). This energy-based hierarchy ensures that predominant and large-scale flow structures can be represented by the first few modes. In the present analysis, the number of instantaneous velocity vector fields that are used is $\mathrm{N}=4.000$ and each snapshot contains 15,974 spatial points.

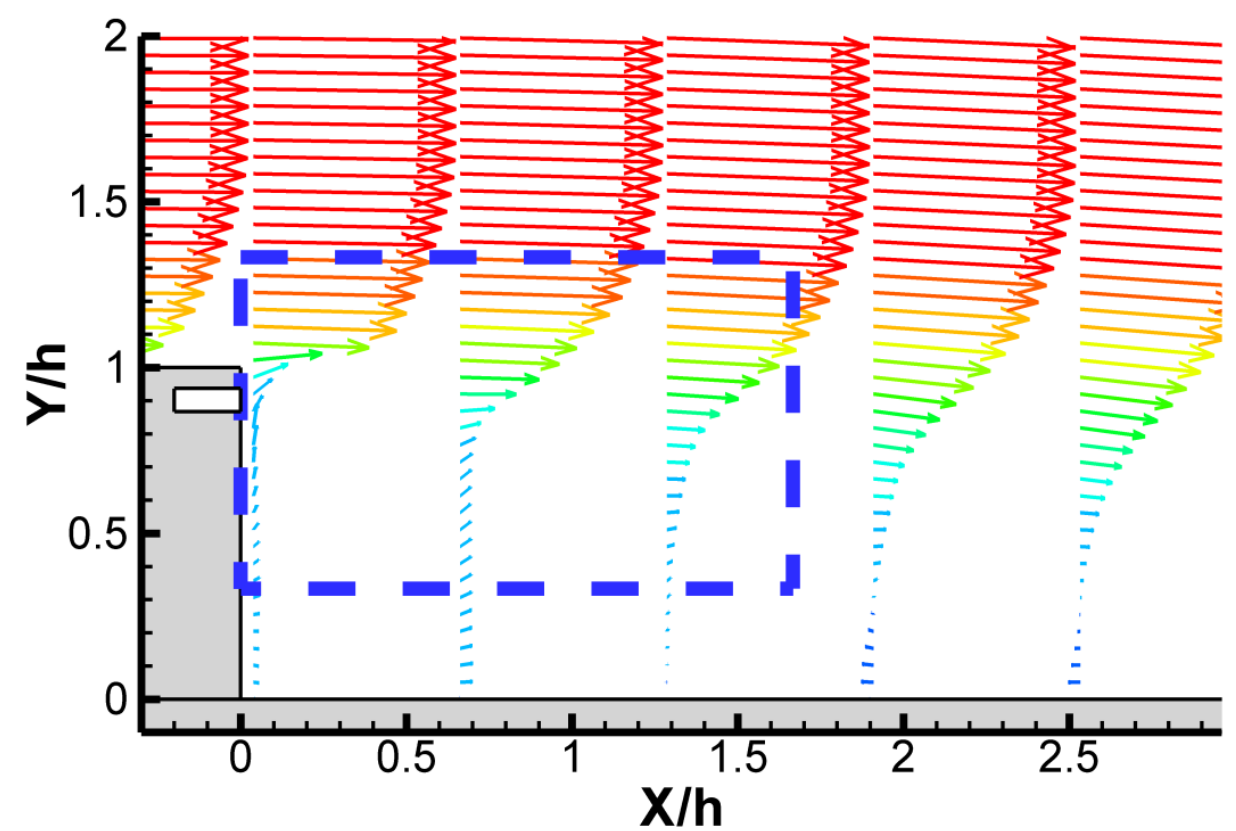

Figure 4- 15 Sketch of the rectangular region of $1.7 \mathrm{~h} \times 1 \mathrm{~h}$ in which POD is applied

The POD eigenvalue distributions of the clean and controlled cases are plotted in Figure 4- 16. For both cases the first two modes contain the most turbulent kinetic energy and the other 
modes decay exponentially. The comparison shows the energetic fluctuating motions of the controlled case are larger than those of the clean case. Additionally, the first two modes of the controlled case contain nearly equivalent energy. By scattering the coefficients of two modes, the coherent phase interrelations can be revealed. Three typical pairs of modes are depicted by the ellipses in Figure 4- 17, which are $\mathrm{POD}_{1}$ and $\mathrm{POD}_{2}$ of the clean case, $\mathrm{POD}_{1}$ and $\mathrm{POD}_{2}$, $\mathrm{POD}_{5}$ and $\mathrm{POD}_{6}$ of the controlled case. Two geometrical variables of a scatter point $\left(a_{\mathrm{i}}, \mathrm{a}_{\mathrm{j}}\right)$, phase angle $\varphi$ (Perrin et al. 2007) and radius r, are defined as:

$$
\begin{gathered}
\varphi_{i, j}=\arctan \left(\frac{\sqrt{\lambda_{i}}}{\sqrt{\lambda_{j}}} \cdot \frac{a_{j}}{a_{i}}\right) \\
r_{i, j}=\sqrt{a_{i}^{2}+a_{j}^{2}}
\end{gathered}
$$

In the clean case, the coefficients $a_{1}$ and $a_{2}$ gather to the center as a disk, which indicates that the two modes are independent and incoherent of each other with respect to a phase relation. In the controlled case, by contrast, the coefficients $\mathrm{a}_{1}$ and $\mathrm{a}_{2}$ are organized as a circle, which shows that the two modes are coherent and contain a fixed phase difference. A similar scatter distribution is also found for the coefficients $\mathrm{a}_{5}$ and $\mathrm{a}_{6}$ of the controlled case. The other modes of the controlled case do not contain this coherent feature.



Figure 4- 16 POD eigenvalue distributions of the first 100 modes 
In order to describe the radial distributions quantitatively, relative standard deviation (RSD) is used to measure how the coefficient points are scattered near the circle of the mean radius:

$$
\operatorname{RSD}=\frac{\sigma_{\mathrm{r}}}{\overline{\mathrm{r}}} \times 100 \%
$$

$\sigma_{\mathrm{r}}$ is the standard deviation of the radius $r$ and $\bar{r}$ is the mean value. Thus, the RSDs of the three cases are $51.9 \%, 14.7 \%$ and $37.1 \%$, respectively. The low value of $14.7 \%$ for $\mathrm{POD}_{1}$ and $\mathrm{POD}_{2}$ of the controlled case indicates that most of the points are close to the circle with the mean radius. The high value of $51.9 \%$ for $\mathrm{POD}_{1}$ and $\mathrm{POD}_{2}$ of the clean case indicates that all points vary far from the circle.
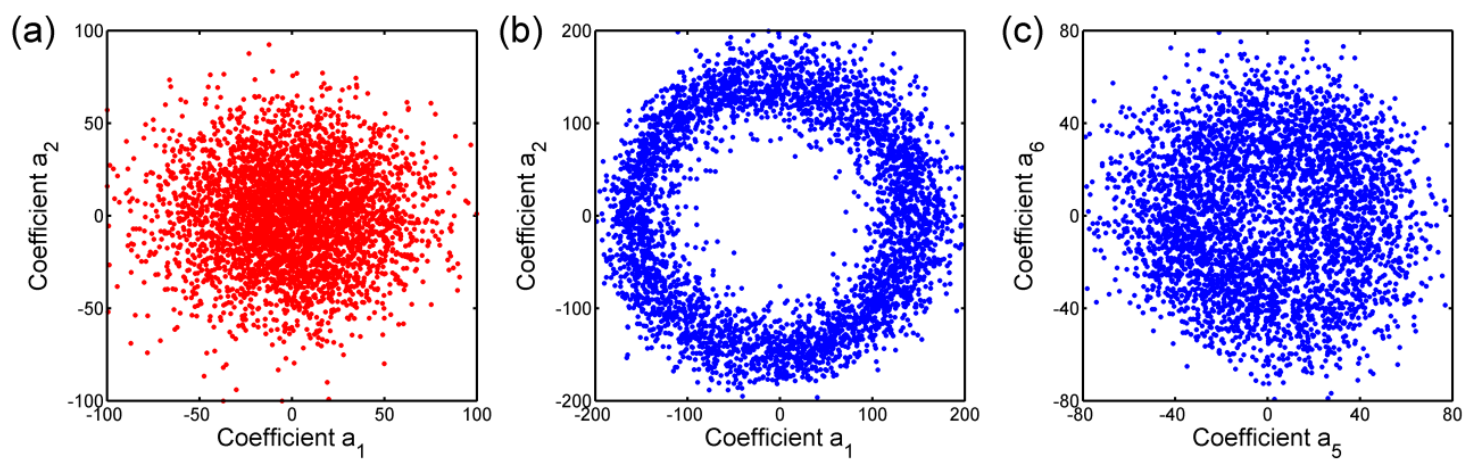

Figure 4- 17 Scatter distributions of POD coefficients. (a) $\mathrm{POD}_{1}$ and $\mathrm{POD}_{2}$ of the clean case; (b) $\mathrm{POD}_{1}$ and $\mathrm{POD}_{2}$ of the controlled case; (c) $\mathrm{POD}_{5}$ and $\mathrm{POD}_{6}$ of the controlled case

POD reconstruction can be obtained by linearly combining modes weighted by corresponding coefficients. As shown in Figure 4- 18 counter-rotating vortices at different phases are shown in the reconstruction by $\mathrm{POD}_{1}$ and $\mathrm{POD}_{2}$ of the controlled case. This regular pattern is a sign of the coherent structures represented by $\mathrm{POD}_{1}$ and $\mathrm{POD}_{2}$ which are mutually orthogonal and can be written as a composite form "POD $+\mathrm{i} \cdot \mathrm{POD}_{2}$ " (Schmid et al. 2012). In other words, the reconstructions are equivalent to the interference of two oscillating modes. An estimated wavelength of the vortices is $\Delta \mathrm{X} / \mathrm{h} \approx 1.0$, which agrees well with the spatial scale in the phase-averaged velocity vector fields in Figure 4- 10. Moreover, the reconstructions by $\mathrm{POD}_{5}$ and $\mathrm{POD}_{6}$ of the controlled case show the harmonic counter-rotating vortices with half the 
wavelength due to the overtone of the acoustic tube (Figure 4- 19). The phase relation between $\varphi_{1,2}$ and $\varphi_{5,6}$ in Figure 4- 20 are plotted as a regular pattern other than a uniform distribution, which indicates that the secondary vortices " $\mathrm{POD}_{5}+\mathrm{i} \cdot \mathrm{POD}_{6}$ " have doubled frequency of the primary vortices "POD $1+\mathrm{i} \cdot \mathrm{POD}_{2}$ ". These vortices show the distinct frequencies and coherent features, which is totally different from the wide frequency bandwidth and multi-scale structures as the natural behavior of the clean BFS flow (Bhattacharjee et al. 1986). Thus, it can be concluded that the primary and secondary series of vortices correspond to the fundamental frequency and the harmonic of the periodic actuations.

Therefore, some remarks of the acoustic tube are discussed. First, the acoustic tube generates periodic actuations based on the standing waves of the air inside the tube, which is different from direct acoustic excitations or synthetic jets in previous literature. Second, as a flow control device, the acoustic tube is able to reduce the time-averaged reattachment length by $43.7 \%$ in the BFS flow, which demonstrates its flow control effectiveness. Third, the periodic perturbations induce quasi-periodic spanwise vortices shedding downstream. The vortex rolling-up and breaking down result in an increase of the Reynolds shear stress. The entrainment by the coherent structures is more effective in the momentum transfer than the turbulent mixing due to the natural turbulent shear layer development. 

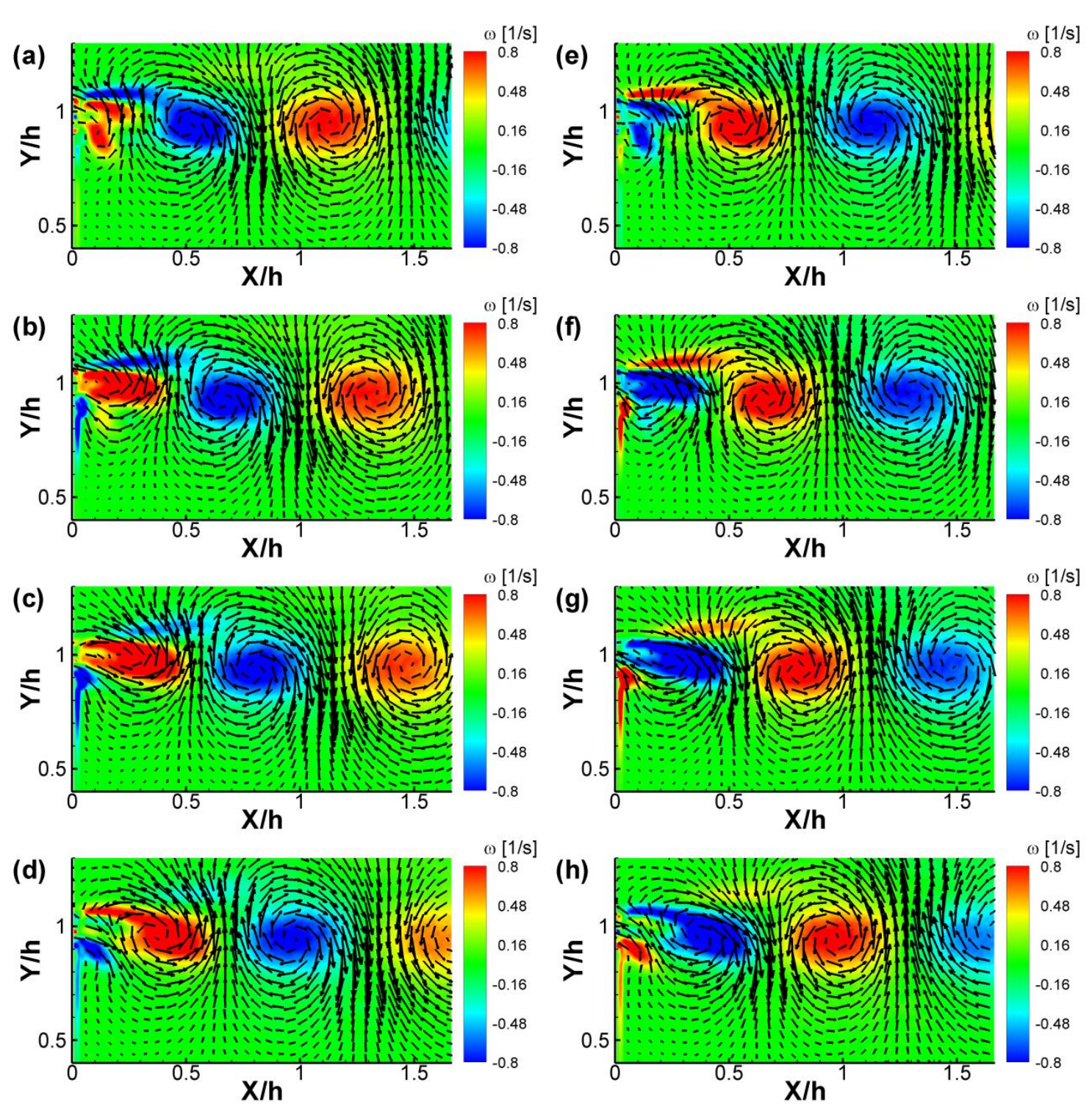

Figure 4- 18 Reconstructed by $\mathrm{POD}_{1}+\mathrm{i} \cdot \mathrm{POD}_{2}$ of the controlled case. (a)-(h) $0^{\circ}, 45^{\circ}, 90^{\circ}, 135^{\circ}, 180^{\circ}, 225^{\circ}$,

$270^{\circ}$ and $315^{\circ}$. The color indicates spanwise vorticity. Every 5th vector is shown for visual clarity. 



Figure 4- 19 Reconstructed by $\mathrm{POD}_{5}+\mathrm{i} \cdot \mathrm{POD}_{6}$ of the controlled case. (a)-(h) $0^{\circ}, 45^{\circ}, 90^{\circ}, 135^{\circ}, 180^{\circ}, 225^{\circ}$, $270^{\circ}$ and $315^{\circ}$. The color indicates spanwise vorticity. Every 5 th vector is shown for visual clarity. 


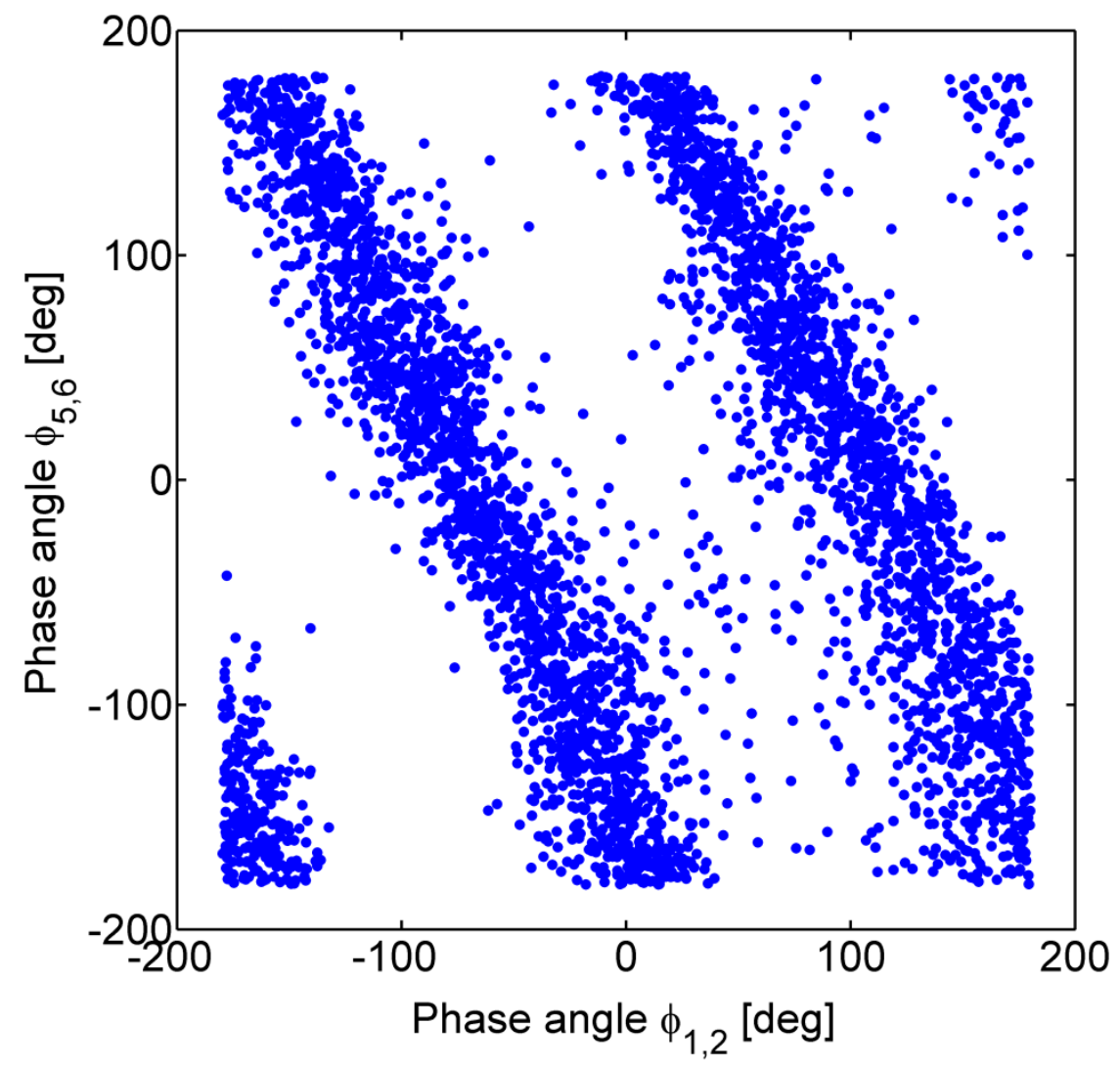

Figure 4- 20 Phase angle relation between primary and secondary vortices

"It is not very likely that science will ever achieve a complete understanding of the mechanism of turbulence because of its extremely complicated nature."

Hermann Schlichting (1907-1982) 


\section{Results of oscillating flap}

\subsection{Time-averaged velocity fields}

Time-averaged velocity vectors and contours of the clean and controlled cases are obtained by the non-phase-locked velocity data as shown in Figure 5- 1.
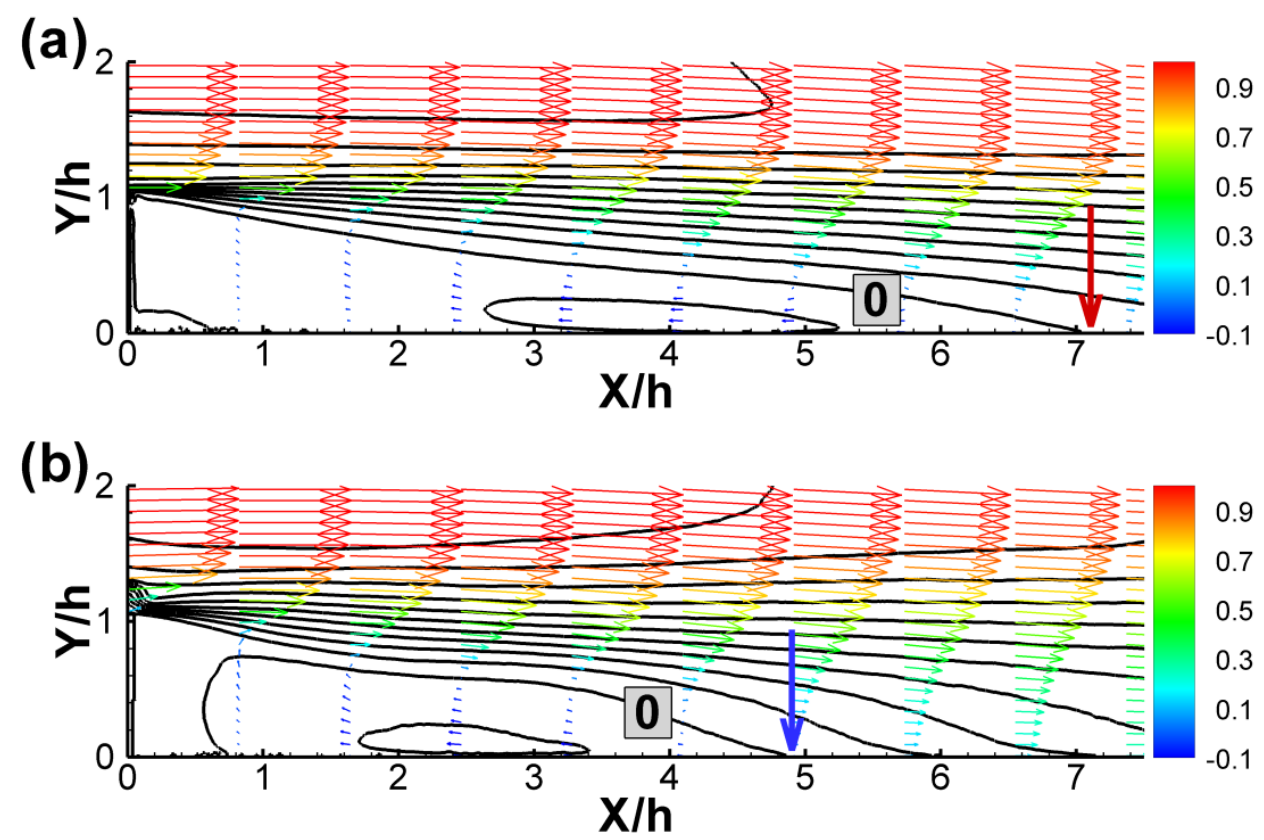

Figure 5- 1 Time-averaged velocity vector and contour fields. (a) clean case; (b) controlled case. The zero-velocity contour lines are labeled and the arrows indicate the time-averaged reattachment points. For clear visibility only $0.2 \%$ vectors are shown and the color indicates streamwise velocity component $\bar{u} / U_{0}$. 
In the clean case, the shear layer separates from the step and reattaches on the plate downstream at $\mathrm{X} / \mathrm{h}=7.1$, as marked by the red arrow. In the controlled case, the shear layer is drawn downward closer to the wall and the recirculation region is suppressed. As a result, the reattachment length is reduced to $\mathrm{X} / \mathrm{h}=4.9$ which corresponds to a reduction rate of $\mathrm{R}_{\mathrm{L}}=31.0 \%$. It is noted in Figure 5- 1(b) that the zero-velocity contour line is detached from the step edge and there is time-averaged streamwise flow near the step wall for the controlled case. It is clear that three-dimensional mean flow exists near the step. The reason for the three-dimensional mean flow is that the spanwise limited perturbations could entrain neighboring fluid into the low-pressure low-speed recirculation region, resulting in the smaller recirculation region downstream of the step.

\subsection{Analysis of Reynolds shear stress}
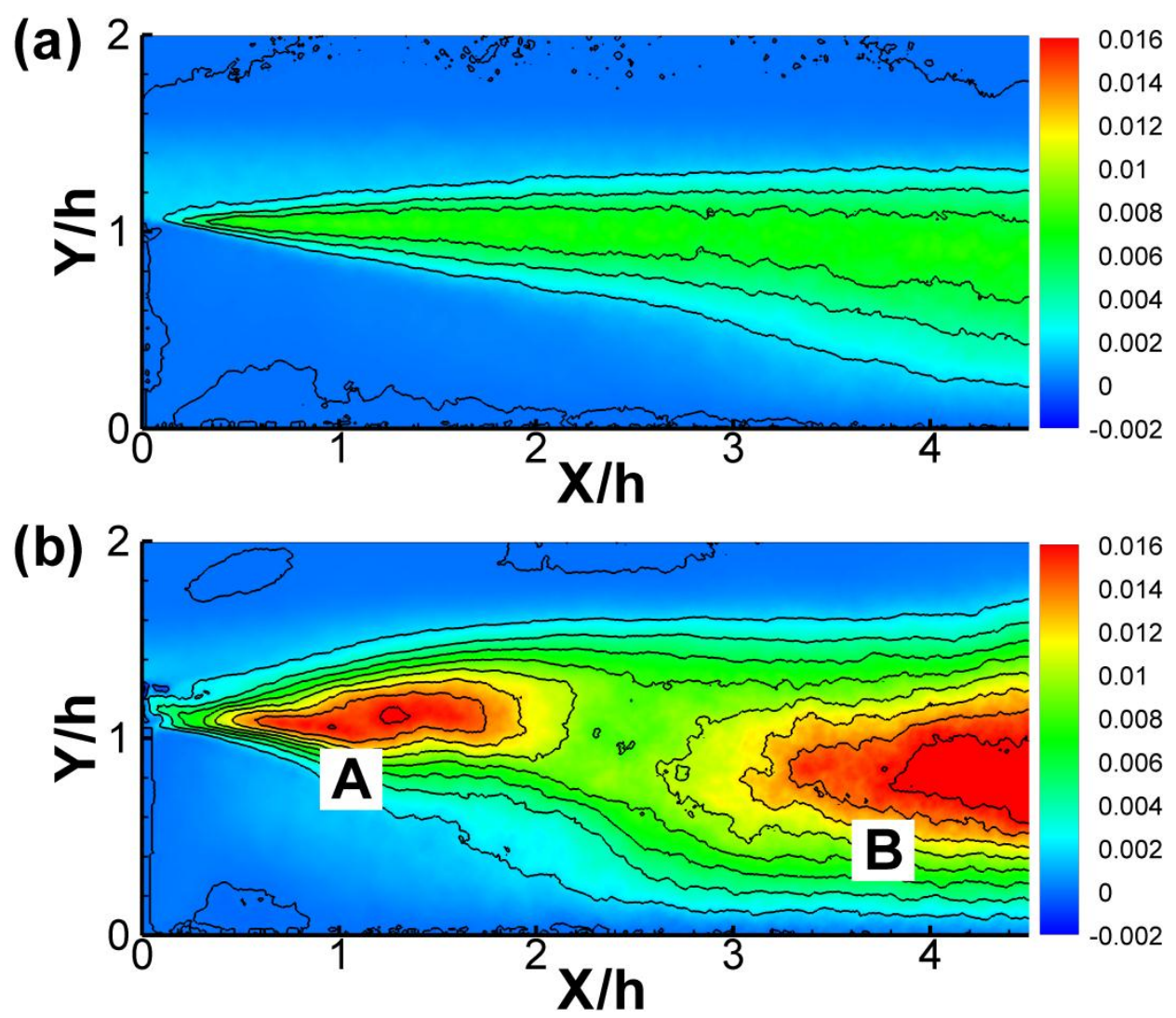

Figure 5- 2 Total Reynolds shear stress $-\left(\overline{\mathrm{u} \cdot \tilde{\mathrm{v}}}+\overline{\mathrm{u}^{\prime} \cdot \mathrm{v}^{\prime}}\right) / \mathrm{U}_{0}^{2}$. (a) clean case; (b) controlled case 
The comparison in Figure 5- 2 shows that the total Reynolds shear stress is increased by the periodic perturbations and there are two distinct high-value regions " $\mathrm{A}$ " and " $\mathrm{B}$ " in the controlled case. The contributions of the coherent structures and incoherent turbulence are compared in Figure 5- 3. The spanwise vortices roll up near the oscillating flap at approximately $\mathrm{X} / \mathrm{h} \approx 1.5$ and contribute to the major part of the high Reynolds shear stress in the region "A". On the other hand, incoherent turbulence contributes to Reynolds shear stress in the entire shear layer and the vortex breakdown leads to higher Reynolds shear stress in the region "B". The high momentum fluid is entrained into the shear layer due to the flapping motion, which produces more fluctuating motions than the turbulent mixing processes in the clean case.


Figure 5- 3 Decomposition of Reynolds shear stress of the controlled case. (a) coherent part $-\tilde{\tilde{u}} \cdot \tilde{\mathrm{v}} / \mathrm{U}_{0}^{2}$; (b) incoherent part $-\overline{\mathrm{u}^{\prime} \cdot \mathrm{v}^{\prime}} / \mathrm{U}_{0}^{2}$ 

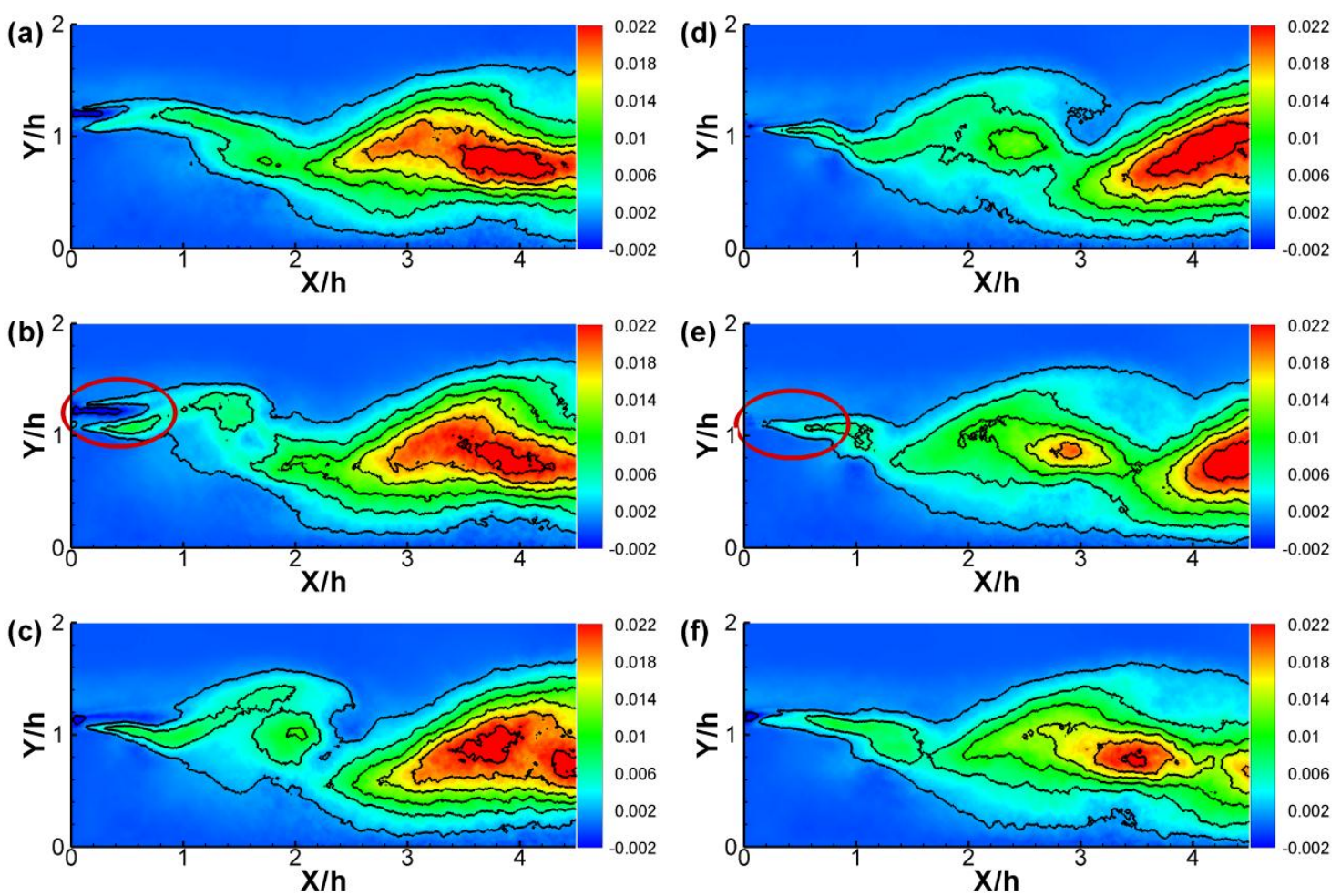

Figure 5- 4 Phase-averaged incoherent Reynolds shear stress $-\left\langle u^{\prime} \cdot v^{\prime}\right\rangle / U_{0}^{2}$ of the controlled case. (a)-(f) $\alpha=0^{\circ}, 60^{\circ}, 120^{\circ}, 180^{\circ}, 240^{\circ}, 300^{\circ}$
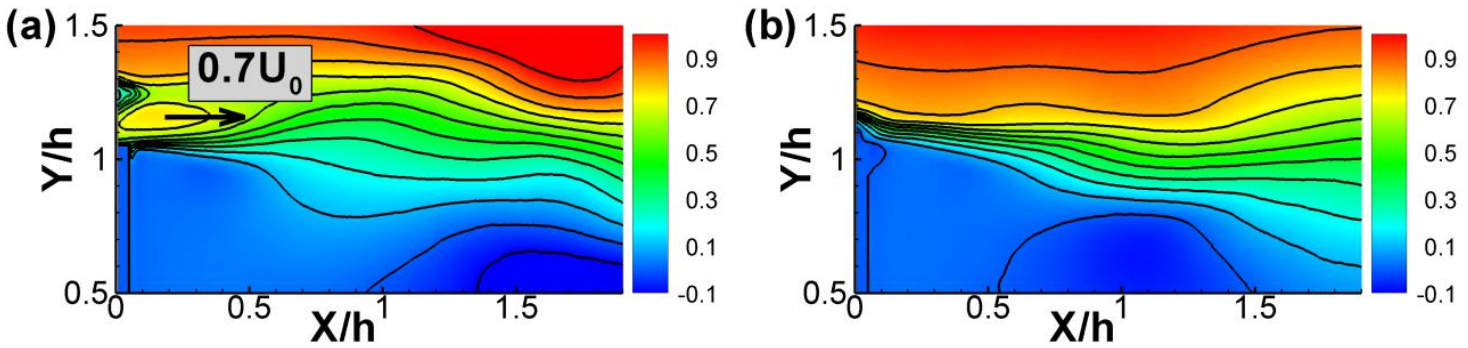

Figure 5- 5 Phase-averaged contours of streamwise velocity $-\langle\mathrm{u}\rangle / \mathrm{U}_{0}$. (a) $\alpha=60^{\circ}$; (b) $\alpha=240^{\circ}$

Figure 5- 4 shows the incoherent Reynolds shear stress that is obtained by the phase-locked velocity data. It is shown that the large-scale turbulent shear layer follows the small perturbations upwards and downwards as a flapping motion, which entrains the high momentum fluid into the shear layer. The vortex roll-up and breakdown contributes most to the part of the Reynolds shear stress in the shear layer. At the phase angle of $\alpha=60^{\circ}$, as the oscillating flap moves downward to the neutral position, it pushes the boundary layer and generates a small streamwise ejection with a velocity of $0.7 \cdot \mathrm{U}_{0}$ into the shear layer, as shown in Figure 5- 4(b) 
and Figure 5- 5. On the upper side of the ejection, there is a small additional shear layer which contains a negative velocity gradient $\partial\langle\mathrm{u}\rangle / \partial \mathrm{y}<0$ and negative coherent Reynolds shear stress $-\left\langle\mathrm{u}^{\prime} \cdot \mathrm{v}^{\prime}\right\rangle\langle 0$. This positive production of turbulence also satisfies the co-gradient momentum transport (Hussain 1986). On the other hand, at the opposite phase angle of $\alpha=240^{\circ}$, as the oscillating flap moves upwards to the neutral position, it pulls the separated shear layer upwards as well, as shown in Figure 5- 4(e). The flapping motion of the initial part of the shear layer exhibits identical frequency as the periodic small perturbations and generates two-dimensional waves with an estimated wavelength of $\Delta \mathrm{X} / \mathrm{h} \approx 1-2$.
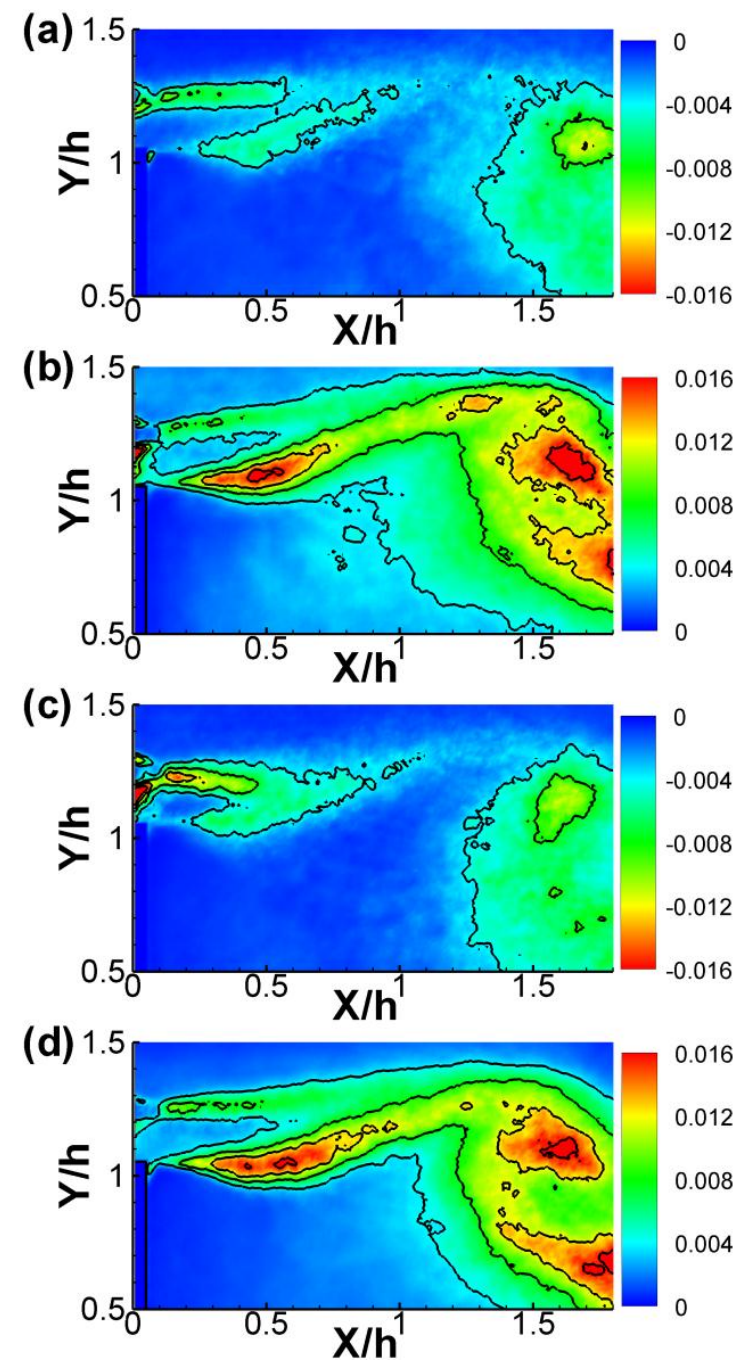
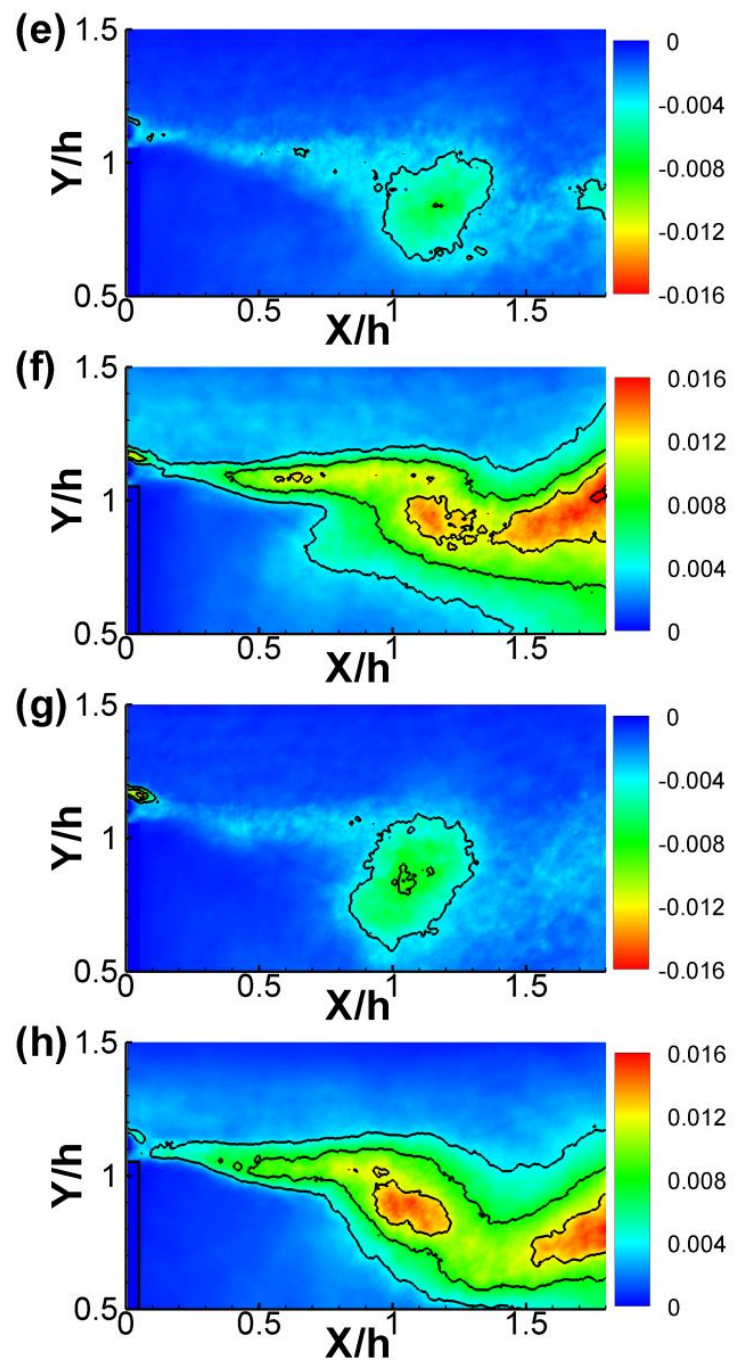

Figure 5- 6 Phase-averaged quadrant events $-\left\langle\mathrm{Q}_{\mathrm{i}}\right\rangle / \mathrm{U}_{0}^{2}$. (a)-(d) $\mathrm{Q}_{1}, \mathrm{Q}_{2}, \mathrm{Q}_{3}, \mathrm{Q}_{4}$ at $\alpha=60^{\circ}$; (e)-(h) $\mathrm{Q}_{1}, \mathrm{Q}_{2}$, $\mathrm{Q}_{3}, \mathrm{Q}_{4}$ at $\alpha=240^{\circ}$

Two typical phase-averaged Reynolds shear stress fields at $\alpha=60^{\circ}$ and $\alpha=240^{\circ}$ are further 
decomposed into four quadrant events and listed by column in Figure 5- 6. At $\alpha=60^{\circ}$, the $\mathrm{Q}_{1}$ and $\mathrm{Q}_{3}$ events have high values within the small shear layer on the upper side of the ejection whereas the $\mathrm{Q}_{2}$ and $\mathrm{Q}_{4}$ events play a predominant role in the main separated shear layer below the ejection. It is clear that the main separated shear layer follows the perturbations upwards and rolls up to a spanwise vortex at $\mathrm{X} / \mathrm{h} \approx 1.5$. This agrees well with the coherent Reynolds shear stress in Figure 5- 3(a). By contrast, the Figure 5- 6(e)-(h) show, when the oscillating flap is at the opposite phase position, the main separated shear layer contains mainly $\mathrm{Q}_{2}$ and $\mathrm{Q}_{4}$ events. Therefore, the phase-averaged quadrant analysis reveals the influence of the perturbations on the events of Reynolds shear stress as well as their spatial evolution. The small perturbations are able to conduct the initial part of the large-scale separated shear layer, which results in the flapping motions and quasi-periodic coherent structures further downstream.

\subsection{Spatial cross-correlation function}

Figure 5- 7 presents the spatial two-point cross-correlation functions of the vertical velocity component. The normalized coordinates of the reference points are $X_{0} / h=1.0,2.0,3.0$ and $Y_{0} / h$ = 1.0. In the clean case, as shown in Figure 5- 7(a-c), the elliptical regions of positive correlation is at the reference points of $\mathrm{X}_{0} / \mathrm{h}=1.0$ and two adjacent regions of negative correlation are locate upstream and downstream, though the negative correlation is weak. The rest part of the flow field shows little correlation feature due to the turbulent mixing motions. As the reference point moves downstream to $\mathrm{X}_{0} / \mathrm{h}=2.0$ and 3.0, the distance of the correlated regions increases. This is an indication that the spatial scale of the vortices within the shear layer increases as the vortices move downstream.

In the controlled case, a clear correlation pattern consisting of positive and negative correlation regions is shown in Figure 5- 7(d-f). The large-scale coherent structure has approximate one spatial period in the streamwise direction within $0<\mathrm{X} / \mathrm{h}<2$, which agrees well with the phase-averaged spanwise vortices in Figure 5- 4. In Figure 5- 7(f), the vortices are less-correlated as the reference point moves further downstream, which is mainly due to the 
attenuation of vortex strength, the in coherent turbulent mixing and the out-of-plane motions. Thus, the correlation results characterize the spatial scale and evolution of the coherent structures in the controlled case.


Figure 5- 7 Contours of coefficient of spatial cross-correlation. (a)-(c) $\mathrm{R}_{\mathrm{v}^{\prime} \mathrm{v}^{\prime}}$ of the clean case; (d)-(f) $R_{v^{\prime} v^{\prime}}$ of the controlled case. The reference points are at $Y_{0} / h=1.0$ and $X_{0} / h=1.0,2.0,3.0$, respectively.

\subsection{Structures and dynamics of the subharmonic waves}

In order to extract the large-scale spanwise vortices in the flapping motion of the shear layer, the snapshot POD method (Sirovich 1987) is applied in a rectangular region with a size of 3.3h $\times 1.3 \mathrm{~h}$ (Figure 5- 8), which focuses on the shear layer. In the present analysis, the number of the instantaneous velocity vector fields that are used is $\mathrm{N}=3,000$ and each snapshot contains 42,706 spatial points. 




Figure 5- 8 Sketch of the rectangular region of $3.3 \mathrm{~h} \times 1.3 \mathrm{~h}$ in which POD is applied

The POD eigenvalues of the clean and controlled cases are plotted in Figure 5- 9(a). It is shown that the first few POD modes contain high turbulent kinetic energy while the energy content of the other modes decays exponentially. The comparison of the clean and controlled cases shows that the turbulent fluctuating motions of the controlled case are larger than the clean case. The scatters of the coefficients reveal the coherent phase interrelations between the first two modes. Phase angle $\varphi_{1,2}$ of the scatter point $\left(\mathrm{a}_{1}, \mathrm{a}_{2}\right)$ are obtained as (Perrin et al. 2007):

$$
\varphi_{1,2}=\arctan \left(\frac{\sqrt{\lambda_{1}}}{\sqrt{\lambda_{2}}} \cdot \frac{a_{2}}{a_{1}}\right)
$$

The phase angles between the first two POD modes are determined directly from the velocity fields and thereby the phase-jitter effect is reduced. The coefficients of the two pairs of POD modes are scattered in Figure 5- 9(b). In the clean case, the coefficients $a_{1}$ and $a_{2}$ gather to the center as a disk, which indicates that the two modes are independent and incoherent to each other with respect to a phase relation. In the controlled case, on the other hand, the coefficients $a_{1}$ and $a_{2}$ are scattered as a circle, which shows the two modes are coherent and contain a fixed phase difference. The other modes of the controlled case do not show this coherent feature. 



Figure 5- 9 (a) POD eigenvalues of the first 100 modes; (b) Scatter distributions of POD coefficients
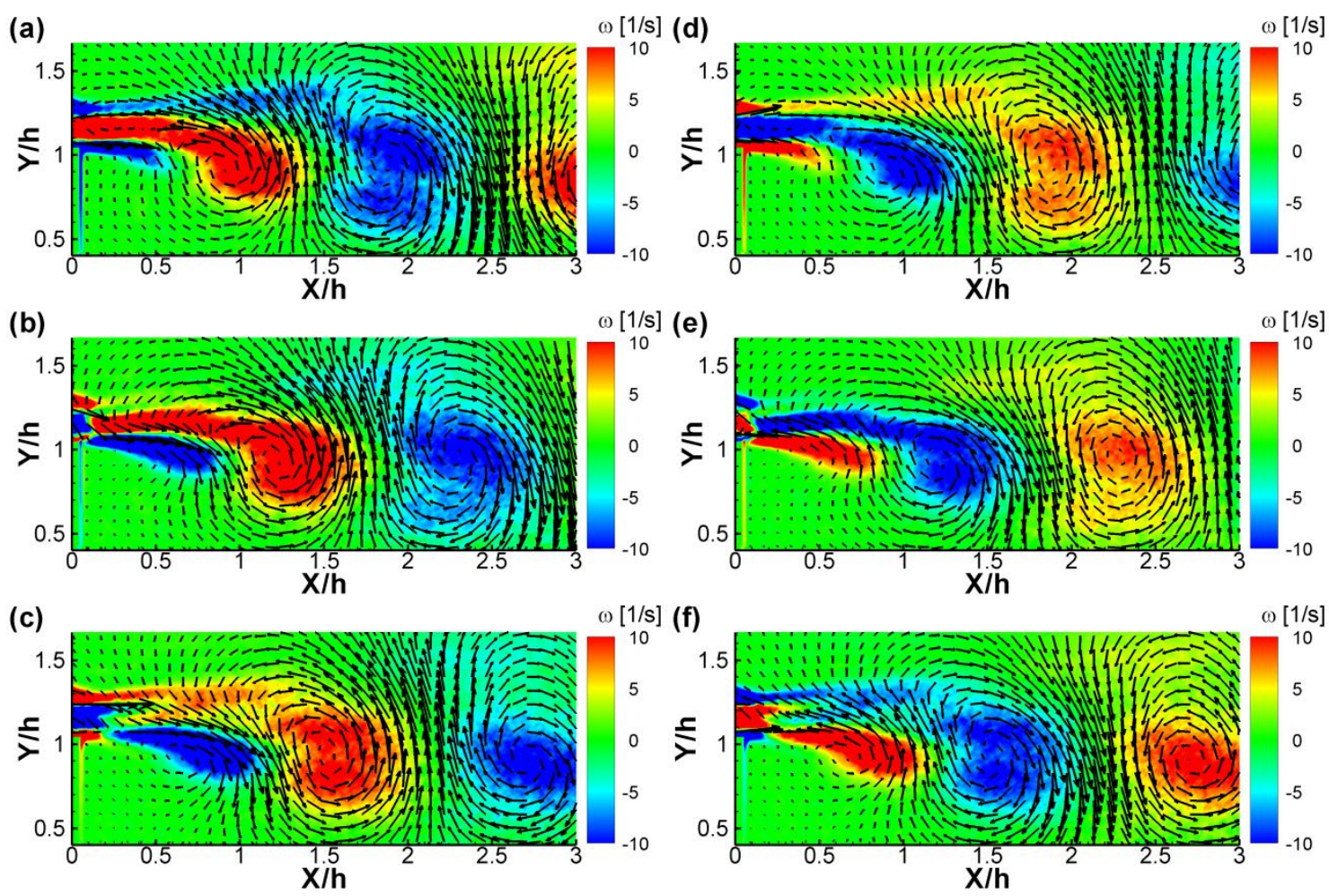

Figure 5- 10 POD Reconstruction by $\mathrm{POD}_{1}$ and $\mathrm{POD}_{2}$ of the controlled case. (a)-(f) $\varphi_{1,2}=0^{\circ}, 60^{\circ}, 120^{\circ}$, $180^{\circ}, 240^{\circ}, 300^{\circ}$. The color indicates spanwise vorticity. Every 5 th vector is shown for visual clarity.

POD reconstruction can be obtained by linearly combining the first two modes weighted by their coefficients. As shown in Figure 5- 10, large-scale counter-rotating vortices in the controlled case are shown. This regular pattern indicates the coherent feature between $\mathrm{POD}_{1}$ and 
$\mathrm{POD}_{2}$, which can be presented as a composite form "POD $+\mathrm{i} \cdot \mathrm{POD}_{2}$ " (Schmid et al. 2012). An estimated wavelength of the vortices is $\Delta \mathrm{X} / \mathrm{h} \approx 2$, which agrees well with the spatial scale in the phase-averaged Reynolds shear stress in Figure 5- 4. Thus, it can be concluded that the series of vortices are generated by the periodic small perturbations.

In order to analyze dynamic features of these coherent structures, the DMD method is applied to the POD reconstructed data containing $\mathrm{POD}_{1}$ and $\mathrm{POD}_{2}$ :

$$
\mathbf{V}_{\mathbf{x}}=\sum_{\mathrm{i}=1}^{\mathrm{i}=2} \mathbf{a}_{\mathbf{i}} \cdot \boldsymbol{\phi}_{\mathbf{i}}
$$

In other words, the POD decomposition and reconstruction act as a spatial filter by keeping large-scale flow structures and filtering small scales, incoherent turbulence and noise out.

(a)

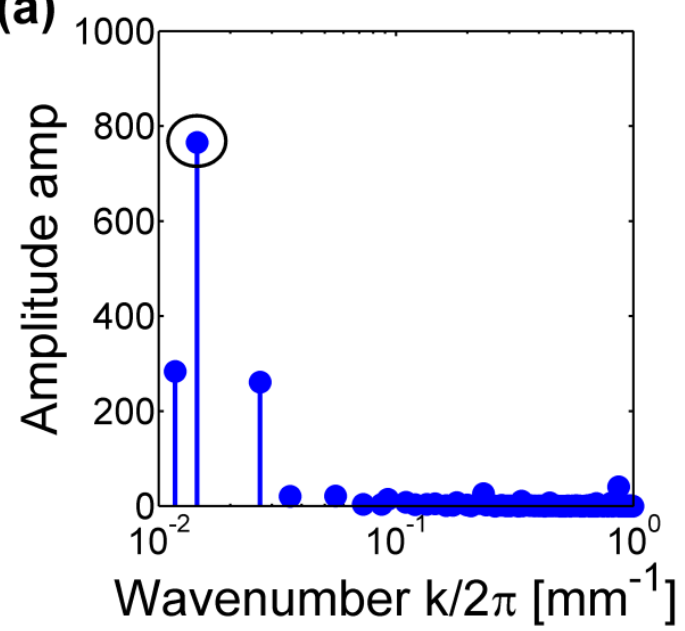

(b)

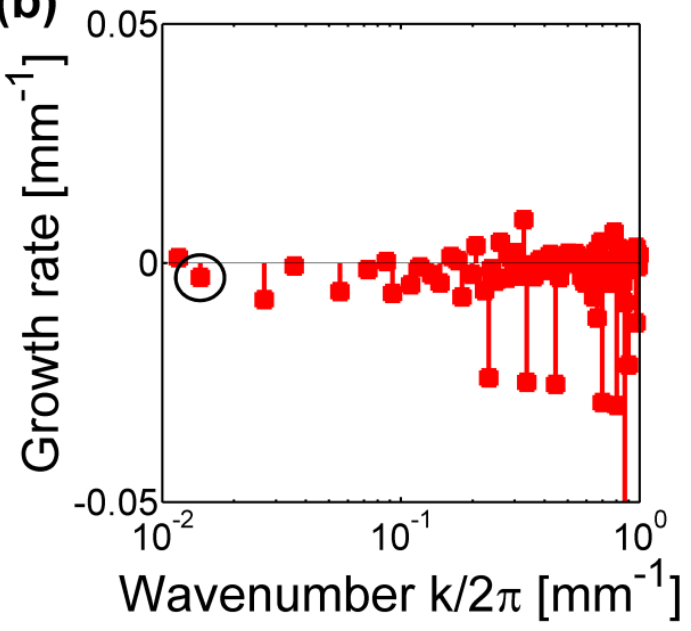

Figure 5- 11 (a) Spatial DMD amplitude in frequency doman; (b) DMD growth rate in frequency doman. The negative frequencies are omitted for clarity.

Figure 5- 11(a)-(b) show the absolute values of the amplitudes and spatial growth rates of the DMD modes in the wavenumber domain. The absolute values of the amplitudes measure the importance of each DMD mode: the predominant mode $\mathrm{DMD}_{6}$ has a complex frequency $\mathrm{f}_{6}=$ $(-0.003,0.0145) \mathrm{mm}^{-1}$ and has the highest amplitude in the spectrum, which is marked by a black ellipse. The real and imaginary parts of the complex frequency correspond to the spatial growth rate and wavenumber, respectively. Therefore, the predominant wave has a wavelength 
of $\Delta \mathrm{X} / \mathrm{h}=2.3(69.0 \mathrm{~mm})$ and decays as it travels downstream in the turbulent shear layer. This wavelength agrees well with the estimation of $\Delta \mathrm{X} / \mathrm{h} \approx 2$ in the POD reconstruction in Figure 510.

The DMD reconstruction is performed by the multiplication of the mode $\mathrm{DMD}_{6}$, the amplitude $\left|a_{6}\right|$ and the spatial evolution $e^{2 \pi \cdot f_{6} \cdot \Delta x}$, which leads to evolving flow structures in the streamwise direction (Figure 5- 12). Being different from the spatial orthogonality of POD, the spatial DMD reconstruction by the mode of $\mathrm{DMD}_{6}$ represents a single-wavenumber wave extracted from the quasi-periodic coherent structures, which is closely related to the periodic small perturbations.

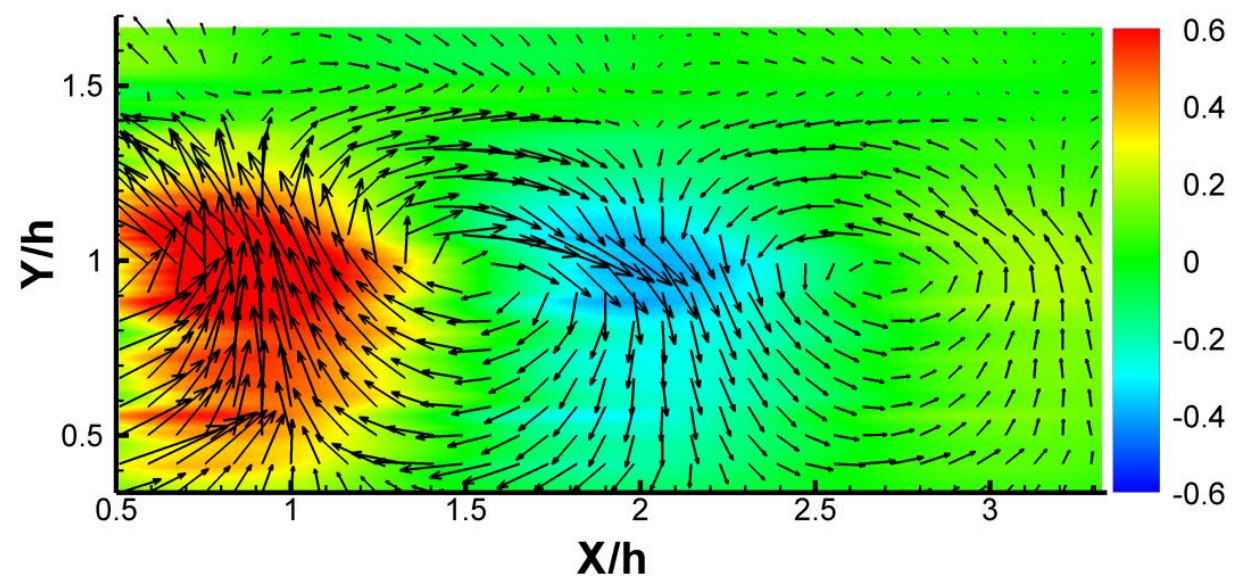

Figure 5- 12 Spatial DMD reconstruction by $\mathrm{DMD}_{6}$. The color indicates the vertical velocity component $\mathrm{v}[\mathrm{m} / \mathrm{s}]$.

In order to compare the amplitudes of the perturbations and the resulting waves, the vertical velocity component at the streamwise line of $\mathrm{Y} / \mathrm{h}=1$ is extracted from the phase-averaged, POD reconstructed and DMD reconstructed data (Figure 5- 13). The maximum vertical velocity of the perturbations is $0.86 \mathrm{~m} / \mathrm{s}\left(<0.09 \cdot \mathrm{U}_{0}\right)$, which is less than $0.09 \cdot \mathrm{U}_{0}$. However, the quasi-periodic waves are generated in the separated shear layer with an amplitude higher than $0.15 \cdot \mathrm{U}_{0}$ (represented by the black curve). By reducing the phase-jitter effect, the POD data shows a much clearer wave with an increasing amplitude and an increasing wavelength. The 
DMD data is a single-wavenumber component in the POD reconstructed data, which corresponds to the single-frequency perturbations. It is shown that this single-wavenumber wave has a relative small amplitude which decays quickly in this turbulent shear flow. However, the two-dimensional waves with considerable amplitudes are generated in the large-scale separated shear layer. The vortex structures and corresponding coherent features can be extracted by POD and DMD in a complementary way.

Therefore, some remarks of the oscillating flap are discussed. First, as an active flow control device, the oscillating flap is driven by vertical periodic Ampere force and generates small perturbations at the backward-facing step, resulting in a time-averaged reduction of the reattachment length by $31.0 \%$ downstream of the BFS. Second, the periodic small perturbations have a subharmonic frequency, which is half of the most-amplified frequency of the shear layer. The subharmonic perturbations are able to effectively manipulate the large-scale separated shear layer and generated quasi-periodic coherent structures downstream. Third, compared with the harmonic actuations of the acoustic tube, the subharmonic perturbations lead to the flapping motion of the shear layer without vortex merging.

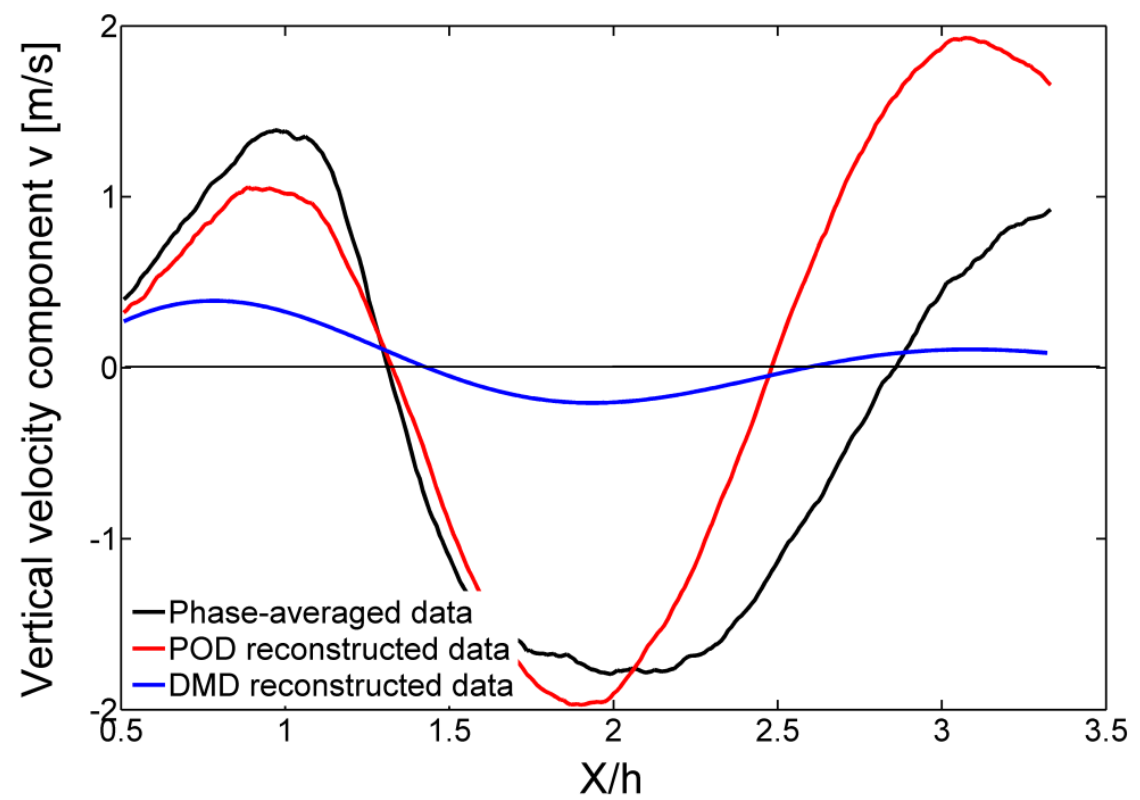

Figure 5-13 Spatial developments of vertical velocity components $v[\mathrm{~m} / \mathrm{s}]$ at the line of $\mathrm{Y} / \mathrm{h}=1$ 
"If the whole problem appears mathematically hopeless, see what happens if an essential parameter of the problem approaches zero."

Ludwig Prandtl (1875-1953) 


\section{Results of vortex generators}

The four VG configurations as well as the clean BFS have been tested independently by high-resolution 2D-2C PIV and time-resolved tomographic PIV. In the three-dimensional measurement, 3,000 instantaneous velocity vector fields have been obtained at a sampling frequency of $1 \mathrm{kHz}$ in each case. The time-averaged streamwise velocity profiles, within the shear layer at $\mathrm{X} / \mathrm{h}=1, \mathrm{Y} / \mathrm{h}=-0.5,0$ and 0.5 and $0.8<\mathrm{Z} / \mathrm{h}<1.2$, are plotted in Figure 6- 1. It is shown that there is a two-dimensional mean flow in the clean case. On the other hand, the controlled case $(\mathrm{H}=10 \mathrm{~mm}$, backward orientation) exhibits spanwise variations of the velocity profiles due to three-dimensional flow. The mean flow directly downstream of the center VG was accelerated by approximately $0.3 \cdot \mathrm{U}_{0}$ and the vertical velocity gradient was increased correspondingly, especially at the center line of $\mathrm{Y} / \mathrm{h}=0$. The "common flow" between the counter-rotating streamwise vortex pair was discussed by Mehta et al. (1988), which leads to a change of the mean flow due to the flow induction of the vortices. It is also found that there is a slight difference of approximately $0.01 \cdot \mathrm{U}_{0}$ between the velocity profiles of $\mathrm{Y} / \mathrm{h}= \pm 0.5$ downstream of the VGs. The spanwise asymmetry will be discussed in detail in the following analysis. 


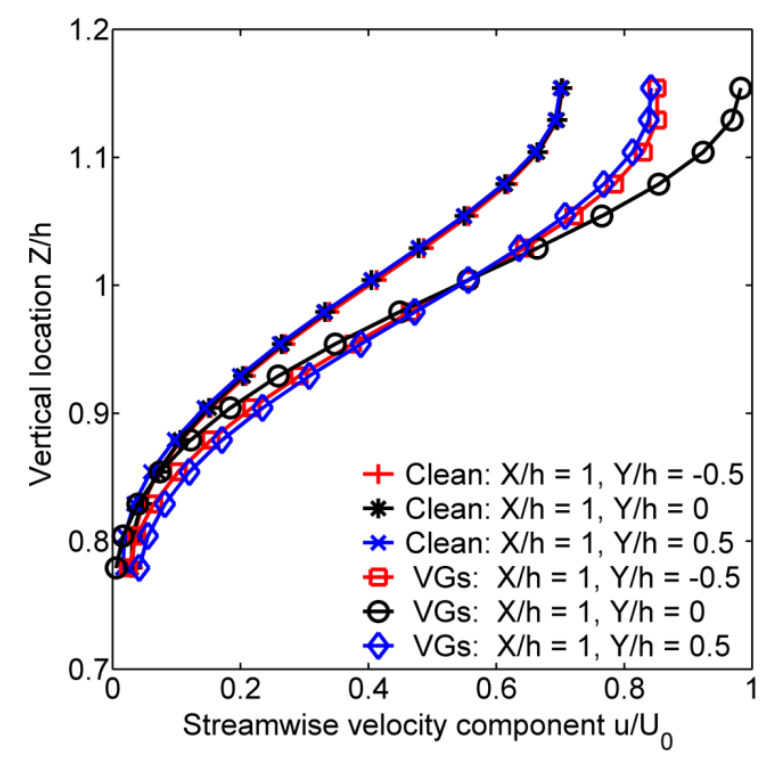

Figure 6- 1 Streamwise mean velocity profiles in the turbulent shear layer

\subsection{Parameter study and flow control result}

The time-averaged velocity profile and fluctuating velocities of the boundary layer are shown in

Figure 6- 2. It is clear that the present VGs are completely submerged within the turbulent boundary layer where high velocity gradients and strong velocity fluctuations are present. Although the velocity gradient and fluctuations may be underestimated due to the limitation of the PIV spatial resolution, the streamwise velocity fluctuation shows a peak close to the wall and the vertical velocity fluctuation is constrained by the wall, which is comparable with the classical zero-pressure-gradient turbulent boundary layer measurement by hot-wire anemometry by Klebanoff (1955).

In the parameter study, four VG configurations with two VG heights $(\mathrm{H}=10$ or $5 \mathrm{~mm})$ and two orientations (backward or forward) are tested independently. The other parameters, such as length and spanwise spacing, are kept constant in all VG test cases. The parameter sets and resulting reattachment lengths are listed in Table 6- 1. Among the four configurations, the case "VG-H10b" results in an apparent reduction of reattachment length by $29.1 \%$, which is better than the other VG configurations. The time-averaged velocity vector fields of the four VG cases 
are compared in Figure 6- 3. It is shown that the backward-oriented VGs have more reduction of the reattachment length than the forward-oriented ones. On the other hand, the VGs with the height of $\mathrm{H}=10 \mathrm{~mm}$ introduce stronger influence into the shear layer and produce higher drag values than those of $\mathrm{H}=5 \mathrm{~mm}$. The shear layer is accelerated in the streamwise direction and pulled downward closer to the wall by the configuration of "VG-H10b" in Figure 6- 3(a), while it is decelerated and pulled up clearly by the configuration of "VG-H10f" in Figure 6- 3(c). Thus, as the most effective configuration, the case "VG-H10b" is denoted as "controlled case" and the clean BFS flow is denoted as "clean case" for further analysis.
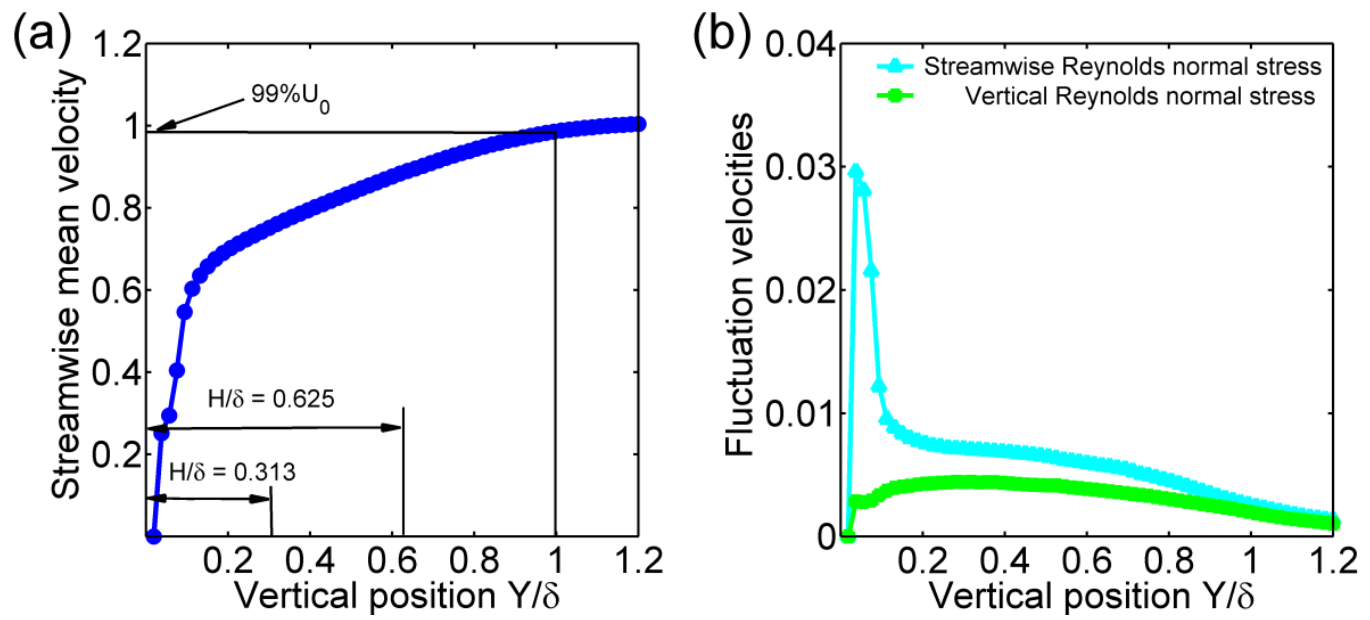

Figure 6- 2 Time-averaged velocity profile and fluctuations of incoming turbulent boundary layer. (a) streamwise velocity profile $\overline{\mathrm{u}} / \mathrm{U}_{0}$; (b) Reynolds normal stress $\overline{\mathrm{u}^{\prime} \mathrm{u}^{\prime}} / \mathrm{U}_{0}^{2}, \overline{\mathrm{v}^{\prime} \mathrm{v}^{\prime}} / \mathrm{U}_{0}^{2}$

Table 6- 1 Reduction of reattachment length of different VG configurations

\begin{tabular}{lllllllll}
\hline \hline Case & $\mathrm{H} / \delta$ & $\mathrm{e} / \mathrm{H}$ & $\beta\left[^{\circ}\right]$ & $\Delta \mathrm{Y} / \mathrm{H}$ & $\Delta \mathrm{X} / \mathrm{H}$ & Orientation & $\mathrm{L} / \mathrm{h}$ & Label \\
\hline clean BFS & $/$ & $/$ & $/$ & $/$ & $/$ & $/$ & 7.1 & clean case \\
VG-H10b & 0.625 & 10 & 14 & 6 & 10 & backward & $\mathbf{5 . 0 3}$ & controlled case \\
VG-H05b & 0.3125 & 10 & 14 & 6 & 10 & backward & 6.1 & $/$ \\
VG-H10f & 0.625 & 10 & 14 & 6 & 10 & forward & 6.33 & $/$ \\
VG-H05f & 0.3125 & 10 & 14 & 6 & 10 & forward & 6.43 & $/$ \\
\hline \hline
\end{tabular}



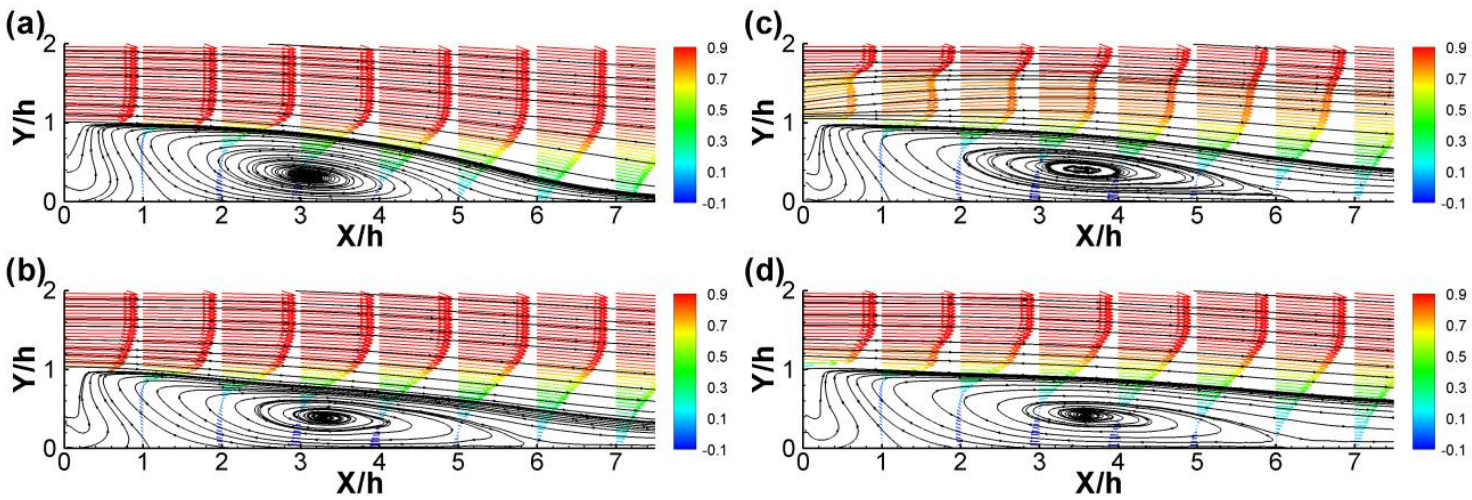

Figure 6- 3 Time-averaged velocity vector fields of the four VG configurations. (a) $\mathrm{H}=10 \mathrm{~mm}$, backward; (b) $\mathrm{H}=5 \mathrm{~mm}$, backward; (c) $\mathrm{H}=10 \mathrm{~mm}$, forward; (d) $\mathrm{H}=5 \mathrm{~mm}$, forward. The color indicates streamwise velocity component $\overline{\mathrm{u}} / \mathrm{U}_{0}$.
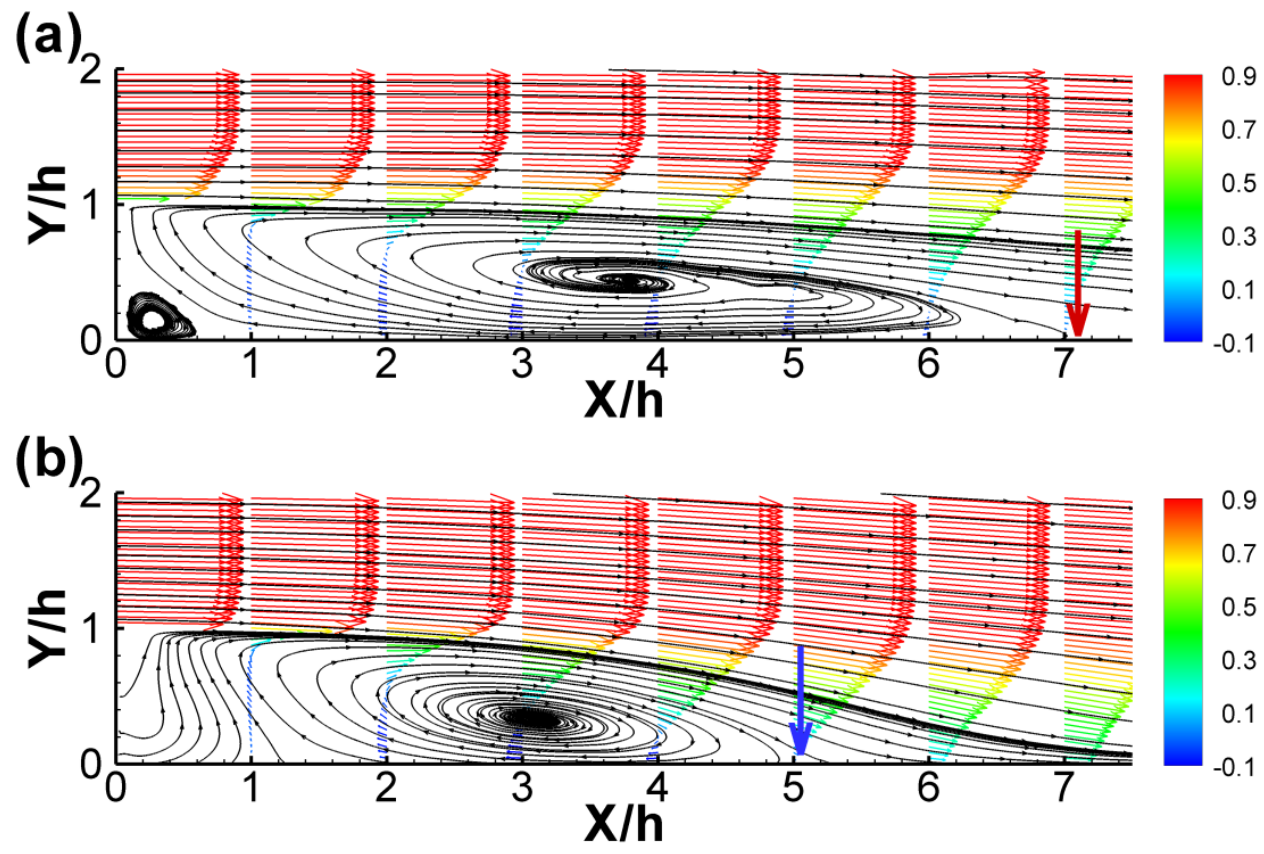

Figure 6- 4 Comparison of time-averaged velocity vector fields. (a) clean case; (b) controlled case. The arrows indicate the reattachment points. The color indicates streamwise velocity component $\overline{\mathrm{u}} / \mathrm{U}_{0}$.

Time-averaged velocity vector fields of the clean and controlled cases are compared in Figure 6- 4. In the clean case, the main and the secondary recirculation regions are clearly identified behind the step and the reattachment length is $\mathrm{L}_{0} / \mathrm{h}=7.1$. In the controlled case, the streamlines are drawn downward closer to the wall. The size of the main recirculation region is clearly reduced and its center position moves upstream. The reattachment length is reduced to $L_{c} / h=$ 
5.03 , resulting in a reduction rate of $\mathrm{R}_{\mathrm{L}}=29.1 \%$. It is noted that the secondary recirculation region disappears and the time-averaged streamlines start from the step wall, as shown in Figure 6- 4(b). One reason for the three-dimensional spanwise mean flow is that the VG-induced vortices could entrain neighboring fluid into the low-pressure low-speed recirculation region.

The time-averaged and instantaneous three-dimensional velocity vector fields of the clean and controlled cases are shown in Figure 6- 5. The iso-surfaces of normalized vorticity magnitude $|\omega|^{*}=|\omega| \cdot h / U_{0}$ show the classical two-dimensional spanwise vortices in the clean BFS flow whereas for the controlled case the $\Lambda$-shape vorticity profile and accelerated velocity region is identified downstream of the VGs. Moreover, the comparison of the instantaneous snapshots indicates that more vortex structures are generated downstream of the VGs.
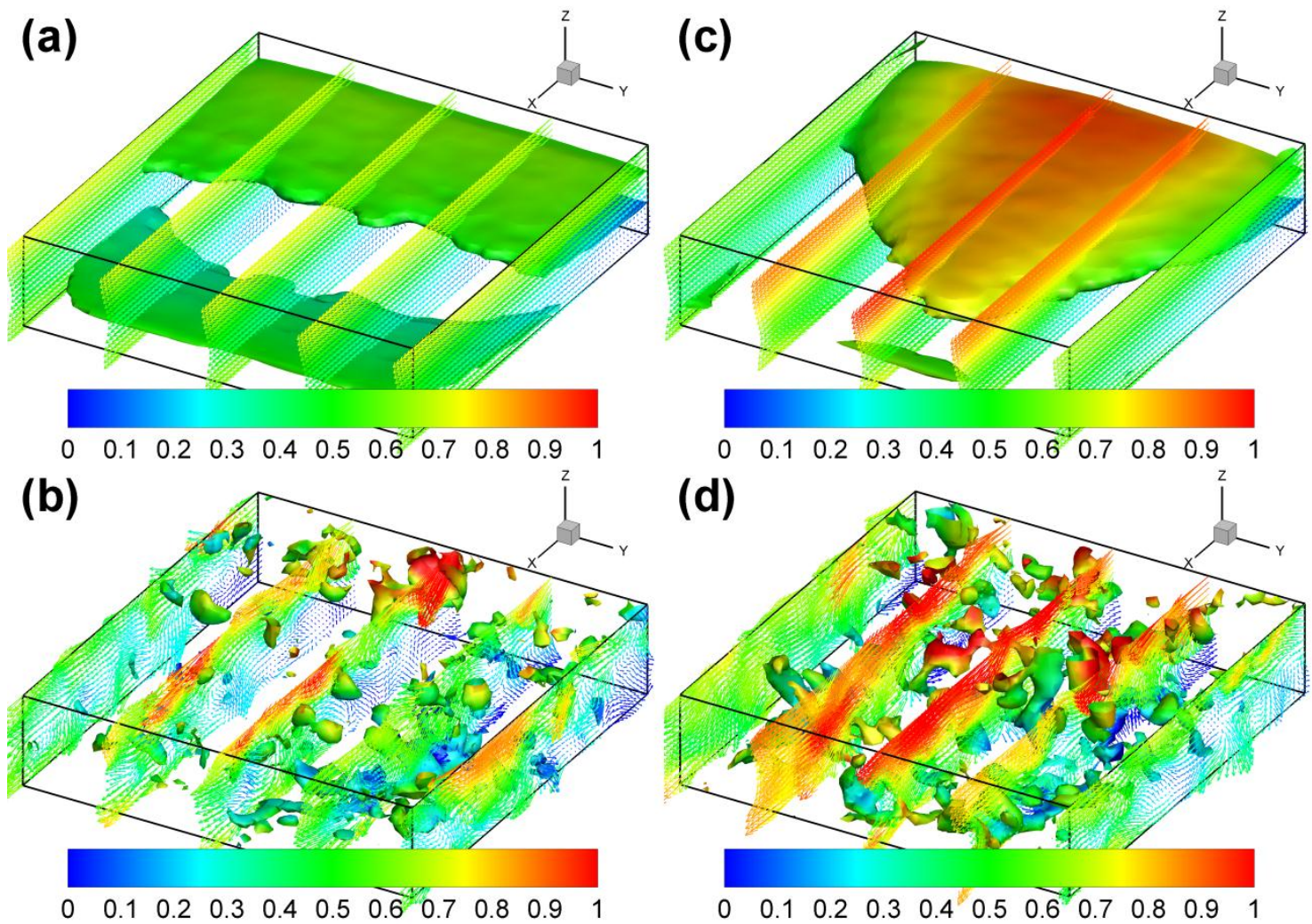

Figure 6- 5 (a) time-averaged velocity vector field of the clean case with the iso-surfaces of $|\omega|^{*}=2,250 ;(\mathbf{b})$ instantaneous velocity vector field of the clean case with the iso-surfaces of $|\omega|^{*}=8,400$; (c) time-averaged velocity vector field of the controlled case with the iso-surfaces of $|\omega|^{*}=2,250$; (d) instantaneous velocity 
vector field of the controlled case with the iso-surfaces of $|\omega|^{*}=8,400$. The incoming flow is in the X direction. The vectors and iso-surfaces are color-coded by streamwise velocity component $\bar{u} / U_{0}$.

However, there is hardly any apparent regularity in the vortex structures downstream of the VGs, as the large-scale vortices cascade down to the small-scale ones at the high Reynolds number. Therefore, the VG-induced vortices are strongly influenced by turbulent mixing and therefore are easily broken apart, leaving only irregular pieces.

\subsection{Analysis of Reynolds shear stress}

Reynolds shear stress consisting of the streamwise and vertical velocity components reveals the fluctuating fluid motions and momentum transfer in the turbulent shear layer. The comparison between the clean and controlled cases shows a considerable increase of Reynolds shear stress downstream of the VGs (Figure 6- 6). The high-value region locates in the center of the volume right behind the VG and agrees well with the time-averaged $\Lambda$-shape vorticity profile in Figure 6- 5(c). Therefore, the increased Reynolds shear stress is directly related to the interaction of the VG-induced vortices.
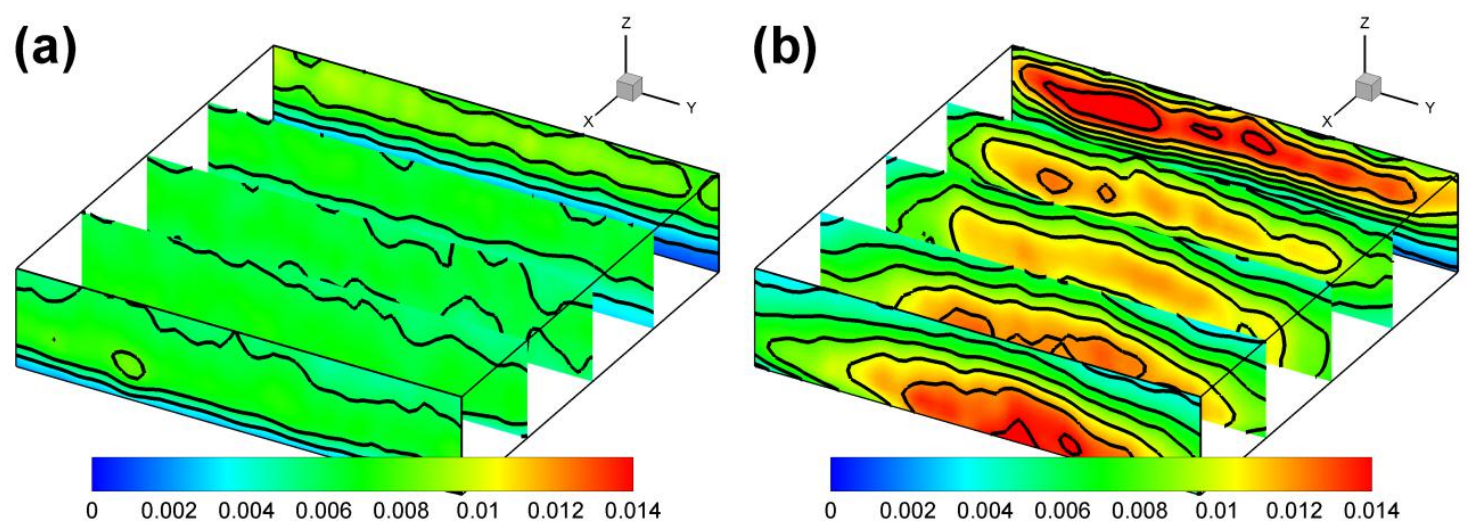

Figure 6- 6 Comparison of Reynolds shear stress $-\overline{\mathrm{u}^{\prime} \mathrm{w}^{\prime}} / \mathrm{U}_{0}^{2}$. (a) clean case; (b) controlled case. The incoming flow is in the $\mathrm{X}$-direction.

Further comparison among the four VG configurations addresses the resulting Reynolds shear stress of the different VG configurations. Although the two parameters, the heights and 
orientations, both influence the flow fields downstream, the VGs of $\mathrm{H}=10 \mathrm{~mm}$ result in higher increase of the Reynolds shear stress in the shear layer than those of $\mathrm{H}=5 \mathrm{~mm}$, as compared between Figure 6- 7(a) and (b). On the other hand, the backward-oriented VGs produce the counter-rotating vortices which are closer to each other and result in greater vortex decay than those of forward-orientation, as shown in Figure 6- 7(a) and (c). This result agrees well with the experiment carried out by Ashill et al. (2001), who explained the stronger vortex decay downstream of the backward wedge VGs is due to the stronger influence of the wall shear flow, because the VG-induced vortices are closer to the wall than other types of VGs.
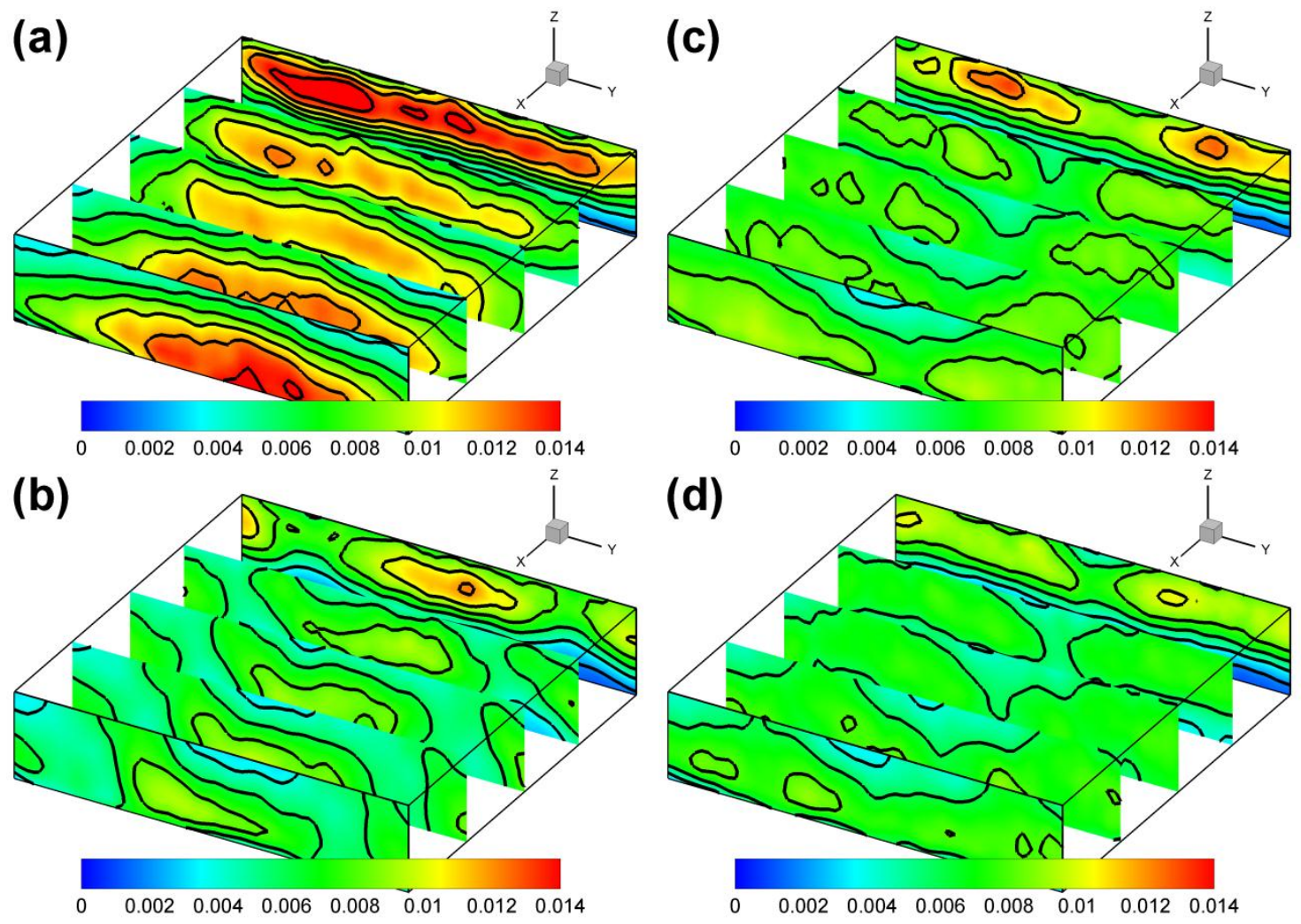

Figure 6- 7 Reynolds shear stress $-\overline{\mathrm{u}^{\prime} \mathrm{w}^{\prime}} / \mathrm{U}_{0}^{2}$ of the four VG configurations. (a) $\mathrm{H}=10 \mathrm{~mm}$, backward; (b) $\mathrm{H}=5 \mathrm{~mm}$, backward; (c) $\mathrm{H}=10 \mathrm{~mm}$, forward; (d) $\mathrm{H}=5 \mathrm{~mm}$, forward. The incoming flow is in the $\mathrm{X}$ direction.

\subsection{Spatial and temporal cross-correlation functions}

Spatial and temporal cross-correlation functions are applied to the three-dimensional flow field 
in order to characterize the structure and dynamic features of the VG-induced coherent structures. Figure 6- 8 shows the comparisons of the spatial cross-correlations of the clean and controlled cases. The normalized coordinate of the reference point is $\left(\mathrm{X}_{0} / \mathrm{h}, \mathrm{Y}_{0} / \mathrm{h}, \mathrm{Z}_{0} / \mathrm{h}\right)=(1.3$, $0.0,1.0)$. In the clean case, the ellipsoidal region of positive correlation (red) locates near the reference point where it is equivalent to its auto-correlation function. Two adjacent regions of negative correlation of $\overline{u^{\prime} \cdot u^{\prime}}$ (blue) are aligned in the spanwise direction in Figure 6- 8(a), and another two adjacent regions of negative correlation of $\overline{\mathrm{w}^{\prime} \cdot \mathrm{w}^{\prime}}$ are aligned in the streamwise direction in Figure 6- 8(c). Four adjacent regions of negative correlation of $\overline{v^{\prime} \cdot v^{\prime}}$ clearly surround the reference point and are inclined toward the free-stream flow in Figure 68(b). The inclined correlated structures were described by Townsend (1970) as the "inclined double-roller structure" for a general shear flow. In the controlled case, by contrast, the fluctuating velocities appear to be positive-correlated in the streamwise direction, which implies an underlying large-scale quasi-periodic structure downstream of the VGs.

Temporal evolution of the cross-correlated flow structures within a time duration of $5 \mathrm{~ms}$ are shown in Figure 6- 9, Figure 6- 10 and Figure 6- 11, consisting of $\overline{u^{\prime}(t) \cdot u^{\prime}(t+d t)}$, $\overline{v^{\prime}(t) \cdot v^{\prime}(t+d t)}$ and $\overline{w^{\prime}(t) \cdot w^{\prime}(t+d t)}$, respectively. The reference point is at $\left(X_{0} / h, Y_{0} / h\right.$, $\left.\mathrm{Z}_{0} / \mathrm{h}\right)=(1.0,0.0,1.0)$. At the first instant of time of $\mathrm{dt}=0 \mathrm{~ms}$, the temporal cross-correlation is equivalent to the spatial cross-correlation at the reference point. As the flow structures move downstream, the peaks of the cross-correlations move downstream as well and decrease gradually due to the mixing motions with the surrounding turbulent fluid (Favre et al. 1957). The present streamwise convection velocity of the turbulent structures in the center of the measurement volume can be estimated to be $\mathrm{U}_{\mathrm{c}} \approx 0.38 \cdot \mathrm{U}_{0}$, which is close to the local mean velocity of the shear layer. This estimation agrees well with the experimental results of convection velocities in the shear layer of a circular jet, which were measured by Wills (1964). He found that the longitudinal convection velocity is equal to the local longitudinal mean velocity downstream of the separation edge. Moreover, the convection velocities are slightly lower than local mean velocities on the high-speed side of the shear layer and slightly higher 
than local mean velocities on the low-speed side (Wills 1964).

\section{clean}

(a)

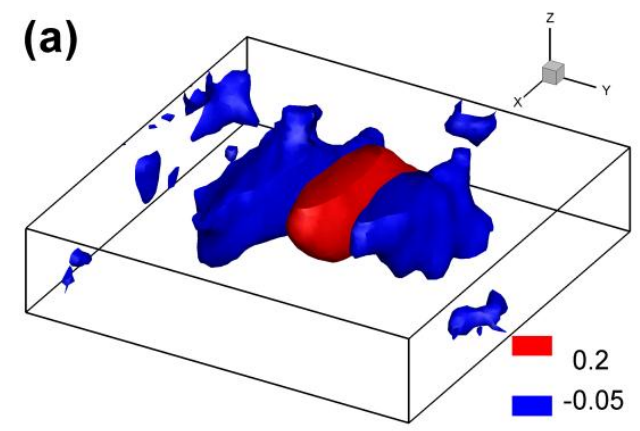

(b)



(c)

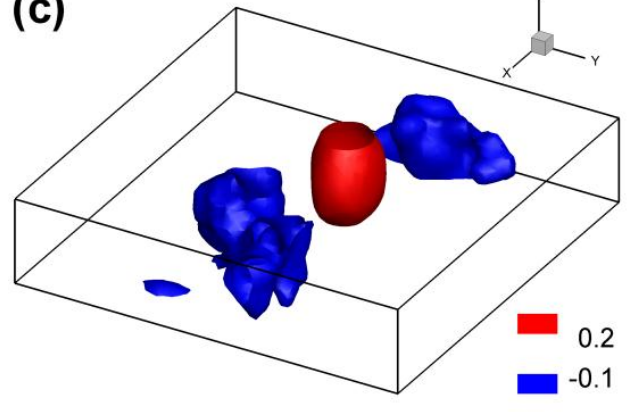

\section{controlled}
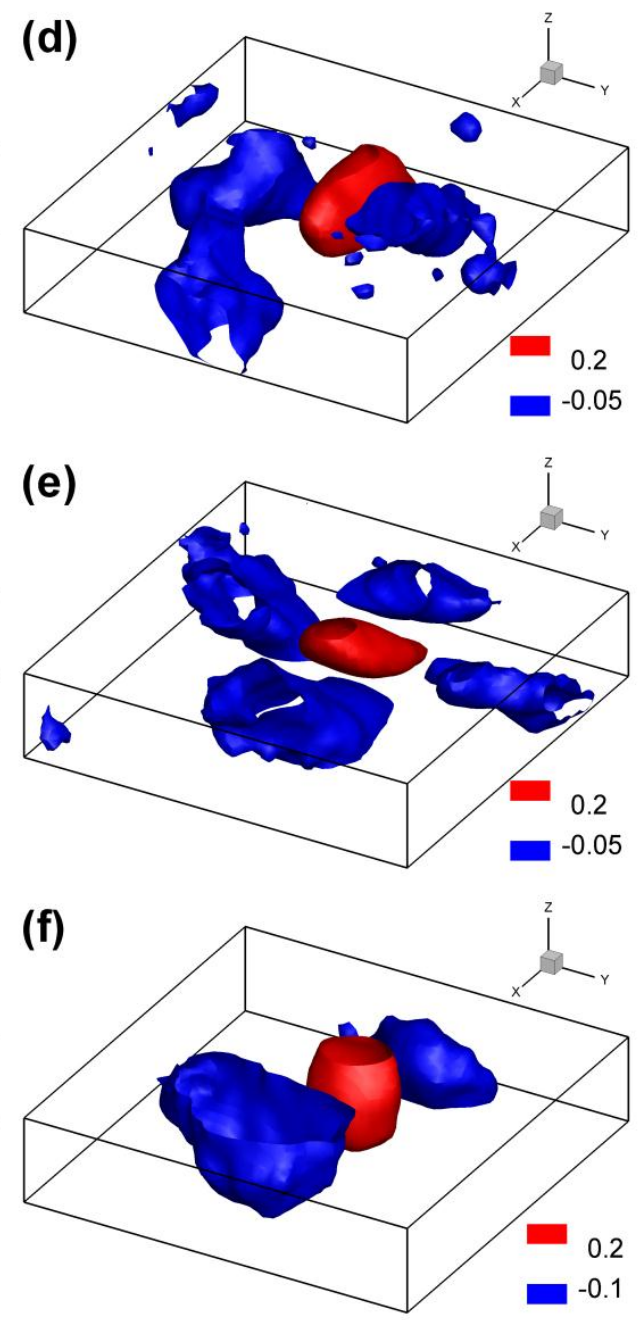

Figure 6- 8 Iso-surfaces of coefficient of spatial cross-correlation with the reference point at $(1.3,0.0,1.0)$. (a)-(c) $\mathrm{R}_{\mathrm{u}^{\prime} \mathrm{u}^{\prime}}, \mathrm{R}_{\mathrm{v}^{\prime} \mathrm{v}^{\prime}}$ and $\mathrm{R}_{\mathrm{w}^{\prime} \mathrm{w}^{\prime}}$ of the clean case; (d)-(f) $\mathrm{R}_{\mathrm{u}^{\prime} \mathrm{u}^{\prime}}, \mathrm{R}_{\mathrm{v}^{\prime} \mathrm{v}^{\prime}}$ and $\mathrm{R}_{\mathrm{w}^{\prime} \mathrm{w}^{\prime}}$ of the controlled case. 



$$
\mathbf{t}=\mathbf{2} \text { [ms] }
$$


$$
\mathbf{t}=\mathbf{3} \text { [ms] }
$$
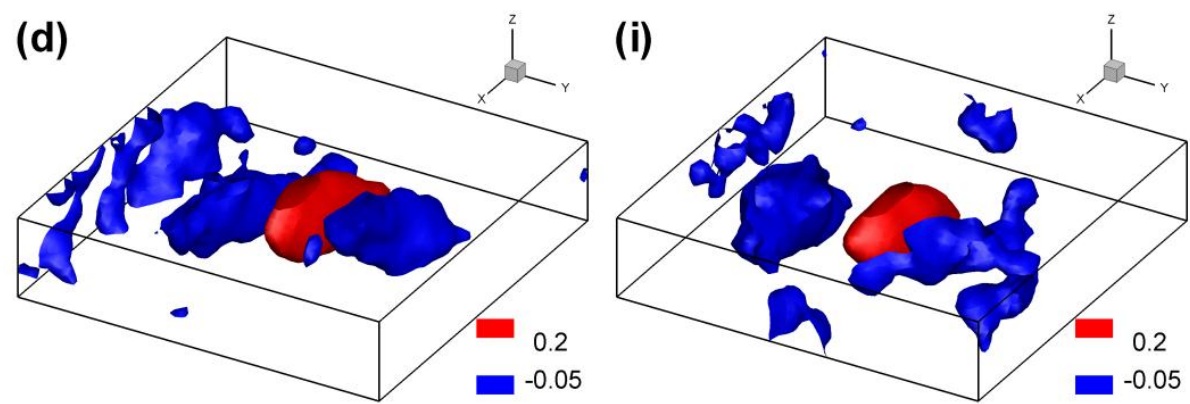

$\mathrm{t}=4[\mathrm{~ms}]$
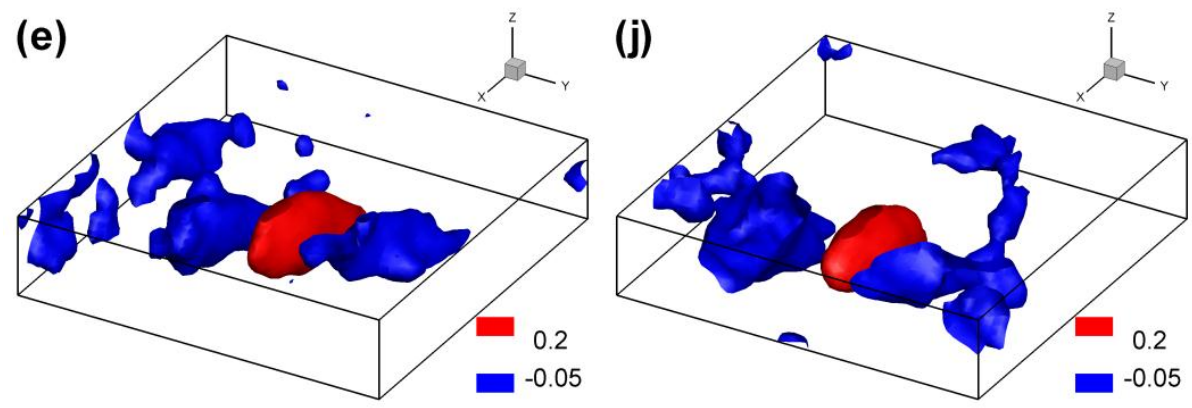

\section{$\mathbf{t}=\mathbf{5}[\mathrm{ms}]$}

Figure 6- 9 Iso-surfaces of coefficient of temporal cross-correlation at $t=1,2, \ldots, 5 \mathrm{~ms}$ with the reference point at $(1.3,0.0,1.0)$. (a)-(e) $\mathrm{R}_{\mathrm{u}^{\prime} \mathrm{u}^{\prime}}(\mathrm{t})$ of the clean case; (f)-(j) $\mathrm{R}_{\mathrm{u}^{\prime} \mathrm{u}^{\prime}}(\mathrm{t})$ of controlled case. 



$\mathbf{t}=\mathbf{2}[\mathrm{ms}]$


$\mathbf{t}=\mathbf{3}[\mathrm{ms}]$
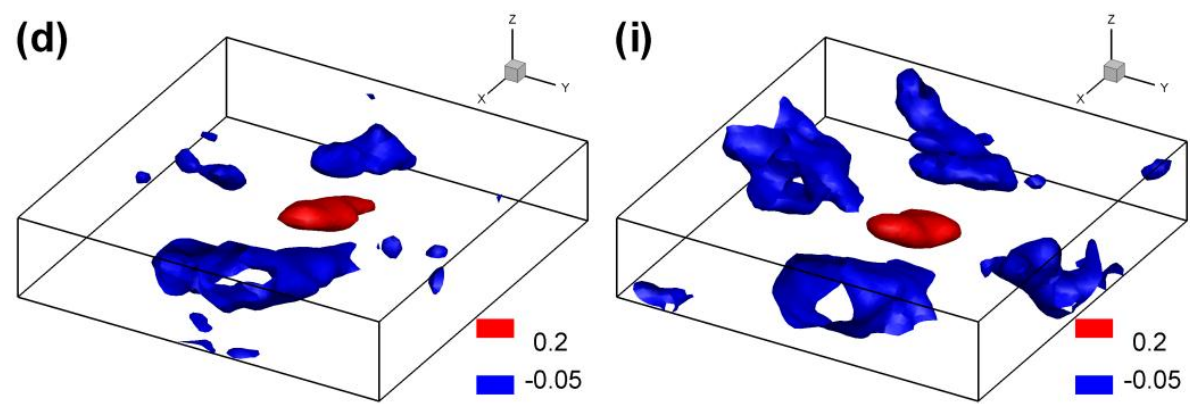

\section{$\mathrm{t}=\mathbf{4}[\mathrm{ms}]$}
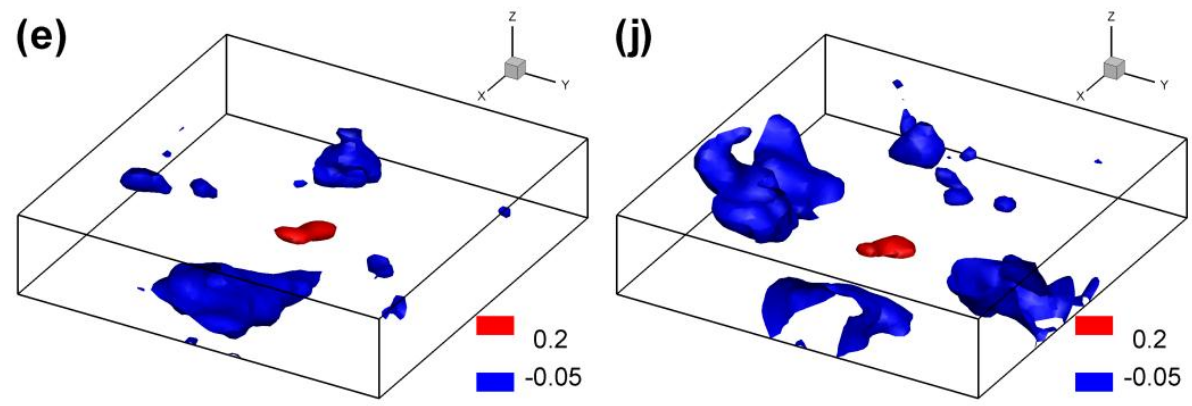

\section{$\mathbf{t}=\mathbf{5}[\mathrm{ms}]$}

Figure 6- 10 Iso-surfaces of coefficient of temporal cross-correlation at $\mathrm{t}=1,2, \ldots, 5 \mathrm{~ms}$ with the reference point at $(1.3,0.0,1.0)$. (a)-(e) $R_{v^{\prime} v^{\prime}}(t)$ of the clean case; (f)-(j) $R_{v^{\prime} v^{\prime}}(t)$ of controlled case. 

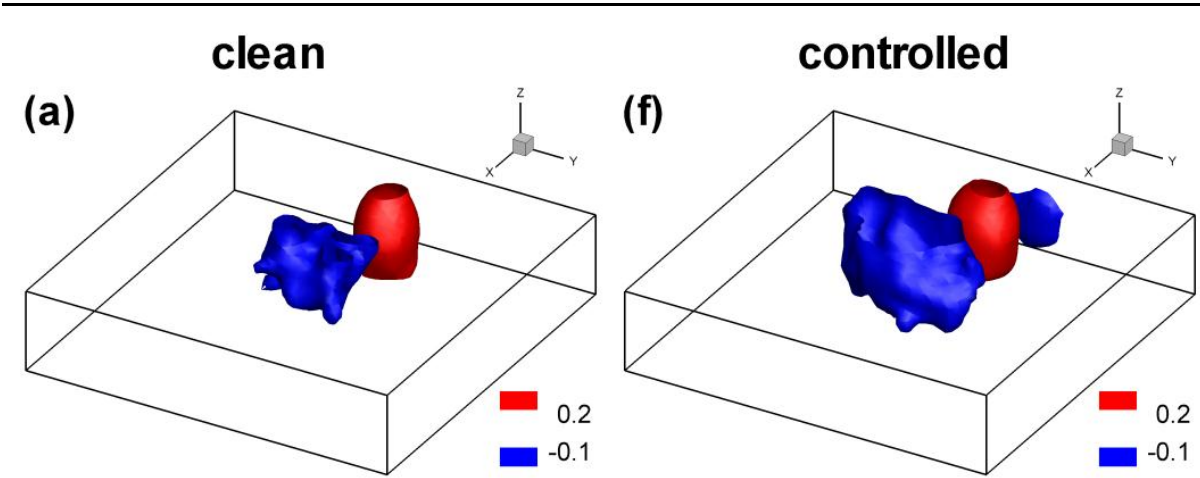

$$
\mathrm{t}=1 \text { [ms] }
$$
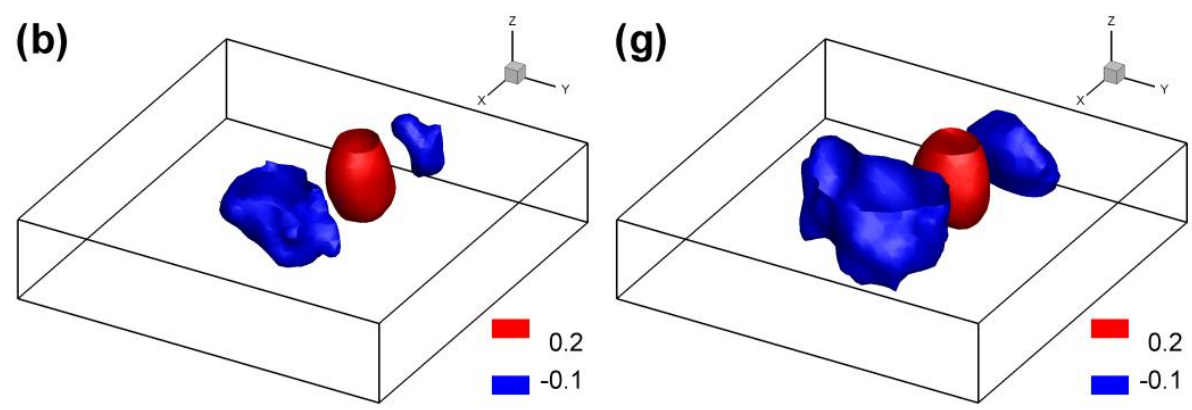

$$
\mathrm{t}=2 \text { [ms] }
$$


$$
\mathbf{t}=\mathbf{3}[\mathrm{ms}]
$$
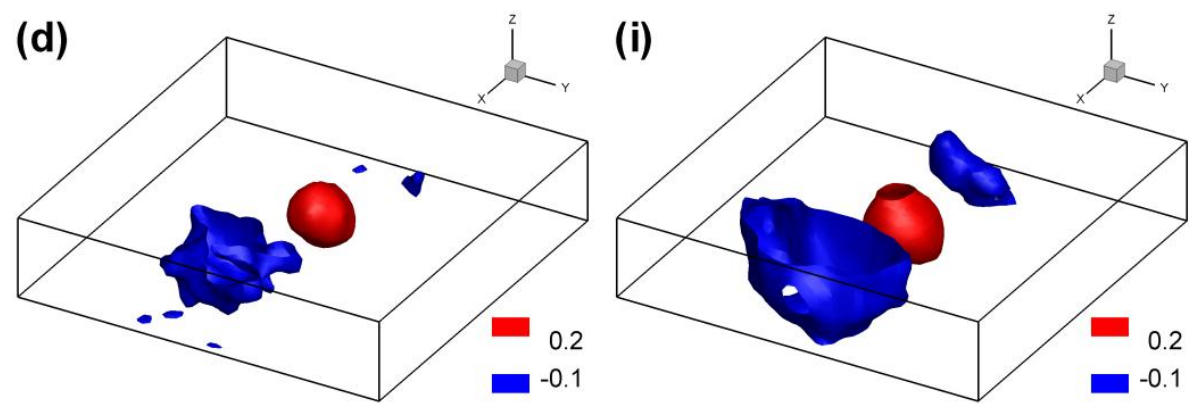

\section{$\mathrm{t}=4[\mathrm{~ms}]$}
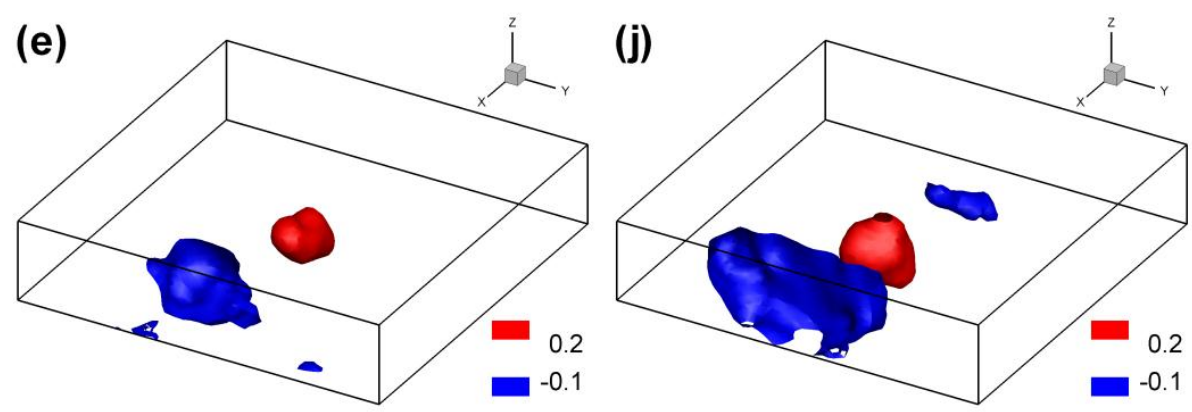

\section{$\mathbf{t}=\mathbf{5}[\mathrm{ms}]$}

Figure 6- 11 Iso-surfaces of coefficient of temporal cross-correlation at $\mathrm{t}=1,2, \ldots, 5 \mathrm{~ms}$ with the reference point at $(1.3,0.0,1.0)$. (a)-(e) $\mathrm{R}_{\mathrm{w}^{\prime} \mathrm{w}^{\prime}}(\mathrm{t})$ of the clean case; (f)-(j) $\mathrm{R}_{\mathrm{w}^{\prime} \mathrm{w}^{\prime}}(\mathrm{t})$ of controlled case. 


\subsection{Analysis of three-dimensional coherent structures}

\subsubsection{POD analysis}

In this part, snapshot POD is applied to decompose the original data sequence into a series of spatially orthogonal modes. The POD eigenvalue distributions are plotted in Figure 6- 12. It can be seen that the first few eigenvalues of the controlled case are higher than those of the clean case, which indicates that more energetic fluctuating motions are generated by the VGs. In both cases, the first few modes contain the major part of fluctuating energy and the further modes decay exponentially due to the descending energy-ranking.



Figure 6- 12 POD eigenvalue distributions of the first 100 modes

As marked by two blue ellipses in Figure 6- 12, the first two modes $\mathrm{POD}_{1}$ and $\mathrm{POD}_{2}$ of the controlled case contain the same order of turbulent kinetic energy, and so do the following modes $\mathrm{POD}_{3}$ and $\mathrm{POD}_{4}$. The coefficients $\mathrm{a}_{1}$ and $\mathrm{a}_{2}$ are plotted in Figure 6- $\mathbf{1 3}$ as time-evolving amplitudes of the normalized modes. Although being mutually uncorrelated, these two coefficients have similar frequencies and a nearly fixed phase difference, which implies underlying quasi-periodic coherent features. Then the statistical quantity "coherence" is used to examine the interrelation between the two modes in the frequency domain, which is defined as: 


$$
\mathrm{C}_{12}=\frac{\left|\mathrm{P}_{12}\right|^{2}}{\mathrm{P}_{11} \cdot \mathrm{P}_{22}}
$$

$\mathrm{P}_{11}$ and $\mathrm{P}_{22}$ are the auto-spectral density functions of the coefficients $\mathrm{a}_{1}$ and $\mathrm{a}_{2}$, and $\mathrm{P}_{12}$ is the cross-spectral density function of them, which are calculated by the Welch's method (Welch 1967). The spectrum has a frequency range up to $500 \mathrm{~Hz}$ as the Nyquist frequency of the sampling frequency. The resulting coherence $C_{12}$ has non-negative values in the range of $0 \leq C_{12}$ $\leq 1$. If $\mathrm{C}_{12}=0$, the two modes are incoherent; If $\mathrm{C}_{12}=1$, they are completely coherent; otherwise they are partially coherent. In the present study, normalized frequency or Strouhal number is defined as $S t_{h}=f \cdot h / U_{0}$, based on the free-stream velocity and the step height. It is revealed in Figure 6- 14 that $\mathrm{POD}_{1}$ and $\mathrm{POD}_{2}$ are highly coherent $\left(\mathrm{C}_{12}>0.95\right)$ in the particular frequency bandwidth of $0.3<\mathrm{St}_{\mathrm{h}}<0.6(100<\mathrm{f}<200 \mathrm{~Hz})$, outside the two modes are much less coherent or are governed by random fluctuations. Thus, "coherent bandwidth" is used to characterize a special frequency bandwidth, other than a single value, in which the fluid motions exhibit apparent coherent features. This phenomenon was also discussed by Bhattacharjee et al. (1986), who measured the velocity power spectrum of a BFS separated shear layer at $\operatorname{Re}_{\mathrm{h}}=$ $4.5 \times 10^{4}$ and found a broad bandwidth of $0.2<\mathrm{St}_{\mathrm{h}}<0.6$, other than a distinct peak in the spectrum. Besides the first two modes, the following modes $\mathrm{POD}_{3}$ and $\mathrm{POD}_{4}$ contain the similar coherent bandwidth in the spectrum as well (Figure 6- 15). The coherent feature are also identified in the bandwidth of $0.3<\mathrm{St}_{\mathrm{h}}<0.6$, although it is not as high as $\mathrm{POD}_{1}$ and $\mathrm{POD}_{2}$. 


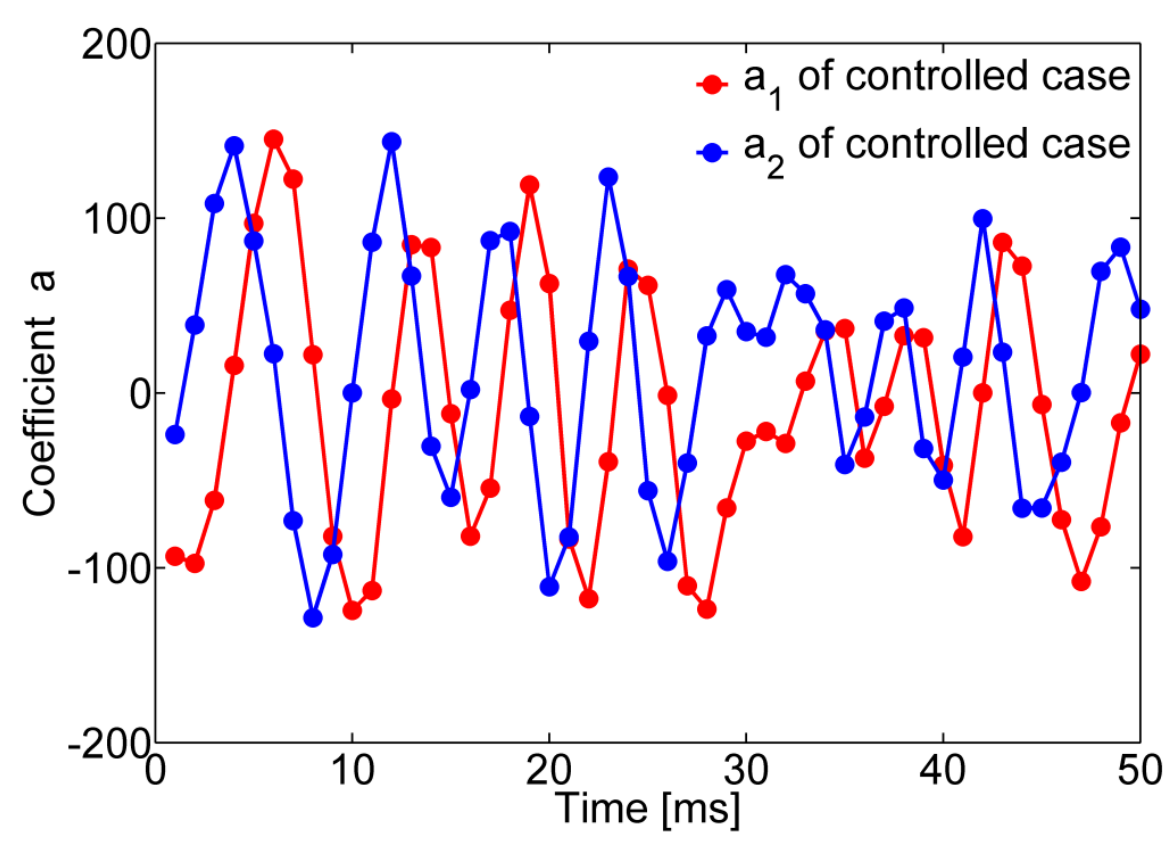

Figure 6- 13 Temporal evolutions of the coefficients of $\mathrm{POD}_{1}$ and $\mathrm{POD}_{2}$



Figure 6- 14 Coherence of the coefficients of $\mathrm{POD}_{1}$ and $\mathrm{POD}_{2}$ 




Figure 6- 15 Coherence of the coefficients of $\mathrm{POD}_{3}$ and $\mathrm{POD}_{4}$

POD reconstruction consisting of the first two modes $\mathrm{POD}_{1}$ and $\mathrm{POD}_{2}$ is equivalent to a reduced-order approximation of the original data. Temporal evolutions of the reconstructed flow fields are shown in Figure 6- 16 and the vortices are depicted by iso-surface of the normalized streamwise vorticity $\omega_{\mathrm{x}}{ }^{*}=\omega_{\mathrm{x}} \cdot \mathrm{h} / \mathrm{U}_{0}$. It is identified that quasi-periodic oblique vortices travel downstream through the volume and are aligned in the positive- $\mathrm{X}$ and positive-Y orientation. The positive and negative streamwise vortices are marked by "A" and "B" in Figure 6- 16(a). There are mainly two reasons why the vortices are aligned in the streamwise-spanwise direction. One reason is that the strong streamwise vortices induce apparent mean spanwise flow in the turbulent shear layer, which can be seen in the accelerated velocity profiles downstream of the VGs in Figure 6- 1. The other reason is the superposition of the VG-induced streamwise vortices on the classical spanwise two-dimensional vortices downstream of the BFS. The vortex structures have a period of approximately $8 \sim 9 \mathrm{~ms}$, which corresponds to an approximate frequency of $111 \sim 125 \mathrm{~Hz}$. This estimation agrees well with the above-discussed spectral coherent bandwidth between $\mathrm{POD}_{1}$ and $\mathrm{POD}_{2}$ in Figure 6- 14. In order to reveal the whole view of the vortex structures, further analysis of the POD modes $\mathrm{POD}_{3}$ and $\mathrm{POD}_{4}$ is needed. 
In reconstruction by $\mathrm{POD}_{3}$ and $\mathrm{POD}_{4}$ (Figure 6- 17), similar quasi-periodic oblique vortices travel through the volume and are aligned in the positive- $\mathrm{X}$ and negative-Y orientation, which are comparative to the reconstruction by $\mathrm{POD}_{1}$ and $\mathrm{POD}_{2}$. The positive and negative streamwise vortices are marked by "C" and "D" in Figure 6- 17(a). It can be seen that the series of vortices "C" and " $\mathrm{D}$ " have nearly a comparable wavelength and period with the other series of vortices "A" and "B" in Figure 6- 16, but the two series of vortices have opposite inclinations. Because of containing less turbulent kinetic energy, the vortices " $C$ " and " $D$ " have lower vortex strength and smaller extents of vorticity. Thus, it can be inferred that the POD reconstructions by "POD $+\mathrm{POD}_{2}$ " and "POD $+\mathrm{POD}_{4}$ " reveal parts of the asymmetric $\Lambda$-shaped vortex structures downstream of the VGs from the energy point of view. The corresponding coherent feature can also be presented in the interrelation of the POD coefficients.

Figure 6- 18 presents that the Reynolds shear stress is phase-correlated with the vortices that are reconstructed by " $\mathrm{POD}_{1}+\mathrm{POD}_{2}+\mathrm{POD}_{3}+\mathrm{POD}_{4}$ ". All the time-resolved instantaneous snapshots are sorted into 12 equal-interval phases, which are determined by the coefficients of the $\mathrm{POD}_{1}$ and $\mathrm{POD}_{2}$ as (Perrin et al. 2007)

$$
\varphi_{1,2}=\arctan \left(\frac{\sqrt{\lambda_{1}}}{\sqrt{\lambda_{2}}} \cdot \frac{a_{2}}{a_{1}}\right)
$$

The phase information is directly obtained from the flow fields other than external signals (Ma et al. 2015). It is shown that the high Reynolds shear stress (cyan) is located between the shedding vortices and phase-correlated with the streamwise vorticity: the low-speed flow is induced upwards in the vertical direction and the high-speed flow is induced downwards as well by the vortex structures, which results in high momentum transfer parallel to the mean velocity gradient within the shear layer. Thus, the VG-induced $\Lambda$-shaped vortex structures are present as coherent motions and lead to an apparent increase of the momentum transfer in the shear layer.

As discussed in the mathematical algorithm, the POD method is based on the energy hierarchy of flow structures and focuses on the most energetic motions. Each POD mode is a mutually 
orthogonal basis in space other than in time. In other words, the POD modes as well as the resulting reconstructions contain multiple frequencies. Thus, there may be coherent motions within the coherent bandwidth which are contained in higher POD modes which are less energetic but also important.
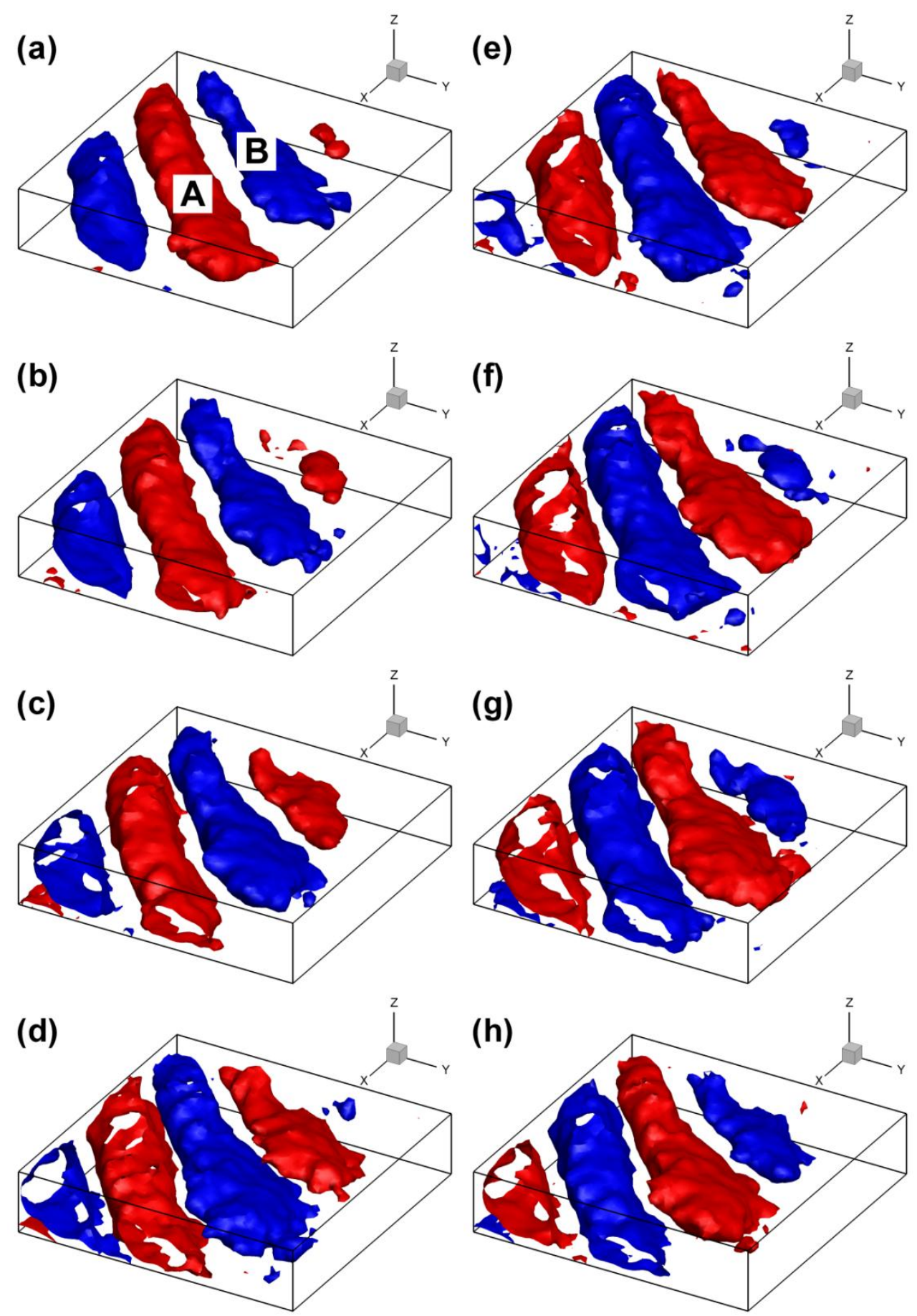

Figure 6- 16 Temporal evolutions of reconstruction by $\mathrm{POD}_{1}$ and $\mathrm{POD}_{2}$. (a)-(h) $\mathrm{t}=1,2, \ldots, 8$ ms. Positive (red) and negative (blue) iso-surfaces indicate normalized streamwise vorticity $\omega_{\mathrm{x}}{ }^{*}=\omega_{\mathrm{x}} \cdot \mathrm{h} / \mathrm{U}_{0}= \pm 0.3$. 

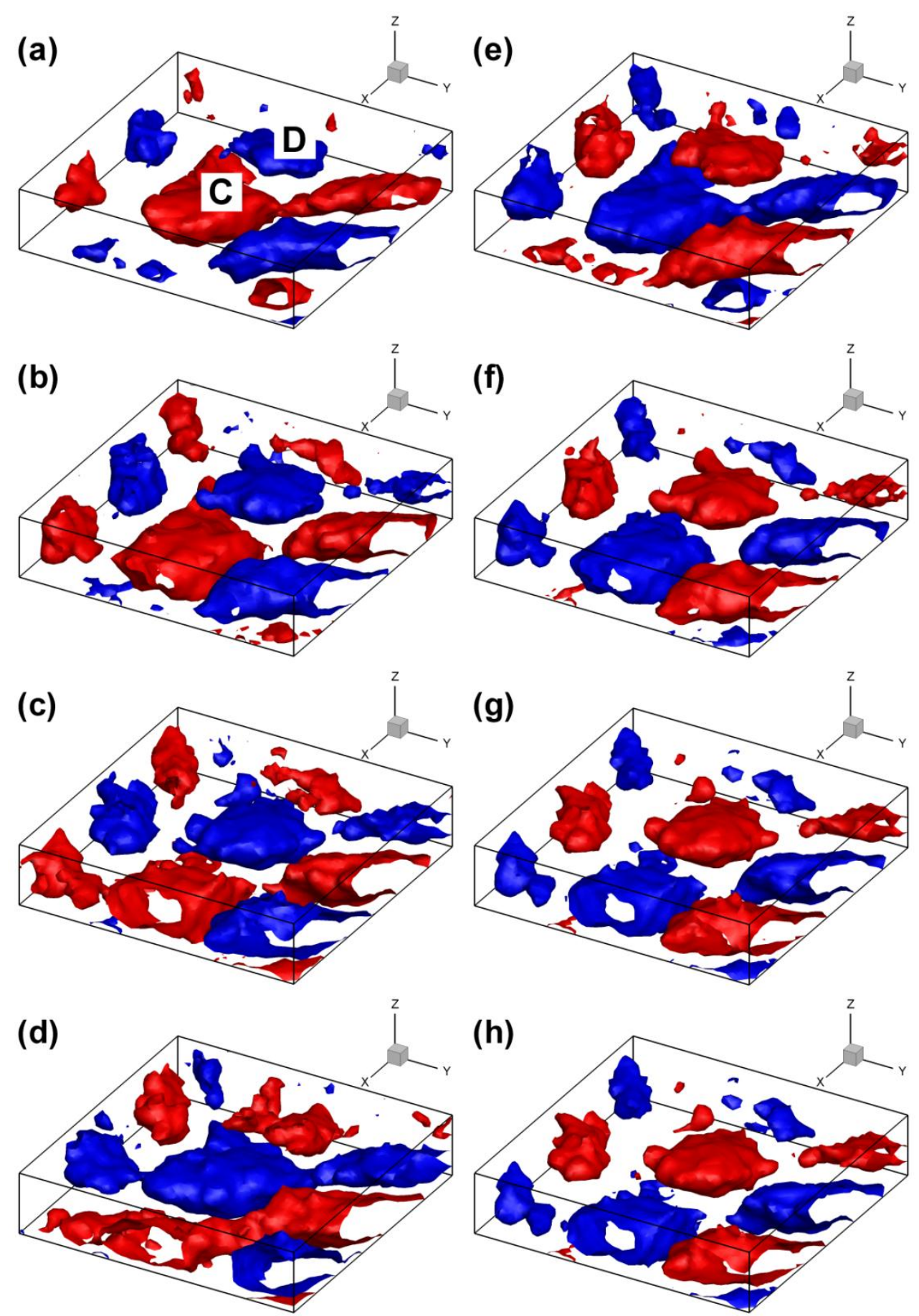

Figure 6- 17 Temporal evolutions of reconstruction by $\mathrm{POD}_{3}$ and $\mathrm{POD}_{4}$. (a)-(h) $\mathrm{t}=1,2, \ldots, 8$ ms. Positive (red) and negative (blue) iso-surfaces indicate normalized streamwise vorticity $\omega_{\mathrm{x}}{ }^{*}=\omega_{\mathrm{x}} \cdot \mathrm{h} / \mathrm{U}_{0}= \pm 0.3$. 

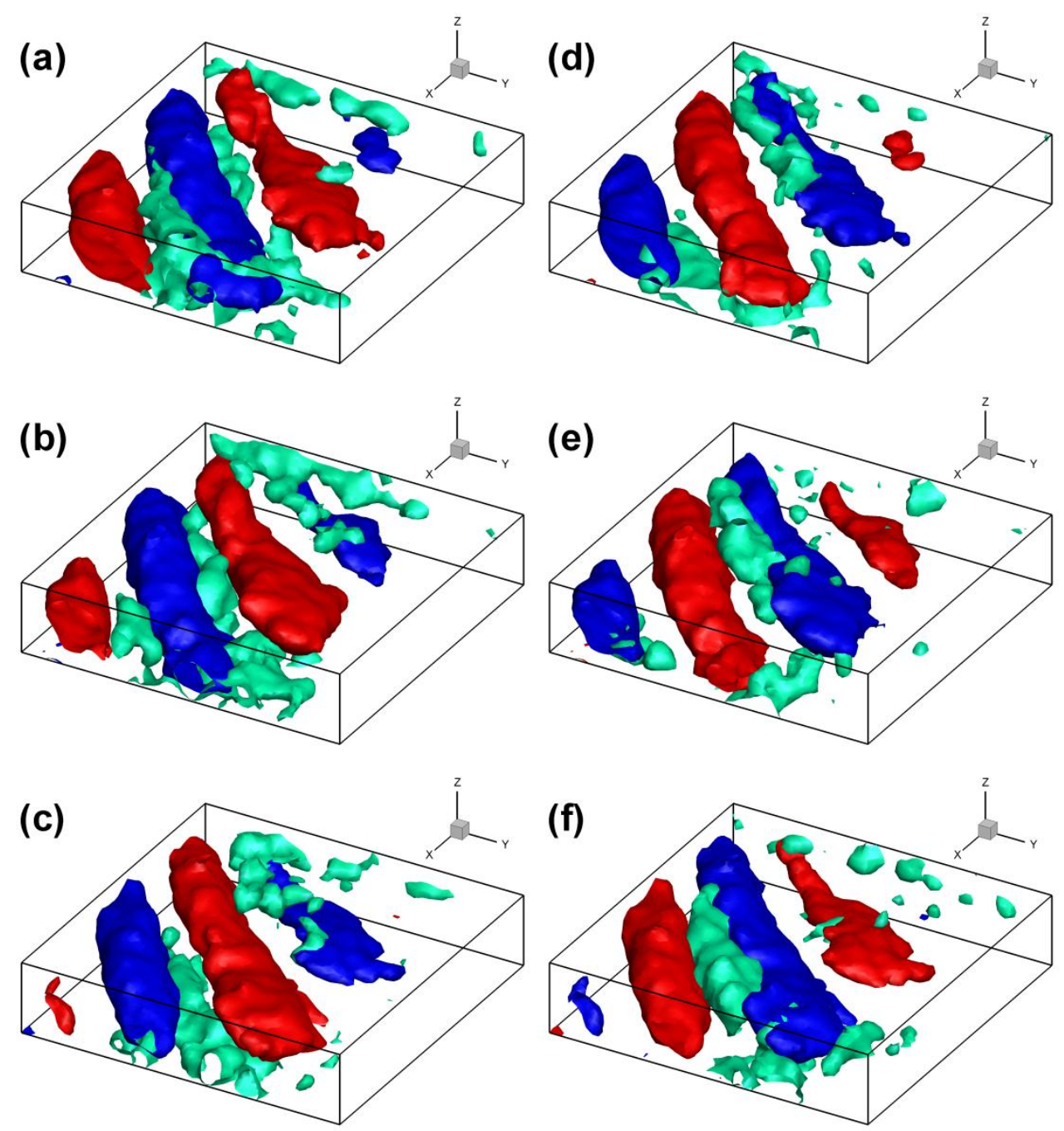

Figure 6- 18 Phase-averaged Reynolds shear stress $-\left\langle u^{\prime} w^{\prime}\right\rangle / U_{0}^{2}=0.014$ (cyan) and POD reconstructed vortices by " $\mathrm{POD}_{1}+\mathrm{POD}_{2}+\mathrm{POD}_{3}+\mathrm{POD}_{4}$ " of the controlled case. The phases are obtained between the $\mathrm{POD}_{1}$ and $\mathrm{POD}_{2}$. Positive (red) and negative (blue) iso-surfaces indicate normalized streamwise vorticity $\omega_{\mathrm{x}}{ }^{*}=\omega_{\mathrm{x}} \cdot \mathrm{h} / \mathrm{U}_{0}= \pm 0.03$. (a)-(f) $\varphi_{1,2}=0^{\circ}, 60^{\circ}, 120^{\circ}, 180^{\circ}, 240^{\circ}, 300^{\circ}$

\subsubsection{DMD analysis based on POD reconstruction}

Being different from the previous DMD applications in the transitional boundary layer at $\mathrm{Re}=$ 800 (Tang et al. 2012) and in a transitional jet at $\mathrm{Re}=5,000$ (Schmid et al. 2012), the present turbulent shear flow $\left(\operatorname{Re}_{\mathrm{h}}=2.0 \times 10^{4}\right)$ is governed by strong turbulent mixing and contains a large range of scales of vortices. In order to analyze dynamic features of the coherent structures, the DMD method is applied to the POD reconstructed flow field which contains the first 14 POD 
modes and the mean flow field:

$$
\widehat{\mathbf{U}_{\mathbf{n}}}=\overline{\mathbf{U}}+\sum_{\mathrm{i}=1}^{\mathrm{N}=14} \mathrm{a}_{\mathrm{i}} \cdot \phi_{\mathrm{i}}
$$

Instantaneous velocity vector fields of an original flow field and a POD reconstruction are compared in Figure 6- 19. It is shown Figure 6- 19(b) that the large-scale flow structures, including the accelerated velocity region and the velocity profiles within the shear layer, are well recovered in the POD reconstruction without the small-scale and random fluctuations. In other words, the POD decomposition and reconstruction act as a spatial filter by keeping in the energetic motions and filtering out the turbulent motions and noise. Therefore, the predominant flow structures which are closely related to the VGs are well represented in Figure 6- 19(b), so it is unnecessary to add more POD modes in the reconstruction for an investigation of these specific modes.
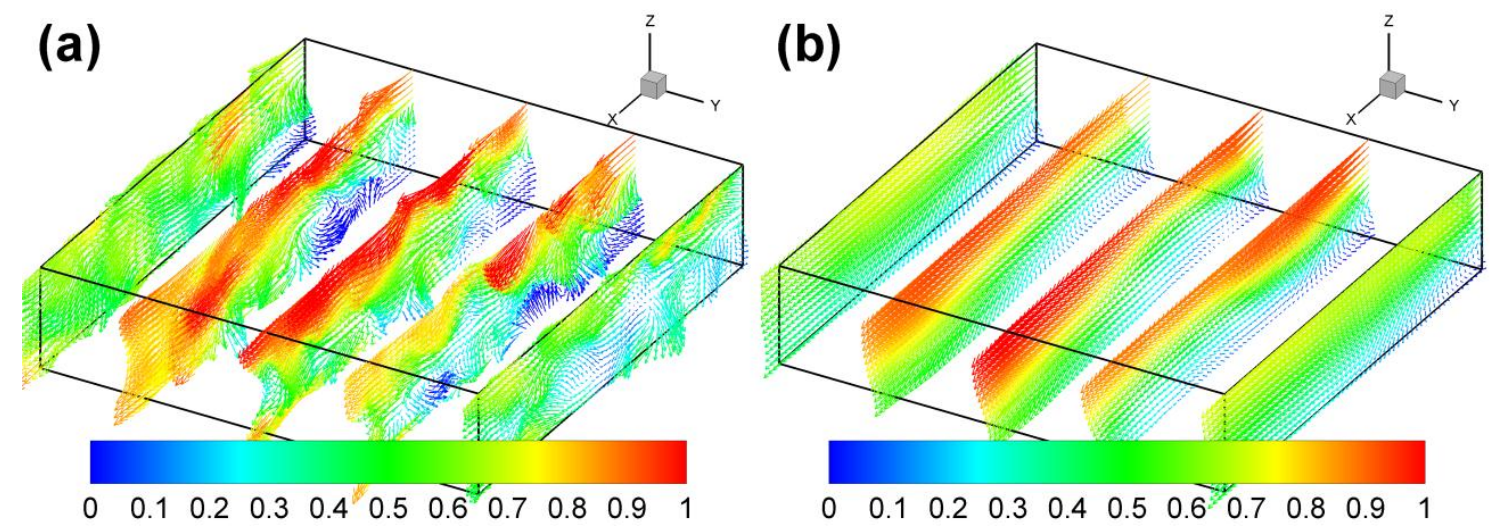

Figure 6- 19 Instantaneous velocity vector fields. (a) original flow field; (b) POD reconstructed flow field.

The vectors and iso-surfaces are color-coded by streamwise velocity component $\overline{\mathrm{u}} / \mathrm{U}_{0}$.

In the next step, the DMD analysis has been applied. The POD reconstructed data is a rank-deficient matrix, so only the first $\mathrm{k}=15$ singular values are valid in the sense of physical meaning. So the diagonal matrix $\boldsymbol{\Sigma}_{\text {svd }}$ can be cut off to:

$$
\boldsymbol{\Sigma}_{\text {svd }}^{\prime}=\left[\begin{array}{ccc}
\mathrm{s}_{1} & & \\
& \ddots & \\
& & \mathrm{s}_{\mathrm{k}}
\end{array}\right]_{\mathrm{k} \times \mathrm{k}}
$$

Then the resulting reduced-sized matrix $\mathbf{S}^{\prime}$ is a $15 \times 15$ full rank matrix. Figure 6- 20 shows the distribution of the absolute values of the DMD amplitudes in the spectrum, which measures the 
importance of each DMD mode: the mode $\mathrm{DMD}_{1}$ has a complex frequency $\mathrm{f}_{1}=(-543.0,0) \mathrm{s}^{-1}$ which corresponds to the time-averaged flow field at $\mathrm{St}_{\mathrm{h}}=0$. It contributes to the highest amplitude of the flow; a predominant mode $\mathrm{DMD}_{8}$ has a complex frequency $\mathrm{f}_{8}=(-1096.0,123.1)$ $\mathrm{s}^{-1}$ at $\mathrm{St}_{\mathrm{h}}=0.37$ and has a high amplitude as well in the spectrum, as marked by a red ellipse. The negative real part of the complex frequency indicates a decaying process in the turbulent shear layer. It can be seen that $\mathrm{DMD}_{8}$ and three neighboring modes form a peak in the spectrum, each of which has different contributions to the coherent structures. This four-DMD-mode peak locates in the "coherent bandwidth" of $0.3<\mathrm{St}_{\mathrm{h}}<0.6$ of the POD analysis in Figure 6- 14.



Figure 6- 20 DMD amplitude distribution in the frequency domain. The negative frequencies are omitted.

The DMD reconstruction is performed by the multiplication of the mode $\mathrm{DMD}_{8}$, the amplitude $\left|a m p_{8}\right|$ and the time evolution $\mathrm{e}^{2 \pi \cdot \mathrm{f}_{8} \cdot \Delta \mathrm{t}}$, which leads to moving vortex structures in the measurement volume (Figure 6- 21). The color bar is omitted because the magnitudes of flow quantities decrease significantly due to the large temporal decay. Quasi-periodic counter-rotating vortices organize in a $\Lambda$-shape and evolve at the single-frequency. Moreover, the vortex structures " $A+C$ " and " $B+D$ " in Figure 6- 21(a) are equivalent to spatial combinations of the POD reconstructed series of vortices "A", "B", "C" and "D" (as shown in Figure 6- 16 and Figure 6- 17) and are self-organized as asymmetric $\Lambda$-shapes. 

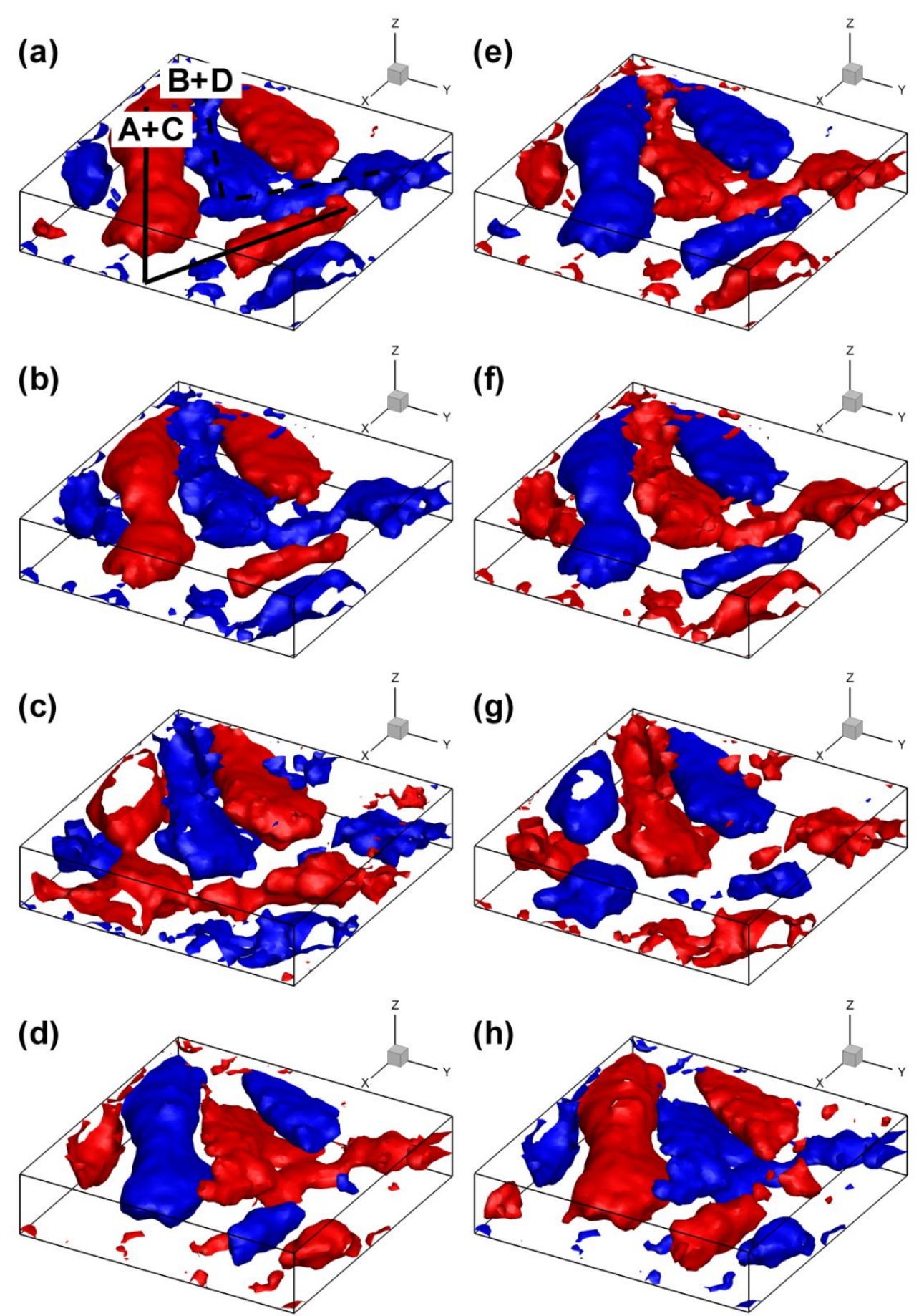

Figure 6- 21 Temporal evolutions of reconstruction by $\mathrm{DMD}_{8}$. (a)-(h) $t=1,2, \ldots, 8$ ms. Positive (red) and negative (blue) iso-surfaces of normalized streamwise vorticity have varying values due to the temporal decay.

The asymmetric formation may be either due to the mean spanwise flows or the difference of turbulent kinetic energy content. This phenomenon of the asymmetry was also observed for 
VGs in turbulent boundary layers by Pauley et al. (1988). The formation of these $\Lambda$-shaped vortex structures can be described in a sequence of three main steps:

(1) the counter-rotating streamwise vortices are generated by the VGs;

(2) the vortices induce the spanwise flows and result in the accelerated velocity region downstream of the VGs;

(3) the streamwise vortices are self-organized from parallel configuration to $\Lambda$-shapes and are elongated in the streamwise direction. These $\Lambda$-shaped vortices play an important role in increasing the Reynolds stress and turbulent kinetic energy in the shear layer (Ma et al. 2014b). Due to the strong turbulent mixing at the high Reynolds number, the $\Lambda$-shaped vortices are hardly visualized directly from the instantaneous snapshot or time-averaged flow field, but the vortex structures and corresponding coherent features can be revealed by POD and DMD in a complementary way.

Therefore, some remarks of the vortex generators are discussed. First, as passive flow control devices, low-profile VGs result in an apparent time-averaged reduction of reattachment length by $29.1 \%$. Second, the VG-induced streamwise counter-rotating vortices form a $\Lambda$-shape vorticity profile and apparently increase the Reynolds shear stress downstream of the VGs. Third, the quasi-periodic oblique vortices are extracted by POD and the "coherent bandwidth" is found, in which the vortex structures show high coherent features. The counter-rotating streamwise vortices are generated by the interaction of the VG-induced vortices and the turbulent shear layer downstream of the BFS which are self-organized as $\Lambda$-shapes.

"Each turbulent flow is different."

Herbert Oertel (1944- ) 


\section{Conclusions and outlook}

This dissertation presents the experimental investigation of coherent structures generated by active and passive separation control in turbulent backward-facing step flow. Three types of flow control devices, including the acoustic tube, oscillating flap and vortex generators, have been applied in the turbulent BFS flow in order to control the flow separation downstream of the step. The experimental investigations have been carried out in the 1-m low-speed wind tunnel at the German Aerospace Center (DLR) in Göttingen, Germany. High-resolution 2D-2C PIV and time-resolved tomographic PIV were used to measure the turbulent boundary layer, separated shear layer and the reattachment area. The spatial structures and dynamic features of the device-induced coherent structures are analyzed by means of multiple post-processing methods, such as the phase-averaging, spatial and temporal cross-correlations, velocity triple decomposition, proper orthogonal decomposition and dynamic mode decomposition. The flow control effects are shown in the time-averaged flow fields that the AT, OF and VGs are able to reduce the reattachment length by $43.7 \%, 31.0 \%$ and $29.1 \%$, respectively. It is shown that large-scale quasi-periodic vortex structures, which are generated by the flow control devices, lead to considerable increases of Reynolds shear stress within the shear layer and result in apparent reductions of the reattachment length. As the coherent structures play an important role in the momentum transfer of the turbulent shear layer flows, the practicability of active and passive devices which artificially induce coherent structures for flow control purposes is once 
again demonstrated to be a viable method in scientific research and engineering flow applications. The essential results, analyses and discussions are summarized as follows.

\subsection{Conclusions}

Acoustic tube: The acoustic tube is an active flow control device, which generates acoustic perturbations in the streamwise direction near the separation edge. The periodic perturbations at $\mathrm{St}_{\mathrm{h}}=0.3\left(\mathrm{f}_{\mathrm{a}}=100 \mathrm{~Hz}\right)$ is close to the fundamental frequency of the separated shear layer, and therefore generate quasi-periodic spanwise vortices shedding downstream. A comparison between the time-averaged flow fields of the clean and controlled cases shows that the acoustic tube is able to reduce the reattachment length by $43.7 \%$. The phase-averaged flow fields provide insight into the processes of vortex rolling-up and breaking down, which result in an increase of the Reynolds shear stress. It is revealed that the artificially enhanced entrainment is more effective in the momentum transfer than the turbulent mixing due to the natural turbulent shear layer development. The coherent structures are extracted by the snapshot POD method and primary and secondary series of vortices are represented by the pairs of modes: $\mathrm{POD}_{1}+\mathrm{i} \cdot \mathrm{POD}_{2}$ and $\mathrm{POD}_{5}+\mathrm{i} \cdot \mathrm{POD}_{6}$. The coherent feature between two POD modes is represented by the circle distributions of the two coefficients. So the relative standard deviation (RSD) is used in order to quantitatively describe the radial distribution of the coefficient points. The primary and the secondary series of vortices correspond to the fundamental frequency and the harmonic of the acoustic tube.

Oscillating flap: The oscillating flap is an active flow control device, which is driven by vertical periodic Ampere force and generates small perturbations at the backward-facing step. The periodic small perturbations have been generated over the separated edge at a subharmonic frequency of $\mathrm{St}_{\mathrm{h}}=0.165\left(\mathrm{f}_{\mathrm{p}}=55 \mathrm{~Hz}\right)$ compared to the most-amplified frequency of the shear layer. The 2D-2C PIV measurements show that the large-scale separated shear layer follows the perturbations upwards and downwards in a flapping motion. Quasi-periodic spanwise vortices are generated downstream, resulting in a time-averaged reduction of the reattachment length by 
$31.0 \%$ downstream of the BFS. Phase-averaged flow fields reveal the spatial evolution and entrainment process of the coherent structures. As the oscillating flap moves downward to the neutral position, it pushes the boundary layer and generates a small streamwise ejection with a velocity of $0.7 \cdot \mathrm{U}_{0}$ into the shear layer. At the opposite phase position, as the oscillating flap moves upwards to the neutral position, it pulls the separated shear layer upwards as well. The flapping motion of the initial part of the shear layer exhibits an identical frequency as the periodic small perturbations and generates two-dimensional waves. In the POD analysis, the coherent structures are extracted by the two predominant POD modes and are reconstructed as quasi-periodic spanwise vortices which move downstream. Furthermore, by applying the spatial DMD method to the POD reconstructed data, a single-wavenumber wave with a decreasing amplitude is identified in the shear layer. It is shown that the periodic small perturbations are able to effectively manipulate the large-scale separated shear layer and generated quasi-periodic coherent structures downstream.

Vortex generators: The low-profile wedge-type vortex generators have been applied to the backward-facing step flow. As the VG heights are lower than the thickness of the turbulent boundary layer, they are submerged within the boundary layer. The instantaneous velocity vector field indicates a highly turbulent state and shows that more vortex structures are generated by the VGs. Among the four configurations, the case "VG-H10b" ( $\mathrm{H}=10 \mathrm{~mm}$, backward-oriented) results in an apparent time-averaged reduction of reattachment length by $29.1 \%$, which is a better value than for the other VG configurations. The VG-induced streamwise counter-rotating vortices form a $\Lambda$-shape vorticity profile and apparently increase the Reynolds shear stress downstream of the VGs. The spatial and temporal cross-correlations reveal the streamwise periodic structures moving at the convection velocity of $\mathrm{U}_{\mathrm{c}} \approx 0.38 \cdot \mathrm{U}_{0}$. POD and DMD have been applied to the time-resolved tomographic PIV data in a complementary way in order to analyze the spatial structures and dynamic features of the VG-induced vortices in the turbulent shear layer downstream of the BFS. By the snapshot POD method, the quasi-periodic oblique vortices are extracted and the "coherent bandwidth" is found, 
in which the vortex structures show high coherent features. By applying the spatial DMD method to the POD reconstructed data, a predominant dynamic mode is found which represents the $\Lambda$-shaped vortex structures evolving as a single-frequency wave in the turbulent shear layer. It is shown that the counter-rotating streamwise vortices are generated by the interaction of the VG-induced vortices and the turbulent shear layer downstream of the BFS which are self-organized as $\Lambda$-shapes.

\subsection{Outlook}

A few disadvantages of the designs of the flow control devices should be clarified. Limitations of the PIV measurements and post-processing should also be discussed. This self-critical discussion is not easy, but it essentially benefits future work.

The acoustic tube has an asymmetric design because the inner sound wave is generated by the loudspeaker on one side of the tube and reflected on the other side. Apparently, the sound pressure level along the open slot $(150 \mathrm{~mm})$ is not constant: the side closer the loudspeaker is a few $\mathrm{dB}$ higher than the other side. In future, two identical loudspeakers can be employed on both side of the acoustic tube in order to generate an uniform acoustic perturbation amplitude at the open slot. Moreover, the frequencies are fixed at $\mathrm{f}_{\mathrm{a}}=100,300,500,700$ and $900 \mathrm{~Hz}$ due to the principle of standing wave. An adjustable length of the tube could be considered for producing flexible frequencies.

The oscillating flap provides only one perturbation frequency of $f_{p}=55 \mathrm{~Hz}$ due to the fixed length, linear density and tension force within the flap. In future design, the tension force can be adjustable in order to produce the fundamental frequency and the harmonics.

The VG have various parameters, such as height, orientation, length, spacing, shape and so on. In the present work, only the first two parameters have been studied due to limited time and tight schedule. Future research can focus on the influence of other parameter sets of various 
types of VGs.

Although the 2D-2C PIV can achieve a high spatial resolution for velocity vector fields, time information is missing. In future research, time-resolved measurements may be complemented to analyze frequency spectra of the turbulent shear layer and temporal features of the coherent structures.

There are still more questions than answers on the coherent structures in turbulent shear flows. Based on linear analyses, the POD and DMD methods have their limitations in interpreting nonlinear processes in turbulent flows. In future, nonlinear research, consisting of the detection and analysis of spatial and temporal evolving events of Reynolds stresses, may help to improve the understanding of the vortex topology and development in the turbulent shear flow.

"When I meet God, I am going to ask him two questions: Why relativity? And why turbulence? I really believe he will have an answer for the first."

Werner K. Heisenberg (1901-1976) 


\section{Appendix A. Photographs of experiments}

Figure A- 1 Calibration of the acoustic tube. The input is the sine wave signals into the loudspeaker and the output is the sound pressure level at the exit of the slot.

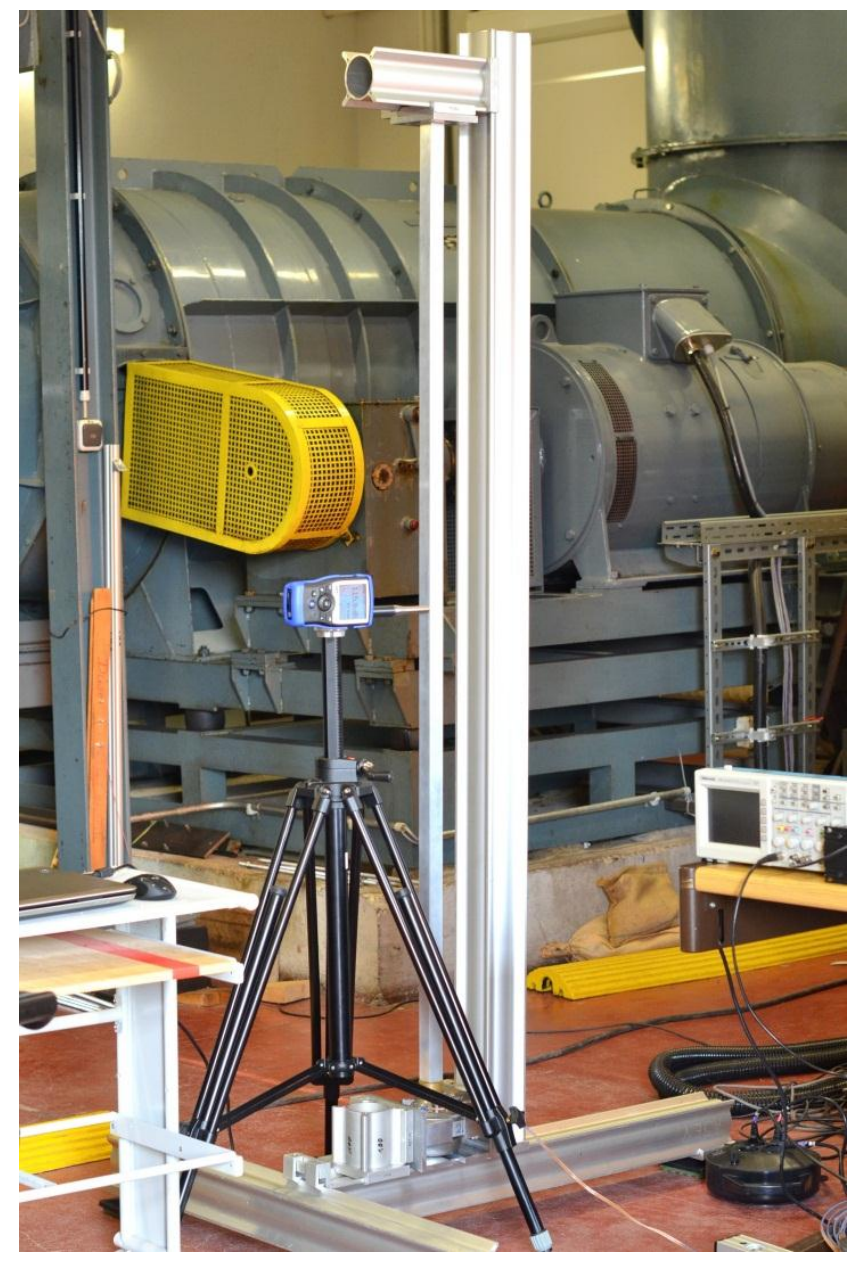


Figure A- 2 Preliminary test of the oscillating flap before wind tunnel test

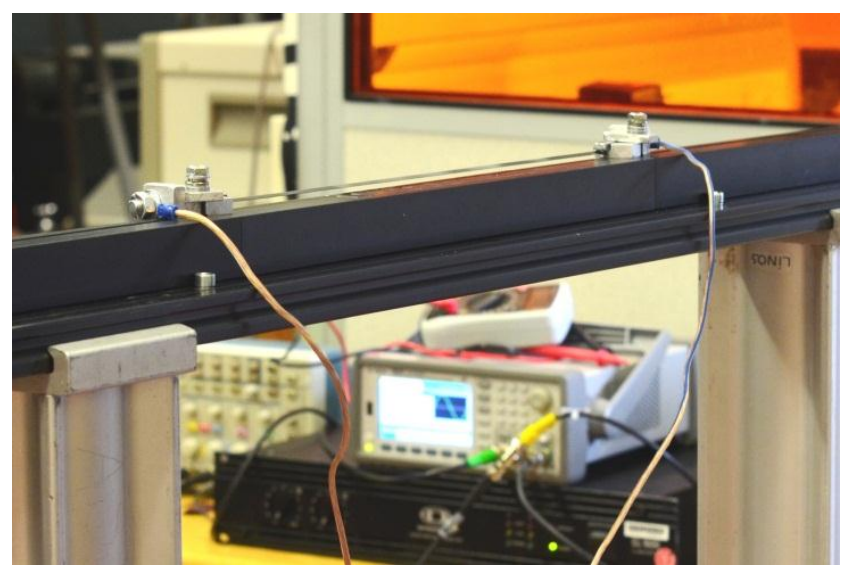

Figure A- 3 Acoustic measurement of the sound pressure level near the center part of the oscillating flap

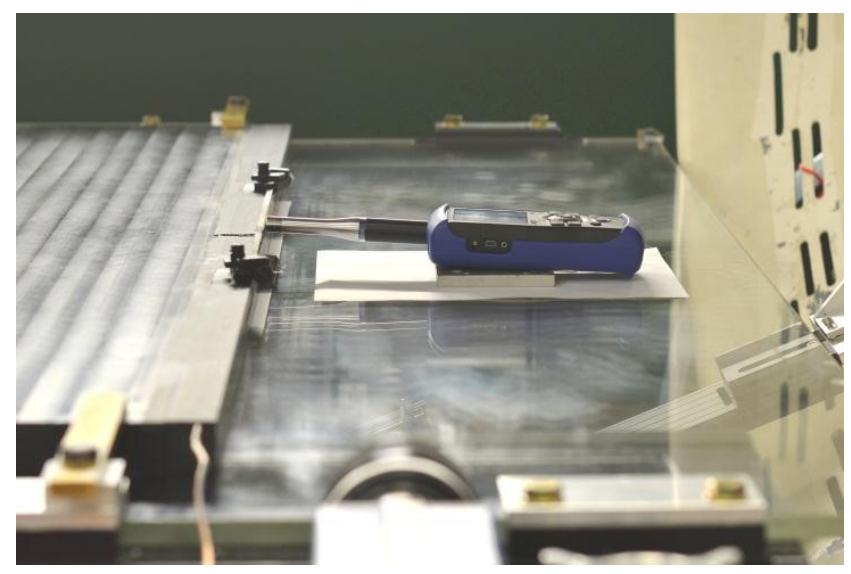

Figure A- 4 Signal generation system consisting of a waveform generator, power amplifier and oscilloscope

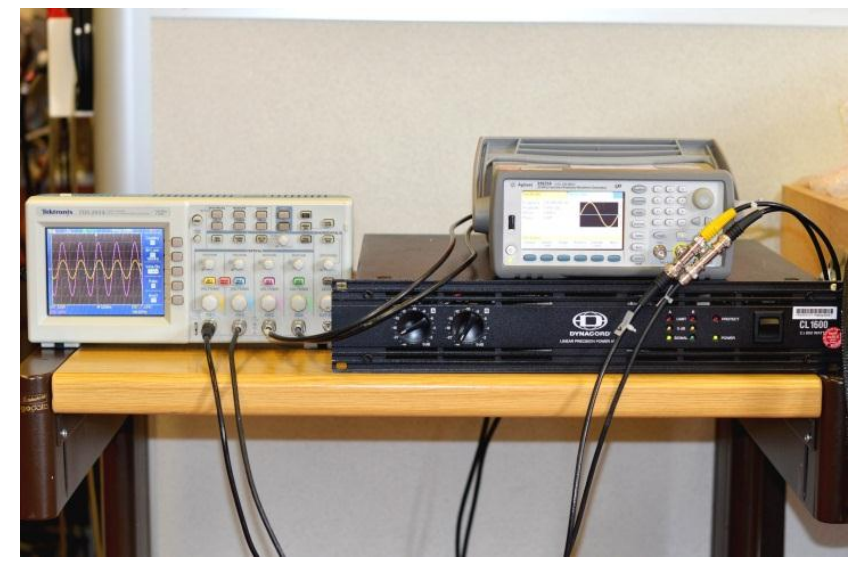


Figure A- 5 Vortex generators $(H=10$

$\mathrm{mm}$, forward-orientated) on the backward-facing step



Figure A- 6 Vortex generators $(\mathrm{H}=5$ $\mathrm{mm}$, forward-orientated) on the backward-facing step

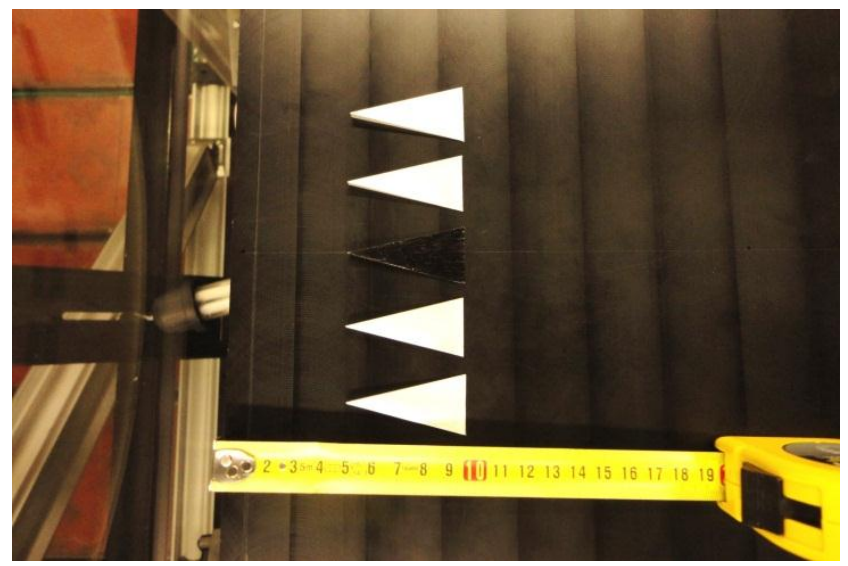

Figure A- 7 Zigzag bands near the leading edge of the flat plate




Figure A- 8 2D-2C PIV calibration



Figure A- 9 PCO.4000 high-resolution camera with the Nikon lens $(85 \mathrm{~mm}, \mathrm{f} / 4)$

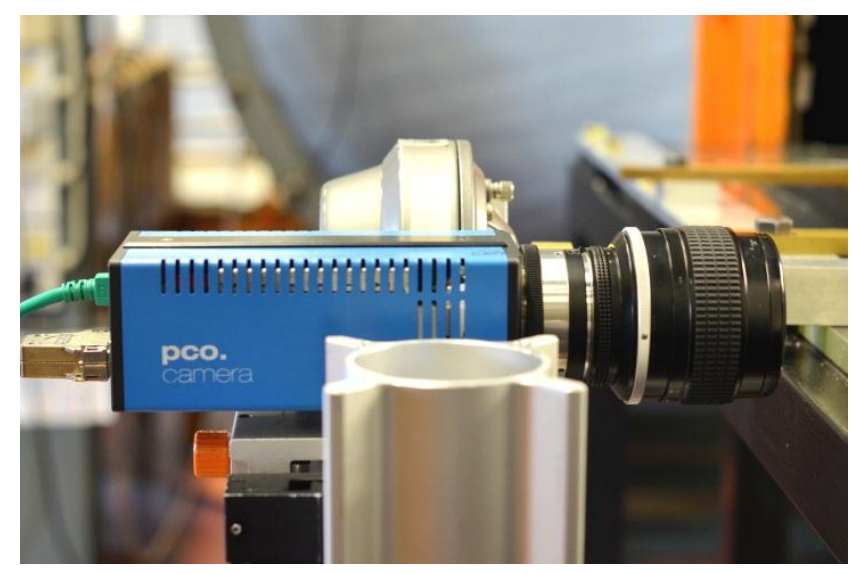

Figure A-10 Front side of the Nikon lens (85mm, f/4)

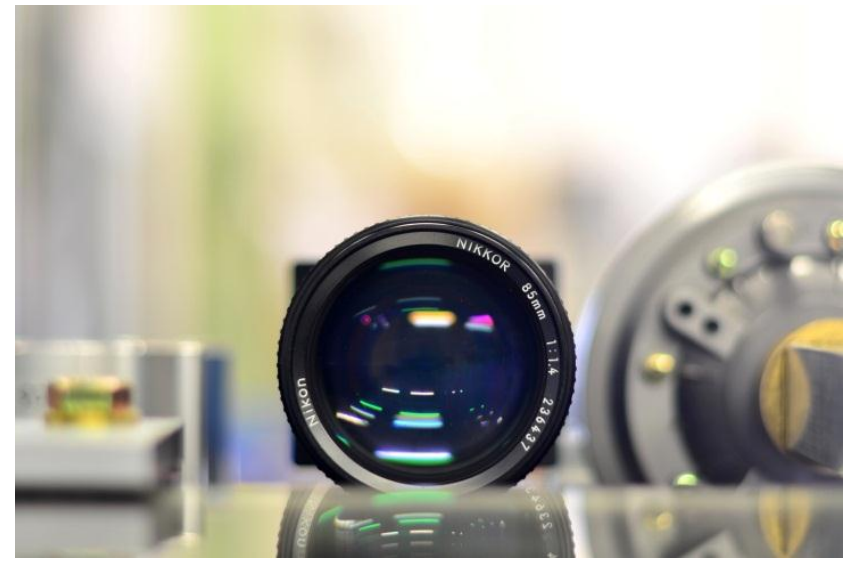


Figure A- 11 Ultra CFR Nd:YAG laser system from Big Sky inc.

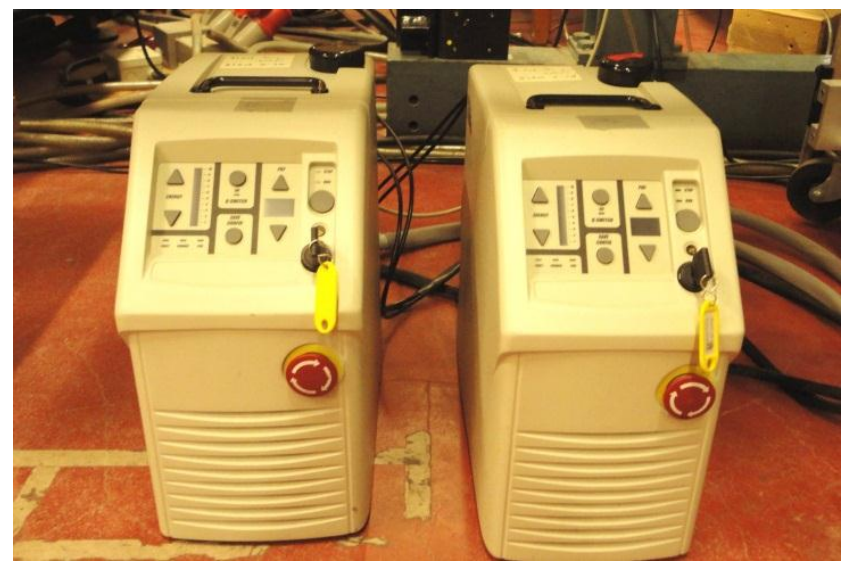

Figure A- 12 Plane mirror mounted downstream of the BFS

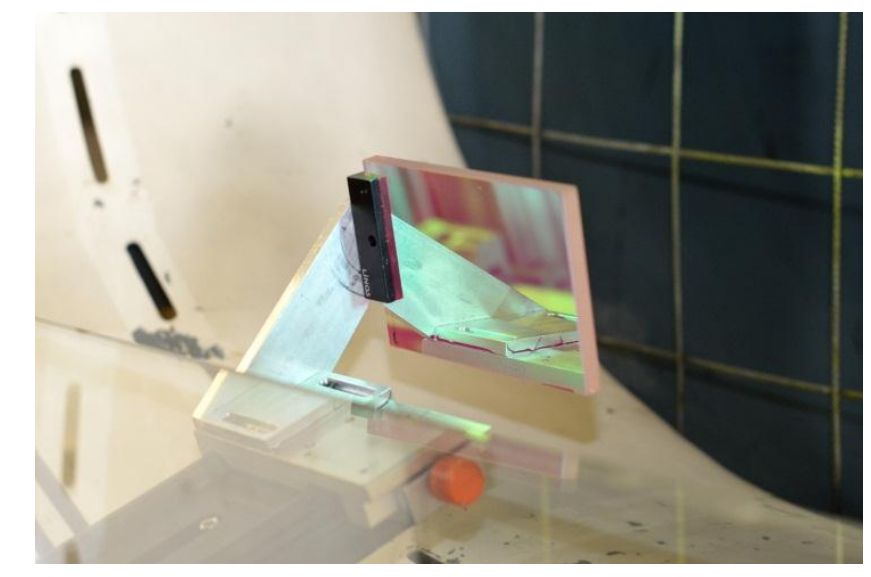

Figure A- 13 Laser light sheet in the 2D-2C PIV measurement of the acoustic tube

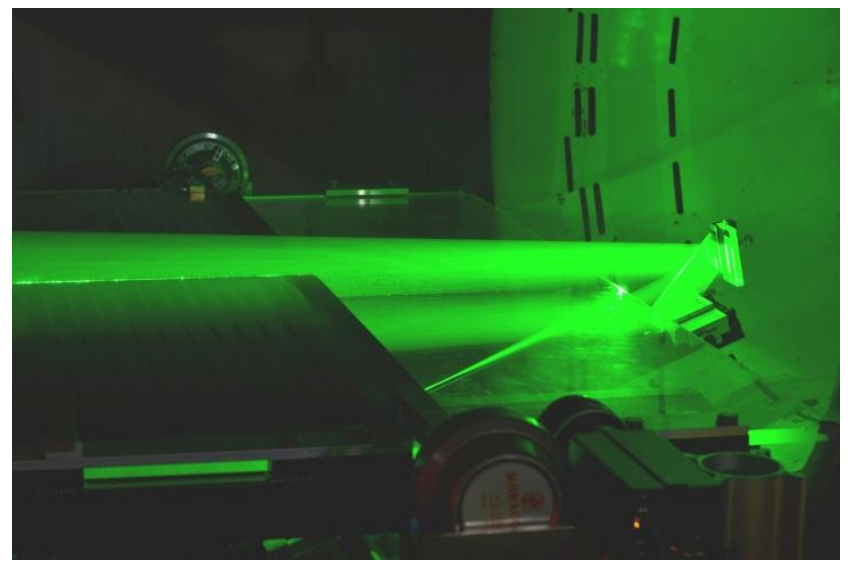


Figure A- 14 Laser light sheet in the 2D-2C PIV measurement of the oscillating flap

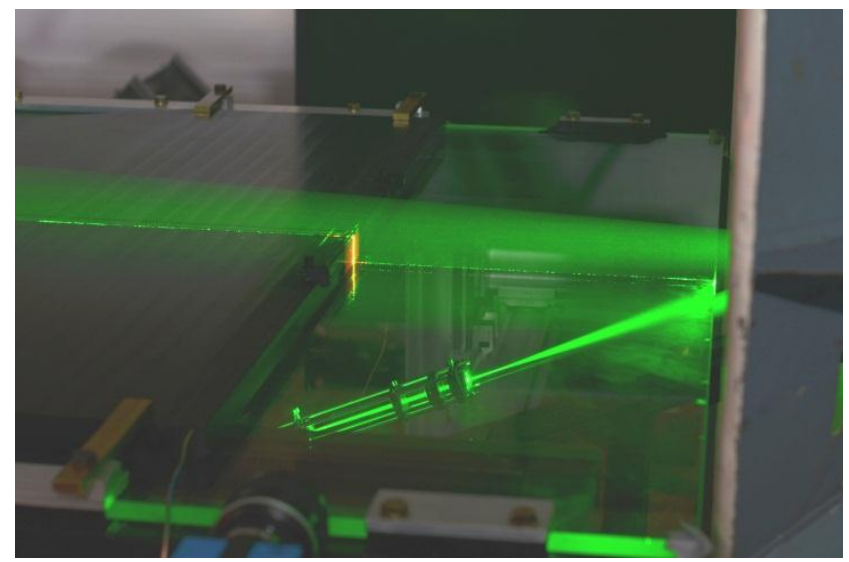

Figure A- 15 Tomographic PIV calibration

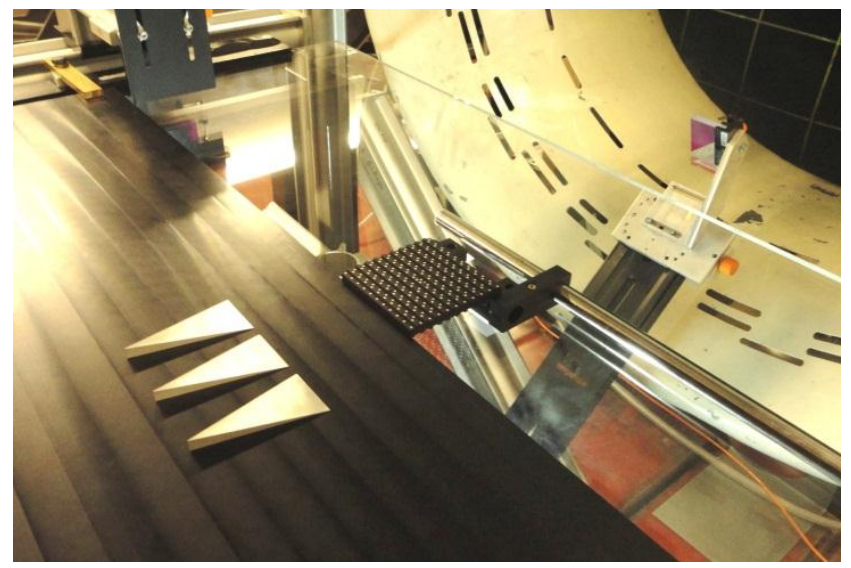

Figure A- 16 Four Photron APX-RS high-speed cameras with Nikon lenses (105mm, f/8)




Figure A- 17 High-repetition-rate laser generator from LEE inc.

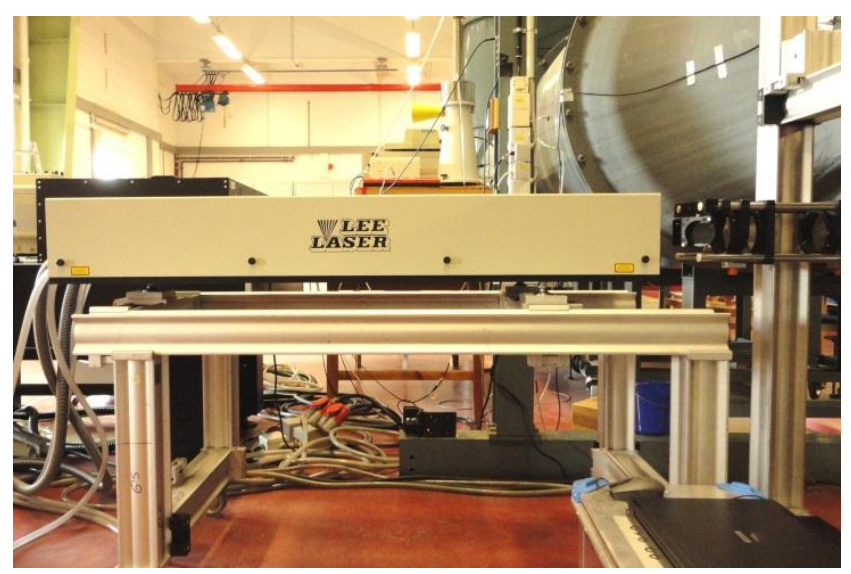

"Turbulence may indeed continue to remain nature's best-kept secret, but we should persist in our efforts to unveil these secrets."

A. K. M. Fazle Hussain (1943- ) 


\section{Appendix B. Publications}

\section{Publications}

Ma X, Geisler R, Agocs J, Schröder A (2015) Investigation of coherent structures generated by acoustic tube in turbulent flow separation control. Experiments in Fluids 56:46

Ma X, Geisler R, Agocs J, Schröder A (2014) Investigation of coherent structures in active flow control over a backwar-facing step by PIV. In: Proceeding of 16th International Symposium on Flow Visualization, Okinawa, Japan

Ma X, Geisler R, Agocs J, Schröder A (2014) Time-resolved tomographic PIV investigation of turbulent flow control by vortex generators on a backward-facing step. In: Proceeding of 17th International Symposium on Applications of Laser Techniques to Fluid Mechanics, Lisbon, Portugal

\section{Conferences and workshops}

Annual workshop on Measurement and Optical Techniques for Aerospace Research at the German Aerospace Center (DLR), Cologne, Germany, 2015

17th International Symposium on Applications of Laser Techniques to Fluid Mechanics, Lisbon, Portugal, 2014

16th International Symposium on Flow Visualization, Okinawa, Japan, 2014

16th STAB-workshop at the German Aerospace Center (DLR), Göttingen, Germany, 2013

Lecture Series on Advanced post-processing of experimental and numerical data at the Von Karman Institute for Fluid Dynamics, Brussels, Belgium, 2013

20th PIV Course at the German Aerospace Center (DLR), Göttingen, Germany, 2012

3rd PSP Course at the German Aerospace Center (DLR), Göttingen, Germany, 2011

German language course at the Goethe-Institute Göttingen, Germany, 2011 


\section{Lectures and teaching assistance in the University of Göttingen}

Lectures on "Stellar structure and evolution", 2013

Seminar on "Self-organization in Physics and Biology" at the Max Planck Institute for Dynamics and Self-organization, 2013

Teaching assistance on "Computergestütztes Wissenschaftliches Rechnen 2 (Scientific calculation 2)", 2013

Seminar on "Papers, proposals, presentations-skills of scientific communication", 2013

Seminar on "Current Questions in Turbulent Research" at the Max Planck Institute for Dynamics and Self-organization, 2012

Teaching assistance on "Physik 1 (Physics 1)", 2012

Lectures on "Data analysis in astrophysics", 2012

German language course, 2012

"The importance of asking the right question is as obvious in principle as it is nebulous in research. Always present is the temptation to look for the key under the street light and not in the dark corner where it was lost."

Hans W. Liepmann (1914-2009) 


\section{References}

[1] Ackeret J (1925) Das Rotorschiff und seine physikalischen Grundlagen. Vandenhoeck und Rupprecht, Göttingen

[2] Adrian RJ (2005) Twenty years of particle image velocimetry. Exp Fluids 39:159-169

[3] Adrian RJ, Meinhard CD, Tomkins CD (2000) Vortex organization in the outer region of the turbulent boundary layer. J Fluid Mech 422:1-54

[4] Armaly BF, Durst F, Pereira JCF, Schönung B (1983) Experimental and theoretical investigation of backward-facing step flow. J Fluid Mech 127:473-496

[5] Ashill PR, Fulker JL, Hackett KC (2001) Research at DERA on sub boundary layer vortex generators (SBVGs). AIAA 2001-0887, In: Proceedings of the 39th AIAA Aerospace Sciences Meeting and Exhibit, Reno, USA

[6] Ashill PR, Fulker JL, Hackett KC (2002) Studies of flows induced by sub boundary layer vortex generators (SBVGs). AIAA paper 2002-0968, In: Proceedings of the 40th AIAA Aerospace Sciences Meeting and Exhibit, Reno, USA

[7] Bade KM, Foss JF (2010) Attributes of the large-scale coherent motions in a shear layer. Exp Fluids 49:225-239

[8] Berkooz G, Holmes P, Lumley JL (1993) The proper orthogonal decomposition in the analysis of turbulent flows. Annu Rev Fluid Mech 25:539-575

[9] Bernero S, Fiedler HE (2000) Application of particle image velocimetry and proper orthogonal decomposition to the study of a jet in a counterflow. Exp Fluids 29:S274-S281

[10] Betterton JG, Hackett KC, Ashill PR, Wilson MJ, Woodcock IJ, Tilman CP, Langan KJ (2000) Laser Doppler Anemometry investigation on sub boundary layer vortex generators for flow control. In: Proceedings of 10th International Symposium on Applications of Laser 
Techniques to Fluid Mechanics, Lisbon, Portugal

[11] Bhattacharjee S, Scheelke B, Troutt TR (1986) Modification of vortex interaction in a reattaching separated flow. AIAA J 24(4):623-629

[12] Bian S, Driscoll JF, Elbing BR, Ceccio SL (2011) Time resolved flow-field measurements of a turbulent mixing layer over a rectangular cavity. Exp Fluids 51:51-63

[13] Blackwelder RF, Kovasznay SG (1972) Time scales and correlations in a turbulent boundary layer. Phys Fluids 15:1545

[14] Bonnet JP, Cole DR, Delville J, Glauser MN, Ukeiley LS (1994) Stochastic estimation and proper orthogonal decomposition: complementary techniques for identifying structure. Exp Fluids 17:307-314

[15] Brown GL, Roshko A (1974) On density effects and large structure in turbulent mixing layers. J Fluid Mech 64(4):775-816

[16] Brun C, Aubrum S, Goossens T, Ravier Ph (2008) Coherent structures and their frequency signature in the separated shear layer on the sides of a square cylinder. Flow Turbul Combust 81:97-117

[17] Bushnell DM (1991) Drag reduction in nature. Annu Rev Fluid Mech 23:65-79

[18] Bushnell DM, McGinley CB (1989) Turbulence control in wall flows. Annu Rev Fluid Mech 21:1-20

[19] Cantwell BJ (1981) Organized motion in turbulent flow. Annu Rev Fluid Mech 13:457-515

[20] Cattafesta LN, Sheplak M (2011) Actuators for active flow control. Annu Rev Fluid Mech $43: 247-272$

[21] Chandrsuda C, Mehta RD, Weir AD, Bradshaw P (1978) Effect of free-stream turbulence on large structure in turbulent mixing layers. J Fluid Mech 85(4):693-704

[22] Chao YC, Han JM, Jeng MS (1990) A quantitative laser sheet image processing method for the study of the coherent structure of a circular jet flow. Exp Fluids 9:323-332

[23] Choi H, Jeon WP, Kim J (2008) Control of flow over a bluff body. Annu Rev Fluid Mech 40:113-139

[24] Chun KB, Sung HJ (1996) Control of turbulent separated flow over a backward-facing step 
by local forcing. Exp Fluids 21:417-426

[25] Clauser FH (1956) The turbulent boundary layer. Adv Appl Mech 4:1-51

[26] Corino ER, Brodkey RS (1969) A visual investigation of the wall region in turbulent flow. J Fluid Mech 37(1):1-30

[27] Crow SC, Champagne FH (1971) Orderly structure in jet turbulence. J Fluid Mech 48(3):547-591

[28] de Brederode V and Bradshaw P (1978) Influence of the side walls on the turbulent center-plane boundary layer in a square duct. J Fluids Eng 100:91-96

[29] Doetsch H (1940) Untersuchungen an einigen Profilen mit geringem Widerstand im Bereich kleiner $\mathrm{c}_{\mathrm{a}}$-Werte. Luftfahrtforschung 1:54-57

[30] Eaton JK, Johnston JP (1981) A review of research on subsonic turbulent-flow reattachment. AIAA J 19:1093-1100

[31] Elsinga GE, Scarano F, Wieneke B, van Oudheusden BW (2006) Tomographic particle image velocimetry. Exp Fluids 41:933-947

[32] Falco RE (1977) Coherent motions in the outer region of turbulent boundary layers. Phys Fluids 20:S124

[33] Faulders CR (1961) A note on laminar boundary-layer skin friction under the influence of foreign-gas injection. J Aerospace Science 28(2):166-167

[34] Favre AJ, Gaviglio JJ, Dumas R (1957) Space-time double correlations and spectra in a turbulent boundary layer. J Fluid Mech 2(4):313-342

[35] Flatt J (1961) The history of boundary layer control research in the United States of America. In: Lachmann GV ed. Boundary Layer and Flow Control, Pergamon Press UK $1: 122-143$

[36] Försching H, Hennings H (2012) Aeroelastic mysteries in avian flight. CEAS Aeronaut J $3: 135-143$

[37] Freymuth P (1966) On transition in a separated laminar boundary layer. J Fluid Mech 25(4):683-704

[38] Gad-el-Hak M, Pollard A, Bonnet JP (1998) Flow Control: Fundamentals and Practices. 
Springer-Verlag Germany

[39] Gao Q, Ortiz-Duenas C, Longmire EK (2013) Evolution of coherent structures in turbulent boundary layers based on moving tomographic PIV. Exp Fluids 54:1625

[40] Goett HJ, Bullivant WK (1939) Tests of N.A.C.A. 0009, 0012, and 0018 airfoils in the full-scale tunnel. NACA Technical Report No. 647

[41] Gross JF, Hartnett JP, Masson DJ, Gazley CJr (1961) A review of binary boundary layer characteristics. J Heat Mass Transfer 3:108-221

[42] Gurka R, Liberzon A, Hetsroni G (2006) POD of vorticity fields: A method for spatial characterization of coherent structures. Int J Heat Fluid Fl 27:416-426

[43] Hasan MAZ (1992) The flow over a backward-facing step under controlled perturbation: laminar separation. J Fluid Mech 238:73-96

[44] Ho CM, Huang LS (1982) Subharmonic and vortex merging in mixing layers. J Fluid Mech 119:443-473

[45] Husain HS, Hussain AKMF (1995) Experiments on subharmonic resonance in a shear layer. J Fluid Mech 304:343-372

[46] Hussain AKMF (1986) Coherent structures and turbulence. J Fluid Mech 173:303-356

[47] Hussain AKMF, Reynolds WC (1970) The mechanics of an organized wave in turbulent shear flow. J Fluid Mech 41(2):241-258

[48] Hussain AKMF, Zaman KBMQ (1981) The 'preferred mode' of the axisymmetric jet. J Fluid Mech 110:39-71

[49] Hussain AKMF, Zaman KBMQ (1985) An experimental study of organized motions in the turbulent mixing layer. J Fluid Mech 159:85-104

[50] Jacobs EN, Sherman A (1937) Airfoil section characteristics as affected by variations of the Reynolds number. NACA Technical Report No. 586

[51] Kähler CJ, Sammler B, Kompenhans J (2002) Generation and control of tracer particle for optical flow investigation in air. Exp Fluids 33:736-742

[52] Kim HT, Kline SJ, Reynolds WC (1971) The production of turbulence near a smooth wall in a turbulent boundary layer. J Fluid Mech 50(1):133-160 
[53] Kit K, Krivonosova O, Zhilenko D, Friedman D (2005) Reconstruction of large coherent structures from SPIV measurements in a forced turbulent mixing layer. Exp Fluids $39: 761-770$

[54] Klebanoff PS (1955) Characteristics of turbulence in a boundary layer with zero pressure gradient. NACA Technical Report No. 1247

[55] Kline SJ, Reynolds WC, Schraub FA, Runstadler PW (1967) The structure of turbulent boundary layers. J Fluid Mech 30(4):741-773

[56] Kuethe AM (1972) Effect of streamwise vortices on wake properties associated with sound generation. J Aircraft 9(10) 715-719

[57] Lachmann GV (1961) Boundary Layer and Flow Control. Pergamon Press UK

[58] Laufer J (1975) New trends in experimental turbulence research. Annu Rev Fluid Mech 7:307-326

[59] LeHew JA, Guala M, McKeon BJ (2013) Time-resolved measurements of coherent structures in the turbulent boundary layer. Exp Fluids 54:1508

[60] Liepmann HW (1997) Boundary layer transition: the early days. Appl Mech Rev 50(2):R1-R4

[61] Liepmann HW, Fila GH (1947) Investigation of effect of surface temperature and single roughness elements on boundary layer transition. NaCA Rep 890

[62] Lin JC (2002) Review of research on low-profile vortex generators to control boundary-layer separation. Prog Aerosp Sci 38:389-420

[63] Linke W (1942) Über den Strömungswiderstand einer behaizten ebenen Platte. Luftfahrtforschung 19:157-160

[64] Lumley JL (1967) The Structure of inhomogeneous turbulent flow. Atmospheric Turbulence and Radio Wave Propagation, Moscow, pp 166-178

[65] Ma X, Geisler R, Agocs J, Schröder A (2014a) Investigation of coherent structures in active flow control over a backwar-facing step by PIV. In: Proceeding of 16th International Symposium on Flow Visualization, Okinawa, Japan

[66] Ma X, Geisler R, Agocs J, Schröder A (2014b) Time-resolved tomographic PIV 
investigation of turbulent flow control by vortex generators on a backward-facing step. In: Proceeding of 17th International Symposium on Applications of Laser Techniques to Fluid Mechanics, Lisbon, Portugal

[67] Ma X, Geisler R, Agocs J, Schröder A (2015) Investigation of coherent structures generated by acoustic tube in turbulent flow separation control. Exp Fluids 56:46

[68] McConachie PJ (1981) The distribution of convection velocities in turbulent pipe flow. J Fluid Mech 103:65-85

[69] Mehta RD, Bradshaw P (1988) Longitudinal vortices imbedded in turbulent boundary layers. Part 2. Vortex pair with 'common flow' upwards. J Fluid Mech 188:529-546

[70] Meyer KE, Pedersen JM, Özcan O (2007) A turbulent jet in crossflow analysed with proper orthogonal decomposition. J Fluid Mech 583:199-227

[71] Moin P, Bewley T (1994) Feedback control of turbulence. Appl Mech Rev 49(6):S3-S13

[72] Pan C, Yu D, Wang JJ (2011) Dynamic mode decomposition of Gurney flap wake flow. Theoretical and Applied Mechanics Letters 1:012002

[73] Pauley WR, Eaton JK (1988) Experimental study of the development of longitudinal vortex pairs embedded in a turbulent boundary layer. AIAA J 26(7):816-823

[74] Pedersen JM, Meyer KE (2002) POD analysis of flow structures in a scale model of ventilated room. Exp Fluids 33:940-949

[75] Perrin R, Braza M, Cid E, Cazin S, Barthet A, Sevrain A, Mockett C, Thiele F (2007) Obtain phase averaged turbulence properties in the near wake of a circular cylinder at high Reynolds number using POD. Exp Fluids 43:341-355

[76] Prandtl L (1904) Über Flüssigkeitsbewegung bei sehr kleiner Reibung. Proc Third Int Math Congr, Heidelberg, Germany, 484-491

[77] Prandtl L (1925) Magnuseffeckt und Windkraftschiff. Naturwissenschaften 13:93-108

[78] Raffel M, Willert CE, Wereley ST, Kompenhans J (2007) Particle Image Velocimetry: A Practical Guide, 2nd edn. Springer, Berlin

[79] Rao DM, Kariya TT (1988) Boundary-layer submerged vortex generators for separation control - an exploratory study. AIAA paper 88-3546-CP, In: Proceedings of 
AIAA/ASME/SIAM/APS 1st National Fluid Dynamics Congress, Cicinnati, USA

[80] Robinson SK (1991) Coherent motions in the turbulent boundary layer. Annu Rev Fluid Mech 23:601-639

[81] Roos FW, Kegelman JT (1986) Control of coherent structures in reattaching laminar and turbulent shear layers. AIAA J 24(12):1956-1963

[82] Santa Cruz A, David L, Pecheux J, Texier A (2005) Characterization by proper-orthogonal-decomposition of the passive controlled wake flow downstream of a half cylinder. Exp Fluids 39:730-742

[83] Schanz D, Gesemann S, Schröder A, Wieneke B, Novara M (2013) Non-uniform optical transfer functions in particle imaging: Calibration and application to Tomographic reconstruction. Measurement Science and Technology 24(2):1-16

[84] Schanz D, Schröder A, Gesemann S (2014) 'Shake The Box' - a 4D PTV algorithm: Accurate and ghostless reconstruction of Lagrangian tracks in densely seeded flows. In: Proceeding of 17th International Symposium on Applications of Laser Techniques to Fluid Mechanics, Lisbon, Portugal

[85] Schlichting H (1979) Boundary-layer control in laminar flow. In: Boundary-Layer Theory, 7th edn. McGRAW-HILL, New York

[86] Schmid PJ (2010) Dynamic mode decomposition of numerical and experimental data. J Fluid Mech 656:5-28

[87] Schmid PJ, Violato D, Scarano F (2012) Decomposition of time-resolved tomographic PIV. Exp Fluids 52:1567-1579

[88] Schrenk O (1935) Versuche mit Absaugflügeln. Luftfahrtforschung 12:10-27

[89] Schröder A, Geisler R, Elsinga GE, Scarano F, Dierksheide U (2008) Investigation of a turbulent spot and a tripped turbulent boundary layer flow using time-resolved tomographic PIV. Exp Fluids 44:305-316

[90] Schröder A, Geisler R, Staack K, Elsinga GE, Scarano F, Wieneke B, Henning A, Poelma C, Westerweel J (2011) Eulerian and Lagrangian views of a turbulent boundary layer flow using time-resolved tomographic PIV. Exp Fluids 50:1071-1091 
[91] Schröder A, Schanz D, Heine B, Dierksheide U (2013) Investigation of transitional flow structures downstream of a backward-facing-step by using 2D-2C- and high resolution 3D-3C- tomo-PIV. New Results in Numer \& Exp Fluid Mech NNFM 121:219-226

[92] Schröder A, Schanz D, Michaelis D, Cierpka C, Scharnowski S, Kähler CJ (2015) Advances of PIV and 4D-PTV "Shake-The-Box" for turbulent flow analysis - the flow over periodic hills. Flow Turbul Combust DOI:10.1007/s10494-015-9616-2

[93] Schubauer GB, Spangenberg WG (1960) Forced mixing in boundary layer. J Fluid Mech $8(1): 10-32$

[94] Sigurdson LW (1995) The structure and control of a turbulent reattaching flow. J Fluid Mech 298:139-165

[95] Sirovich L (1987) Turbulence and the dynamics of coherent structures. Part 1: coherent structure. Quart Appl Math 45(3):561-571

[96] Spillman JJ (1978) The use of wing tip sails to reduce vortex drag. Aeronaut J 82:378-395

[97] Tang ZQ, Jiang N (2012) Dynamic mode decomposition of hairpin vortices generated by a hemisphere protuberance. Science China: Physics, Mechanics and Anstronomy 55(1):118-124

[98] Taylor GI (1935) Statistical theory of turbulence Part 1-4. Proc R Sco Lond A 151:421-478

[99] Taylor GI (1936) Correlation measurements in a turbulent flow through a pipe. Proc R Sco Lond A 157:537-546

[100] Taylor HD (1947) The elimination of diffuser separation by vortex generators. United Aircraft Corporation Report No. R-4012-3

[101] Townsend AA (1970) Entrainment and the structure of turbulent flow. J Fluid Mech 41(1):13-46

[102] Violato D, Scarano F (2011) Three-dimensional evolution of flow structures in transisional circular and chevron jets. Phys Fluids 23:124104

[103] Wallace JM, Eckelmann J, Brodkey RS (1972) The wall region in turbulent shear flow. J Fluid Mech 54:39-48

[104] Welch PD (1967) The Use of Fast Fourier Transform for the Estimation of Power Spectra: 
A Method Based on Time Averaging Over Short, Modified Periodograms. IEEE Transactions on Audio Electroacoustics, AU-15(2), 70-73

[105] Westerweel J, Elsinga GE, Adrian RJ (2013) Particle image velocimetry for complex and turbulent flows. Annu Rev Fluid Mech 45:409-436

[106] Wieneke B (2008) Volume self-calibration for 3D particle image velocimetry. Exp Fluids $45: 549-556$

[107] Willert CE, Gharib M (1991) Digital particle image velocimetry. Exp Fluids 10:181-193

[108] Willmarth WW, Lu SS (1972) Structure of the Reynolds stress near the wall. J Fluid Mech $55: 65-92$

[109] Wills JAB (1964) On convection velocities in turbulent shear flows. J Fluid Mech 20(3):417-432

[110] Winant CD, Browand FK (1974) Vortex pairing: the mechanism of turbulent mixing-layer growth at moderate Reynolds number. J Fluid Mech 63(2):237-255

[111] Wortmann FX (1969) Visualization of transition. J Fluid Mech 38:473-480

[112] Zaman KBMQ, Hussain AKMF (1980) Vortex pairing in a circular jet under controlled excitation. Part 1. General jet response. J Fluid Mech 101:449-491 


\section{Acknowledgments}

This dissertation is more than a summary of high-tech equipment, numerous data and complicated figures. As I look back through the whole work, I see a logbook which is full of inspiration and practice, discussions and arguments, achievements and frustrations. It is a long journey containing not only my scientific work but also the professional advice, essential support, consistent trust and sincere concern from my professors, colleagues, friends and families, which all the time reminds me of being grateful for everything.

First, I would like to express my sincere gratitude to Dr. Andreas Schröder at the German Aerospace Center (DLR) for his supervision throughout my doctoral study. He always supports me whenever I need in the laboratory, and encourages me for deeper investigations of complicated fluid phenomena. His solid knowledge on fluid mechanics and expertise on measurement technology guided me to convert a new idea to an experiment, and then to a result. This dissertation would not have been accomplished without his guidance.

I sincerely thank Prof. Andreas Dillmann and Prof. Martin Rein at DLR for offering me the opportunity of studying in the GAUSS Program in the University of Göttingen and carrying out my research in the Institute of Aerodynamics and Flow Technology, DLR. Their supervision and trust is of essential importance for this dissertation. I sincerely thank Prof. Eberhard Bodenschatz from the Max Planck Institute for Dynamics and Self-Organization, Prof. Markus Raffel from the University of Hannover, Prof. Andreas Tilgner and Prof. Wolfgang Glatzel from the University of Göttingen for reviewing my dissertation. Their lectures and seminars present various fluid phenomena from a microscopic cell to a giant aircraft, from the atmosphere to a 
star in the universe, showing the intrinsic beauty of the nature.

I gratefully acknowledge the enlightening discussions and continuous concern from Prof. Xiao Ming from Nanjing University of Aeronautics and Astronautics and Dr. Jürgen Kompenhans at DLR. Prof. Ming inspired me a lot in my experimental designs. Dr. Kompenhans and Mrs. Kompenhans warmly hosted my families as guests every Christmas. I also give my sincere appreciation to Prof. Lorenz Sigurdson from University of Alberta, Prof. Christian Kähler from Universität der Bundeswehr München, Prof. Peter Schmidt from Imperial College London, Prof. Nan Jiang from Tianjin University, Prof. Jinjun Wang from Beijing University of Aeronautics and Astronautics, Prof. Haitao Xu from Tsinghua University, Prof. Björn Hof from Institute of Science and Technology Austria, Prof. Hengdong Xi from Northwestern Polytechnical University, Prof. Huixuan Wu from University of Kansas, Dr. Xing Wei from Princeton University, Dr. Duo Xu, Dr. Baofang Song and Dr. Liang Shi from the Max Planck Institute for Dynamics and Self-Organization for inspiring discussions and valuable comments on my dissertation.

I am deeply indebted to my colleagues at DLR, who are experts in the fields of aerodynamics, aeroacoustics, signal processing and the most advanced measurement technology. I thank Dr. Lars Koop, the head of the Department of Experimental Methods, for offering valuable review comments on my publications and financial support for my conference travels. I also thank Dr. Reinhard Geisler and Dr. Daniel Schanz for their close cooperation in wind tunnel tests and generous help in evaluation of tomographic PIV data. My acknowledgment is also given to Dr. Walter Beck, Dr. Christian Klein, Dr. Dirk Michaelis from LaVison, Dr. Thomas Ahlefeldt, Dr. Judith Galuba, Mr. Sebastian Gesemann, Mr. Stefan Haxter and Mr. Björn Wrede, from whom I have learnt a lot. None of my experiments would have been successfully carried out without the technical support from Mr. Janos Agocs, Mr. Hartmut Mattner and Mr. Dirk Otter.

Living in Göttingen is a wonderful experience in my life. In this beautiful town, I have met a lot 
of friends who are not only scientists but also singers, painters, photographers, chefs and football players. They made my stay in Göttingen a joy. I also thank the German Academic Exchange Service (DAAD) for the financial support of my doctoral study in Germany.

I can hardly find the best words to express my most gratitude for my parents. Even though I have accomplished my doctoral study, it started from the day when they taught me to say my first word. Even though I have travelled so far, it started from the moment when they encouraged me to make my first step. I would not have made it without them.

Last but not least, I would like to thank my wife Ting for everything that you have contributed. Your support, understanding and considerate care is an essential contribution to my doctoral title. When finalizing the dissertation, I realize it has been ten years since the day when I first met you in the scenic campus of our university. I am truly grateful beyond words for your affectionate companion every day ever since. To my son Yunxuan, it was the brightest morning in my life when I knew you were coming to us. I am very happy to watch you grow every day and soon become a three-year-old young man with kindness, courage and responsibility.

\section{Xingyu Ma}

Göttingen, May 2015

"Extra Gottingen non est vita, si est vita non est ita." ("Outside of Göttingen there's no life; if there's a life, then none like this.") 


\section{Curriculum Vitae}

\section{Xingyu Ma}

Institute of Aerodynamics and Flow Technology, German Aerospace Center

Bunsenstraße 10, 37073 Göttingen, Germany

\section{Nationality, Date and Place of Birth}

Chinese

27. 09. 1986

Hebei, China

Languages

Chinese (Native)

English (Fluent)

German (Basic)

\section{Education}

$01.2012-09.2015$

Doctoral candidate in Physics, supervisor: Prof. Andreas Dillmann

Department of Physics, Georg-August-University of Göttingen, Germany

$10.2011-09.2015$

DLR-DAAD research fellow,

Institute of Aerodynamics and Flow Technology

German Aerospace Center

$09.2009-06.2011$

Master of Engineering, supervisor: Prof. Xiao Ming

College of Aerospace Engineering 
Nanjing University of Aeronautics and Astronautics, China

$09.2005-06.2009$

Bachelor of Engineering, supervisor: Prof. Xiao Ming

College of Aerospace Engineering

Nanjing University of Aeronautics and Astronautics, China

\section{Publications}

[1] Ma X, Geisler R, Agocs J, Schröder A (2015) Investigation of coherent structures generated by acoustic tube in turbulent flow separation control. Experiments in Fluids 56:46

[2] Ma X, Geisler R, Agocs J, Schröder A (2014) Time-resolved tomographic PIV investigation of turbulent flow control by vortex generators on a backward-facing step. In: Proceedings of the 17th International Symposium on Applications of Laser Techniques to Fluid Mechanics, Lisbon, Portugal

[3] Ma X, Geisler R, Agocs J, Schröder A (2014) Investigation of coherent structures in active flow control over a backward-facing step by PIV. In: Proceedings of the 16th International Symposium on Flow Visualization, Okinawa, Japan

[4] Ma X, Ming X (2012) Experimental research and error analysis on seven-hole probe measurements of shearing flow field. ACTA Aerodynamic Sinica (Chinese Edition) 30(1):57-62

[5] Ma X, Ming X (2011) Characteristics and control of blade tip vortex of wind turbines. Journal of Nanjing University of Aeronautics and Astronautics (Chinese Edition) 43(5):635-639

[6] Bai Y, Ma X, Ming X (2011) Lift enhancement of the airfoil and tip flow control for wind turbine. Applied Mathematics and Mechanics 32(7):774-784

\section{Conference presentations}

[7] Annual workshop on Measurement and Optical Techniques for Aerospace Research (MOTAR), Cologne, Germany, 2015

[8] 17th International Symposium on Applications of Laser Techniques to Fluid Mechanics, Lisbon, Portugal, 2014

[9] 16th International Symposium on Flow Visualization, Okinawa, Japan, 2014

[10] 16th STAB-workshop, Göttingen, Germany, 2013

\section{Awards and Honors}

- DLR-DAAD Research Fellowship, 2011-2015

- Excellent Master Project, 2011

- Excellent Diploma Project, 2009

- National Scholarship of China, 2007

- Shanghai Astronautics Scholarship, 2007

- The First Prize Scholarship of NUAA, 2006, 2007 